Andreas Germershausen, Wilfried Kruse

AUSBILDUNC

STATT AUSQRENZUNG

Wie interkulturelle Öffnung und Diversity-Orientierung in Berlins Öffentlichem Dienst und in Landesbetrieben gelingen können 
Andreas Germershausen, Wilfried Kruse

Ausbildung statt Ausgrenzung

Kultur und soziale Praxis 
Andreas Germershausen, geb. 1952, ist Ethnologe und Sozialwissenschaftler. Er war beim Berliner Senat beschäftigt und unter anderem für die Ausbildung von Migrant"innen zuständig sowie von 2015 bis 2019 Beauftragter für Integration und Migration und Leiter der Integrationsabteilung. An sozialwissenschaftlichen Forschungsinstituten in Bangkok und Berlin war er mit Fragen internationaler Migration und globaler Fluchtbewegungen befasst und arbeitete von 1997 bis 2001 am Asian Research Center for Migration an der Chulalongkorn University in Bangkok.

Wilfried Kruse (Dr.), geb. 1947, Arbeitssoziologe und Berater, ist Koordinator der Arbeitsgemeinschaft »Weinheimer Initiative « und beratend aktiv bei der Öffnung von Berufsausbildung für junge Leute aus Familien mit Einwanderungsgeschichte sowie beim »Bundesverband Netzwerke von Migrantenorganisationen«. Von 1972 bis 2012 war er wissenschaftlicher Mitarbeiter und zeitweilig Geschäftsführender Direktor der Sozialforschungsstelle Dortmund. 
Andreas Germershausen, Wilfried Kruse

\section{Ausbildung statt Ausgrenzung}

Wie interkulturelle Öffnung und Diversity-Orientierung in Berlins

Öffentlichem Dienst und in Landesbetrieben gelingen können 


\section{Bibliografische Information der Deutschen Nationalbibliothek}

Die Deutsche Nationalbibliothek verzeichnet diese Publikation in der Deutschen Nationalbibliografie; detaillierte bibliografische Daten sind im Internet über http://dnb.d-nb.de abrufbar.

\section{(1) (1) ( $\Theta$}

Dieses Werk ist lizenziert unter der Creative Commons Attribution-NonCommercial-NoDerivs 4.0 Lizenz (BY-NC-ND). Diese Lizenz erlaubt die private Nutzung, gestattet aber keine Bearbeitung und keine kommerzielle Nutzung. Weitere Informationen finden Sie unter https://creativecommons.org/licenses/by-nc-nd/4.o/deed.de

Um Genehmigungen für Adaptionen, Übersetzungen, Derivate oder Wiederverwendung zu kommerziellen Zwecken einzuholen, wenden Sie sich bitte an rights@transcript-publishing.com

Die Bedingungen der Creative-Commons-Lizenz gelten nur für Originalmaterial. Die Wiederverwendung von Material aus anderen Quellen (gekennzeichnet mit Quellenangabe) wie z.B. Schaubilder, Abbildungen, Fotos und Textauszüge erfordert ggf. weitere Nutzungsgenehmigungen durch den jeweiligen Rechteinhaber.

\section{(C) 2021 transcript Verlag, Bielefeld}

Umschlaggestaltung: Maria Arndt, Bielefeld

Umschlagabbildung: (c) BQN Berlin, Foto: Mikael Oskarsson

Lektorat: Morten Paul, Berlin

Satz: Mark-Sebastian Schneider, Bielefeld

Druck: Majuskel Medienproduktion $\mathrm{GmbH}$, Wetzlar

Print-ISBN 978-3-8376-5567-4

PDF-ISBN 978-3-8394-5567-8

https://doi.org/10.14361/9783839455678

Gedruckt auf alterungsbeständigem Papier mit chlorfrei gebleichtem Zellstoff. Besuchen Sie uns im Internet: https://www.transcript-verlag.de Unsere aktuelle Vorschau finden Sie unter www.transcript-verlag.de/vorschau-download 


\section{Inhalt}

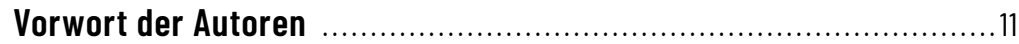

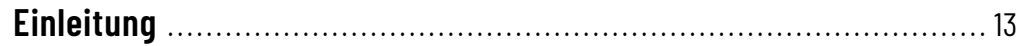

\section{Teil 1 \\ Den Übergang Schule - Arbeitswelt als ein integrationspolitisches Leitthema setzen}

\section{Kapitel 1}

\section{Integrationspolitik wird konkret:}

Berufsausbildung als ein wichtiges Handlungsfeld

19

Ende der 1990er Jahre: Deutschland auf dem Weg

zu einer erneuerten Integrationspolitik ........................................... 21

Migrant*innen: Von der Anwesenheit zur Zugehörigkeit? ........................22

Vielfalt fördern - Zusammenhalt stärken: Acht Handlungsstrategien ........... 23

Öffnung der Berufsausbildung als wichtiges Handlungsfeld ...................... 24

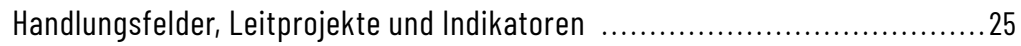

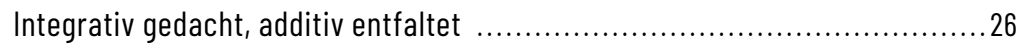

Übergang Schule - Arbeitswelt integrationspolitisch schon vor 2007 Thema ... 27

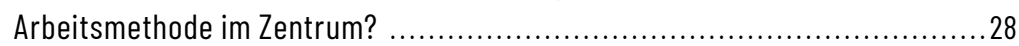

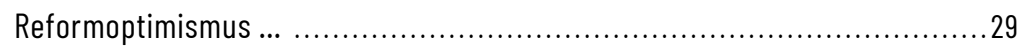

Integration und Desintegration im Wettlauf? ......................................... 30

Idee: Integrationspolitischer Qualitätstreiber

für den Übergang Schule - Beruf .................................................. 30

Leitprojekt »Erhöhung der Ausbildung im Öffentlichen Dienst « ... ............... 31

... und seine Umsetzung als Kampagne Berlin braucht dich! ........................32

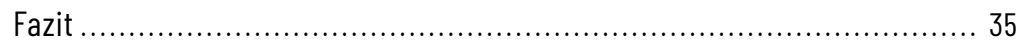




\section{Kapitel 2}

Was ein Integrationsbeauftragter überhaupt bewegen kann .......... 39

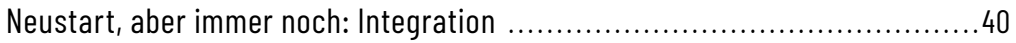

Integrationsbeauftragter: Institutionelle Einbindung ............................ 41

Integration und Teilhabe als Querschnittsaufgabe .................................... 44

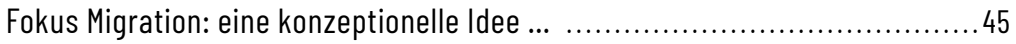

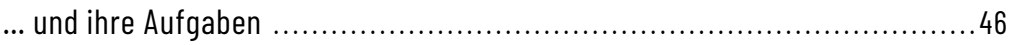

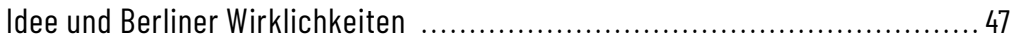

Verknüpfungschancen zwischen verschiedenen Politikfeldern

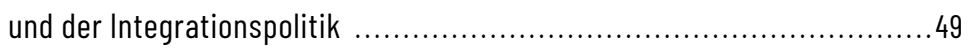

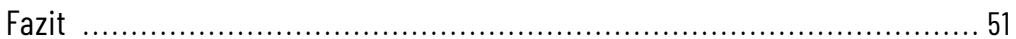

\section{Kapitel 3}

\section{Die Ausgangslage beim Übergang Schule -}

Arbeitswelt in Berlin in den 2010er Jahren ....................................5 55

Der Übergang von der Schule in die Arbeitswelt wird langsam zum Thema .....56

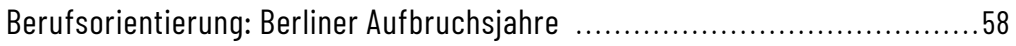

Ideen zu einem Übergangsmanagement - und was daraus wird ..................60

Ein Entwicklungsprojekt: das Landeskonzept Berufs-

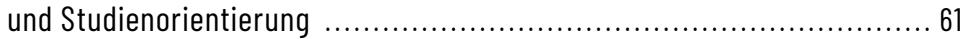

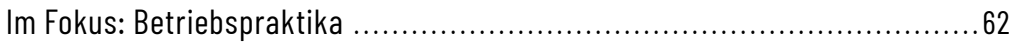

Mehr Aufmerksamkeit für die beruflichen Perspektiven

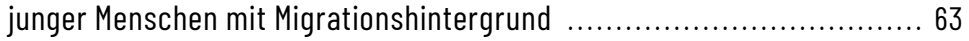

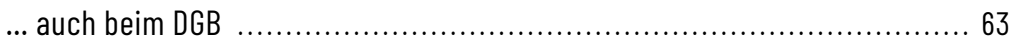

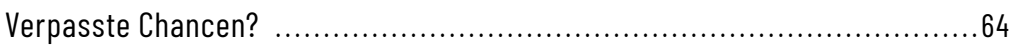

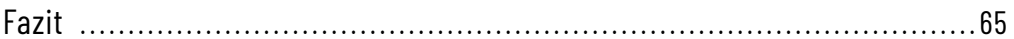

\section{Kapitel 4}

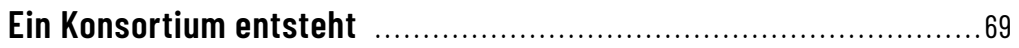

Die Anfänge: Eine Kampagne öffentlicher Arbeitgeber ............................69

Bereitstellung von Betriebsbegegnungen:

Initial für die Entstehung des Konsortiums ..................................... 70

Die etwas andere Konsortial-Idee: Betriebe und Schulen gemeinsam ............ 71

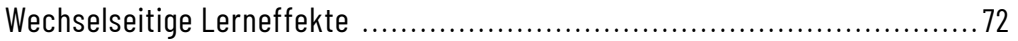

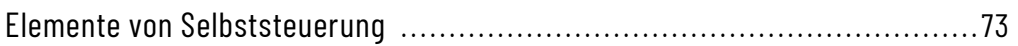

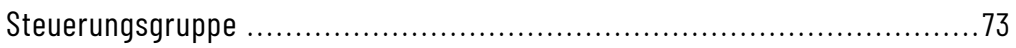

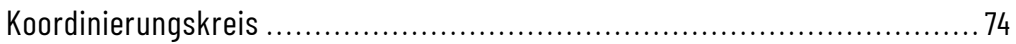




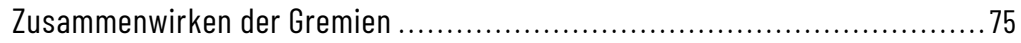

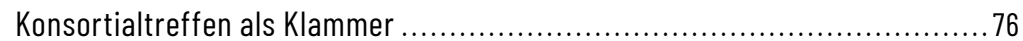

Koordinierung: Mehr Routine als Impulse ............................................. 76

Zugehörigkeit: Eine Bindung mit Langzeitwirkung ................................. 77

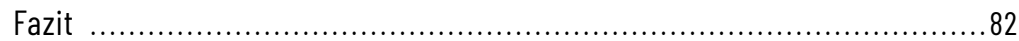

\section{Kapitel 5}

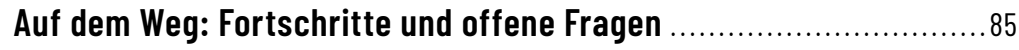

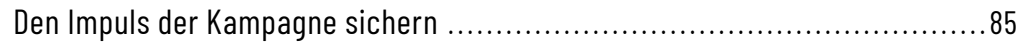

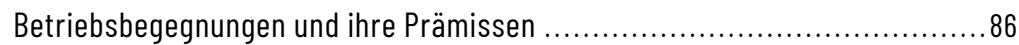

Kooperation mit Schulen: Welche Schulen? ........................................ 88

Erste Kooperationserfahrungen und die Erfindung des »matching-too|« ......88 88

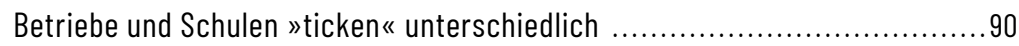

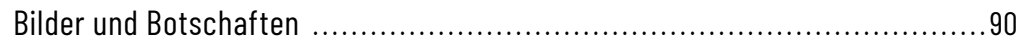

Schwierig: Ein realistischer Blick auf die Jugendlichen ........................... 91

Beim Start: Fehlende oder schwache Einbettung

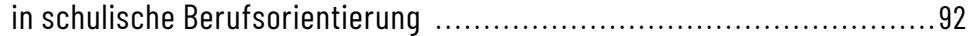

Qualifizierte Vierstufigkeit: gut gedacht, schwer umzusetzen ................. 93

Berlin braucht dich!-Plätze: immer kontingentiert ................................ 94

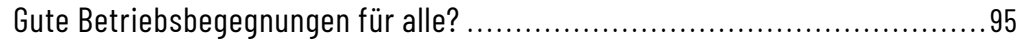

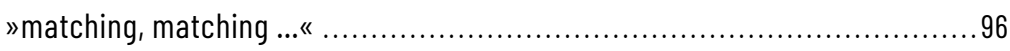

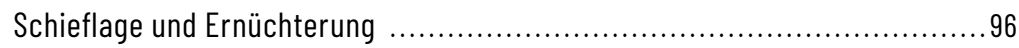

Weiter mit denselben Partnern? ..................................................... 97

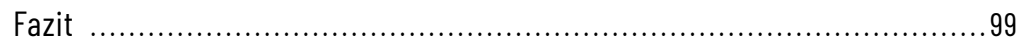

\section{Teil 2}

Ausbildung tatsächlich für Vielfalt öffnen

\section{Kapitel 6}

\section{Hartnäckige Benachteiligungen und die Bedeutung}

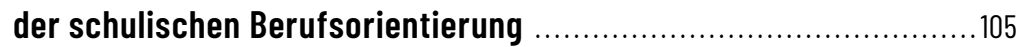

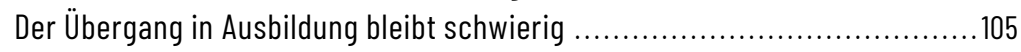

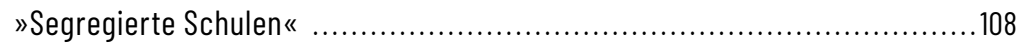

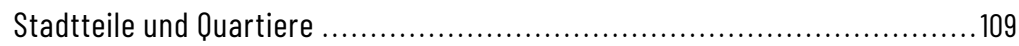

Die Schulen stärken - aber wie? ....................................................111

Übergangsverläufe: Differenzierungen innerhalb der Schüler*innenschaft ... 112 
Über die Entstehung prekärer Übergangsverläufe …............................ 114

Weichenstellungen für prekäre Übergangsverläufe ............................. 117

Praktisch-betriebliche Berufsorientierung:

ein Fenster zum Leben außerhalb der Schule ................................ 119

Zur »Kunst» der Einbettung von Praktika in schulische Berufsorientierung ...120

Fazit

\section{Kapitel 7}

Kann beruflich-betriebliche Bildung

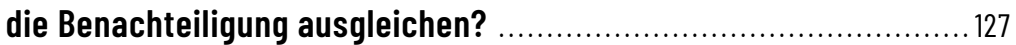

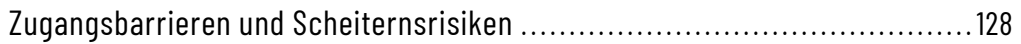

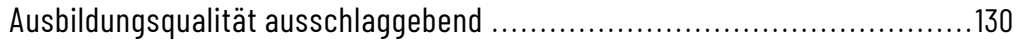

Den Blick auch auf den zweiten zentralen Lernort richten:

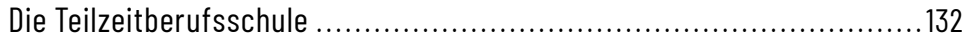

Der pädagogische Beitrag der Berufsschulen .................................... 133

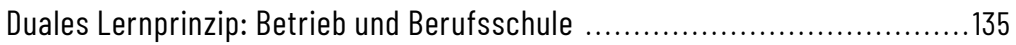

Pädagogische Lernortkooperation ist integrationspolitisch

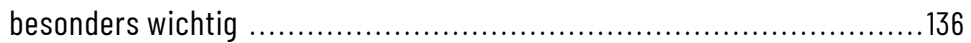

Das Teilhabe-Potenzial der Berufsausbildung ausbauen und nutzen ............138

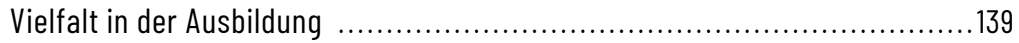

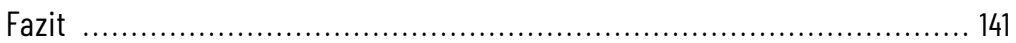

\section{Kapitel 8}

Ausbildung für Vielfalt öffnen: Ein neuer Anlauf ........................... 145

Konsortialtagung 2015: Den Betrieb als Lernort

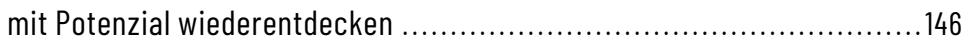

Die Auswahlkriterien für Ausbildungsplätze werden hinterfragt ................ 147

Differenzierte Sicht auf die Schüler*innen, um die es geht .......................148

Grundzüge eines »Pilotvorhabens« zu neuen Wegen

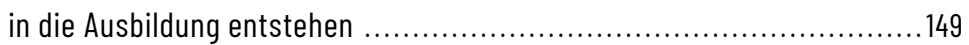

Schulen und Betriebe wechselseitig aufeinander angewiesen ................... 150

Das "Setting« von Berlin braucht dich! muss angepasst werden ................ 151

Ausbildung: eine Frage der Einstellung? Erstmal erproben ... ....................153

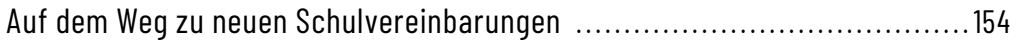

Benachteiligung abbauen: Nur mit einem breiten Netz

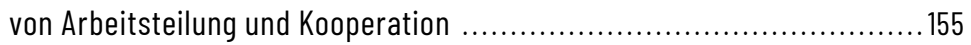

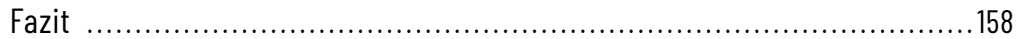




\section{Kapitel 9}

Nach dem "Langen Sommer des Willkommens" ............................ 161

Auf die vielen Geflüchteten wenig vorbereitet ..................................... 162

Schritte und Bausteine für Integration und Teilhabe ….......................... 164

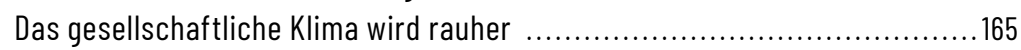

Das Schulsystem bleibt eine "große Baustelle « ...................................166

Die Herausforderung »Geflüchtete«: ein Schub für die Integrationspolitik? ... 167

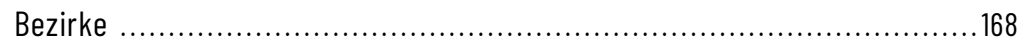

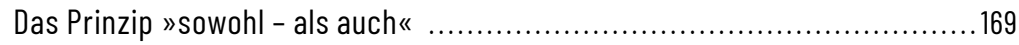

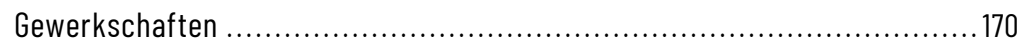

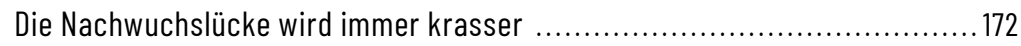

»Wir sind in der Realität schon weiter « ....................................................... 173

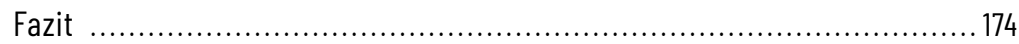

\section{Kapitel 10}

Ausblick: Übergänge insgesamt in den Blick nehmen ................. 177

Blick auf duale Ausbildung integrationspolitisch wichtig, aber zu eng .......... 178

Wie attraktiv ist Berufsausbildung für Jugendliche? ............................. 179

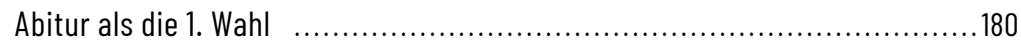

Ein doppelter Ansatz: Betriebe müssen sich für Vielfalt öffnen .................. 181

Ein erweitertes Verständnis vom Übergang Schule - Arbeitswelt ................ 182

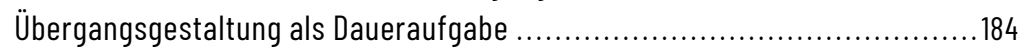

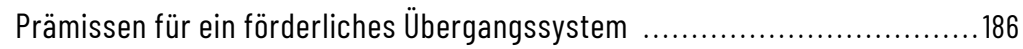

DGB: Gelingende Übergänge von der Schule in die Arbeitswelt ................... 189

Übergänge: ein Lehrstück ......................................................... 190

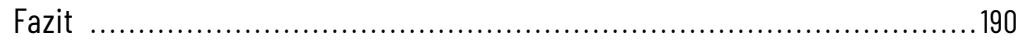

\section{Nachbetrachtung}

Andreas Germershausen im Gespräch mit Wilfried Kruse ...............193

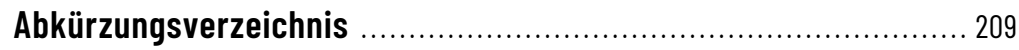

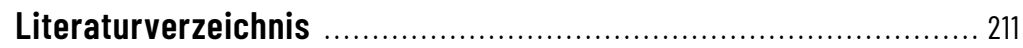





\section{Vorwort der Autoren}

Das vorliegende Buch zeichnet mehr als ein Jahrzehnt Berliner Teilhabepolitik - bislang auch als Integrationspolitik bezeichnet - nach, und zwar in dem Feld der Öffnung von Berufsausbildung für junge Menschen aus Familien mit Einwanderungsgeschichte. Es nimmt zwar Bezug auf das Leitprojekt Berlin braucht dich!, stellt aber den komplexen Prozess des »Öffnens« von Berufsausbildung insgesamt dar und benennt Chancen, aber auch Schwierigkeiten dieses Prozesses. Es resümiert und zeigt gleichzeitig die Potenziale für eine Weiterführung der Teilhabepolitik in diesem Feld auf.

Im medial mitgeprägten Politikgeschäft wird schnell etwas als nicht mehr aktuell »zu den Akten gelegt«. Die in diesem Buch beschriebene Teilhabepolitik hat aber Aktualität behalten und möglicherweise sogar hinzugewonnen. Aktuell bleibt sie, weil die gesellschaftliche Herausforderung, die 2006 mit dem Ziel der Öffnung von Berufsausbildung für Jugendliche aus Familien mit Einwanderungsgeschichte adressiert war, weiterhin besteht. Trotz erheblich gestiegener Zahlen bleibt der Anteil dieser Jugendlichen an den Ausbildungsverhältnissen noch deutlich hinter dem in der Bevölkerung zurück. Dies gilt in besonderer Weise für den klassischen Öffentlichen Dienst.

Ebenso problematisch, wenn nicht noch bedenklicher ist der Umstand, dass Jugendliche aus Familien mit Einwanderungsgeschichte, die sich in mehrfachen Risikolagen, wie z.B. Armut und Distanz zur Schule, befinden, auch bei guter Motivation wenig Zugang zu einer Berufsausbildung erhalten. Im Buch werden die Bemühungen und systematischen Ansätze nachgezeichnet und analysiert, hierin zu durchgreifenden Verbesserungen zu kommen. Es gab und gibt Erfolge, aber noch nicht in der erforderlichen Breite.

Die gesellschaftliche Gefahr, dass ein nicht kleiner Teil der nachwachsenden Generation in Berlin randständig bleibt oder wird, ist also noch kei- 
neswegs behoben. Diese Gefahr könnte sogar wachsen, denn die Proteste, die sich im Sommer 2020 mit dem Slogan Black Lives Matter verbunden haben, weisen auf vielfältige Zusammenhänge zwischen sozialer Lage, Lebenschancen und Diskriminierung hin. Und es steht zu befürchten, dass die sozialen Folgen der Corona-Pandemie ungleich wirken, oder genauer: die soziale Ungleichheit verstärken.

Eine konzentrierte und koordinierte Teilhabepolitik - zentrales Thema des Buches - bleibt dringlich. Hierzu soll im Übrigen auch die aktuelle Novelle des zu seiner Zeit zukunftweisenden Berliner Partizipations- und Integrationsgesetzes aus dem Jahr 2010 beitragen, dessen Titel»Gesetz zur Förderung der Partizipation in der Migrationsgesellschaft « nun nachvollzieht, dass Berlin schon seit langer Zeit unbestreitbar eine Einwanderungsmetropole ist.

Wir danken dem BQN-Team für seine Arbeit, Klaus Kohlmeyer für vielfältige Zeitzeugenschaft, Ratschläge und Unterstützung, Ulrich Raiser für eine kritisch-freundschaftliche Lektüre, Morten Paul für die zugewandte und genaue Lektorierung des Textes.

Berlin, im Januar 2021

Andreas Germershausen

Wilfried Kruse 


\section{Einleitung}

In den Jahren 2006-2008 wird die interkulturelle Öffnung von Berufsausbildung zu einem festen und zunehmend auch charakteristischen Bestandteil der vom Beauftragten des Berliner Senats für Integration und Migration betriebenen Initiativen. Dies baute u.a. auf Erfahrungen und Einsichten auf, die aus der Beteiligung am Bundesprogramm zu »Lokalen Netzwerken für die berufliche Qualifizierung junger Migrantinnen und Migranten« gewonnen worden waren.

Den Rahmen hierfür bildete das 2007 vorgelegte Integrationskonzept "Vielfalt fördern - Zusammenhalt stärken«. Dort wurde u.a. als strategisches Leitprojekt die Öffnung der Berufsausbildung im Öffentlichen Dienst definiert. Einen entscheidenden Impuls erhielt die Umsetzung dieses Leitprojekts durch die senatsseitige Bereitschaft, den Öffentlichen Dienst und die Landesbetriebe unter dem Motto »Berlin braucht dich!« für junge Leute mit Migrationsgeschichte zu öffnen. Was zunächst als Kampagne startete, wurde dann in der Folge systematisiert und vorsichtig institutionalisiert sowie durch diverse andere Maßnahmen flankiert, ergänzt und erweitert.

Zehn Jahre später haben sich die Rahmenbedingungen für die (weitere) interkulturelle Öffnung von Berufsausbildung verändert, ohne dass sie ihre Bedeutung als wichtiges integrationspolitisches Handlungsfeld verloren hat. Die Vorzeichen auf dem Arbeitsmarkt haben sich so verändert, dass Fachkräftemangel zu einem wichtigen Thema geworden ist. Die Berufsorientierung an den Sekundarschulen hat sich wesentlich verbessert und bietet im erheblichen Umfang Betriebsbegegnungen als Erfahrungsfeld, was durchaus auch auf Impulse aus Berlin braucht dich! zurückzuführen ist.

Gleichberechtigte Teilhabe der Menschen mit Migrationsgeschichte ist als politisches Ziel in Berlin breit akzeptiert. Auf dem engeren Feld der interkulturellen Öffnung von Berufsausbildung ist ein erheblicher Anstieg des 
Anteils von jungen Leuten aus Familien mit Einwanderungsgeschichte zu beobachten - und zwar nicht nur in den Betrieben mit Landesbeteiligung, sondern z.B. auch im Sektor von Metall \& Elektro, der bei Berlin braucht dich! mitarbeitet, und in anderen Wirtschaftsbereichen. Diese insgesamt positive Bewertung gilt trotz der Tatsache, dass sich in der letzten Zeit ausländerfeindliche und rassistische Ressentiments verstärkt haben. Auch die Ankunft einer großen Zahl von Geflüchteten in den Jahren 2014 bis Anfang 2016 hat die Trends zur interkulturellen Öffnung der Berufsausbildung nicht eingeschränkt.

Überall ist jedoch noch - auch vor dem Hintergrund des absehbaren Fachkräftemangels und sozialer Spaltungsrisiken - »Luft nach oben«. Vor allem aber ist alarmierend, dass Jugendlichen aus Sekundarschulen mit sehr hohen Anteilen an Schüler*innen aus Familien mit Migrationsgeschichte und zugleich SGB II-Bezug nach wie vor nur im geringen Maß der (direkte) Übergang in eine Berufsausbildung gelingt. Es bedarf also offenkundig erheblicher weiterer - und auch auf pädagogisches Handeln fokussierter Anstrengungen, damit Betriebe und Berufsschulen ohne Scheu und erfolgreich mit Vielfalt umgehen können. Diese Herausforderung stand auch im Zentrum des gemeinsam zwischen dem Integrationsbeauftragten und der Arbeitsgemeinschaft Weinheimer Initiative im Dezember 2015 durchgeführten Forums zum Thema »Vielfalt in der Ausbildung«.

Erhebliche Fortschritte und fortbestehende Hemmnisse zeichnen also nach zehn Jahren ein komplexes Bild im Handlungsfeld »Interkulturelle Öffnung von Berufsausbildung « in Berlin. Besorgniserregend ist die Tatsache, dass die Benachteiligung bestimmter Gruppen von Jugendlichen auf ihrem Weg ins Berufsleben viel hartnäckiger ist als erwartet, wenngleich sich die Rahmenbedingungen im Zeitraum verbessert haben.

Die Aktivitäten, die vom Integrationsbeauftragten ausgingen - insbesondere jene von Berlin braucht dich! - kann man deshalb auch als eine Sonde in eine schwierige gesellschaftliche Realität verstehen, wenn man so will, also als ein »lernendes Projekt «.

Andreas Germershausen, Integrationsbeauftragter bis März 2019, verantwortete dieses Handlungsfeld im Arbeitsbereich des Beauftragten für Integration und Migration von Anfang an. Eine Zwischensichtung und der Versuch, auf deren Basis einige Hinweise für die Weiterarbeit zu geben, bieten sich also nach diesen zehn Jahren an. Als einer der Ko-Autoren wird diese 
Zwischensichtung stark durch seine Erfahrungen und Einschätzungen mitgeprägt. Der Zweite im Autoren-Team ist Dr. Wilfried Kruse, praktisch seit der Startzeit 2006 wissenschaftlicher Berater von Berlin braucht dich! und immer wieder auch direkt des Integrationsbeauftragten. Im Unterschied zu einer wissenschaftlichen Begleitung oder Evaluation zielt die nahe am Prozess angesiedelte Beratung auf die Anregung und Unterstützung einer kontinuierlichen Reflexion des Fortgangs der Arbeiten, ihrer Zielorientierung und den Chancen und Schwierigkeiten der Zielerreichung.

Das Autoren-Team bietet also eine Kombination aus fachlich-politischer Verantwortung und Begleitung als »kritischer Freund«, von Nähe und Distanz, von Beobachtung, Beteiligung und Analyse: eine Kombination, die für komplexe Projekte, von denen dieses Buch handelt, hoffentlich produktiv ist.

Die Kapitel wurden auf der Basis unterschiedlicher Materialien und Methoden erarbeitet. Zugrunde gelegt sind die vorhandenen offiziellen Dokumente und veröffentlichte Studien sowie auch die Expertisen, die im Zusammenhang mit der Integrationspolitik im Feld der »interkulturellen Öffnung der Berufsausbildung« in Berlin entstanden sind.

Der Aufbau des Textes folgt der Überzeugung, dass das im Zentrum stehende integrationspolitische Handeln seine Voraussetzungen und Wirkungen in der gesellschaftlichen Wirklichkeit reflektiert und es daraufhin ggf. korrigiert oder modifiziert werden, sich also lernfähig halten muss. Entstanden ist eine Art von Fallstudie zu Integrationspolitik im Feld des Übergangs von der Schule in die Arbeitswelt, die sich nicht chronologisch, sondern von einem in den Jahren 2015/2016 deutlich werdenden kritischen Zwischenbefund her erschließt. Es zeigte sich nämlich, was die Anteile von Auszubildenden aus Familien mit Migrationsgeschichte betraf, ein gespaltenes Ergebnis: In den an Berlin braucht dich! beteiligten Betrieben und Verwaltungen war deren Anteil an der Ausbildung kontinuierlich und deutlich gestiegen, während zugleich in den an Berlin braucht dich! ebenfalls beteiligten Integrierten Sekundarschulen die Übergänge in Ausbildung verschwindend gering geblieben waren. Dies war schockierend und irritierend zugleich und musste zu einer Überprüfung der bisherigen Ansätze und Arrangements führen, die schließlich in einer Re-Orientierung mündeten.

Um diesen Einschnitt deutlich zu machen, umfasst der Text zwei große Teile: Teil 1 führt auf diesen Punkt hin, Teil 2 nimmt von ihm seinen Ausgang. Teil 1 hat die Überschrift »Den Übergang Schule - Arbeitswelt als ein 
integrationspolitisches Leitthema setzen", der zweite Teil die Überschrift: "Ausbildung tatsächlich für Vielfalt öffnen«.

Insgesamt nimmt der Text einen Zeitraum von 2006 bis 2018 in den Blick, also mehr als zehn Jahre. Im ersten Teil führen die Kapitel 1 und 2 in die zu Beginn dieses Zeitraums neu aufgestellte Integrationspolitik des Landes Berlin ein, wobei dies immer wieder in Bezug auf das Handlungsfeld Berufsausbildung, bzw. Übergang von der Schule in die Arbeitswelt, konkretisiert wird. Die Kapitel 3 und 4 diskutieren das Konzept des integrationspolitischen Leitprojekts Berlin braucht dich!, seine Umsetzung und seine integrationspolitische Einbettung. Dies ist zugleich die Grundlage für die Beschreibung der Ansätze zur Re-Orientierung, die sich in Kapitel 8 finden. Der Teil 2 beginnt aber mit einer durch den kritischen Zwischenbefund notwendig gewordenen vertieften Vergewisserung über die Gründe für die hartnäckigen Benachteiligungen bestimmter Gruppen von jungen Menschen aus Familien mit Migrationsgeschichte und über die Teilhabe-Chancen, die eine Berufsausbildung von guter Qualität ihnen bieten könnte (Kapitel 6 und 7). Die dem Kapitel 8 dann nachfolgenden Kapitel öffnen den Blick erneut über Berlin braucht dich! hinaus zunächst auf die vergangenen Jahre, die durch eine erhebliche Zahl neu angekommener junger Geflüchteter mitgeprägt war (Kapitel 9), um dann Umrisse eines Übergangssystems zu beschreiben, das dem Anspruch, benachteiligungs- und interkulturell sensibel zu sein, näherkommt (Kapitel 10).

Mit diesem Kapitel schließt sich auch der Bogen zum 1. Kapitel - ein Bogen, der zehn Jahre integrationspolitischer Entwicklung in Berlin vor allem im Feld von Übergang Schule - Arbeitswelt und Berufsausbildung abschreitet. Innerhalb dieses Bogens kann mit den Kapiteln 3 bis 5, teilweise 6 und 7, vor allem aber auch 8 die Entwicklung des integrationspolitischen Leitvorhabens Berlin braucht dich! als Beispiel für Integrationspolitik in diesem Feld nachvollzogen werden, und eben nicht nur im Sinne von Umsetzung, sondern auch im Sinne von Impulsen für eine Integrationspolitik, die sich für Weiterentwicklungen offenhält.

Die Kapitel 1 bis 10 verantworten die beiden Autoren gemeinsam; es folgt eine Schlussbetrachtung in Gesprächsform, in der Andreas Germershausen auch eine Art persönlicher Bilanz vornimmt. 
Teil 1

Den Übergang Schule - Arbeitswelt als ein integrationspolitisches

Leitthema setzen 



\section{Kapitel 1}

\section{Integrationspolitik wird konkret: Berufsausbildung als ein wichtiges Handlungsfeld}

2007 beginnt mit der Vorlage des Berliner Integrationskonzepts »Vielfalt fördern - Zusammenhalt stärken« eine neue Etappe der Berliner Integrationspolitik. ${ }^{1}$ Dieses Konzept baut auf seinem Vorläufer, dem ersten Berliner Integrationskonzept mit gleichem Titel von 2005, auf, trägt aber vor allem der auf dessen Grundlage erfolgten breiten fachlich-politischen Debatte Rechnung.

Der eher pessimistische Grundduktus des Konzepts von 2005 war noch durch das bundespolitische »Erbe« der verspäteten Anerkennung von Deutschland als Einwanderungsland geprägt. Zudem hatte der massive DeIndustrialisierungsprozess nach 1989 - insbesondere in Berlin - Menschen mit Migrationsgeschichte besonders stark negativ betroffen. Das Konzept von 2007 sieht trotz anhaltend schwieriger Rahmenbedingungen dagegen einige Verbesserungstendenzen bei Bildung und Arbeitsmarkt, an die positiv angeknüpft werden könne.

Schon 2005 war festgehalten worden: »Integrationspolitik ist eine ressortübergreifende Querschnittsaufgabe, die einer stärkeren strategischen Steuerung und eines Integrationsmonitorings bedarf. $\ll^{2}$ Das 2007 vorgelegte Integrationskonzept folgte dann der Aufforderung des Abgeordnetenhau-

1 Der Beauftragte des Senats von Berlin für Integration und Migration (Hg.): Vielfalt fördern-Zusammenhalt stärken. Das Berliner Integrationskonzept, Berlin 2007, Drucksache 16/0715. Der Senat hat das Integrationskonzept am 3.7.2007 beschlossen.

2 Ebd.S. 3. 
ses, ein "mittelfristiges Arbeitsprogramm zur Integrationssteuerung zu erarbeiten, in dem konkrete Ziele, Maßnahmen und Berichterstattungsverfahren sowie zur Überprüfung der Arbeitsergebnisse messbare Indikatoren festgelegt werden. ${ }^{3}$ Das Konzept umfasst acht Handlungsstrategien und ein Integrationsmonitoring als Steuerungsgrundlage. ${ }^{4}$ Zuvor waren 2006 mit der Einrichtung einer Senatsverwaltung für Arbeit, Integration und Soziales auch Zuständigkeiten entschieden worden. 2010 erfolgte in Berlin als erstem Bundesland zudem die Verabschiedung eines Partizipations- und Integrationsgesetzes, ${ }^{5}$ das auch eine gesetzliche Grundlage für den 2003 eingerichteten Landesbeirat für Integrations- und Migrationsfragen (Landesbeirat) schuf. ${ }^{6}$

Warum markiert die Vorlage des Integrationskonzepts von 2007 den Beginn einer neuen Etappe in der Berliner Integrationspolitik? Es handelt sich um die definitive Abkehr von der Tradition einer "Ausländerpolitik«, die in ihrer positiven, in Berlin vertretenen Variante vor allem auf eine Überwindung von Fremdheit durch Information und Begegnung, Respekt und die Förderung friedvollen Zusammenlebens ausgerichtet war. Das Integrationskonzept von 2005 geht bereits von einem Verständnis von Berlin als Einwanderungsstadt aus, das die hier zum Teil schon seit Jahrzehnten lebenden Menschen mit Migrationsgeschichte als dauerhaften Teil einer vielfältigen Einwohnerschaft, nicht als »Problem« und »Last«, sondern als »Normalität« und »Potenzial« sieht. Das Integrationskonzept von 2007 geht aber wichtige Schritte weiter, weil es dieses Verständnis strategisch und operationell wendet. Damit ist Berlin sowohl als Bundesland als auch als Stadt Pionier.

3 Ebd. S. 5.

4 Die Entwicklung der Indikatoren konnte auch auf die Ergebnisse des transnationalen Projekts »Indikatoren für die Zuwandererintegration«zurückgreifen, das im Rahmen des EUProgramms INTI gefördert wurde. VgI. Der Beauftragte des Senats von Berlin für Integration und Migration: Indikatoren zur Messung von Integrationserfolgen (=Berliner Beiträge zur Integration und Migration), Berlin 2007.

5 Weiss, Karin/Alan, Birsan: »Integrationsgesetze in den Bundesländern«, in: Karl-Heinz Meier-Braun,/Reinhold Weber (Hg.), Deutschland Einwanderungsland. Begriffe - Fakten - Kontroversen, Stuttgart 2017, S. 270-273.

6 Zu dessen Vorgeschichte vgl. u.a. Çinar, Safter: »Das Partizipations- und Integrationsgesetz für Berlin«, https://heimatkunde.boell.de/de/2010/10/01/das-partizipations-und-integrationsgesetz-fuer-berlin vom 1.10.2010. 


\section{Ende der 1990er Jahre: Deutschland auf dem Weg zu einer erneuerten Integrationspolitik}

Seit Ende der 1990er Jahre sind in der staatlichen Zuwanderungs- und Integrationspolitik der Bundesrepublik zahlreiche Änderungen und Neuerungen erfolgt. »Von der Zielsetzung her sind diese Maßnahmen vor allem darauf gerichtet, Defizite und Widersprüche der überkommenen Zuwanderungsund Integrationspolitik abzubauen und auf neue Herausforderungen in diesem Politikfeld angemessen zu reagieren. « Die Einschätzungen darüber, wie weitgehend diese Veränderungen sind, gingen damals auseinander. Nach Einschätzung des Sachverständigenrats deutscher Stiftungen für Integration und Migration von 2010 (SVR) jedenfalls sei »in den letzten zehn Jahren in Sachen Integrationspolitik mehr geschehen als in den vier Jahrzehnten zuvor.$^{8}$ Immerhin war eine erhebliche Zeit vergangen, seit im Jahr 1979 von Heinz Kühn, dem ersten »Bundesbeauftragten für die Integration der ausländischen Arbeitnehmer und ihrer Familien« das Memorandum "Stand und Weiterentwicklung der Integration der ausländischen Arbeitnehmer und ihrer Familien in der Bundesrepublik Deutschland« vorgelegt worden war. Schon dessen zentrale Aussage war dabei, dass Deutschland faktisch ein Einwanderungsland sei.

$\mathrm{Zu}$ den wichtigsten Elementen der erneuerten Integrationspolitik zählen die im Jahr 2000 in Kraft getretene Reform des Staatsangehörigkeitsrechts, die 2005 und 2007 erfolgten umfassenden Novellierungen des Ausländerrechts durch das Zuwanderungs- und Aufenthaltsgesetz, das im Sommer 2006 in Kraft getretene Allgemeine Gleichbehandlungsgesetz, die Integrationsgipfel, zu denen Bundeskanzlerin Merkel einlud, die Einrichtung der Deutschen Islamkonferenz auf Bundesebene, Änderungen von Zuständigkeiten auf Bundes-, Landes- und kommunaler Ebene und die Entwicklung, Umsetzung sowie Fortschreibung von Integrationsplänen auf diesen Ebenen. ${ }^{9}$

7 Schulte, Axel: »Integrationspolitik in der Bundesrepublik Deutschland«, in: Der moderne Staat-Zeitschrift für Public Policy 1/2011, S. 29-60, hier S. 38. Im Folgenden wird weiter auf diesen Aufsatz Bezug genommen.

8 SVR: Einwanderungsgesellschaft 2010. Jahresgutachten 2010 mit Integrationsbarometer, Berlin 2010, S. 20.

9 Ein Beispiel hierfür: Kruse, Wilfried/Mütherich, Birgit: Arbeitsmarktintegration von Migrantinnen und Migranten in Dortmund - Eine Handlungshilfe (=sfs Beiträge aus der Forschung), Dortmund 2000. 
Im Sommer 2000 berief Bundesinnenminister Otto Schily eine »Unabhängige Kommission `Zuwanderung «. Sie erarbeitete unter dem Vorsitz der CDUPolitikerin und ehemaligen Bundestagspräsidentin Rita Süssmuth einen ausführlichen Bericht, der im Juli 2001 vorgelegt wurde und das Zuwanderungsgesetz von 2005 vorbereitete. Im Bericht der Süssmuth-Kommission wurden auch Vorschläge der Steuerung von Zuwanderung in Anlehnung an das damalige kanadische Modell gemacht. Insgesamt kann gesagt werden:

Die neuere Integrationspolitik enthält Elemente, die darauf hinweisen, dass die Entwicklung Deutschlands zu einer Einwanderungsgesellschaft und somit nicht nur die Anwesenheit, sondern auch die Zugehörigkeit der Immigranten zu dieser Gesellschaft inzwischen grundsätzlich, wenn auch nicht ohne Vorbehalte, akzeptiert und als Herausforderung für eine gesellschaftliche Cestaltung verstanden werden. So wurde die Förderung von Integration erstmals als öffentliche Aufgabe auf der gesetzlichen Ebene des Aufenthaltsrechts verankert. ${ }^{10}$

\section{Migrant*innen: Von der Anwesenheit zur Zugehörigkeit?}

Von der Anwesenheit zur Zugehörigkeit: Das beschreibt den in diesen Jahren allmählich erfolgenden Perspektivwechsel gut. Das Berliner Integrationskonzept steht hierfür. Zur Beschreibung der Entwicklung des Umgangs mit Einwanderung in Deutschland werden verschiedene Periodisierungen vorgeschlagen, die sich für die Zeit vor 1989 in der Regel ausschließlich auf die BRD beziehen. Unterschieden wird dabei z.B. eine Gastarbeiterphase (19551973), erste Integrationsversuche (1973-1981), eine Abwehrphase (1981-1998) und eine Akzeptanzphase (seit 1998). ${ }^{11}$

Eine andere, problemorientiertere Periodisierung liest sich so: Die Nachkriegszeit und die 1950er Jahre: Flüchtlinge und Vertriebene; die 1950er und 1960er: >Gastarbeiter und die Idee der Rotation; die 1970er: Der Anwerbestopp 1973 und seine Effekte; die 1980er: >Deutschland ist kein Einwanderungsland - zwischen >defensivem Dementi< und pragmatischer Routine; die 1990er:

\footnotetext{
10 A. Schulte: »Integrationspolitik in der Bundesrepublik Deutschland«, S. 41.

11 Geißler, Rainer: »Migration und Integration«, www.bpb.de/izpb/198020/migration-undintegration vom 16.12.2014.
} 
Flüchtlinge, Aussiedler*innen, Fremdenfeindlichkeit; die 2000er: PolicyChange und das Ende des Nicht-Einwanderungslands. ${ }^{12}$ Die DDR und die dortige Aufnahme von politischen Flüchtlingen und Vertragsarbeiter*innen werden auch hier ausgespart.

Gemeinsam ist beiden Periodisierungen, dass sie auf einen langen Zeitraum, nämlich ab Beginn der 1980er Jahre, hinweisen, in dem mit der faktisch vorhandenen und wachsenden Einwanderung abwehrend umgegangen wurde. Diese Abwehr verschärfte sich noch einmal Ende der 1980er und Anfang der 1990er Jahre und kulminierte in den ausländerfeindlichen Ereignissen nach 1989. Sie wurden zugleich zum Ausgangspunkt einer Veränderung des Umgangs mit Einwanderung. Diese Abwehrphase und ihre - wenn auch nur schrittweise erfolgende - Überwindung sind die weitere Vorgeschichte der Berliner Integrationspolitik der $2000 e r$ Jahre.

\section{Vielfalt fördern - Zusammenhalt stärken: Acht Handlungsstrategien}

Schon die acht Handlungsstrategien, die das Integrationskonzept von 2007 aufruft, weisen die neue Integrationspolitik nicht als Ergänzungs- und Sonder-, sondern als Querschnittspolitik aus - jedenfalls dem Anspruch nach. Sie lauten:

1. eine Stadt, viele Lebenswelten: Erfolgreiche Integrationspolitik nutzt die Stärken der europäischen Metropole mit internationaler Anziehungskraft;

2. eine berufliche Perspektive für jede/n: Erfolgreiche Integrationspolitik schafft Zugang zu Ausbildung, Arbeit und Erwerb;

3. Talente erkennen und fördern: Erfolgreiche Integrationspolitik definiert den Bildungsauftrag von Kita und Schule neu;

4. für alle Bürger*innen da: Erfolgreiche Integrationspolitik schafft eine neue Kundenorientierung in der Berliner Verwaltung und den sozialen Diensten;

5. Ausgleich und Verantwortung: Erfolgreiche Integrationspolitik stärkt den Zusammenhalt im Stadtraum;

12 Hoesch, Kirsten: Migration und Integration. Eine Einführung, Wiesbaden 2018. 
6. Aktivierung und Teilhabe: Erfolgreiche Integrationspolitik bekämpft Diskriminierung, ist partizipativ und stärkt die Zivilgesellschaft;

7. gegen Ausgrenzung: Erfolgreiche Integrationspolitik gibt Flüchtlingen eine Perspektive;

8. gemeinsame Ziele, geteilte Verantwortung: Erfolgreiche Integrationspolitik stärkt die Kooperation zwischen Senat und Bezirken. ${ }^{13}$

Bemerkenswert ist, dass die Strategien und ihre Wirkungen vom Ergebnis her benannt werden, also als ein Produkt erfolgreicher Integrationspolitik, ${ }^{14}$ die es nun weiterzuführen gilt, und deren Fortschritte an Indikatoren gemessen werden sollen.

\section{Öffnung der Berufsausbildung als wichtiges Handlungsfeld}

Die zweite Handlungsstrategie umgreift auch die Öffnung von Berufsausbildung für Jugendliche (aus Familien) mit Einwanderungsgeschichte, die in den folgenden Kapiteln im Zentrum der Betrachtung steht. Übersetzt wird die Strategie unter der Überschrift »Integration durch Teilnahme am Erwerbsleben - Migranten*innen in der Ausbildung, auf dem Arbeitsmarkt und als Unternehmer*innen « in ein Ensemble von Handlungsfeldern und Leitprojekten, ${ }^{15}$ denen wiederum ein Satz von Zielen und Indikatoren zugeordnet ist. Das ist im Übrigen generell die Art und Weise, in der die Strategien im Integrationskonzept von 2007 konkretisiert und auf einer generellen Ebene operationalisiert werden.

Die Ausgangslage wird dabei so beschrieben: »Berufliche Orientierung und berufliche Lebensplanung müssen bereits frühzeitig, in Schule und mit dem Zugang zu qualifizierter Ausbildung, beginnen. Die Handlungsstrate-

13 Der Beauftragte des Senats von Berlin für Integration und Migration (Hg.): Vielfalt fördern-Zusammenhalt stärken. Das Berliner Integrationskonzept, Berlin 2007, S. 6.

$14 \mathrm{Zu}$ Aspekten der Evaluation von Integrationspolitik vgl. Kalman, Michael/Metje, Ute M./ Rolfes, Manfred/Kohlmeyer, Klaus: »Evaluation von Integrationspolitik. Bericht über die Frühjahrstagung von DeGEval mit einem Beitrag des damaligen Integrationsbeauftragten Piening«, https://www.researchgate.net/publication/297832061_Evaluation_von_Integrationspolitik_Fruhjahrstagung_de

15 Der Beauftragte des Senats von Berlin für Integration und Migration (Hg.): Vielfalt fördern-Zusammenhalt stärken. Das Berliner Integrationskonzept, Berlin 2007, S. $17 \mathrm{ff}$. 
gie Integration durch Teilnahme am Erwerbsleben setzt daher in der Schule und an allen drei kritischen Phasen der beruflichen Eingliederung an: Dem Übergang von der Schule in eine Ausbildung, dem Übergang von Ausbildung in eine Beschäftigung und der Wiedereingliederung ins Erwerbsleben nach vorangegangener Erwerbslosigkeit. In allen drei Phasen müssen die Förderungsinstrumente gezielt eingesetzt werden. Eine umfassende Strategie muss aber auch Möglichkeiten der Vernetzung von Angeboten und Akteuren zwischen den entscheidenden Stadien beruflicher Eingliederung aufzeigen. $\ll^{16}$

Damit ist ein ambitioniertes Programm skizziert, das implizit die Idee einer weiten Übergangsperiode und ihrer Unterstützung und Förderung enthält, sich also nicht auf die sogenannte erste Schwelle des Übergangs aus der Schule heraus reduziert. Zugleich nimmt es aber aus dem damaligen politischen Diskurszusammenhang die Zentrierung auf Ausbildung und die Konzentration auf kritische Übergangspunkte mit. Dies werden wichtige Aspekte der folgenden Kapitel sein.

\section{Handlungsfelder, Leitprojekte und Indikatoren}

Die Handlungsstrategie »Integration ins Erwerbsleben« wird in drei Handlungsfelder mit insgesamt sieben Leitprojekten übersetzt, dem vier Teilziele mit insgesamt sechs Indikatoren korrespondieren. Die Teilziele sind jeweils einem Oberziel zugeordnet, das explizit integrationspolitisch formuliert ist: "Migrant/-innen sollen gleiche Chancen beim Zugang zu Ausbildung und Erwerbstätigkeit eröffnet werden wie Angehörigen der Mehrheitsgesellschaft. 117 $^{17}$

16 Ebd., S. 17.

17 Ebd., S. 30. Vgl. zu den Schwierigkeiten bei der Verwendung des Begriffs »Mehrheitsgesellschaft«: »Mehrheitsgesellschaft ist ein gängiger Begriff, der missverständlich ist. Eigentlich müsste es heißen: Mehrheitsbevölkerung, also die von 64 Millionen Deutschen ohne Migrationshintergrund. In einem faktischen Einwanderungsland funktionieren Bezeichnungen wie `die deutsche Gesellschaft oder `die Gesellschaft in Deutschland «nicht als Synonym für Deutsche ohne Einwanderungskontext.« Neue deutsche Medienmacher e.V.: Glossar der Neuen deutschen Medienmacher. Formulierungshilfen für die Berichterstattung im Einwanderungsland, Berlin 2015. 
Eines der Handlungsfelder soll Ausbildung sein: "Es ist dringend erforderlich, deutlich mehr jugendliche Migranten/innen als bisher auszubilden. Der Schwerpunkt muss dabei auf die regulären Ausbildungsgänge gelegt werden. Reformen sollen sich daran orientieren, dass mehr Ausbildungsverhältnisse erfolgreich abgeschlossen werden, mit denen der Einstieg ins Erwerbsleben gelingt. $\ll^{18}$ Für beide Ziele, nämlich Einstieg in Ausbildung und erfolgreicher Abschluss einer Ausbildung werden quantitative Indikatoren angegeben, nämlich die Ausbildungsquote und erfolgreich abgeschlossene Ausbildungen, jeweils nach Anteil der Auszubildenden bzw. Absolventen mit und ohne Migrationshintergrund. 2007 war also ein wichtiger Prüfstein auf chancengleiche Ausbildung, nämlich der erfolgreiche Ausbildungsabschluss, schon im Blick, wenngleich die Aufnahme einer Arbeitsstelle, die ein Einkommen oberhalb der Bedürftigkeitsgrenze sichert, ein weiterer und lebensgeschichtlich entscheidender Prüfstein ist, der damals noch nicht formuliert worden war.

Für das Handlungsfeld »Integration in Ausbildung« werden des Weiteren zwei Leitprojekte identifiziert: Erstens die Förderung von Migrant*innen durch modularisierte Berufsausbildung und zweitens die Zertifizierung von Teilqualifizierungen und Erhöhung der Ausbildung im Öffentlichen Dienst. Während beim ersten Leitprojekt erhebliche Schwierigkeiten allein aufgrund der Tatsache zu erwarten waren, dass im geregelten System der dualen Berufsausbildung für jede Lösung das Einverständnis von Kammern, Betrieben und Gewerkschaften erforderlich ist, schien das zweite Leitprojekt - jedenfalls im ersten Anlauf - als rascher umsetzbar, weil mit dem Öffentlichen Dienst ein Adressat ins Auge gefasst wurde, von dem erwartet wurde, dass er senatsseitig gut erreich- und beeinflussbar sei.

\section{Integrativ gedacht, additiv entfaltet}

Die Gesamtanlage des Integrationskonzepts legt nahe, die verschiedenen Strategien, Handlungsfelder und Leitprojekte in einem interaktiven Zusammenhang zu betrachten, also ihre Isolierung nach Zuständigkeiten zugunsten kooperativer Bezüge aufzugeben. Für das Handlungsfeld »Integration

18 Der Beauftragte des Senats von Berlin für Integration und Migration (Hg.): Vielfalt fördern-Zusammenhalt stärken. Das Berliner Integrationskonzept, Berlin 2007, S. 18. 
in Ausbildung w war dabei nicht nur ein Zusammenspiel mit den anderen Handlungsfeldern im Bereich »Erwerbsleben« erforderlich, sondern eine enge Verbindung mit Handlungsfeldern der Strategie »Integration durch Bildung " geradezu zwingend.

Eine solche Vernetzung über die einzelnen Strategiefelder hinweg (und z.B. die Besetzung von Netzwerkknoten durch Leitprojekte) erfolgt im Integrationskonzept 2007 jedoch nicht; dass integrative Kooperationsgebot bleibt allgemein:»Eine Integrationspolitik, die [...] erfolgreich sein will, muss langen Atem haben. Sie muss angelegt sein als breites Feld von miteinander verzahnten Strategien, die auf die Verbesserung des Zugangs zu Bildung, Ausbildung und zum Arbeitsmarkt, auf die interkulturelle Öffnung der Regeldienste und auf eine bessere Partizipation von Migrant/-innen zielen $[\ldots ..] \times \ll^{19}$

Neben dieser mangelnden Verknüpfung der verschiedenen Strategiefelder bleibt im Integrationskonzept von 2007 auch die Begriffswahl noch weitgehend dem überkommenen Zeitgeist verpflichtet. Erst im Gesetz von 2010 wird neben »Integration « offiziell auch »Partizipation " gesetzt. Auf diese Weise wird begrifflich der Tatsache Rechnung getragen, dass ein großer Teil der Bevölkerung mit Migrationsgeschichte seit Jahrzehnten in Berlin lebt oder hier geboren und aufgewachsen ist, es also nicht um Integration, sondern um Teilhabe geht.

\section{Übergang Schule - Arbeitswelt integrationspolitisch schon vor 2007 Thema}

"Öffnung von Berufsausbildung « als integrationspolitisches Ziel hatte in der Zeit vor und um 2007 auch eine projektförmige Vorgeschichte. Der Arbeitsstab des Integrationsbeauftragten beteiligte sich am Programm »Kompetenzen fördern « des Bundesministeriums für Bildung und Forschung und des Europäischen Sozialfonds (ESF), und zwar in einem Teilfeld des Programms, ${ }^{20}$ das sich mit der Verbesserung der Bildungsbeteiligung von Ju-

19 Ebd., S. 3.

20 Bundesministerium für Bildung und Forschung: Modelle und Strategien zur Verbesserung der Bildungsbeteiligung von Jugendlichen mit Migrationshintergrund. Ergebnisse 
gendlichen mit Migrationshintergrund befasste und an zehn Standorten durchgeführt wurde. ${ }^{21}$

Dieses Projekt mit der Kurzbezeichnung BQN (»Berufliches Qualifizierungsnetzwerk für Migrantinnen und Migranten«) war in Anlage und Transferperspektiven breit aufgestellt. Zum Beispiel wurde eine Expertise zu "Kompetenzerfassung « in Auftrag gegeben und vorgelegt ${ }^{22}$ und ein Fachtag zur Sprachförderung für Migrantinnen und Migranten in der Schule durchgeführt. ${ }^{23}$ »Sicherung von Ausbildungserfolg « war ein weiteres exemplarisches Vorhaben, in dem es um die Analyse der Gründe und die Vermeidung von Ausbildungsabbrüchen am Beispiel von Zahnarzthelfer*innen und in Kooperation mit dem entsprechenden Oberstufenzentrum ging.

$\mathrm{Zu}$ den in Aussicht genommenen Transferperspektiven heißt es im entsprechenden Formblatt des Bundesministeriums für Bildung und Forschung: "In der Transferphase werden die Strukturen zur beispielhaften Entwicklung eines Landesprogramms gelegt, das die wichtigsten Aufgaben definiert. ${ }^{24}$

\section{Arbeitsmethode im Zentrum?}

Besonders hervorgehoben wird die als »BQN-Methode« bezeichnete Arbeitsweise. Der damalige Berater und Evaluationsbeauftragte von BQN und spätere Projektleiter von Berlin braucht dich! Klaus Kohlmeyer beschreibt sie an anderer Stelle, an der auch für ein Landesprogramm geworben wird. ${ }^{25}$

der Initiativstelle Berufliche Qualifizierung von Migrantinnen und Migranten (IBQM) beim Bundesinstitut für Berufsbildung (BIBB), Berlin 2006.

21 Das Berliner Projekt BQN, wurde damals beim Integrationsbeauftragten von Andreas Germershausen geleitet und von Klaus Kohlmeyer beraten. Es wurde nach Auslaufen dieser Förderung und ihrem Umbau »outgesourct« und als BQN e.V. zum Träger von Berlin braucht dich! (vgl. hierzu die folgenden Kapitel).

22 Der Beauftragte des Senats von Berlin für Integration und Migration: Expertise »Kompetenzerfassung «-FrauenComputerZentrumBerlin e.V. (=Berliner Beiträge zur Integration und Migration), Berlin 2006.

23 Der Regierende Bürgermeister. Senatskanzlei: Pressemitteilung vom 21.6.2006.

24 www.kompetenzen-foerdern.de/2522.php

25 Kohlmeyer, Klaus: Gutachten. Berlin: Europäische Modellmetropole für Integration und Vielfalt. Die schulische und berufliche Bildung junger Migrant/innen, Berlin 2006, S. $5 f$. 
Die Methode hat drei Bausteine, nämlich einen Satz aufeinander folgender Einzelschritte, Modellbildung und Exemplarik sowie dosierter Partnereinbindung und Verantwortungsgemeinschaft. ${ }^{26}$ Das Paket der aufeinander folgenden Einzelschritte besteht aus: »1. fundierter Problemanalyse durch einschlägige Experten/Expertinnen und Bewertung des Status Quo auf Basis eines 'gesicherten Integrationswissens`, 2 . Herausfiltern von tragfähigen Lösungsansätzen als Referenzmodelle und Ableiten von Arbeitsaufgaben für die relevanten Akteure, 3. Weiterentwicklung und Erprobung innovativer Lösungsansätze im Handlungsfeld und 4. Transfer erfolgreicher Ansätze in Politik und Praxis erst dann, wenn integrative Wirkungen nachweisbar sind.

Die Modellbildung soll sich entlang der biografischen Phasen der Berufseinmündung entwickeln; die Partnereinbindung soll nicht im Sinne von »alle-an-einen-Tisch«, sondern fokussiert und nach Entscheidungsebenen erfolgen. Dem Integrationsbeauftragten und seiner Dienststelle kommt in dieser Strategie eine Schlüsselstellung zu.

\section{Reformoptimismus ...}

In der Zusammenschau der im Kontext des Bundesprogramms formulierten Transferperspektiven für Berlin und der Skizze zur BQN-Methode zeigt sich in der Rückschau ein vermutlich zeitgebunden überschießender Reform-Optimismus, aber auch eine gewisse Überschätzung von Expertise und Expertentum. So konnte die Idee der Produktion eines »Gesicherten Integrationswissens « und eines daraus abgeleiteten wirksamen WissensManagements unter Qualitäts-Gesichtspunkten damals und später nur teilweise umgesetzt werden.

Insbesondere wird sich die Hoffnung auf ein Landesprogramm zur Integration von Migrant*innen im Prozess des Übergangs Schule - Beruf nicht erfüllen. Die Impulskraft von BQN und das Durchsetzungspotenzial des Integrationsbeauftragten - nicht als Person, sondern als Amt - werden überschätzt, die Schwierigkeiten, Widerstände und Hindernisse unterschätzt. Es kann aber durchaus sein, dass es genau dieser Optimismus war, für den viele Mitstreiter*innen gewonnen werden konnten.

26 »Verantwortungsgemeinschaft« wird zur selben Zeit zu einem Schlüsselkonzept der entstehenden Arbeitsgemeinschaft Weinheimer Initiative. 


\section{Integration und Desintegration im Wettlauf?}

Einen Einblick in die Ausgangslage, die am Ende des BQN-Vorhabens in Berlin erreicht war, gibt ein Nachhaltigkeitsreport, ${ }^{27}$ der neben Dokumentenanalysen auch auf einem »Lokaltermin« basierte, der in Berlin am 23. und 24. März 2006 stattfand.

Auf der einen Seite habe sich - so heißt es im Report - Aufmerksamkeit und Aktivität für die Förderung von Ausbildungs- und Berufsperspektiven junger Menschen mit Einwanderungsgeschichte erheblich verstärkt, auf der anderen Seite mehrten sich nach Ansicht der am Projekt in Berlin Beteiligten "Anzeichen von sozialer Desintegration, gerade bei Jugendlichen, die z.T. in den Medien weit über Berlin hinaus erhebliche Beachtung finden. Stichworte sind hier z.B. die >Rütli-Schule < und die so genannten >Neuköllner Verhältnisse , oder auch die Beachtung, die Filme wie `Knallhart in der allgemeinen Öffentlichkeit gefunden hätten. ${ }^{28}$ Es wird von einem »Wettlauf zwischen Integration und Desintegration « gesprochen. Vor diesem Hintergrund bestehe ein erheblicher Handlungsdruck.

\section{Idee: Integrationspolitischer Qualitätstreiber für den Übergang Schule - Beruf}

Vor dem Hintergrund dieses Spannungsverhältnisses zwischen Integration und Desintegration wurden die damals entwickelten Ansätze, die Förderung der Übergänge von der Schule in Ausbildung und Arbeitswelt aufzunehmen, als eine positive Antwort eingeschätzt. So auch der Tenor beim »Lokaltermin«: »Die BQN-Partner äußerten durchgehend ihre Zufriedenheit mit der von $B Q N$ eingeschlagenen Vorgehensweise, über Bestandsaufnahmen und exemplarische Erprobungen zu verbesserten Handlungsansätzen zu gelangen. Dies und die Trägerschaft des Vorhabens durch die Senatsdienststelle des Beauftragten für Integration und Migration markieren - folgt man den Diskussionsbeiträgen - diejenigen Punkte, durch die sich BQN von

27 Kruse, Wilfried: Nachhaltigkeitsreport. Lokale Netzwerke zur Berufsintegration von jungen Migrantinnen und Migranten am Ende der Projektförderung (=sfs-Beiträge aus der Forschung Band 158), Dortmund 2007.

28 Ebd., S. 75. 
der Vielzahl anderer Vorhaben in Berlin unterscheidet. $\aleph^{29}$ Dem Ansatz wird also insgesamt eine treibende Funktion zugetraut, insbesondere auch unter Qualitätsgesichtspunkten. Dies gilt insbesondere auch für das Handlungsfeld »Öffnung der Ausbildung im Öffentlichen Dienst«, in dem das Projekt durch die Bildung einer Steuerungsgruppe am Weitesten vorangekommen war. Bemerkenswert ist, dass schon damals die Einstellungsverfahren im Öffentlichen Dienst kritisch analysiert wurden; eine Perspektive, die erst Jahre später praktische Bedeutung erlangte. ${ }^{30}$

Auch die Frage nach einer wirksamen integrationspolitischen Koordinierung wird damals bereits aufgeworfen: »Denn eine Koordinierung, die lediglich eine politisch gewollte, aber im strikten Sinne unverbindliche Initiativfunktion wahrnimmt, wie es für das BQN-Vorhaben charakteristisch war, wird dem wachsenden Gestaltungsbedarf in einem sich ausdifferenzierenden, pluralen Übergangs-System [...] nicht gerecht. Es muss also nach übergreifenden Steuerungsstrukturen Ausschau gehalten werden, die der Gefahr einer Verbürokratisierung entgehen und dem BQN seine - aus der Integrationsproblematik sich legitimierende - treibende Funktion belassen. $\aleph^{31}$

Man kann also sagen, dass zum Zeitpunkt der Vorlage des Integrationskonzepts 2007 im Feld des Übergangs Schule-Arbeitswelt beim Integrationsbeauftragten ein erhebliches Problemverständnis entfaltet war, insbesondere auch in Hinblick auf Koordinierung, Steuerung, Qualitätssicherung und Institutionalisierung.

\section{Leitprojekt »Erhöhung der Ausbildung im Öffentlichen Dienst» ...}

Das Leitprojekt »Erhöhung der Ausbildung im Öffentlichen Dienst« ist im Grunde identisch mit der auf den Öffentlichen Dienst gewendeten und auf ihn konzentrierten Konzeption des damals beim Integrationsbeauftragten angesiedelten »Beruflichen Qualifizierungsnetzwerk für Migrantinnen und Migranten«. Gegenüber dessen breiterer Anlage stellt diese Konzentration zugleich eine Verengung dar.

\footnotetext{
29 Kruse, Wilfried: Nachhaltigkeitsreport, S. 97.

30 Vgl. Kapitel 8.

31 Kruse, Wilfried: Nachhaltigkeitsreport, S. 97.
} 
Die Konzentration auf den Öffentlichen Dienst hatte vor allem den Grund, dass hier der Anteil der Beschäftigten aus Einwandererfamilien besonders niedrig war. Aufgrund des seit 2002 bestehenden Einstellungsstopps im Öffentlichen Dienst des Landes konnte eine Erhöhung des Anteils nur über die Ausbildung erfolgen. Auch in der Ausbildung war der Anteil von Personen aus Familien mit Einwanderungsgeschichte sehr niedrig. Vor diesem Hintergrund wollte der Senat auch als Arbeitgeber Verantwortung übernehmen.

Folglich wurde neben der Praktikabilität und Aussicht auf einigermaßen rasche Erfolge im Integrationskonzept auf folgende Punkte hingewiesen: Der Öffentliche Dienst sei bei qualifizierten Jugendlichen mit Migrationshintergrund kein vorrangiges Berufsziel, Bewerber*innen seien zu wenig auf die Auswahlverfahren vorbereitet und diese zu selten nach dem fair-testVerfahren aufgebaut. Zudem werde der Umstand, dass ein »Bedarf an kulturspezifischen Kompetenzen« in der Verwaltung bestehe, bei den personalpolitischen Entscheidungen nicht berücksichtigt.

Das Leitprojekt konnte über diese Bestandsaufnahme hinaus auf Maßnahmen aufbauen, die bereits in der Pilotphase eingeleitet waren: die Bildung eines Steuerungsgremiums zum Thema »Ausbildung von Migranten"innen im Öffentlichen Dienst « und der Umstand, dass bereits seit 2005 bei den Ausschreibungen für Ausbildungsplätze ein Zusatz eingefügt wurde, der lautet: »Wir begrüßen ausdrücklich Bewerbungen von jungen Menschen nichtdeutscher Herkunft, die die Voraussetzungen erfüllen.« Der Rahmen war damit aufgespannt und eine Reihe von Problematiken schon angesprochen, die in den nächsten Jahren eine erhebliche Rolle spielen sollten, wie z.B. die Einstellungsverfahren.

\section{... und seine Umsetzung als Kampagne Berlin braucht dich!}

2008 wurde die Umsetzung der Kampagne zur weiteren Öffnung der Ausbildung im Öffentlichen Dienst für Jugendliche mit Migrationshintergrund ausgelagert. Ein externer Träger wird gegründet und betreibt unter dem schon eingeführten Namen BQN im Auftrag des Integrationsbeauftragten ausschließlich Berlin braucht dich!. Auf diese Weise sind die im Zuge der vorherigen Projektarbeit aufgebauten Kompetenzen und die entstandenen $\mathrm{Zu}$ gänge für die weitere Arbeit gesichert. 
Startete das Vorhaben zunächst mit dem Öffentlichen Dienst, so kamen 2009 Unternehmen, an denen das Land beteiligt ist, im Folgenden »Öffentliche Unternehmen" genannt, hinzu. Die Entscheidung für eine Konzentration auf den Öffentlichen Dienst und die Öffentlichen Unternehmen war in verschiedener Hinsicht folgenreich. Dies wird in den folgenden Kapiteln immer wieder zum Thema. Zu Beginn von Berlin braucht dich! war noch unentschieden, wie die Konzentration auf den Öffentlichen Dienst zu verstehen ist: als ein zeitlich begrenzter Öffnungsimpuls, der beispielhaft und transferorientiert auch für andere Wirtschaftssektoren der Stadt steht, ${ }^{32}$ oder als langfristig angelegte >Durcharbeitung des Öffentlichen Dienstes und der Landesbetriebe unter der integrationspolitischen Leitfrage nach gleichen Chancen für junge Menschen mit Migrationsgeschichte. Damit würde auch die Chance bestehen, die Nachfrageseite selbst als ein - kooperatives - Gestaltungsfeld zu sehen und - im Sinne von Querschnittspolitik - Verbindung zu arbeitspolitischen Ansätzen, wie z.B. Diversity, herzustellen.

Die dahinterliegende politische Frage war, ob genügend Zeit ist oder eingeräumt werden kann, die jetzt erfolgende Öffnung der Nachfrageseite (Arbeitgeber des Öffentlichen Dienstes) als exemplarisches Beispiel guter Politik zu nutzen und auszubauen, oder ob es angesichts eines sich anbietenden Zeitfensters und damit der günstigen Gelegenheit eher angeraten sei, die Aktivitäten schnell auszuweiten, um aus Berlin braucht dich! eine prominent sichtbare stadtweite Aktion zu machen.

Die Entscheidung fiel in den ersten folgenden Jahren immer wieder zugunsten der weiteren Konzentration auf den Öffentlichen Dienst und die Öffentlichen Betriebe aus. Erst später erfolgte eine Erweiterung zu Betrieben des Metall- und Elektrosektors und punktuell zu weiteren großen Ausbildungsbetrieben anderer Branchen.

Auf jeden Fall aber ist - auch durch die erheblichen Ressourcen, die in Berlin braucht dich! gehen - Berufsausbildung als ein besonders wichtiges integrationspolitisches Handlungsfeld gesetzt. Konfrontiert ist Integrationspolitik darin mit der »dualen Berufsausbildung « und ihrer starken Stellung der ausbildenden Betriebe u.a. mit dem Umstand, dass die Betriebe ent-

32 Kruse, Wilfried: Berlin braucht dich! - über den Öffentlichen Dienst hinaus? Eine Recherche zu Integration und dualer Berufsausbildung. Expertise für BQN Berlin/Dortmund 2010. Auch als PDF: https://www.bqn-berlin.de/site/assets/files/1140/bqn_berlin_expertise_ausbildung.pdf 
scheiden, wem sie einen Ausbildungsvertrag geben. Das ist ein wesentliches rechtliches und faktisches Merkmal des deutschen Berufsbildungssystems mit weitreichenden Folgen. Nicht nur die fachlich-inhaltliche, sondern vor allem auch die übergangsbiografische und kooperative Ausgestaltung dieses Felds wird damit zur zentralen Gestaltungsherausforderung.

Abbildung 1: Plakat »Erleuchtung«

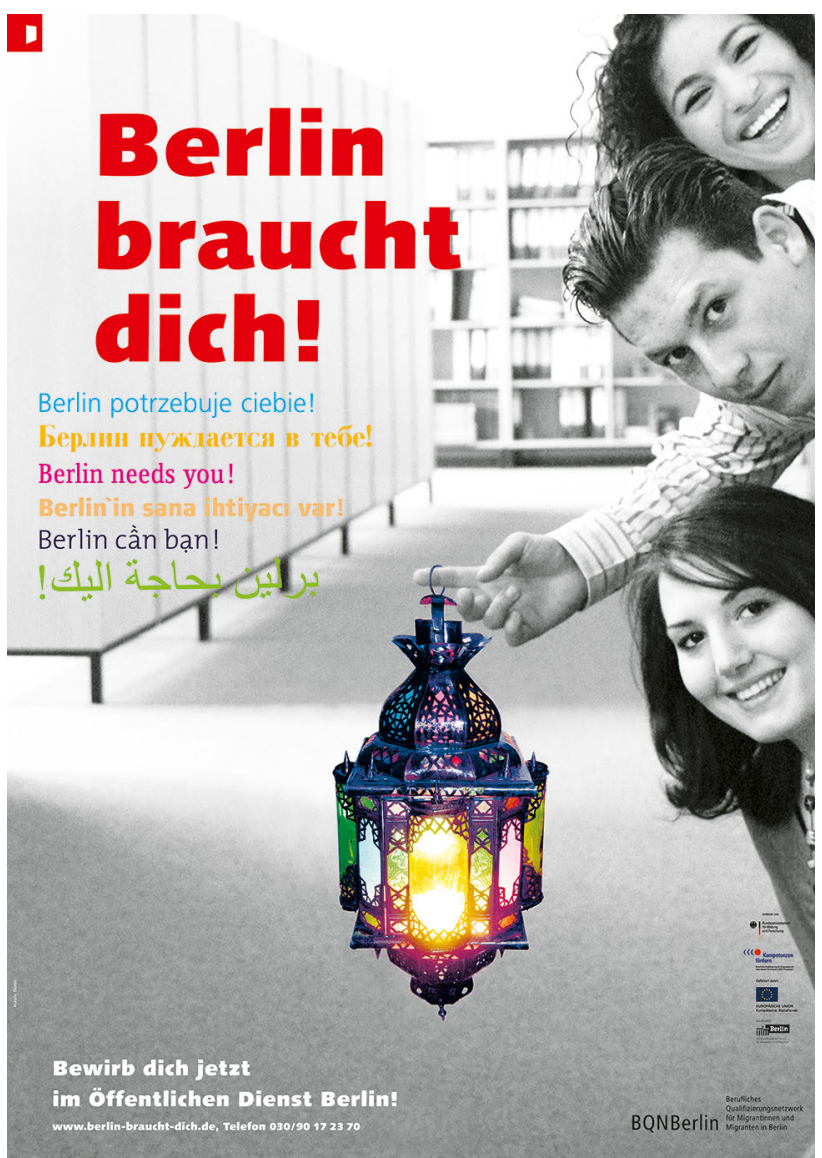

Copyright: kursiv Kommunikationsdesign, Katrin Schek; Foto: Barbara Dietl. 


\section{Fazit}

In diesem ersten Kapitel wurde gezeigt, wie das integrations- und teilhabepolitische Ziel der Öffnung von Berufsausbildung für junge Leute mit Migrationshintergrund oder - wie wir heute sagen würden - aus Familien mit Einwanderungsgeschichte in die Erneuerung der Berliner Integrationspolitik seit 2007 eingebettet ist. »Öffnung der Berufsausbildung« war und ist nur ein Handlungsfeld von Integrationspolitik, aber eines mit erheblicher Bedeutung, sowohl hinsichtlich seiner konkreten und tatsächlichen Ausgestaltung als auch in einem exemplarischen Sinne als Feld, in dem sich Integration oder Teilhabe als Politik mit ihren Entscheidungen, ihren Optionen, Widerständen und Widersprüchen ausbuchstabiert.

Das Integrationskonzept von 2007 markiert die definitive Abkehr von der bisherigen "Ausländerpolitik« zugunsten eines komplexen und handlungsorientierten integrationspolitischen Ansatzes für die Einwanderungsstadt Berlin. Deutschland befindet sich seit Ende der 1990er Jahre auf dem Weg zu einer erneuerten Integrationspolitik. Berlin nimmt insofern keine Sonderrolle ein, geht aber anderen Bundesländern bei der Konkretisierung von Integrationspolitik, ihrer politisch-institutionellen Verankerung als Querschnittsaufgabe und der Verabschiedung eines Partizipations- und Integrationsgesetzes im Jahr 2010 voraus. Mit der Doppelbezeichnung »Partizipation« und "Integration« wird im Übrigen dem Umstand Rechnung getragen, dass die Mehrheit der Menschen mit Einwanderungsgeschichte schon viele Jahre oder gar Jahrzehnte in Berlin leben oder sogar hier geboren und aufgewachsen sind; es also nicht um Integration, sondern um Teilhabe geht. Denn auch begrifflich muss das veränderte Verständnis - von der Anwesenheit zur Zugehörigkeit - zum Ausdruck gebracht werden, was nur allmählich und teilweise noch unbefriedigend gelingt.

Das Integrationskonzept beinhaltet in seiner handlungsorientierten Anlage über Handlungsfelder, Leitprojekte und Indikatoren eine strategische Vorgehensweise, in der Leitprojekten für die jeweiligen Handlungsfelder eine Hebelfunktion zugeschrieben wird. Im Rahmen der Handlungsstrategie »Integration ins Erwerbsleben« wird nun als ein Handlungsfeld Ausbildung identifiziert, als zentraler Indikator der Anteil von Auszubildenden mit Migrationshintergrund genannt und die Erhöhung des Anteils in der Ausbildung im Öffentlichen Dienst als ein Leitprojekt eingeführt. 
Auch die Aufmerksamkeit auf die Öffnung der Berufsausbildung für junge Menschen aus Familien mit Migrationsgeschichte ist keine Berliner Besonderheit, denn die kritische Situation am Übergang von der Schule in die Berufsausbildung hatte schon seit geraumer Zeit zu Aktivitäten auf kommunaler Ebene geführt und war Gegenstand von Förderprogrammen verschiedener Bundesländer und des Bundes. An einem einschlägigen Förderprogramm des Bundesbildungsministeriums zur Netzwerkbildung zwischen den Akteuren im Übergang war der Berliner Integrationsbeauftragte selbst mit einem Projekt beteiligt.

Die Besonderheit des Berliner Ansatzes ist darin zu sehen, dass die Entscheidung getroffen wurde, bei den integrationspolitischen Bemühungen um eine Öffnung der Berufsausbildung den Öffentlichen Dienst zu priorisieren. Neben der Tatsache, dass der Anteil von Jugendlichen aus Familien mit Migrationsgeschichte im Öffentlichen Dienst zum damaligen Zeitpunkt sehr niedrig war, wurde für diese Prioritätenentscheidung ausschlaggebend, dass der Öffentliche Dienst politischen Vorhaben zugänglich ist und Berlin mit dem eigenen Öffentlichen Dienst eine Vorreiter- und Vorbildfunktion wahrnehmen könnte. Dies mündete in eine Kampagne der Öffentlichen Arbeitgeber, die unter dem Motto »Berlin braucht dich!« junge Menschen mit Migrationshintergrund motivieren sollte, sich beim Öffentlichen Dienst zu bewerben. Als erster Schritt der Umsetzung des im Integrationskonzept formulierten Leitprojekts stieß Berlin braucht dich! auf großes Interesse und löste tatsächlich einen Anstieg des Anteils von Auszubildenden mit Migrationshintergrund im Öffentlichen Dienst und bei den später hinzukommenden Betrieben mit Landesbeteiligung aus.

In der Rückschau zeigt sich, dass diese Entscheidung für die Priorisierung des Öffentlichen Dienstes weitreichende und zum Teil widersprüchliche Konsequenzen hatte. Hiervon ist den folgenden Kapiteln die Rede. Aber auch die Anlage der Kampagne zeigt eine gewisse Einseitigkeit, weil ihre Hauptadressaten nicht auch die Betriebe sind, sondern primär junge Menschen als potenzielle Bewerber"innen für einen Ausbildungsplatz im Öffentlichen Dienst. Insofern teilt die Kampagne bei ihrem Start den »Zeitgeist«, vor allem auf die Motivationen und die mitgebrachten Kompetenzen der jugendlichen Bewerber*innen zu blicken und weniger auf eine an Vielfalt orientierte und ihr förderliche betriebliche Ausbildungsgestaltung. Damit ist eine weitere schwierige Problematik benannt, die in den Folgejahren die Entwicklung des Leitprojekts beeinflussen wird. 
Abbildung 2: Plakat zu »Bewirb dich jetzt beim Land Berlin«

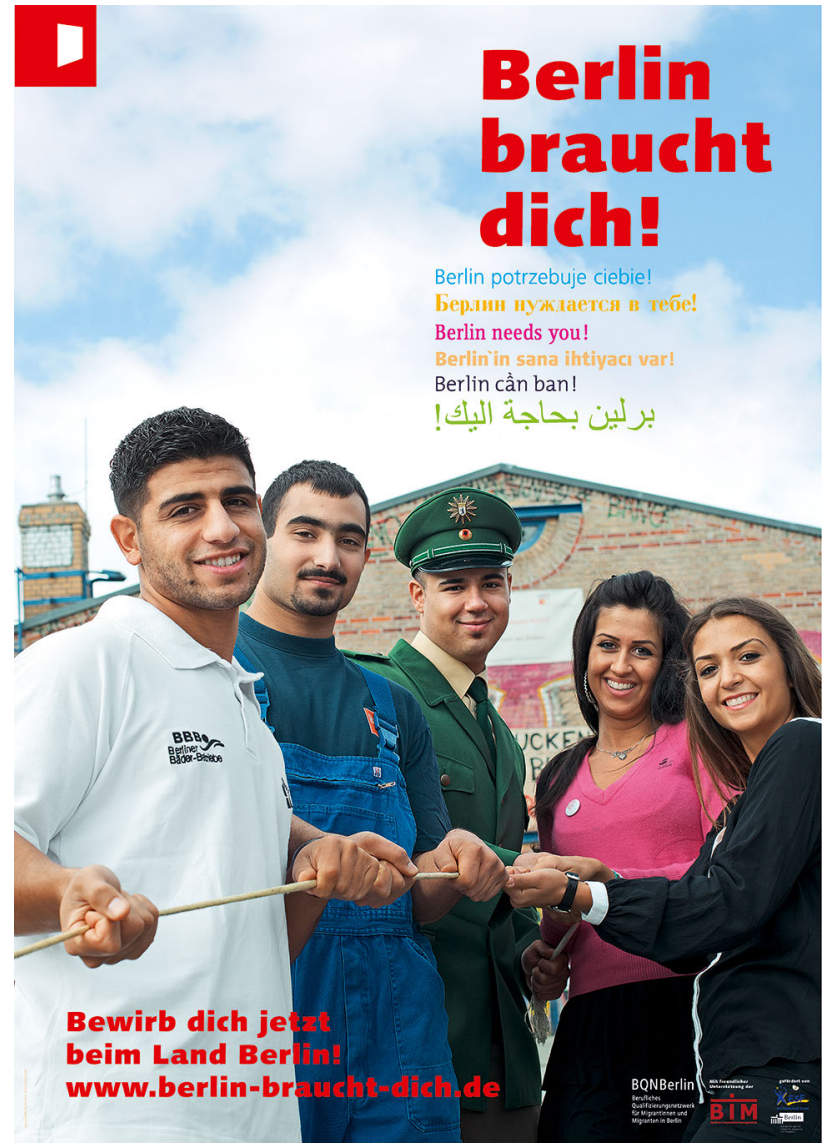

Copyright: kursiv Kommunikationsdesign, Katrin Schek; Foto: Barbara Dietl.

Schließlich wird gerade auch dann, wenn man den Blick stark auf die jungen Menschen und deren individuelle Voraussetzungen für eine erfolgreiche Ausbildung richtet - was eben in sich schon zu einseitig ist - eine Schwäche des Integrationskonzepts von 2007 deutlich: nämlich die fehlende konzeptionelle und operative Verknüpfung zwischen den beiden Handlungsstrategien »Integration ins Erwerbsleben« und »Integration durch Bildung«. Damit ist ein integrationspolitisches Grundsatzproblem aufgeworfen, das 
38 Ausbildung statt Ausgrenzung

als Frage der Ausgestaltung von Integration als Querschnittsaufgabe in den folgenden Kapiteln immer wieder zur Sprache kommt. 


\section{Kapitel 2}

\section{Was ein Integrationsbeauftragter überhaupt bewegen kann}

Im Land Berlin sah man schon frühzeitig, dass es notwendig ist, der Tatsache eines großen Anteils von Menschen mit Migrationsgeschichte an der Bevölkerung Rechnung zu tragen. ${ }^{1} 1981$ richtete der damalige Regierende Bürgermeister in der noch geteilten Stadt, Richard von Weizsäcker (CDU), deshalb die Stelle einer Ausländerbeauftragten ein und besetzte sie mit Barbara John. Neben den sogenannten »Gastarbeitern« kamen ab 1976 zunehmend Flüchtlingsgruppen in den Westteil der Stadt. Die Zahl der Ausländer lag zum Zeitpunkt der Wiedervereinigung 1989 in Berlin-West bei 296.620 Personen, womit diese einen Anteil von 15,9 Prozent an der Gesamtbevölkerung hatten. Einen deutlichen Schwerpunkt bildete die Bevölkerungsgruppe mit türkischem Hintergrund. ${ }^{2}$

1 Vgl. dazu auch: Häussermann, Hartmut/Kapphan, Andreas: Berlin - von der geteilten zur gespaltenen Stadt? Sozialräumlicher Wandel seit 1990, Wiesbaden 2002.

2 Vgl. auch die sehr detaillierte Studie Gyapay, Borbála: »Die Veränderung des ethnischen Bildes Berlins«, in: Zeitschrift für amtliche Statistik Berlin Brandenburg 3/2012, S. 46-55. 


\section{Neustart, aber immer noch: Integration}

Auch die Umbenennung des Amts in Beauftragter für Integration und Migration mit dem Wechsel zu Günter Piening im Jahr 2003 signalisiert die sich allmählich sich durchsetzende Einsicht in den Tatbestand »Einwanderungsstadt«. ${ }^{3}$

Die Benennung als Integrationsbeauftragter ist sicherlich dem damaligen politischen Zeitgeist geschuldet und hat sich dann gewissermaßen »eingebürgert«. Die Bezeichnung transportiert noch ein Verständnis von Migration, dem eine Art polares Schema zugrunde liegt: Menschen, die zu uns kommen, treffen auf eine kulturell homogene - d.h. mehr oder weniger deutsche - Mehrheitsgesellschaft, in die hinein der Integrationsprozess verläuft und zu gestalten ist.

Dieses Verständnis ist heute nicht mehr zeitgemäß, denn in den Jahrzehnten, in denen Deutschland immer mehr zu einer Einwanderungsgesellschaft geworden ist, trifft das polare Schema nicht mehr die soziale Wirklichkeit einer dauerhaft heterogenen Gesellschaft. Im Partizipations- und Integrationsgesetz des Landes Berlin von 2010 wurde dagegen das Konzept der Partizipation stark gemacht. Es unterlegt heute den Begriff Integration mit einem anderen Verständnis, nämlich eines der gleichberechtigten Teilhabe. Diese Teilhabe-Sicht wurde von Piening als neuen Integrationsbeauftragten stark betont. Sie zieht sich als Leitidee durch die Integrationspolitik der folgenden Jahre. ${ }^{4}$

3 Zu diesem »Neustart« der Berliner Integrationspolitik vgl. auch: Cesemann, Frank: Berlin: »Einwanderungsstadt sunder construction ?? Von der Beauftragtenpolitik zur strategischen Steuerung«, in: Frank Cesemann/Roland Roth (Hg.): Lokale Integrationspolitik in der Einwanderungsgesellschaft, Wiesbaden 2009, S. 311-333.

4 Die bisherigen Berliner Beauftragten waren: 1981-2003 Barbara John, 2003-2012 Günter Piening, 2012-2015 Dr. Monika Lüke, 2015-2019 Andreas Germershausen, seit Mai 2019 Katarina Niewiedzial. 


\section{Integrationsbeauftragter: Institutionelle Einbindung}

Mit Namen und Verständnis verändert sich auch die >Einhängung des Themas im Berliner Senat. Denn in den Jahren, in denen Barbara John als Ausländerbeauftragte tätig war, gab es keine Senatsverwaltung, die Integration ausdrücklich zum Gegenstand hatte. Aber auch John war der Sozialsenatorin zu- und in allen Verwaltungsdingen der Staatssekretärin für Soziales nachgeordnet.

Als der zweite rot-rote Senat im Jahr 2006 mit der Senatsverwaltung für Integration, Arbeit und Soziales erstmals ein Senatsressort zum Thema einrichtete, geschah dies, um »Integration« als politisches Handlungsfeld aufzuwerten. Wohl nicht intendiert waren zwei Folgerungen dieser Entscheidung: Erstens gibt es seitdem neben dem/der Beauftragten auch eine Senatorin und eine*n Staatssekretär*in für Integration. Zwar war auch zuvor schon die jeweilige Senatorin oder der jeweilige Senator politisch-fachlich mitverantwortlich für die Aktivitäten der Ausländerbeauftragten. Als Integrationssenatorin muss sie jedoch selbst das Thema vertreten. Daraus haben sich Konkurrenzen gerade in der Pressearbeit ergeben, die - je nach dem Charakter der jeweiligen Senatorin - unterschiedlich intensiv ausfielen. Zweitens gerieten mit der Schaffung des Integrationsressorts Dissense um die Ausländer- und Integrationspolitik zu Konflikten im Senat. Da der Senat - so eine goldene Regel - stets mit einer Stimme spricht, sind langdauernde Dissense möglichst auszuschließen. Im Ergebnis der Aufwertung des Themas als ein Senatsressort war die Rolle des oder der Integrationsbeauftragten neu zu klären.

Nach den Integrationskonzepten von 2005 und 2007 sowie der Schaffung des Senatsressorts 2006 ist die Verabschiedung des Partizipations- und Integrationsgesetzes von 2010 (PartIntG) eine dritte Aufwertung des Themas in der Senatspolitik. Im Gesetz, das auf eine Forderung des Landesbeirats für Integrations- und Migrationsfragen zurückgeht, wird die fachliche Aufgabenstellung des/der Integrationsbeauftragten erstmals gesetzlich festgeschrieben.

Die umfassenden Querschnittsaufgaben, die dort formuliert sind, machen aber eine gewisse Eigenständigkeit des/der Integrationsbeauftragten erforderlich. Querschnitt, also Setzung des Themas für den gesamten Senat, Eigenständigkeit der/des Beauftragten und Einbindung in eine Verwal- 
tungsstruktur bilden ein Konfliktfeld, das die Abfassung des Gesetzes bestimmte. Dabei waren zwei Aspekte vorrangig: Erstens das Ressortprinzip, nach dem jede Senatorin, jeder Senator volle Verantwortung für die jeweilige Verwaltung hat. Insofern war die Reduzierung des Querschnittsansatzes und die Zuschreibung in die Verantwortung des/der für Integration zuständigen Senators*in eine Abwehr anderer Verwaltungen, insbesondere seitens des Innensenators und der Justizsenatorin, gegen eine befürchtete Dominanz der Integrationspolitik. Zweitens ist der Senat für je eine Legislaturperiode gewählt, während die/der Integrationsbeauftragte eine Verwaltungsposition innehat. Folglich ist es plausibel, dass das Gesetz die Eigenständigkeit des/der Beauftragten einschränkt und die Funktion als »im Auftrag des/der zuständigen Senator*in handelnd« definiert.

Allerdings kann die Eigenständigkeit des Beauftragten durch die politischen Akteur*innen unter Druck geraten; zuletzt nach der Regierungsbildung von SPD und CDU Ende 2011. Auch blieb in der Koalition von 2011 bis 2016 das eigene Presserecht des Integrationsbeauftragten nur bestehen, da es im Partizipations- und Integrationsgesetz (PartIntG) festgeschrieben war. Festzuhalten ist dass die Eigenständigkeit des Beauftragten in den gegebenen politischen Strukturen einen Goodwill seitens der je zuständigen Senatorin voraussetzt und zugleich, dass die gesetzliche Festschreibung der Rolle des Beauftragten ein nicht zu unterschätzendes Positivum ist.

Die Aufgabe des/der Beauftragten für Integration und Migration ${ }^{5}$ wird im Gesetz zur Regelung von Partizipation und Integration in Berlin vom 28.Dezember 2010 sehr anspruchsvoll formuliert: »Die Hauptaufgabe der/ des Beauftragten des Senats für Integration und Migration besteht darin,

5 Als »weitere« Aufgaben werden auf der aktuellen Homepage genannt: »Weitere Arbeitsschwerpunkte sind die: Zusammenarbeit mit und Unterstützung von Migrantenorganisationen, Interkulturelle Öffnung der Verwaltung, Beratung in zuwanderungs- und integrationsrechtlichen sowie sozialen Fragen, Informations- und Beratungsangebote im Willkommenszentrum Berlin für alle neu Zugewanderten zu Themen, die ihr Ankommen und ihren Aufenthalt in Berlin betreffen, Migrations- und Flüchtlingspolitik, Menschenrechte und ethnische Minderheiten, Grundsatzfragen der sozialen und gesellschaftlichen Integration von Spätaussiedlerinnen und Spätaussiedlern, Geschäftsstelle des 2003 gegründeten Landesbeirats für Integrations- und Migrationsfragen, in dem Vertreterinnen und Vertreter von Migrantenorganisationen neben Vertretern der Senatsverwaltungen, der Wirtschaft und Wohlfahrtseinrichtungen die Migrationspolitik des Senats kritisch begleiten und weiterentwickeln, Informations- und Öffentlichkeitsarbeit« https://www. berlin.de/lb/intmig/ 
die Migrations- und Integrationspolitik des Senats von Berlin mitzugestalten und mit anderen Verwaltungen im Land Berlin abzustimmen. Da Integration eine Querschnittsaufgabe ist, d.h. alle Politikfelder und damit alle Senatsverwaltungen gleichermaßen hierzu für ihren Bereich Verantwortung tragen, obliegt der/dem Integrations- und Migrationsbeauftragten die strategische Steuerung der gesamten Integrationspolitik Berlins. ${ }^{6}$

Berlin ist das erste von bisher drei Bundesländern mit einem Integrationsgesetz. Außer Berlin verfügt noch NRW seit 2012 und Baden-Württemberg seit 2015 über ein solches Gesetz. »Die bisher vorliegenden Gesetze zur Teilhabe von Menschen mit Migrationshintergrund sind als ein wichtiger Schritt für die Integrationsarbeit anzusehen. Es ist ihr Verdienst, zum ersten Mal auch die Aufgaben und Pflichten der aufnehmenden Gesellschaft für eine gelingende Integration in den Blick zu nehmen. «"

In ihrer Darstellung der Integrationsgesetzte schätzen Karin Weiß und Birsin Alan die Ausstattung der für das Berliner Gesetz zentralen Figur des/ der Integrationsbeauftragten kritisch ein:

Bemerkenswert ist, dass in Berlin zwar die Position des/der Integrationsbeauftragten des Senats gesetzlich verankert ist, diese/r aber ausdrücklich als sim Auftrag handelnd - und damit weisungsgebunden - definiert wird. Es wird hier ebenfalls geregelt, dass alle die Integration betreffenden Cesetze, Verordnungen oder andere wichtige Vorhaben der Senatsverwaltung dem/der Beauftragten zur Stellungnahme vorgelegt werden müssen. Ein Einspruchsrecht ergibt sich daraus nicht. Damit ist der/die Berliner Beauftragte zwar als Position abgesichert, aber im Konfliktfall ohne jede Durchsetzungskraft. ${ }^{8}$

Weiß/Alan sprechen zu Recht das Spannungsfeld an, das sich zwischen Eigenständigkeit und Weisungsgebundenheit auftut, berücksichtigen aber nicht die Voraussetzungen, die sich aus durchaus üblichen parlamentari-

\footnotetext{
6 http://gesetze.berlin.de/jportal/portal/t/6ez/page/bsbeprod.psml?pid=Dokumentanzeige\&showdoccase $=1 \&$ js_peid=Trefferliste\&documentnumber $=1 \&$ numberofresults $=10 \&$ fromdoctodoc $=y e s \& d o c . i d=j \mid r-$ PartIntergrCBErahmen\&doc. part $=X \& d o c$. pri$\mathrm{ce}=0.0 \&$ doc. $\mathrm{hl}=1$

7 K. Weiß, B. Alan: »Integrationsgesetze in den Bundesländern«, S. 272.

8 Ebd., S. 271.
} 
schen Strukturen ergeben, die - wie dargelegt - die politische Gestaltungsmacht einer Landesregierung nicht beschränken dürfen.

Zudem kann man die Formulierung »im Auftrag handelnd « auch politischer interpretieren und als eine Art politisches Mandat im Namen des Senats verstehen. Es ist allerdings schwer vorstellbar, dass für die Aufgabe einer "strategischen Steuerung der gesamten Integrationspolitik Berlins« das bisherige institutionelle Arrangement angemessen sein soll. Denn genau das, nämlich den Aufbau, die Stabilität und die Weiterentwicklung strategischer Kooperation quer zu den tief verankerten vertikalen Hierarchien in den einzelnen Verwaltungseinheiten erweist sich als größte Herausforderung für die Rolle eines/einer Integrationsbeauftragten. Zudem birgt die Kopplung der verwaltungsinternen Funktion Abteilungsleiter mit der öffentlichkeitsbezogenen Funktion Integrationsbeauftragte/r die Gefahr spannungsreicher struktureller Überforderungen in sich. ${ }^{9}$

\section{Integration und Teilhabe als Querschnittsaufgabe}

Die Anerkennung der Tatsache einer schon Jahrzehnte währenden Einwanderung erfordert es, innerhalb der mittlerweile großen Bevölkerungsgruppe mit Migrationshintergrund zwischen Generationen von Einwanderern und Typen von Einwanderung zu unterscheiden.

Viele Menschen, die aus Familien stammen, die eingewandert sind, sind schon in Deutschland geboren und aufgewachsen und sehen Deutschland als ihre Heimat an. Die immer gebräuchlicher werdende Bezeichnung »Menschen mit Migrationsgeschichte« oder auch die Debatte über »PostMigration « versuchen, ${ }^{10}$ diesen Sachverhalt einzufangen. Es kommen aber weiterhin Menschen nach Deutschland, die dies mit einer Einwanderungsperspektive verbinden. Hier sind besonders die Asylsuchenden zu nennen,

9 Die öfentlichkeitsbezogene Funktion wird durch ein eigenständiges Presserecht des Beauftragten gestärkt, die im Partizipations- und Integrationsgesetz geregelt ist. Die seit Ende 2016 regierende rot-rot-grüne Koalition hat die Eigenständigkeit des Beauftragten politisch wieder gestärkt, die erläuterte spannungsreiche Überforderung aber nicht aufgehoben.

10 Vgl. Foroutan, Naika: »Die postmigrantische Cesellschaft«, www.bpb.de/gesellschaft/ migration/kurzdossiers/205295 am 21.4.2015. 
weil der Umgang mit ihnen gewissermaßen eine Nagelprobe für das soziale und politische Selbstverständnis von Deutschland als Einwanderungsland ist. Wie mit Geflüchteten umgegangen wird, wirkt insofern auch in vielerlei Hinsicht auf die hier lebenden Menschen mit Migrationsgeschichte zurück.

Integrations- bzw. Teilhabepolitik wird vor diesem Hintergrund zur Querschnittsaufgabe, und zwar sowohl der Sache als auch der Form nach. Der Sache nach heißt das, dass aus der heutigen sozialen Struktur unserer Gesellschaft und deren fortlaufende Veränderung folgt, dass es kein Handlungsfeld gibt, welches unabhängig vom Faktum der Einwanderungsgesellschaft betrachtet werden kann. Beispiele können beliebig gewählt werden, sei es Stadtentwicklung, Wohnen, Schule und Ausbildung, Kultur. Die Tatsache der Einwanderungsgesellschaft durchprägt alle Handlungsfelder.

Der Form nach ist es also erforderlich, eine Vorgehensweise strategischer und kooperativer Verknüpfungen zu finden, die nicht zu Sondersystemen führt, die ihrerseits oftmals diskriminierende Effekte haben. Diese neue Vorgehensweise kann man als Querschnittsstrategie bezeichnen - oder auch als Fokuspolitik. ${ }^{11}$ Nach diesem Verständnis ist das Amt Integrationsbeauftragte/reine Fokusagentur.

\section{Fokus Migration: eine konzeptionelle Idee ...}

$»$ Fokus Migration $\aleph^{12}$ meint eine bestimmte Herangehensweise an ein Handlungsfeld; sie setzt sich von einer in der Vergangenheit vielfach üblichen Weise ab, Integration/Migration als einen gesonderten Förderbereich zu behandeln, der seine Begründung in einer als prinzipiell angenommenen und quasi »naturalisierten« Benachteiligungslage von Menschen mit Migrationsgeschichte hatte. Dieses Verständnis führte oft feststellbare Benachteiligungen implizit oder explizit auf Defizite bei den betroffenen Menschen mit Migrationsgeschichte zurück, während strukturelle Benachteiligungen

11 Vgl. hierzu auch: Kruse, Wilfried: Cut Ankommen in der Arbeitswelt. Expertise für den Beauftragten des Berliner Senats für Integration und Migration, Unveröffentlichtes Manuskript, Dortmund 2013.

12 Kohlmeyer, Klaus/Pavkovic, Gari: »Fokus Migration und die kommunale Steuerung am Übergang Schule und Beruf«, in: Arbeitsgemeinschaft Weinheimer Initiative (Hg.): Lokale Bildungsverantwortung, Stuttgart 2013, S. 247- 261. 
oder Diskriminierungen und Rassismus mit ihren Folgen weniger in den Blick kamen.

Dies alles begründet außerdem, warum Integrationspolitik und ihre jeweiligen Prioritäten einer steten Überprüfung bedürfen. Der Fokus-Ansatz lenkt daher die Aufmerksamkeit auch auf eine fortlaufende Analyse von Bedingungen und Prozessen der Entstehung von Benachteiligung, ihrer Verfestigung, ihrer Verringerung und ihrer Auflösung.

Für das Beispiel Übergang von der Schule in die Arbeitswelt bedeutet dies, dass der "Fokus Migration" nicht ein spezielles Handlungsfeld neben anderen ist, sondern als Querschnittsfrage alle Handlungsfelder beleuchten soll, eben als Fokus. Er lenkt die Aufmerksamkeit in jedem denkbaren Handlungsfeld (also z.B. schulische Berufsausbildung, Bewerbungs- und Auswahlverfahren, Gestaltung von Berufsausbildung, Jugendhilfe usw.) auf die Frage, ob Teilhabemängel für Jugendliche mit Migrationsgeschichte - in Relation zu Jugendlichen mit einheimischer Herkunftsgeschichte - faktisch feststellbar sind und worauf dies zurückzuführen ist. Was zu tun ist, ergibt sich so nicht aus vermeintlichen Selbstverständlichkeiten oder unhinterfragter Fortschreibung bisheriger Praxis und auch nicht aus einer statisch gesetzten Benachteiligungsannahme. Maßnahmen, die zu entwickeln sind, müssen Teilhabemängel insgesamt adressieren und darin ggf. sichtbar gewordene spezifische Problematiken, die auf »Migration« zurückgehen, aufgreifen. Welche dies sind, muss Ergebnis von handlungsorientierten Analysen sein.

Es entsteht auf diese Weise ein Bild von Handeln, das einer zyklischen Bewegung folgt, die aus Praxis, Überprüfung, fortlaufender Analyse und immer wieder erneuerter Konsensbildung über Prioritätensetzung und deren Verfolgung besteht.

\section{... und ihre Aufgaben}

Breite, Differenziertheit und Schwierigkeitsgrad von dem, was mit »Integration als Querschnittsaufgabe« gemeint sein kann, sind enorm. Der »Fokus « soll die Fachpolitiken unter dem Aspekt von Migration und Integration beleuchten. Die fachliche Verantwortung verbleibt jedoch bei den jeweiligen Fachpolitiken. Für die Kooperation von Integrations- und Fachpolitik bedarf 
es daher angemessener Organisationsformen, um den »Fokus Migration« in die jeweiligen Fachpolitiken einzuarbeiten.

Basis hierfür ist, dass die jeweilige Fachlichkeit und Verantwortung respektiert werden und dass die »Fokusagentur « ihre eigene Beteiligung immer nur als Impuls oder Schub, als Wegeanbahnung oder als Modellentwicklung, also exemplarisch, versteht. Dies ist auch aus Ressourcen-Sicht geboten. Schließlich geht es um die Wechselbeziehung zwischen konkreter fachlicher Problemlösung und übergreifendem Fortschritt in Richtung gelebter Vielfalt. "Migration/Integration und die Sicherung gleichberechtigter Teilhabe« ist eine dynamische gesellschaftliche Aufgabe, deren »Übersetzung« in Handeln eine erhebliche migrationsbezogene Fachkompetenz verlangt, die für die verschiedenen kommunalen Handlungsfelder anschlussfähig gemacht werden muss. Die »Fokusagentur« ist demnach zugleich als Kompetenzzentrum zu verstehen.

Eine wichtige Aufgabe besteht also in der Beschaffung, der Nutzung und Übersetzung, aber auch der Vermehrung des vorhandenen Wissens über Migration/Integration, insbesondere in Hinblick auf die lokale Ebene und die kommunale Politik (Stichwort: Wissensmanagement). Eine enge $\mathrm{Zu}$ sammenarbeit mit Migrantenorganisationen sowie mit den am Ort oder in der Region einschlägigen Forschungseinrichtungen und Hochschulen dient nicht nur dazu, das erforderliche Handlungswissen zu aktualisieren, sondern auch zur eigenen kritischen Reflexion.

\section{Idee und Berliner Wirklichkeiten}

Vom konzeptionellen Anspruch her kommt die Arbeit des Berliner Integrationsbeauftragten spätestens seit 2007 dieser Idee einer »Fokusagentur« nahe. Das vorgelegte Integrationskonzept mit seinem Monitoring-Ansatz wie auch diverse Projekte, die stets in ihrer Hebelfunktion und/oder exemplarisch gedacht sind, stehen hierfür. ${ }^{13}$

Ebenso zeigt dies die enge Verknüpfung mit dem Landesbeirat für Integrations- und Migrationsfragen. Zur Stärkung der Partizipationsmöglichkeiten von Migrantinnen und Migranten wurde im Jahr 2003 ein Landesbeirat für Integrations- und Migrationsfragen, der durch das Partizipations- und

13 Vgl. Kapitel 1. 
Integrationsgesetz (PartIntG) auf eine gesetzliche Grundlage gestellt worden ist (vgl. \$ 6 PartIntG), eingerichtet. Der Landesbeirat erarbeitet Empfehlungen zur Weiterentwicklung der Berliner Integrationspolitik. Er tagt drei- bis viermal jährlich unter dem Vorsitz der Senatorin bzw. des Senators für Integration. Mitglieder im Landesbeirat sind sieben gewählte Vertreter*innen und ihre Stellvertreter*innen von Migrant"innen- und Aussiedler*innenorganisationen, die/der Beauftragte des Berliner Senats für Integration und Migration, vom Rat der Bürgermeister der Bezirke entsandte Vertreter*innen, ein/e Vertreter/in der Bezirksbeauftragten für Integration und Migration, Verbände, Gewerkschaften und nichtstaatliche Organisationen.

Ein kommunaler Unterbau, wie im Integrationsgesetz von NRW mit den Kommunalen Integrationszentren (KI) vorgesehen und mittlerweile nahezu flächendeckend ausgebaut, ist in Berlin nur sehr rudimentär zu erkennen. Zwar sieht das Integrationsgesetz Bezirkliche Integrationsbeauftragte vor; diese sind aber institutionell schwach aufgestellt, insbesondere, weil ihnen ein >Apparat fehlt. Regelmäßige Treffen zwischen den bezirklichen Integrationsbeauftragten und dem Integrationsbeauftragten des Senats finden statt.

Faktisch bleibt die Umsetzung der Querschnittskoordinierung schwierig und letztlich unbefriedigend. Dies gilt sowohl intern für die Senatsverwaltung, der die Stelle des Integrationsbeauftragten zugeordnet ist, als auch für die Zusammenarbeit mit den anderen Senatsverwaltungen. Die strukturell schwach ausgestattete Stellung des Integrationsbeauftragten macht ihn in seinen Bemühungen zur Kooperation in hohem Maße (a) von der Kooperationsbereitschaft und Aufgeschlossenheit der jeweilig fachlich Zuständigen und (b) von der Haltung und Rückendeckung der jeweiligen Hausleitung abhängig. Die beachtlichen Erfolge der Berliner Integrationspolitik müssen vor diesem Hintergrund bewertet werden. 


\section{Verknüpfungschancen zwischen verschiedenen Politikfeldern und der Integrationspolitik}

Vor dem Hintergrund des »integrationspolitischen Neustarts« der Jahre 2007 bis 2010 bot das arbeitsmarktpolitische Programm »BerlinArbeit « ${ }^{14}$ für die Legislaturperiode 2011-2016 erhebliche Verknüpfungsmöglichkeiten. ${ }^{15}$

Dies ist an jenen zwei Handlungsfeldern des damaligen Programms »Berlin-Arbeit « gut zu erkennen, die unter integrationspolitischer Perspektive in einem engen Zusammenhang stehen, nämlich »Verstärkte Integration von Migrantinnen und Migranten in den Arbeitsmarkt « (Handlungsfeld 4) und »Fachkräftesicherung und -entwicklung« (Handlungsfeld 2). Die bei »verstärkter Integration« ins Auge gefassten Maßnahmen sind schwerpunktmäßig auf Jugendliche und junge Erwachsene mit Migrationshintergrund konzentriert und mit einem modifizierten Defizitansatz unterlegt.

Die Maßnahmenliste im Handlungsfeld »Fachkräftesicherung ...« verhält sich zu den bei »Verstärkte Integration ...« aufgeführten Maßnahmen nahezu spiegelbildlich, "mit dem schlichten, aber wichtigen Unterschied, dass Migration/Integration keine Erwähnung findet. Wir haben es also mit der schlichten Übung einer Separierung von Migration/Integration in ein gesondertes Handlungsfeld zu tun« Es »hält sich in den betrachteten Dokumenten die bisherige Sichtweise weitgehend durch, nach der es sich bei Integration/Migration um ein weiteres Aktionsfeld handele und eben nicht um eine Querschnittsaufgabe. Dies leistet immer wieder einer Tendenz zur parallelen Verdoppelung von Aktivitäten Vorschub.«

Die Etablierung eines systematischen, fokusartigen Bezugs zwischen verschiedenen Handlungsfeldern ist - so die Expertise von damals - »ein dickes Brett, das zu bohren ist«. Als Gründe werden u.a. genannt: »Sie erstrecken sich von einer vorherrschend sozialpolitisch bestimmten Definition von

14 Senatorin für Arbeit, Integration und Frauen 2012: BerlinArbeit. Strategische Neuausrichtung der Arbeitsmarkt- und Berufsbildungspolitik des Landes Berlin in der Legislaturperiode 2011 bis 2016, Eckpunkte, Berlin/BerlinArbeit. Gemeinsames Rahmen-Arbeitsmarktprogramm des Landes Berlin und der Regionaldirektion Berlin-Brandenburg der Bundesagentur für Arbeit, veröffentlicht am 30.7.2013. https://www.berlin-transfer.net/ de/wissenspool/politischer-rahmen/534-berlin-arbeit

15 Kruse, Wilfried: Gut Ankommen in der Arbeitswelt. Expertise für den Beauftragten des Berliner Senats für Integration und Migration, Unveröffentlichtes Manuskript, Dortmund 2013. 
Problemen der Arbeitsmarktintegration über die Ablehnung von Strategien positiver Diskriminierung bis hin zu Fragen von Arbeitsteilung und Kooperation innerhalb der und zwischen den Senatsverwaltungen."

Dies führt u.a. dazu, dass selbst die sachlich naheliegenden Verknüpfungen nicht hergestellt werden. Sie ergeben sich allerdings auch nicht von selbst und sind nicht vordergründig. Sie ergeben sich - und dies ist ganz explizit Aufgabe eines fachlich-politischen kooperativen Selbstaufklärungsprozesses - erst dann, „wenn man sie als Elemente einer modernisierten Integrationsstrategie im Übergang von der Schule in die Arbeitswelt versteht«. Stichworte hierzu sind z.B. eine differenzierte Betrachtung der Gruppe der Jugendlichen mit Migrationshintergrund, die Erweiterung des Verständnisses von Übergang, so dass er als erfolgreich erst dann gilt, wenn die jungen Erwachsenen gut in der Arbeitswelt angekommen sind, netzwerkförmige Unterstützungssysteme und eine verstärkte Aufmerksamkeit gegenüber institutionellen Benachteiligungen und Diskriminierungen.

Es zeigt sich an diesem Beispiel, wie schwierig es unter den gegebenen Bedingungen der 2010er Jahre war, Verknüpfungen zwischen Fach- und Integrations-/Teilhabepolitik herzustellen, sogar, wenn - wie in diesem Fall - die Handlungsfelder viele Bezüge aufweisen und die Zuständigkeiten sich in derselben Senatsverwaltung befinden. Aber es geht auch anders. Hierfür findet sich sogar im Kontext von »BerlinArbeit« ein Beispiel, nämlich die interkulturelle Öffnung von Jobcenter und Arbeitsagenturen. Wird dies auch zunächst über die Förderung »interkultureller Kompetenz« des Beratungspersonals eng geführt, ${ }^{16}$ so weist diese Vereinbarung doch in die Zukunft. Es kommt also durchaus auch auf die Initiative des Integrationsbeauftragten an, die allerdings auf Widerhall angewiesen ist.

Im Sinne einer ganzheitlichen, auf die strategischen Ziele, wie sie im Integrationskonzept von 2007 formuliert sind, ausgerichteten Integrationsund Teilhabepolitik wäre es produktiv gewesen, die sich anbietenden und sachlich gebotenen Verknüpfungen zwischen Integrations-, Arbeits- und Bildungspolitik auch tatsächlich herzustellen und in der Folge mit stabilen

16 Das wird für die Jugendberufsagenturen ebenfalls wichtig: Konzeptionell ist entscheidend, dass sich die interkulturelle Öffnung keineswegs in der höheren interkulturellen Sensibilisierung des Beratungspersonals erschöpfen darf, sondern sich auf ein gemeinsam erarbeitetes und geteiltes Verständnis von Prozessen gelingender Übergänge beziehen muss. 
Kooperationen zu hinterlegen. Dies hätte auch der anspruchsvollen Aufgabe »strategischer Steuerung« entsprochen, wie sie für das Amt des Integrationsbeauftragten 2010 gesetzlich fixiert wurde.

Im Rückblick bleibt festzustellen, dass diese Verknüpfungen wohl immer wieder einmal punktuell, aber nicht systematisch und stabil gelungen sind. Dies hat verschiedene Gründe, von denen die schon angesprochenen strukturellen Schwächen der Ausgestaltung des Amts des Integrationsbeauftragten einen darstellen.

Deshalb kommt der Sachverständigenrat deutscher Stiftungen für Integration und Migration auf der Basis einer kritischen Bestandsaufnahme der vorliegenden Integrationsgesetze u.a. zu folgender Empfehlung: »Integration als Querschnittsaufgabe erfordert wirksame und funktionierende Strukturen der Abstimmung, Umsetzung und Kontrolle. Dazu gehört eine ressortübergreifende Zusammenarbeit ebenso wie eine Zusammenarbeit zwischen Land und Kommunen sowie mit Akteuren außerhalb von Politik und Verwaltung. ${ }^{17}$

\section{Fazit}

Im Anschluss an die im ersten Kapitel skizzierte, durch die Verabschiedung des Integrationskonzepts 2007 gegebene integrationspolitische Ausgangslage wird nun der Institutionalisierung der Integrationspolitik in Berlin weiter nachgegangen. Dabei rückt die Figur des/der Integrationsbeauftragten des Senats als eine Berliner Besonderheit in den Blickpunkt.

Ob der/die Integrationsbeauftragte nämlich die dieser Funktion im Partizipations- und Integrationsgesetz von 2010 zugeschriebene anspruchsvolle Aufgabe der "strategischen Steuerung", also der Initiierung und Umsetzung einer als Querschnittsaufgabe verstandenen Integrationspolitik befriedigend umsetzen kann, hängt von der ihr zugemessenen Durchsetzungsmacht ab. Wie komplex diese Aufgabe ist, wird deutlich, wenn man - wie in diesem Kapitel diskutiert - die Funktion des Integrationsbeauftragten als

17 Sachverständigenrat deutscher Stiftungen für Integration und Migration: Papiertiger oder Meilensteine? Die Integrationsgesetze der Bundesländer im Vergleich (=Policy Brief Oktober 2017), Berlin 2017, S. 25. 
eine Agentur zur Verankerung des Fokus Migration in allen Handlungsfeldern des Senats versteht.

Die schlussendlich erfolgte Platzierung der Funktion des/der Integrationsbeauftragten des Senats als Abteilung innerhalb einer Senatsverwaltung, die auch Integration in ihrem Titel trägt, markiert die zwiespältige institutionelle Lage, in der sich der/die Integrationsbeauftragte befindet. Von daher erwies sich die sachlich gebotene Verknüpfung von Integration und Teilhabe mit den verschiedenen Fachpolitiken von Anfang an als schwierig, wie dies hier beispielhaft am Feld der integrationspolitisch gebotenen Öffnung von Berufsausbildung gezeigt wird.

Gelingende Verknüpfungen zwischen Integrations- und Teilhabepolitik und den verschiedenen Fachpolitiken bilden einen wichtigen förderlichen Rahmen für die Wirksamkeit von Leitprojekten. Die Herstellung der Verknüpfung kann den Leitprojekten aber im Grunde nicht zugemutet werden. Im Rückblick bleibt festzustellen: Die zu den komplexen und anspruchsvollen Aufgaben im Kontrast stehende strukturelle und auch personelle und ressourcenbezogene »Unterausstattung« der Funktion des/der Integrationsbeauftragten hätte nach Lage der Dinge nur durch fortdauernde hartnäckige Überzeugungsarbeit, vertrauensbildende Maßnahmen und durch Kooperationsinitiativen aus dem Amt selbst heraus einigermaßen kompensiert werden können. Diese Initiativen gab es, aber sie waren - in summa - für einen erheblichen Zeitraum nicht stark genug, um eine dauerhafte und belastbare kooperative Verknüpfungsstruktur zu etablieren

Es sind also zwei Schieflagen, die den Start der neuen Integrationspolitik mitprägen: Erstens liefen die »Programmatik«, wie sie im Integrationskonzept von 2007 zum Ausdruck kommt, und die anspruchsvolle und komplexe Aufgabenstellung für den/die Integrationsbeauftragte/n aus dem Jahr 2010 politisch der strukturellen und operativen Ausstattung der Funktion weit voraus. Und zweitens war das erneuerte Verständnis von Integration und Teilhabe als Querschnittsaufgabe zwar eine politische Vorgabe, aber deshalb noch lange nicht im Alltag der Behörden und Institutionen angekommen.

Der soziale und operationelle Durchsetzungsprozess einer erneuerten integrationspolitischen Orientierung stellte sich - eigentlich nicht verwunderlich - als langwierig und schwierig heraus. Heute sind die Voraussetzungen für eine systematische Verknüpfungspolitik wesentlich ausgereifter, und es gibt hierfür bereits tragfähige Verabredungen. Insgesamt bleibt sie 
überfällig. Darauf wird später, insbesondere in den Kapiteln 9 und 10, eingegangen.

Vor dem Hintergrund der nun etwas ausführlicher beleuchteten integrationspolitischen Rahmenbedingungen widmen sich die folgenden Kapitel dem Leitprojekt Berlin braucht dich!, das hier für sich, also mit seinen konkreten Zielen, Praktiken und Wirkungen, und zugleich auch als ein beispielhaftes Vorhaben für die Berliner Integrations- und Teilhabepolitik dieser Jahre steht. 



\section{Kapitel 3}

\section{Die Ausgangslage beim Übergang Schule - Arbeitswelt im Berlin in den 2010er Jahren}

Die Frage, wie Übergänge von der Schule in Berufsausbildung generell vermehrt und insbesondere für Jugendliche, die bisher in diesen wenig Chancen hatten, erfolgreicher gemacht werden können, erhält im Berlin um das Jahr 2010 ein erhebliches Gewicht. Das zeigt auch eine damals durchgeführte Recherche. ${ }^{1}$ Dort wird neben den Aktivitäten, die vom Berliner Integrationsbeauftragten ausgehen, vor allem auf zwei Entwicklungen hingewiesen: auf das wieder erwachte Interesse der Agentur für Arbeit am Feld »Berufsorientierung« und auf die laufende Schulreform.

Schon 2006 war die Öffentlichkeit durch einen "Brandbrief« aus der Rütli-Hauptschule in Neukölln auf die Misere insbesondere an Schulen mit einem hohen Anteil von Schüler/innen aus Familien mit Migrationsgeschichte und Armutsmilieus aufmerksam geworden. Die Debatte darüber erreichte auch den Bundestag. Im »Fall Rütli« führte dies zu einem umfassenden pädagogischen Umbau der Schule, der heute allgemein als vorbildlich betrachtet wird. ${ }^{2} 2007$ war durch die gemeinsame Initiative der Agentur für Arbeit und der Senatsverwaltungen für Bildung, Wissenschaft und Forschung und Integration, Arbeit und Soziales das Berliner Programm zur »Vertieften Berufsorientierung« (BVBO) auf den Weg gebracht worden. Dem schiebt die Regionaldirektion Berlin-Brandenburg im Dezember 2008

1 W. Kruse: Berlin braucht dich! - über den öffentlichen Dienst hinaus? Dortmund 2010.

2 Vgl. u.a.: https://www.spiegel.de/lebenundlernen/schule/ruetli-schule-in-berlin-neukoelln-vom-problemfall-zur-vorzeigeschule-a-1199251.html vom 1.4.2018 
ein Strategiepapier nach, ${ }^{3}$ in dem sie sich erneut als der zentrale Partner der Schulen für die Berufsorientierung anbietet.

In Hinblick auf die Schulreform resümiert die 2010 veröffentlichte Recherche:

Das Land Berlin befindet sich im Prozess der Umsetzung einer weitgehenden Schulreform, die auch neue Rahmenbedingungen für den Übergang von der Schule in die Arbeitswelt setzt. Insbesondere sind drei Merkmale dieses künftigen neuen Schulsystems zu nennen, die neue Kontexte schaffen: (1) Die Überwindung der bisherigen Dreigliedrigkeit des Schulsystems mit der Einführung der Sekundarschule, neben der das Gymnasium erhalten bleibt, (2) die Einführung des Ganztags, die mit der Idee verbunden ist, die Schule stärker ızum Leben hin zu öffnen, wofür Kooperationspartner erforderlich sind, und (3) die Etablierung des Dualen Lernens als Unterrichtsprinzip insbesondere für die Sekundarschule. Vom Dualen Lernen wird eine Remotivierung der Schülerinnen und Schüler und eine verbesserte Berufsorientierung erwartet. ${ }^{4}$

\section{Der Übergang von der Schule in die Arbeitswelt wird langsam zum Thema}

Diese Berliner Entwicklungen stehen nicht isoliert da. Vielmehr ist bundesweit eine Art Aufbruch in Hinblick auf eine Verbesserung der Übergänge von der Schule in die Berufsausbildung zu beobachten. Dieser ist zunächst allerdings sehr stark regional bzw. lokal akzentuiert. Er wird einerseits durch Bundesprogramme, wie »Lernende Regionen« oder »Kompetenzen fördern« gefördert, andererseits aber auch durch eine neue und deutliche Positionierung der Kommunen selbst, wie die »Aachener Erklärung« des Deutschen Städtetags von 2007 und die »Arbeitsgemeinschaft Weinheimer Initiative«

3 Regionaldirektion Berlin-Brandenburg der Bundeagentur für Arbeit: Strategische Neuorientierung der Berufsorientierung, Dokument vom 2. Dezember 2008.

4 W. Kruse: Berlin braucht dich! - über den Öffentlichen Dienst hinaus? S. 58, Berlin/ Dortmund 2010. 
mit ihrem Konzept von »Kommunaler Koordinierung und Lokaler Verantwortungsgemeinschaft.$^{5}$

Der kommunalen Positionierung geht es dabei auch um die Anerkennung der erheblichen Anstrengungen zu Bildung und Übergang, die auf lokaler Ebene unternommen werden, aber im strikten Sinne »freiwillig« sind, und einer Flankierung bedürfen. Hier kommen nun vor allem die Länder ins Spiel. Tatsächlich bewegt sich in den ersten zo10er Jahren auf Länderebene einiges im Sinne der Etablierung von landesweiten Übergangssystemen, so z.B. in Hamburg und in Nordrhein-Westfalen, aber auch mit bemerkenswerten Ansätzen in Schleswig-Holstein.

Berlin war also gewissermaßen von zwei Seiten unter Druck, nämlich im Vergleich mit anderen Bundesländern und insbesondere mit Hamburg, das als ebenfalls großer Stadtstaat öfters zum Vergleich herangezogen wird, und aufgrund der durchaus gravierenden Defizite beim Übergang von der Schule in die Ausbildung, die Berlin aufwies. Schließlich entsteht in diesen Jahren zwar kein akzeptierter und verbindlicher Gesamtentwurf, aber es wird an vielen Baustellen durchaus produktiv gearbeitet.

Einschränkend muss gesagt werden, dass Berlin mit den Bundesländern, die sich auf den Weg zu einem Übergangssystem gemacht haben, teilt, dass die duale Berufsausbildung, ihre Qualität und ihre Zugänglichkeit, weniger zum Thema gemacht wird, sondern es vor allem um die bessere Heranführung der Jugendlichen an die Ausbildung geht - und damit neben den Jugendlichen selbst vor allem die Schule im Fokus steht. Eine kurzzeitige "Ausnahme von der Regel « gab es bei Berlin braucht dich! durch eine intensive Beschäftigung mit Fragen der Ausbildungssicherung. ${ }^{6}$

In Berlin, wo ein deutlicher Mangel an Ausbildungsplätzen fortbestand und daher die ausbildenden Betriebe aus einer größeren Zahl von Bewerber*innen auswählen konnten, wurde politisch über Jahre auf die Förderung über- und außerbetrieblicher Ausbildung gesetzt, ohne dies in einem Gesamtsystem zu verknüpfen, obwohl mit den Oberstufenzentren ein innovatives Format zur Verfügung stand und steht. Dass auch in Berlin eine solche integrierte Reform-Agenda erforderlich wäre, darauf wies schon 1999

\footnotetext{
5 Vgl. www.kommunale-koordinierung.de

6 Goethe, Katharina: Erfolg in der Ausbildung. Projektergebnisse und Empfehlungen für eine bessere Ausbildungssicherung bei Auszubildenden mit Migrationshintergrund, Ber$\operatorname{lin} 2012$.
} 
ein Memorandum »Leitlinien für die Schaffung eines dualen, pluralen und modularen Systems des lebensbegleitenden Lernens « hin, ${ }^{7}$ das damals vom Beirat der Berliner Senatsverwaltung für Arbeit, Berufliche Bildung und Frauen vorgelegt wurde - ein weitgehender Reformansatz, der sich nicht auf Berufsorientierung reduziert, sondern auf die Strukturfragen des Dualen Systems selbst fokussiert.

Auch in Hinblick auf netzwerkgestützte Übergangsförderung für Jugendliche mit oder aus Familien mit Migrationsgeschichte - wie z.B. im erwähnten Bundesprogramm »Kompetenzen fördern« - stand Berlin nicht allein und konnte eine Vorreiter-Rolle allenfalls in der Hinsicht für sich reklamieren, als dieser Ansatz schon früh zum Bestandteil eines umfassenden Integrationskonzepts wurde.

\section{Berufsorientierung: Berliner Aufbruchsjahre}

Ganz zweifellos erhielt die Berufsorientierung an den Berliner Sekundarschulen durch das schrittweise flächendeckend eingeführte Programm der "Berliner Vertieften Berufsorientierung $(\mathrm{BVBO})^{8}$ einen wichtigen Schub, ebenso wie das als Unterrichtsprinzip formulierte "Duale Lernen «.

Das Landesprogramm der Vertieften Berufsorientierung startete bereits im Jahr 2007 und war darauf ausgerichtet, dass Schulen die Angebote externer Partner gezielt für die Stärkung der Berufsorientierung nutzen können. Es handelt sich um ein Förderprogramm ab Klasse 8, welches eine Schlüsselrolle von Trägern in der Zusammenarbeit zwischen Schulen und außerschulischen Lernorten vorsieht. Es wurden verschiedene Module angeboten, aus den die Schulen wählen können. Im Schuljahr 2011/2012 wurde z.B. in den Sekundarschulen das Modul »Berufspraktische Erprobungen« mit Abstand am meisten gewählt. Zwischen BVBO und Berlin braucht dich! in der NachKampagnen-Phase gibt es Gemeinsamkeiten und Unterschiede.

\footnotetext{
7 www.cedefop.europa.eu/files/etv/Upload/Information_resources/Bookshop/119/20_de_ memorandum.pdf.

8 Vgl. https://www.berlin.de/sen/arbeit/ausbildung/berufsorientierung/bvbo/

9 https://www.berlin.de/sen/bildung/schule-und-beruf/berufs-und-studienorientierung/ duales-lernen/
} 
Berlin braucht dich! unterscheidet sich von BVBO in vielerlei Hinsicht: erstens in der besonderen Aufmerksamkeit gegenüber Schüler/innen mit Migrationshintergrund, zweitens durch einen Pool von qualitativ guten betrieblichen Praktika, die den mitarbeitenden Schulen zur Verfügung gestellt werden, drittens durch den Anspruch, die stufige Abfolge von Betriebsbegegnungen ab Klasse 7 in eine systematische schulische Berufsorientierung einzubetten (Qualifizierte Vierstufigkeit) und schließlich viertens durch die verbindliche Kooperation der beteiligten Schulen und Betriebe in einem Konsortium.

Während BVBO auf eine Einbeziehung aller Sekundarschulen angelegt ist, bleibt Berlin braucht dich! aber ein modellhafter Ansatz der Kooperation zwischen Schulen und Betrieben, vor allem des Öffentlichen Dienstes und Öffentlichen Unternehmen. Es wäre von daher sinnvoll gewesen, zwischen beiden eine enge Zusammenarbeit zu vereinbaren, zumal in diversen Schulen beide anzutreffen waren. Berlin braucht dich! stellt erhebliche Anforderungen an die Schulen, während BVBO eher wie ein externer Unterstützer der Schulen bei der Bewältigung der Anforderung der Berufsorientierung funktioniert. Diese Anforderungen an die Schulen wurden durch die Einführung des »Dualen Lernens« noch einmal akzentuiert.

"Duales Lernen" ist für die Integrierten Sekundarschulen bereits in der Neufassung des Berliner Schulgesetzes vom 28. Juni 2010 verankert. Als Leitfach hierfür gilt das neu konzipierte Schulfach Wirtschaft-ArbeitTechnik (WAT), dessen Rahmenlehrplan zum Schuljahr 2012/2013 in Kraft gesetzt wurde. "Duales Lernen« umfasst Aktivitäten zur Berufs- und Studienorientierung sowie Praxisplätze an geeigneten Lernorten. Praxisplätze an geeigneten Lernorten sind zum Beispiel eigene schulische Werkstätten, Schülerfirmen, berufliche Schulen und öffentliche Verwaltungen, betriebliche Werkstätten, Betriebe und überbetriebliche oder außerbetriebliche Bildungsstätten.« Im Unterschied hierzu geht Berlin braucht dich! von der Unverzichtbarkeit des »Lernorts Betrieb« aus.

Auch diese "Handreichung" atmet im Übrigen ein Grundprinzip der Berliner Schulpolitik, nämlich die pointierte Eigenverantwortlichkeit jeder einzelnen Schule: »Jede Schule entscheidet eigenverantwortlich, welche Angebote des Dualen Lernens durchgeführt werden und legt die Angebote und deren Umfang im Schulprogramm fest." Sie ist in ihren Wirkungen zwiespältig: auf der einen Seite fördert sie die Initiativen der einzelnen Schulen, 
allerdings nur im Rahmen einer eingeschränkten Budgetverfügung, auf der anderen Seite aber macht sie jede Schule auch zu einer Art »Welt für sich«.

\section{Ideen zu einem Übergangsmanagement - und was daraus wird}

Das aus Bundesmitteln geförderte Projekt »RÜM - Regionales Übergangsmanagement « brachte bis 2012 eine Vielzahl verschiedener Akteure des Übergangs Schule-Arbeitswelt in diversen Arbeitsformen zusammen, von denen die sogenannten Schnittstellenkonferenzen strukturbildendes Potenzial hatten - aber nicht weitergeführt wurden. Denn mit »Schnittstellen« waren Überschneidungsbereiche von Handlungsfeldern der verschiedenen Akteure entlang der Übergangsverläufe bezeichnet. ${ }^{10}$ Der Rahmen des RÜM-Vorhabens machte es auch möglich, über die landesweite Steuerung des Übergangs Schule - Beruf laut nachzudenken. ${ }^{11}$

Auch das Programm »BerlinArbeit« bot verschiedene Anknüpfungspunkte für eine verstärkte Verknüpfung von Integration und Fachkräftesicherung und zur Stärkung von Übergängen, die aber weitgehend ungenutzt blieben. ${ }^{12}$

Parallel hierzu wurde auch in anderen Kontexten, wie z.B. der kiezbezogenen Jugendkulturarbeit mit Jugendlichen mit Migrationsgeschichte an innovativen Ansätzen zur Berufsorientierung gearbeitet. ${ }^{13}$ Zugleich fand eine Auseinandersetzung damit statt, wie Jugendliche mit Migrationsgeschichte zu Berufsausbildung stehen. ${ }^{14}$ Auch Arbeit und Bildung e.V. zum Beispiel ist

10 www.perspektive-berufsabschluss.de/de/207.php

11 Kruse, Wilfried 2012: »Dimensionen landesweiter Steuerung am Übergang Schule - Beruf: Was kann, muss, was sollte ein RÜM leisten?«, Vortrag vom 15.3.2012; www.perspektive-berufsabschluss.de/downloads/Downloads_Projekte_Uebergangsmanagement/ Uebergangsmanagement_Berlin_Beitrag_Wilfried_Kruse.pdf

12 Vgl. Kapitel 2.

13 JugendtheaterBüro Berlin der Initiative Grenzenlos e.V.: Berufsorientierung durch Jugendkulturarbeit. Einfluss der Arbeit im JugendtheaterBüro Berlin auf die Lebensperspektiven teilnehmender Jugendlicher 2009-2012, Berlin 2012, www.grenzen-los.eu/wpcontent/uploads/2012/05/Brosch\%C3\%BCre_JTB_Web.pdf

14 Lehmkuhl, Kirsten/Eckelt, Marcus/Schöler Cornelia: Ausbildung - eine attraktive Zukunftsoption? Schülerinnen und Schüler mit Migrationshintergrund erleben die Arbeitswelt. Expertise für BQN Berlin, Berlin 2011. 
seit langer Zeit mit verschiedenen Projekten in diesem Feld einschlägig tätig. ${ }^{15}$

\section{Ein Entwicklungsprojekt: das Landeskonzept Berufs- und Studienorientierung}

Anfang der 2010er Jahre setzte die Senatsbildungsverwaltung auch einen partizipativen Prozess der Entwicklung eines Berliner Berufsorientierungskonzepts in Gang. Es ist also diese - hier nur in groben Zügen skizzierte - Berliner Landschaft "in Bewegung «, auf die das an Fahrt gewinnende Vorhaben Berlin braucht dich! traf und in die es sich zunehmend einbrachte. Dass dies möglich war, erklärt sich vor allem daraus, dass die Verbesserung der Praktika in der Berufsorientierung »in der Luft« lag und die von Berlin braucht dich! eingenommene Position der Unverzichtbarkeit des "Lernorts Betrieb« Resonanz fand.

In der breit angelegten und mehrere Jahre andauernden Arbeit am Berufsorientierungskonzept gab er verschiedene Formate, so z.B. eine Fachgesprächsreihe zur Didaktik der Berufsorientierung, an der sich neben Wissenschaftlern der Technische Universität Berlin und Praktikern auch Berlin braucht dich! beteiligen konnten, in der es um den über eine längere biografische Periode gehenden Aufbau einer Berufsorientierung im Sinne einer Fähigkeit zur bewussten Berufswahlentscheidung ging. Als Seitenstück initiierte die Berufsbildungsabteilung der Bildungsverwaltung eine Fachgesprächsreihe 2012/2013 »Betriebspraktika als Baustein der Berliner Berufsorientierung und Berufsvorbereitung", als deren Produkt ein Dokumentations-Bogen »Hinweise zum Verlauf des SchülerInnen-Praktikums aus betrieblicher Sicht« zur Beobachtung und Einschätzung des Orientie-

15 Auf Initiative des Bundesministeriums für Arbeit und Soziales und ergänzt durch Mittel der europäischen Gemeinschaftsinitiative EQUAL wurde 2005 das Netzwerk KUMULUSPLUS gegründet, dessen Ziel die berufliche Integration von Menschen mit Einwanderungsgeschichte ist. Neben der praktischen Arbeit hat das Beratungs- und Qualifizierungsnetzwerk, das von Arbeit und Bildung e.V. von 2006 bis 2011 koordiniert und geleitet wurde, im Rahmen des Nationalen Integrationsplans auf Bundesebene erfolgreich die Aufgabe verfolgt, Grundlagen für gesetzliche Initiativen und Handlungsprogramme zu entwickeln, die das Handeln der Bundesagentur für Arbeit sowie der Träger der Grundsicherung in der Arbeit mit genannter Zielgruppe unterstützen und verbessern sollen. 
rungsfortschritts von Jugendlichen während der Praktika entstand. ${ }^{16}$ Seine »Philosophie«: Es geht keinesfalls um >Beurteilungen«, sondern darum, Anhaltspunkte dafür zu gewinnen, an welchen Punkten die Jugendlichen in ihrer Auseinandersetzung mit der eigenen beruflichen Perspektive angelangt sind und wie man sie im weiteren Orientierungs- und Entscheidungsprozess unterstützen kann. Es geht also nicht um `Eigenschaften<, sondern um beobachtbare Haltungen und Fähigkeiten.

\section{Im Fokus: Betriebspraktika}

Es waren rückblickend Jahre, in denen erheblich an der Qualität von Betriebspraktika gearbeitet wurde, so z.B. angeregt von Berlin braucht dich! bei den Berliner Wasserbetrieben schon 2010, oder die Erprobung von Vorbereitungs- und Rückmeldebögen z.B. bei der Carl-von-Ossietzky-Schule etwa im selben Zeitraum. Parallel hierzu wurde im Rahmen der Berufsschuldidaktik die Debatte über selbstgesteuertes Lernen fortgeführt. ${ }^{17}$

Schließlich nahm die »Qualifizierte Vierstufigkeit« als Konzept der ab Klasse 7 aufeinander aufbauenden und in die schulische Berufsorientierung eingebetteten Abfolge von Betriebspraktika - aus Berlin braucht dich! kommend - Eingang in das im März 2015 verabschiedete »Berliner Konzept der Ausbildungs- und Studienorientierung « wie auch in die zum Schuljahr 2015/2016 überarbeitete Version der »Berliner Vertieften Berufsorientierung«. Im Kontext des Landeskonzepts werden dann ab 2015/2016 an den Integrierten Sekundarschulen sogenannte BSO-Teams aus dem Koordinator/der Koordinatorin für Berufs- und Studienorientierung der Schule, der Berufsberatungsfachkraft der Agentur für Arbeit und einer Lehrkraft an beruflichen Schulen gebildet, die die Schüler/innen in der konkreten Anschlussgestaltung in der beruflichen Qualifizierung beraten und unterstützen sollen.

16 Kohlmeyer, Klaus/Kruse, Wilfried/Zschiesche, Tilman: Betriebspraktika als Baustein der Berliner Berufsorientierung und Berufsvorbereitung, unveröffentlichte Manuskripte, Berlin 2013.

17 Z.B. Lindemann, Hans-Jürgen (Hg.): Lern- und Arbeitsaufgaben sowie Projekt- und Arbeitsaufgaben in der Lernfeldarbeit, Berlin 2013. 


\section{Mehr Aufmerksamkeit für die beruflichen Perspektiven junger Menschen mit Migrationshintergrund}

Der Umstand, dass die Einarbeitung der »Qualifizierten Berufsorientierung« in das Landeskonzept auf Initiative des Integrationsbeauftragten und auf der Basis der Erfahrungen von Berlin braucht dich! erfolgte, sind ein Beleg dafür, dass Jugendliche mit Migrationsgeschichte als Zielgruppe und Grund für eine Verbesserung der Übergänge von der Schule in die Ausbildung nun tatsächlich landespolitisch auch in den Blick genommen worden waren.

Insgesamt findet der Ansatz von Berlin braucht dich!, die beruflichen Perspektiven von Jugendlichen mit Migrationsgeschichte zu verbessern, in diesen Jahren auch ein positives mediales Echo, ${ }^{18}$ wie auch andere Projekte und Aktivitäten, die auf eine bessere Teilhabe der Menschen mit Migrationsgeschichte zielen, z.B. das Landesrahmenprogramm $2013 \mathrm{zu}$ Integrationslotsen und Stadtteilmüttern. Zwar liegt nach Sarrazins Deutschland schafft sich ab mit Heinz Buschkowskys Neukölln ist überall nun ein direkt auf Berlin bezogenes Buch vor, in dem mit grellen Farben die Gefahr von Desintegration und Parallelgesellschaft an die Wand gemalt wird. ${ }^{19}$ Aber es bleibt bei einer positiven Grundstimmung, die auch weitere integrationspolitische Verknüpfungsinitiativen trägt. ${ }^{20}$

\section{... auch beim DGB}

Aufmerksamkeit findet das integrationspolitische Leitvorhaben Berlin braucht dich! nun auch bei Gewerkschaften, zuerst bei der IG Metall. Dass Betriebe über gute Betriebspraktika Facharbeiter-Nachwuchs auch aus dem

18 So z.B. Hyams, Judith: »Öffentlicher Dienst. Endlich angekommen. Busfahrer, Polizist, Krankenpfleger: Berlin braucht Azubis - und wirbt gezielt um Jugendliche mit Migrationshintergrund«, in: Der Tagesspiegel vom 18.4.2016.

19 Thilo Sarrazin: Deutschland schafft sich ab. Wie wir unser Land aufs Spiel setzen, München 2010; Heinz Buschkowsky: Neukölln ist überall, Berlin 2012.

20 Z.B. Dahms, Nathaly: Ausbildung und Weiterbildung als Teil der interkulturellen Personalentwicklung (=Berliner Hefte zur interkulturellen Verwaltungspraxis), Berlin 2012; oder auch: Pampel, Jana/Raschke, Christian: Die Interkulturelle Öffnung eines arbeitsmarktpolitischen Programms - am Beispiel des Landesprogramms Mentoring, Berlin 2016. 
Kreis Jugendlicher mit Migrationsgeschichte gewinnen könnten, darin sieht der erste Bevollmächtige der IG Metall den großen Vorteil des praktizierten Ansatzes, ${ }^{21}$ und befindet sich hierbei auch in Übereinstimmung mit dem Migrationsausschuss seiner Gewerkschaft.

Es gelingt ihm, den Verband der Berliner Metall- und Elektroindustrie für eine Beteiligung an Berlin braucht dich! zu gewinnen und ein gemeinsames Engagement der Sozialpartner zu initiieren. Das hat insofern über die Teilnahme an Berlin braucht dich! hinausgehende Folgen, als über dieses Engagement das gesamte Berliner Übergangssystem mit seinen strukturellen Defiziten in den Blick gerät.

Ein weitgehender Antrag zum Thema wird für die Landesbezirkskonferenz des Deutschen Gewerkschaftsbundes vorbereitet und dort im Januar 2014 beschlossen. Damit wird auch der Landesausschuss für Berufsbildung und die Sonderkommission »Ausbildungsplatzsituation und Fachkräftebedarf« des Regierenden Bürgermeisters zum Adressaten der Forderung nach einem koordinierten, transparenten und fördernden Übergangssystem. Basis dieser Forderung war die im November 2011 abgeschlossene »Vereinbarung zur Nachwuchskräftesicherung für Unternehmen durch Ausbildung«. Auch der »Masterplan Industriestadt Berlin 2010-2020« hat Fachkräftesicherung als ein wichtiges Thema.

\section{Verpasste Chancen?}

Es sind tatsächlich Jahre, in denen Berufsorientierung, Fachkräftesicherung und die berufliche Zukunft und gesellschaftliche Teilhabe insbesondere von jungen Menschen mit Migrationsgeschichte sowohl im politischen und verbandlichen Handeln als auch im öffentlichen Diskurs ein erhebliches Gewicht erhalten. Eine Übersicht aus dem Jahr 2014 zeigt die Vielzahl und das weite Spektrum der Angebote und Dienstleistungen und die differenzierte Landschaft der Akteure. ${ }^{22}$ In diesen Zusammenhang gehört auch, dass die Sonderkommission beim Regierenden Bürgermeister am 28. April 2014 die

21 Berufsbildung aktuell: »Rekrutierungs-Pipeline in die Schulen verlegen. BBaktuell im Interview mit Arno Hager«, in: Berufsbildung aktuell 2/2014, S. 3.

22 Der Beauftragte des Senats von Berlin für Integration und Migration: Integration und Migration - Ein Wegweiser für Berlin. Ausgabe 2014, Berlin 2014. 
Berliner Erklärung zum Aufbau von Jugendberufsagenturen beschließt. ${ }^{23} \mathrm{Zu}$ einer umfassenden Neugestaltung des Berliner Übergangssystems kommt es allerdings genauso wenig wie zu einer über punktuelle Erfolge hinausgehenden systematischen Verknüpfung von Integrations- und Übergangs- und Berufsbildungspolitik. ${ }^{24}$ Insofern kann man auch von einer Periode verpasster Chancen sprechen.

\section{Fazit}

Für das Thema Übergang Schule - Arbeitswelt sind die beginnenden 2010er Jahre in Berlin eine Phase des Aufbruchs. Mit den spektakulären Protestaktionen junger Geflüchteter zwischen 2012 und 2014 deutet sich an, dass sich die Frage des Umgangs mit ihnen auf der politischen Agenda vor die weitere Arbeit an der Gestaltung des Übergangs Schule-Arbeitswelt schieben wird. Vor diesem Hintergrund war es eine wichtige Entscheidung, im Jahr 2015 nach dem »Langen Sommer des Willkommens « Berlin braucht dich! nicht für Aktivitäten mit Geflüchteten einzuspannen, sondern Kontinuität zu sichern.

Den Aufbruch macht eine Vielzahl von Aktivitäten aus, die sich direkt oder indirekt auf eine Verbesserung der Übergänge von der Schule in die Arbeitswelt beziehen. Mit Blick auf den Übergang Schule - Arbeitswelt als "System « bietet die durch praktische Erprobungsschritte gestützte Konzeptarbeit des aus Bundesmitteln geförderten Projekts »RÜM-Regionales Übergangsmanagement« die weitreichendsten Ansätze. Das gilt insbesondere für die Idee von Schnittstellenkonferenzen, als einer operativen Form von Querschnittskoordinierung. Umgesetzt wurden diese Konzepte allerdings kaum. Sie bleiben weitgehend Papier.

Dagegen erfolgt tatsächlich - wenn auch mit erheblichen Defiziten, wie im Rückblick deutlich wird - die Umsetzung der Berliner Schulreform mit der Einführung der Integrierten Sekundarschule, des Ganztags, der Etablierung des Dualen Lernens als Unterrichtsprinzip vor allem für die Sekun-

$23 \mathrm{Zu}$ Jugendberufsagenturen vgl. https://www.berlin.de/sen/arbeit/ausbildung/jugendberufsagentur/

24 Zur Cesamtentwicklung in diesen Jahren vgl. Kruse, Wilfried: Das »System«der Betriebsbegegnungen von Berlin braucht dich!, seine Ausweitung auf die Metall- und Elektroindustrie und seine Einbettung in die Berliner Vertiefte Berufsorientierung. Expertise, unveröffentlichtes Manuskript 2012. 
darschulen und die Fortführung und Verbesserung der Berliner Vertieften Berufsorientierung. In diese Linie gehört, dass in kooperativer Form ein Landeskonzept Berufs- und Studienorientierung entwickelt wurde, das zum Schuljahr 2015/2016 verbindlich wird.

Es ist also nicht eine umfassendere Ausgestaltung des Übergangssystems, die in diesen Jahren in Berlin wirklich vorankommt, sondern es ist die Berufsorientierung, die im Zentrum derjenigen Aktivitäten steht, die tatsächlich Platz greifen. Eine nahezu ausschließliche Konzentration auf Berufsorientierung steht aber immer in der Gefahr, bewusst oder unbewusst Annahmen von besonders gravierenden Defiziten bei jeweils spezifischen Gruppen von Schüler*innen zu folgen. Ohne diese Problematik aus dem Blick zu verlieren, kann man für diese Jahre geradezu von einem Schub der Verbreiterung und Verbesserung von Berufsorientierung an den Berliner Schulen - und insbesondere auch an den Integrierten Sekundarschulen - sprechen.

Integrationspolitisch bleibt auch in diesen Jahren die nicht überwundene Schwierigkeit, als Querschnittsorientierung oder als »Fokus« wirksam zu werden; das gilt sowohl für den Bildungsbereich als auch für die Vorhaben im Feld von BerlinArbeit und für die entstehenden Jugendberufsagenturen, wo nur punktuell Kooperationen entstehen. Anders verhält es sich beim Landeskonzept Berufs- und Studienorientierung, dessen Erarbeitung wie eine Plattform funktioniert, und auf diese Weise auch dem integrationspolitischen Leitprojekt Berlin braucht dich! Raum gibt.

Der Beitrag von Berlin braucht dich! besteht vor allem darin, dass die Figur der "Qualifizierten Vierstufigkeit« in das Landeskonzept aufgenommen wird. Diese meint eine Abfolge von gut gestalteten Betriebsbegegnungen/ Praktika ab Klasse 7 in jeweils für die einzelne Klassenstufe angepassten Formaten. Die Qualifizierte Vierstufigkeit war in den vergangenen Jahren in Zusammenarbeit von BQN, dem beauftragten Träger, und den in Berlin braucht dich! mitarbeitenden Betrieben und Schulen entwickelt und erprobt worden. Das Konzept war von zwei damaligen Grundüberzeugungen getragen: nämlich der Notwendigkeit der frühen, schrittweise aufbauenden und sich erweiternden Heranführung an Arbeitswelt und Ausbildung und des dafür als unverzichtbar erachteten Lernorts Betrieb.

Mit der Konzentration auf Berufsorientierung als »Unterbau« für die weitere Öffnung von Ausbildung entstehen beispielhafte Gestaltungsansätze guter Betriebsbegegnungen, Leitfäden und Handreichungen. Es werden 
Betriebsbegegnungen in immer größerer Zahl organisiert. Zugleich entsteht aus der Konzentration auf Berufsorientierung ein Ungleichgewicht. Da Berufsorientierung in der Schule geschieht, muss sie in entsprechende schulische Konzepte und Prozesse sorgfältig eingebettet sein. So stehen die Schulen, die im mittlerweile entstandenen Konsortium mitwirken, stärker im Fokus als die Betriebe, deren Beitrag im Wesentlichen in der Bereitstellung von Plätzen für Betriebsbegegnungen besteht. Hingegen rückt die Sicherung tatsächlicher Zugänge zur Ausbildung und des Ausbildungserfolgs, für die die Betriebe vorrangig verantwortlich sind, aus dem Blick. Sie werden von der Anforderung entlastet, ihre Ausbildung vielfaltorientiert auszurichten. Die Fixierung auf Berufsorientierung wird durch die Zusammensetzung des Konsortiums noch verstärkt. Im folgenden Kapitel wird dies genauer betrachtet. 



\section{Kapitel 4}

\section{Ein Konsortium entsteht}

\section{Die Anfänge: Eine Kampagne öffentlicher Arbeitgeber}

Bei Berlin braucht dich! als im engeren Sinne integrationspolitisches Vorhaben, wie es sich seit 2006 entwickelt hat, handelte es sich im Kern um eine Kampagne, wenn man so will, sogar um eine Art politisch initiierter Werbekampagne. Vor dem Hintergrund des geringen Anteils von Jugendlichen aus Familien mit Migrationsgeschichte war entschieden worden, den Öffentlichen Dienst und - später - die Betriebe mit Landesbeteiligung in eine Vorreiter-Rolle zu bringen.

Der Slogan »Berlin braucht dich!« wurde mit einer Aufforderung verbunden: »Bewirb Dich jetzt beim Land Berlin!«Die Plakate dieser Kampagne zeigten junge Leute, die unschwer als solche "mit Migrationshintergrund « zu erkennen waren. ${ }^{1}$ Auf diese Weise sollte die Botschaft an diese Zielgruppe gehen und sie zu Bewerbungen motivieren. In Konferenzen und Auftritten erklärten öffentliche Arbeitgeber, die entweder diese Ziele teilten oder für die nächsten Jahre vielleicht auch einen Rückgang von Bewerbungen befürchteten, sich vor allem aber in die Pflicht genommen fühlten, ihre Offenheit gegenüber Bewerbungen aus diesem Personenkreis heraus.

1 Wie schwierig solche Zuschreibungen sind, wurde in einem Gespräch mit den Schüler*innen deutlich, die auf dem ersten Plakat der Kampagne abgebildet sind, S. 34. Die Laterne und die Bilder der Schüler*innen sollten Vielfalt anzeigen. Die Schüler*innen selbst erkannten nichts »Fremdes« an dem Motiv: »Das sind Berliner/-innen« wie wir, sagten sie. Das Plakat erreichte gleichwohl das selbstgesetzte Ziel. Viele neue Auszubildende schon des ersten Jahres sagten, dass sie ohne eine so direkte Ansprache nicht damit gerechnet hätten, dass der Öffentliche Dienst Kids wie uns will. 
Es handelte sich also im Kern, wie der damalige Integrationsbeauftragte Piening mehrfach unterstrich, um eine »Arbeitgeber-Kampagne«. Anfänglich kam es über die Teilnahme an dieser Kampagne hinaus zu keiner engeren Zusammenarbeit. Insofern war die Kampagne zunächst eher eine Art »öffentlicher Paukenschlag" mit durchaus erheblichen medialen Wirkungen und unterschied sich in ihrem punktuellen und demonstrativen Charakter von der Arbeitsweise, die das BQN-Projekt im Bundesprogramm "Kompetenzen fördern « bereits entwickelt hatte. ${ }^{2}$ Wohl aber verband beide das Ziel, die duale Ausbildung für Jugendliche mit Migrationsgeschichte stärker als bisher zu öffnen. Dabei bestand die Überzeugung, dass dies nicht nur die Bereitschaft von Arbeitgebern voraussetzen würde, sondern auch eine wesentlich verbesserte Berufsorientierung.

\section{Bereitstellung von Betriebsbegegnungen: Initial für die Entstehung des Konsortiums}

Bei ihrem Start war die Kampagne noch nicht mit dem Ansatz verbesserter Berufsorientierung verbunden. Bald entstand aber die Idee, die an der Kampagne beteiligten Betriebe für die gezielte Bereitstellung von Praktika zu gewinnen, die bewusst als Betriebsbegegnungen bezeichnet wurden, um ihren wechselseitigen Charakter hervorzuheben. Dies geschah in gemeinsamen Veranstaltungen, um zu erreichen, dass ein solcher Ansatz von möglichst vielen Kampagnen-Betrieben gemeinsam getragen, gestaltet und verantwortet wurde. Aus den Papieren aus diesen Jahren wird deutlich, dass Betriebspraktika, die in den vier letzten Schuljahren aufeinander folgen sollten, als ein wichtiger oder sogar unverzichtbarer Unterbau für die Kampagne gedacht waren.

Mit den ersten positiven Reaktionen seitens der Betriebe und Verwaltungen wurde schon bald der Grundstein für eine Art Konsortium gelegt. Der Begriff »Konsortium« kommt aus der Wirtschaftswelt und meint dort ein zeitlich befristetes Zusammengehen verschiedener Unternehmen, z.B. als Bietergemeinschaft. Aber auch in der Projektförderung wird der Begriff »Konsortium« verwendet. Gemeinsam ist der Definition, dass es sich um einen zeitlich befristeten Zusammenschluss von gleichberechtigten juristi-

2 Vgl. Kapitel 1. 
schen Personen handelt, mit dem Ziel, ein Vorhaben gemeinsam durchzuführen.

Konsortien sind also projektorientierte Organisationen, die sich selbst Regeln ihrer Außenvertretung und ihrer internen Prozeduren geben. Betriebe, die sich zu einem Projekt zusammentun, betriebliche Praktika weiterzuentwickeln, um auch Jugendliche mit Migrationshintergrund verstärkt für die Ausbildung zu gewinnen, könnte man also als ein Konsortium bezeichnen. Und zwar vor allem dann, wenn die Konsortialmitglieder selbst verantwortlich und gemeinsam für die Zielerreichung tätig werden.

Diese Idee von reinen Arbeitgeber-Konsortien spielte im Übrigen in der Anfangszeit von Berlin braucht dich! durchaus eine Rolle, z.B. auch bei Überlegungen, den sich abzeichnenden Doppelimpuls von Kampagne und Praktika nacheinander in verschiedenen Branchen zu platzieren und die weiteren Aktivitäten zur Öffnung von Berufsausbildung den jeweiligen BranchenKonsortien zu überlassen. Mit einigen solcher Branchen, in denen bereits die Sorge um Fachkräftesicherung wuchs, gab es in diesem Sinne damals Vorgespräche.

Auch beim Beitritt der Metall- und Elektroindustrie (M\&E) zu Berlin braucht dich!, der 2013 vor allem durch eine Initiative der IG Metall erfolgte, handelte es sich um eine Art Branchen-Konsortium, mit dem wichtigen Unterschied, dass die Sozialpartner hier von vornherein gemeinsam als Motoren wirkten. Die beteiligten M\&E-Betriebe bilden auch heute noch gewissermaßen eine »Branchengruppe«.

\section{Die etwas andere Konsortial-Idee: Betriebe und Schulen gemeinsam}

Dieser betriebszentrierte Weg, der durch den Kampagnen-Einstieg nahelegt war, wurde aber nicht weiter beschritten, sondern entscheidend modifiziert, nämlich durch die Erweiterung der Partnerschaft um mehr als 20 Sekundarschulen, die sich um eine Mitarbeit in Berlin braucht dich! bewerben mussten und einen hohen Anteil von Schüler*innen aus Familien mit Einwanderungsgeschichte aufweisen sollten. Hierfür war insbesondere auch seitens der Zuständigen im Büro des Integrationsbeauftragten optiert worden.

Für diese Erweiterung, also die aktive Einbeziehung der Sekundarschulen, gab es eine Reihe von Gründen: (1) Es ergab keinen Sinn, Betriebsbegegnungen 
auch im pädagogischen Sinne zu konzipieren, ohne zu wissen und zu erfahren, wie die Jugendlichen, um die es geht, heute »ticken«. (2) Veränderte Betriebsbegegnungen mussten erprobt werden; es lag nahe, dies in Kooperation mit Sekundarschulen durchzuführen. (3) Betriebsbegegnungen sind "punktuelle Erfahrungsschübe«, die ihre orientierende Wirkung nur dann entfalten können, wenn sie in der Schule vor- und nachbereitet werden. (4) Auch die im Hintergrund schon vorhandene Überzeugung, dass dem Risiko, dass Jugendliche mit Migrationshintergrund bei eigener Praktikumssuche bei wenig förderlichen Plätzen »hängenbleiben«, sprach für eine enge und dauerhafte Kooperation zwischen einer Gruppe von Betrieben und einer Gruppe von Schulen.

Damit bestand und besteht das Konsortium aus zwei großen Gruppen: nämlich den Betrieben und Verwaltungen auf der einen Seite und den Sekundarschulen auf der anderen Seite. Es handelte sich also um ein Konsortium neuer Art, weil die Konsortialmitglieder abstrakt dieselben Ziele $\mathrm{zu}$ verfolgen schienen (»Öffnung der Ausbildung für Jugendliche mit Migrationsgeschichte«), es konkret aber unterschiedliche Interessen gab. Diese Diskrepanz gründete sich - wie im Laufe der Zeit immer deutlicher wurde - auf einer strukturellen Schieflage, nämlich dem Umstand, dass die Schüler*innen dieser Schulen aus der Sicht der beteiligten Betriebe für deren eigene Ausbildung weniger infrage kamen. ${ }^{3}$

\section{Wechselseitige Lerneffekte}

Diese Diskrepanzen, die später immer deutlicher wurden und dazu zwangen, nach neuen Ansätzen zu suchen, spielten aber zu Beginn der gemeinsamen Aktivitäten, die sich auf die Ausgestaltung der Betriebspraktika und ihre Verankerung im schulischen Alltag richteten, kaum eine Rolle. Vielmehr entstand ein lebendiger und wechselseitig sehr interessierter Erfahrungsaustausch, denn tatsächlich - so die einhellige Wahrnehmung auf beiden Seiten - begegneten sich zwei einander ziemlich fremde Welten. Die wechselseitigen "Aha«- und Lerneffekte waren hoch und trugen eine konstruktive Zusammenarbeit in Workshops und Arbeitsgruppen, als deren Ergebnisse die verschiedenen Leitfäden zur Gestaltung der Praktika, aber auch das Konzept der »Qualifizierten Vierstufigkeit« zu betrachten ist. Die Rolle von

3 Vgl. hierzu insbesondere die Kapitel 5 und 8. 
$\mathrm{BQN}$ bestand in dieser Zeit vor allem darin, diese Zusammenarbeit - auch von eigener Neugierde getrieben - zu unterstützen, fachlich zu flankieren, zu dokumentieren und in Arbeitsmaterialien zu übersetzen.

Diese wechselseitig an der jeweiligen Arbeit, ihren Herausforderungen und Bedingungen interessierte Kooperation bildete allmählich eine solide Basis von Verständnis, Vertrauen und Verständigungsbereitschaft, von dem Berlin braucht dich! auch heute noch profitiert.

\section{Elemente von Selbststeuerung}

Aus Gründen der Nachhaltigkeit und innovativer Kontinuität, vielleicht im Sinne eines "Konsortiums als Innovationsplattform «, ging es nun auch darum zu klären, wie das Konsortium stärker zum Subjekt der Weiterentwicklung von Berlin braucht dich! werden kann und damit selbst Steuerungs- und Koordinierungsaufgaben übernimmt, die bislang - im gewissen Sinne stellvertretend - im Auftrag des Integrationsbeauftragten der Träger BQN übernommen hatte. Folglich wurde eine Art Gremien-Set entworfen und erprobt: ein Koordinierungskreis auf der Arbeitsebene und eine übergreifende Steuerungsgruppe, die besser als »Kuratorium« hätte bezeichnet werden sollen.

\section{Steuerungsgruppe}

Eine breite Zusammensetzung der Steuerungsgruppe für den Öffentlichen Dienst war angedacht worden, ${ }^{4}$ um das integrationspolitische Vorhaben zur Öffnung der Berufsausbildung in einen breiteren politisch-fachlichen Kontext platzieren. Dem Integrationsbeauftragten, der seine Aufgabe darin sah und sieht, den Fokus "Migration, Integration, Teilhabe mit den verschiedenen Fachpolitiken und gesellschaftlichen Handlungsfeldern in Beziehung zu bringen, war klar, dass Verbreitung und Dauerhaftigkeit der Öffnung von Ausbildung von einer positiven Aufnahme durch die verschie-

4 Die Steuerungsgruppe setzte sich aus Vertreter*innen folgender Gruppen zusammen: Abteilungen unterschiedlicher Senatsverwaltungen, Kammern und Gewerkschaften, Migrantenorganisationen, Wissenschaftliche Begleitung, Träger, die ebenfalls Angebote zur interkulturellen Öffnung des Übergangs in die Ausbildung vorhielten. 
denen einschlägigen Akteure abhängig sind. Da Vorhaben wie Berlin braucht dich!, auch wenn sie, wie in diesem Fall, gut ausgestattet sind, immer nur ausschnitthaft agieren und begrenzte Wirkungen erzielen können, mussten weitere Akteure dafür gewonnen werden, durch Unterstützung Verstärkereffekte zu erzielen.

Mit dem Zugehen auf Schulen und der Gestaltung von Betriebsbegegnungen als wichtige Bausteine der schulisch verantworteten Berufsorientierung z.B. war die Zusammenarbeit mit der Senatsverwaltung für Bildung aufgerufen; Verstärkereffekte konnten auch dann erwartet werden, wenn es zu Synergien mit Maßnahmen der Abteilung Arbeit kommen würde, das Ziel einer Ermunterung von Jugendlichen aus Familien mit Migrationsgeschichte bedurfte außerdem Flankierungen aus den migrantischen Communities heraus.

\section{Koordinierungskreis}

Während die Steuerungsgruppe von ihrer Zusammensetzung her eher den Charakter eines Beirats hatte, ist das Koordinierungsgremium nahe am operativen Geschäft. Es besteht aus Vertreter*innen der beteiligten Ämter und Betriebe, aus den beteiligten Schulen und aus dem Team von BQN.

"Selbstverständnis und Aufgabenwahrnehmung des Koordinierungsgremiums Öffentlicher Dienst haben im Laufe der Jahre eine bemerkenswerte Entwicklung erfahren «, heißt es im Tätigkeitsbericht der Wissenschaftlichen Begleitung aus dem Jahr 2013 $3^{5}$. ॥Immer stärker wird die enge Zusammenarbeit zwischen Betrieben und Schulen im Sinne eines faktischen Konsortiums verstanden, das kontinuierlich und zunehmend eigenverantwortlich wirkt. Konsortium - also die freiwillige, enge, verbindliche und dauerhafte Kooperation zwischen einer Gruppe von Betrieben und einer Gruppe von Schulen - war im Übrigen auch der attraktive Anknüpfungspunkt für die Sozialpartner aus dem M\&E-Bereich, so dass dies auch die Entwicklungsperspektive dort markiert.«

Für den später hinzugekommenen Metall- und Elektrosektor wurde ebenfalls eine Art Steuerungsgruppe gegründet (und damit auch ihre Sonderrolle fortgeschrieben): Die Zusammensetzung des Steuerkreises MEE spiegelte, dass

5 Kruse, Wilfried: Bericht über die Tätigkeit der Wissenschaftlichen Begleitung 2013, unveröffentlichtes Manuskript, Berlin/Dortmund, Februar 2014. 
in der Startphase begleitende Beratung und operatives Handeln noch eng zusammenliegen. Neben den einschlägigen Abteilungen für Integration, Arbeit und Bildung waren dort mit dem Unternehmensverband M\&E und der IG Metall die Sozialpartner und mit den Leitungen der Überbetrieblichen Ausbildungszentren von $\mathrm{ABB}$ und Siemens auch gewichtige Praxispartner vertreten.

\section{Zusammenwirken der Gremien}

Eine der zu lösenden Fragen war, wie die beiden Gremien, nämlich Koordinierungskreis und Steuerungsgruppe, aufeinander zu beziehen sind. Wenige Jahre lang - bis 2015 - wurde hierfür ein sehr interessantes Verfahren erprobt, nämlich eine Art interner Evaluierung. Der Koordinierungskreis erarbeitete mit Unterstützung von $\mathrm{BQN}$ und wissenschaftlicher Begleitung einen Jahresbericht, der Hinweise und Empfehlungen für die weitere Arbeit umfasste, und der Steuerungsgruppe vorgelegt wurde.

Hierzu vermerkt der schon zitierte Tätigkeitsbericht aus dem Jahr 2013: "Aus diesem Selbstverständnis des Koordinierungsgremiums ÖD, das sich als Ausdruck des konsortialen Ansatzes versteht, folgt auch, dass die namentlich genannten Mitglieder des Gremiums als Herausgeber*innen des jeweiligen Jahresberichts fungieren. Der Jahresbericht wird von BQN vorbereitet, in verschiedenen Entwurfsstadien mit dem Koordinierungsgremium erörtert, schließlich von ihm verabschiedet und zum einen an die Beauftragte des Senats für Integration und Migration und an die Steuerungsgruppe adressiert. Adressat sind aber zugleich auch alle Konsortialmitglieder, das jährliche Konsortialtreffen ist in der Regel der Ort der Vorstellung und Debatte wichtiger Ergebnisse und Empfehlungen des Jahresberichts. In allen Stadien der Erstellung und Erörterung des Jahresberichts ist die wissenschaftliche Begleitung aktiv beteiligt. « ${ }^{6}$ Dass die aus der Praxis gewonnenen Empfehlungen des Koordinierungsgremiums Öffentlicher Dienst durchaus Brisanz hatten, zeigt die folgende Passage aus den Empfehlungen von 2013, die im Frühjahr des Jahres formuliert wurden: »Das Berlin braucht dich! Modell setzt sich von der bislang üblichen Praxis ab, nach der sich jede einzelne Schule und jeder einzelne Betrieb sauf eigene Rechnung im Feld der Betriebs-

6 Kruse, Wilfried: Bericht über die Tätigkeit der wissenschaftlichen Beratung im Jahr 2013, unveröffentlichtes Manuskript, Berlin/Dortmund, Februar 2014, S. $13 f$. 
begegnungen/Praktika engagiert. Ob es gelingt, Berlin braucht dich! wirksam im Schulalltag zu verankern, hängt erheblich davon ab, wie zwischen der Senatsschulverwaltung und den beteiligten Schulen eine erfolgreiche 'Koexistenz von Berlin braucht dich! im Ensemble aller Aktivitäten im Rahmen des >Dualen Lernens، gesichert werden kann.«

\section{Konsortialtreffen als Klammer}

Die im jährlichen Abstand durchgeführten Konsortialtreffen können als eine wichtige Klammer für den Zusammenhalt des Konsortiums betrachtet werden; im Juni 2018 fand das 9. Konsortialtreffen statt. Diese Konsortialtreffen folgen der Idee einer zyklischen Arbeitsweise, in der regelmäßig eine zielorientierte Bilanz des vergangenen Jahres gezogen und Arbeitsschwerpunkte für das folgende Jahr erörtert werden.

Zugleich waren und sind die Konsortialtreffen Forum für die politische Bekräftigung und Flankierung des Vorhabens, in der Regel durch eine Rede der zuständigen Senatorin oder einer/eines ihrer Staatssekretär*innen. Sie waren und sind auch »Schaufenster « zur fachlich-politischen Öffentlichkeit und das herausgehobene jährliche Treffen der aktiv im Konsortium Mitwirkenden und insofern auch eine Bestätigung von Zugehörigkeit.

Mit den Konsortialtagungen wird auch versucht, Impulse für die Weiterentwicklung zu setzen. Das kann man an den jeweiligen Motti der Tagungen ablesen: das erste Konsortialtreffen 2010 hatte das Motto "Integration in duale Ausbildung fördern«, das zweite im darauffolgenden Jahr »Vom Modell zum Alltag«. Das dritte Motto im Jahr 2012 greift mit »Mitten im Transfer« das Hinzukommen des M\&E-Sektors auf, beim vierten Treffen im Jahr 2013 geht es um "Erfolge sichern als Herausforderung«, 2014 heißt das Motto »Fokus Migration« und das sechste Konsortialtreffen im Jahr 2015 läuft unter der Überschrift »Gut ankommen in der Ausbildung«.

\section{Koordinierung: Mehr Routine als Impulse}

Nach den ersten Jahren, in denen sich die Gremien trafen - mit insbesondere im Steuerkreis schwierigen Diskussionen, die vor allem um die Abgrenzung von Kompetenzen kreisten -, unterblieben die Sitzungen sowohl des Steuer- 
kreises ÖD als auch des Steuerkreises M\&E. Damit entfielen definierte Orte des Gesprächs mit den anderen Senatsverwaltungen und -abteilungen. Diese müssen nun anlassbezogen immer wieder neu gesucht werden. Es entfällt auch der institutionell geregelte Austausch mit Vertretern von Migrantenund Migrantinnenorganisationen. Dabei handelt es sich um ein Defizit, das bisher nicht repariert worden ist.

Lediglich das Koordinierungsgremium ÖD setzte seine Treffen fort, die aber immer mehr zu einer Art routinierter Pflichtübung wurden. Das hatte verschiedene Gründe. Zum einen fehlte mit dem Fortfall des Steuerungskreises ein wichtiger Adressat für Empfehlungen. Zum anderen repräsentierte das Koordinierungsgremium in seiner personellen Zusammensetzung die Anfangsphase von Berlin braucht dich!; die Diskussionen blieben mehr oder weniger konservativ bei der Sicherung von Praktika hängen. Es entstanden kaum Impulse zur Weiterentwicklung. Diese wurden aber auch nicht eingefordert. Die Frage, ob und wie die beteiligten Betriebe und Schulen Personen in das Gremium entsenden, also sie beauftragen, wurde im Konsortium nicht erörtert. Unscharf blieb also, ob die im Koordinierungsgremium versammelten Personen für ihre jeweiligen Organisationen oder, zwar kompetent, aber für sich selbst sprechen. Beibehalten wurde allerdings, im Vorfeld der Konsortialtagungen die Tagesordnung mit dem Koordinierungsgremium durchzusprechen.

Genauso wenig wurde erörtert, wie eine stärkere Übernahme von Steuerungsverantwortung einschließlich der damit verbundenen Regelungen und Vereinbarungen erreicht werden kann. Nach den ersten Jahren einer gewissen Aufbruchsstimmung kann nun von einem faktischen Zurückfahren bei der Selbststeuerung des Konsortiums gesprochen werden - mit der Konsequenz einer erstarkenden Dienstleistungsmentalität, die sich vor allem auf den Träger $B Q N$ richtete.

\section{Zugehörigkeit: Eine Bindung mit Langzeitwirkung}

Im Verlauf der Jahre ist dennoch bei vielen der Konsortialpartner ein zwar informelles, aber dennoch starkes Gefühl der Zugehörigkeit entstanden. Dieses Gefühl der Zugehörigkeit, das etwas anderes ist als Verbindlichkeit und Selbstaktivierung, mag sehr viel mit einzelnen handelnden Personen zu tun haben, strahlt aber auf deren jeweilige Organisationen ab. Das ist be- 
merkenswert, wenn man bedenkt, dass weder Betriebe noch Schulen aus dem Vorhaben eine direkte Förderung erhalten.

Woran liegt es also, dass eine solche Zugehörigkeit entstehen konnte und mit Schwankungen und den Ausnahmen, die die Regel bestätigen, bis heute erhalten geblieben ist? Die direkten Vorteile, die Schulen und Betriebe aus den Praktika ziehen, sind quantitativ zu gering, um dies zu erzeugen. Die Vermutung geht daher stark dahin, dass der Grund tatsächlich im »spirit« von Berlin braucht dich! liegt, ein »spirit«, der vom Integrationsbeauftragten und von BQN - und oft auch von der zuständigen politischen Spitze - angeregt und gepflegt wird. Es ist eine Art einwanderungsgesellschaftlicher "spirit«, eine Teilhabe-Philosophie, die viele Beteiligte teilen oder der sie sich zumindest nicht einfach entziehen können, auch wenn die damit verbundenen Anforderungen an die Weiterentwicklung der eigenen Organisationen oftmals als Zumutung empfunden werden, denen man auszuweichen versucht.

Abbildung 3: Plakat zu »Ausbildung, eine Frage der Einstellung", Senatsverwaltung für Inneres und Sport

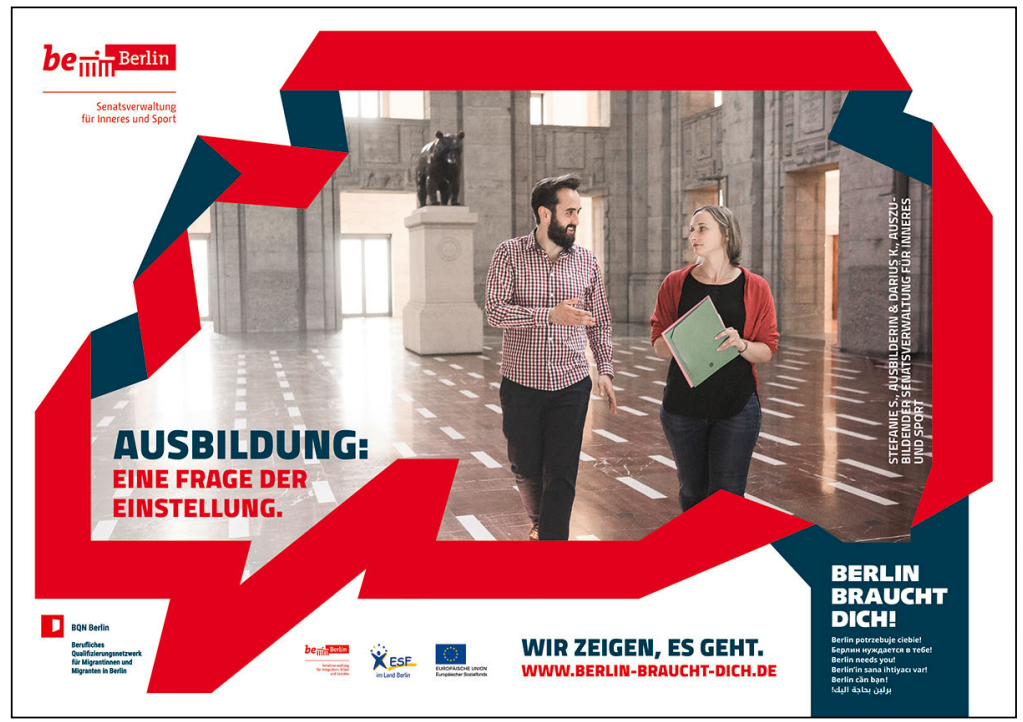

Copyright: BQN Berlin/Mikael Oskarsson 
Dass diese Basis einer geteilten Zugehörigkeit nach wie vor belastbar ist, wird nicht nur an dem Zuspruch, abgelesen an den hohen Teilnehmerzahlen, deutlich, den die Konsortialtreffen jeweils finden, sondern auch 2018 noch am Interesse einer ganzen Anzahl von Betrieben, sich bei der neuen Kampagne "Ausbildung - eine Frage der Einstellung « mit eigenen Plakaten zu beteiligen. ${ }^{7}$

Das Konsortium bleibt - wenn auch in differenzierter Weise - ansprechbar für Erfordernisse der qualitativen und quantitativen Weiterentwicklung. ${ }^{8}$ Ein markantes Beispiel hierfür ist die Gesprächsbereitschaft von Betrieben gegenüber dem Integrationsbeauftragten und dem Träger BQN, als es um die Initiierung von »Neuen Wegen in die Ausbildung«, konkret den Verzicht auf die konventionellen Einstellungsverfahren bei erfolgreich absolvierten Praktika, ging. Die bilateralen Gespräche waren durch die Bank konstruktiv und führten zu einer Reihe tragfähiger Vereinbarungen. Auf dieser Grundlage konnte in einem Treffen zwischen Senatorin Breitenbach und Geschäftsführer*innen der beteiligten Betriebe am 7. März 2018 im Roten Rathaus festgestellt werden, dass 16 Betriebe pro Ausbildungsjahr rund 50 Ausbildungsplätze bereitstellen, damit mehr Jugendliche aus Familien mit Migrationshintergrund Zugang zu qualifizierter Ausbildung erhalten. 15 Jugendliche konnten über das Pilotprojekt `Erprobung neuer Zugänge in Ausbildung‘ im September 2017 eine Ausbildung in einem Landesunternehmen beginnen.

Ein weiterer wichtiger Hinweis auf die Lebenskraft des Konsortiums ist auch, dass sich die Sozialpartner von M\&E regelmäßig mit BQN treffen, um die Reflexion über die gemeinsame Arbeit fortzuführen und neue Impulse zu setzen.

Auch die Gespräche mit Sekundarschulen, die sich über entsprechende zielorientierte Kooperationsvereinbaren in besonderer Weise für eine Erhöhung des Zugangs zu Ausbildung engagieren wollen, gehen voran. BQN sorgt flankierend dafür, dass mit Formaten wie »Partnertreffen« und »Planungsworkshops « das Potenzial des Konsortiums als Innovationsplattform erhalten bleibt. Dass dies auch nach zehn Jahren nicht selbstverständlich ist, belegen die Erfahrungen aus dem BQN-Team. Das Konsortium ist immer noch unfertig.

\footnotetext{
7 Kampagne »Ausbildung - Eine Frage der Einstellung« - Namhafte Hauptstadtbetriebe stärken Ausbildung als Integrationskraft, Pressemitteilung des Integrationsbeauftragten vom 30.11.2017, https://www.berlin.de/lb/intmig/service/pressemitteilungen/2017/pressemitteilung.653755.php

$8 \mathrm{Vgl}$. hierzu und zu den weiteren Beispielen das Kapitel 8.
} 
Abbildung 4-7: Plakate zu "Ausbildung, eine Frage der Einstellung", Bezirksamt Neukölln, Vivantes, Berliner Wasserbetriebe, Berliner Bäderbetriebe
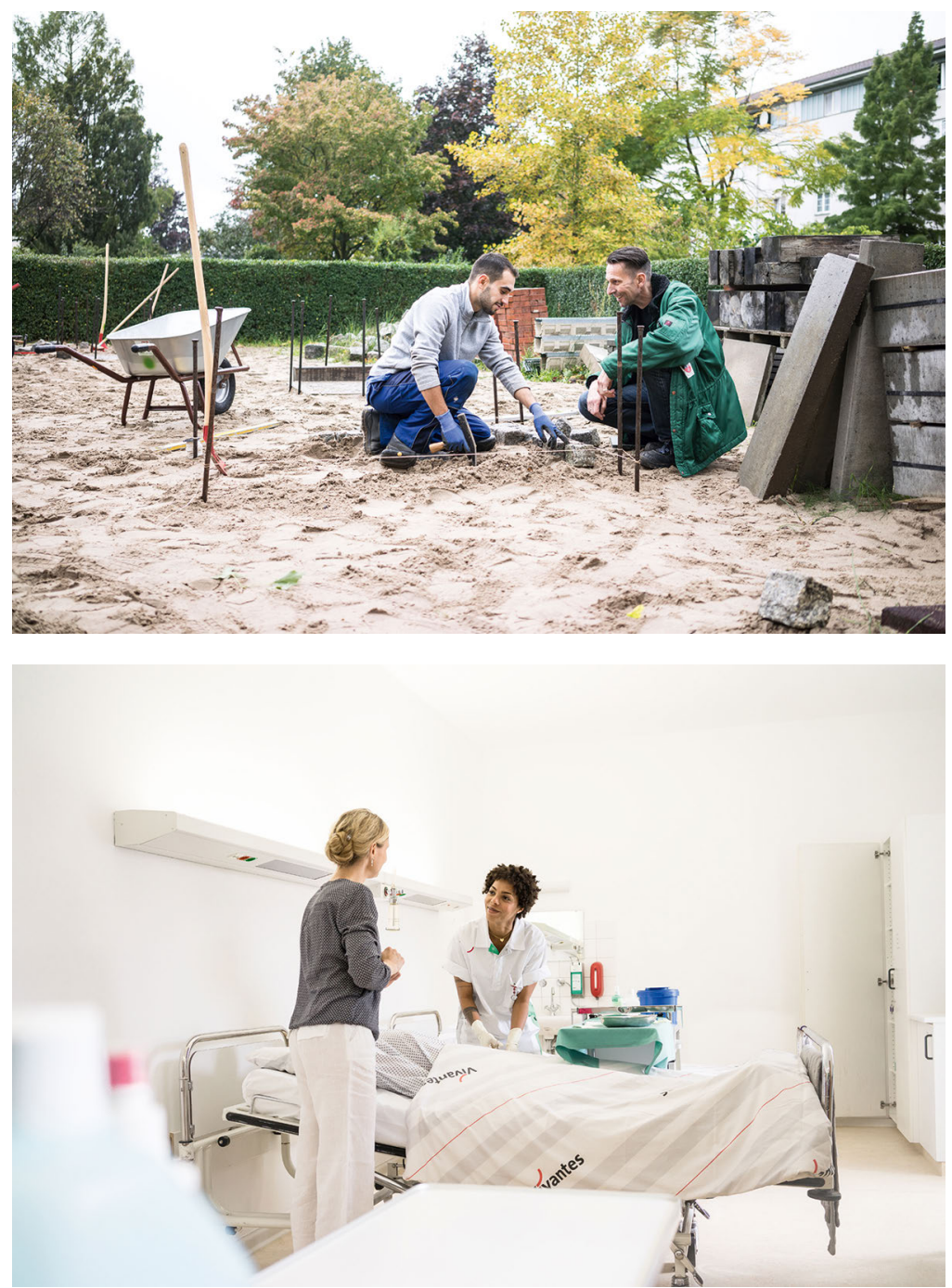

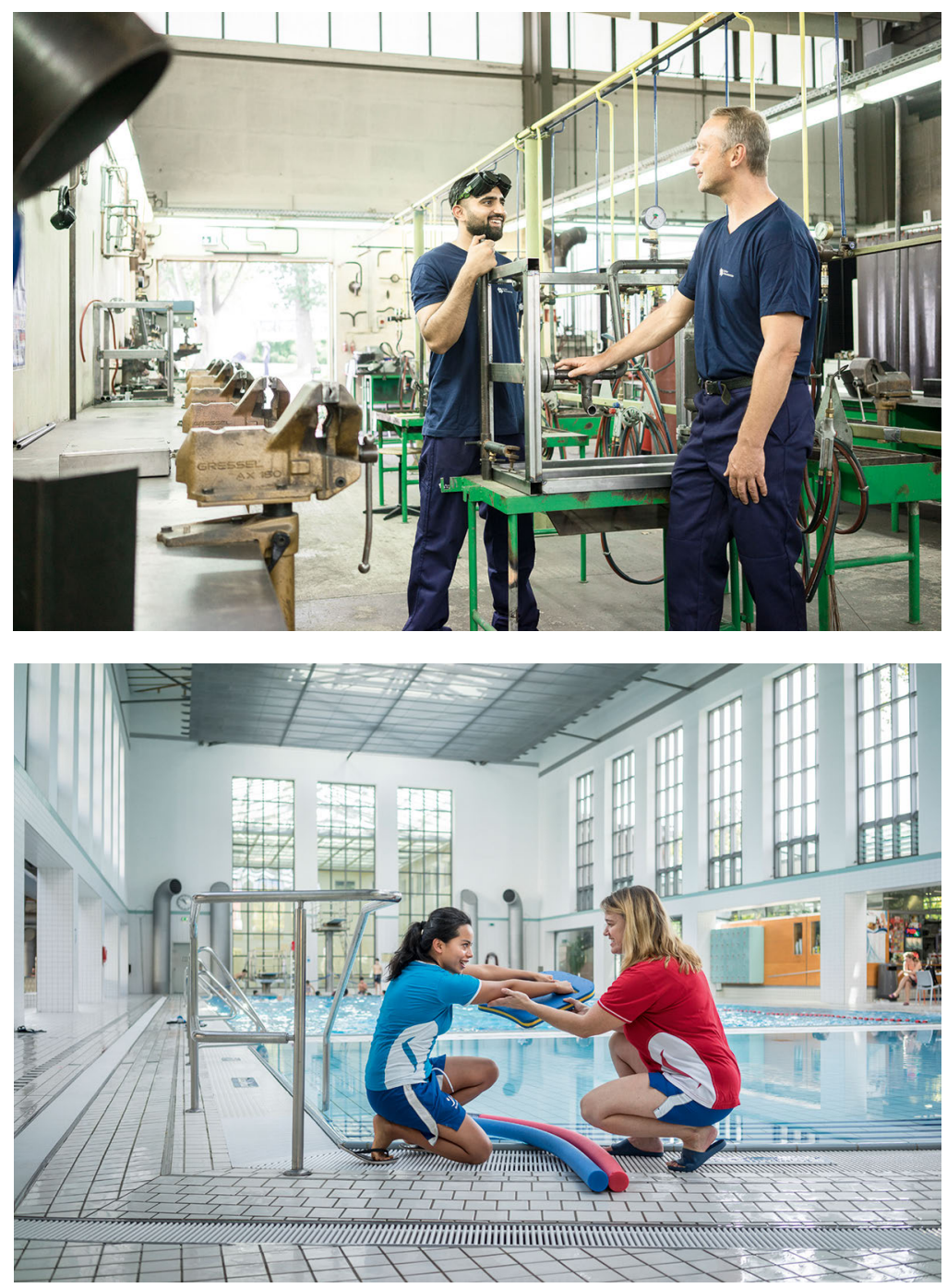

Copyright: BQN Berlin/Mikael Oskarsson 


\section{Fazit}

Im vorangegangenen Kapitel 3 wurde gezeigt, dass die Partnerschaften des Leitprojekts Berlin braucht dich! über Betriebe hinaus auf eine Gruppe von Sekundarschulen erweitert wurden. Damit entstand, was dann Berlin braucht dich! Konsortium genannt wurde. Diese auf eine längere Dauer gestellte enge Zusammenarbeit zwischen einer Gruppe von Betrieben und einer Gruppe von Schulen gehört zu den wichtigen Besonderheiten dieses integrationspolitischen Leitvorhabens. Worin diese Besonderheiten bestehen, wie sich die Zusammenarbeit entwickelt hat, welche Schwierigkeiten, aber auch Chancen sich aus der speziellen Zusammensetzung dieses Konsortiums ergeben haben und ob der Konsortial-Ansatz Potenzial über den konkreten Fall hinaus hat, ist Gegenstand dieses Kapitels gewesen.

Was hier Konsortium genannt wird, ist im »Lehrbuchsinn« nicht als eines entstanden, also nicht durch einen auf einer Vereinbarung basierenden Zusammenschluss von Partnern, sondern über das Vorhaben Berlin braucht dich! und damit über den Integrationsbeauftragten vermittelt worden. Insofern war »Konsortium« zu Beginn mehr eine Zuschreibung als ein bewusster Entschluss der Partner. Es hat sich aber verhältnismäßig rasch eine Art gemeinsames Selbstverständnis entwickelt, das vor allem aus den neuen Erfahrungen einer intensiven Zusammenarbeit und einem wachsenden wechselseitigen Verständnis zwischen Betrieben und Schulen, aber auch durch regelmäßige Treffen und die jährlichen »Konsortialtagungen« gespeist wurde.

In der Rückschau zeigt sich allerdings, dass gegenüber dem am Anfang rasch gewachsenen Verständnis einer gemeinsamen Mission, nämlich der Öffnung von Ausbildung für junge Menschen mit Migrationshintergrund, die selbstbewusste Mitsteuerung der Entwicklung von Berlin braucht dich! durch das Konsortium zurückblieb.

Die gemeinsamen Koordinierungs- und Steuerungsgremien entwickelten kaum Eigeninitiative bzw. erlahmten. Dies hatte u.a. zur Konsequenz, dass BQN als beauftragter Träger von Berlin braucht dich! in starkem Maße als unmittelbarer Organisator von Aktivitäten und umfassender Sicherer und Dienstleister - insbesondere bei der Vermittlung von Betriebsbegegnungs-/Praktikumsplätzen - und weniger als fachlicher Unterstützer in Anspruch genommen wurde. 
Die schwache Eigensteuerung des Konsortiums hängt vermutlich auch damit zusammen, dass es bislang keine verbindlichen Vereinbarungen zu seiner Arbeit gibt. Dennoch haben die intensive Zusammenarbeit und die positive Ansprache, die das Konsortium findet, eine offenbar nach wie vor belastbare Basis gemeinsamen Verständnisses und von kooperativem $\mathrm{Zu}$ trauen erzeugt.

Diese Haltung der Zugehörigkeit wirkt als Basis dafür fort, das Potenzial des Konsortiums als gemeinsame Innovationsplattform zu sehen und zu nutzen. Dies wird nun durch einen wichtigen empirischen Tatbestand herausgefordert, der nach einigen Jahren der Zusammenarbeit deutlich wurde: Zwar waren die Anteile von Jugendlichen aus Familien mit Einwanderungsgeschichte in den im Konsortium mitarbeitenden Betrieben gestiegen, aber den Schüler"innen aus den mitarbeitenden Integrierten Sekundarschulen gelang es immer noch nur sehr selten, einen Ausbildungsplatz in diesen Betrieben zu erhalten.

Berlin braucht dich! stand also vor dem schwierigen Sachverhalt, dass Gruppen von Betrieben und Schulen im Konsortium »zusammengespannt « waren, die zwar bei den Betriebsbegegnungen/Praktika im Rahmen der Berufsorientierung ganz gut harmonierten, aber bei der Besetzung der Ausbildungsplätze nicht zusammenkamen. Ob hierfür Lösungen gefunden werden können, wird zu einer weiteren Bewährungsprobe für das Konsortium und für das integrationspolitische Leitprojekt insgesamt. Welche Fortschritte bislang erreicht wurden, und was bislang nicht erreicht werden konnte, ist Thema des folgenden Kapitels 5, welche Lösungsansätze sich abzeichnen, wird in Kapitel 8 behandelt. 



\section{Kapitel 5}

\section{Auf dem Weg: Fortschritte und offene Fragen}

Die seit 2006 umgesetzte Kampagne hatte erhebliche initiale Wirkungen. Der durch Gestaltung der Plakate, durch Begleitveranstaltungen und ein entsprechendes Medienecho deutlich an Jugendliche mit Migrationsgeschichte adressierte Ruf »Berlin braucht dich! « und die damit verbundene erhöhte Aufmerksamkeit der beteiligten Betriebe gegenüber solchen Bewerber*innen führte zu einem raschen Anstieg ihres Anteils an den Auszubildenden. Er blieb aber noch weit unter dem senatsseitig formulierten Ziel, dass dieser Anteil so hoch sein solle wie der Anteil von Migrant*innen an der Berliner Bevölkerung, damals ca. 25 Prozent. Damit war senatsseitig eine klare Erwartung formuliert, auf eine Quotenvorgabe aber ausdrücklich verzichtet worden.

\section{Den Impuls der Kampagne sichern}

Nun ist es ein Charakteristikum von Kampagnen, dass sie sich erschöpfen. Eine weitere Vermutung war, dass die Kampagne vor allem bei jenen Jugendlichen gut angekommen und aufgegriffen worden war, die ohnehin schon »auf dem Weg« waren, gewissermaßen nur auf ein solches Signal gewartet hatten und sich aufgrund ihrer schulischen Leistungen und ihres Verhaltens gute Chancen ausrechnen konnten, auch genommen zu werden. Die Kampagne hätte dann vor allem einen »Creaming-Effekt« gehabt, bei dem eine Abschöpfung der qualifizierten Jugendlichen stattfindet, ohne tiefer auf die Gruppe der Jugendlichen mit Migrationsgeschichte insgesamt einzuwirken. 
Eine integrationspolitisch entscheidende Frage war also, wie der Impuls auf Dauer gestellt werden könnte. Die Antwort darauf war die Entwicklung von Betriebsbegegnungen. Betriebsbegegnungen, die als Unterbau der Kampagne zur Öffnung der Ausbildung für Jugendliche mit Migrationsgeschichte bezeichnet werden, rücken in den Jahren zwischen 2010 und 2014/2015 ins Zentrum von Berlin braucht dich!. In dieser Zeit verschiebt sich für mehrere Jahre der Schwerpunkt des Vorhabens von der unmittelbaren Öffnung von Ausbildung für Jugendliche mit Migrationsgeschichte auf das Feld einer spezifisch akzentuierten Berufsorientierung für die Schuljahre 7 bis 10.

Schon die Bezeichnung "Betriebsbegegnungen«, die sich vom gebräuchlichen »Praktikum« absetzte, signalisiert, dass hier vor allem an ein wechselseitiges Bekanntwerden gedacht war: Die Schüler*innen lernen die Betriebs- und Berufswelt kennen und die Betriebe junge Leute mit Migrationsgeschichte, denn auf beiden Seiten wurde zu Beginn der 2010er Jahre von einer weitgehenden Fremdheit ausgegangen. Als Unterbau zur Bereitschaft der beteiligten Betriebe, ihre Ausbildung für Jugendliche mit Migrationsgeschichte $\mathrm{zu}$ öffnen, wurde dies insofern verstanden, als erwartet wurde, dass die wechselseitige Überwindung von Fremdheit bei den Schüler*innen zu einem vermehrten Interesse an Ausbildung, bei den Betrieben zu einer erweiterten interkulturellen Öffnung der Ausbildung führen würde.

\section{Betriebsbegegnungen und ihre Prämissen}

Den Betriebsbegegnungen wurde eine wichtige mittelfristige integrationspolitische Hebelwirkung zugetraut, dies allerdings nur unter bestimmten, die Qualität der Betriebsbegegnungen betreffenden vier Voraussetzungen:

Erstens sollte der Kampagnen-Impuls im Sinne einer explizit interkulturellen Öffnung der Betriebsbegegnungen fortwirken. Deswegen wurden als Anbieter diejenigen Betriebe und Verwaltungen ins Auge gefasst, die bei der »Kampagne« mitgewirkt hatten.

Zweitens sollte der Kontakt zur Berufs- und Betriebssphäre früh beginnen und systematisch auf- und ausgebaut werden. Deswegen wurde - wie bereits beschrieben ${ }^{1}$ - eine Abfolge verschiedener Typen von Betriebsbegegnungen von Klasse 7 bis Klasse 10 konzipiert. Damit wurde zu einem frühen

1 Vgl. Kapitel 1. 
Zeitpunkt die Idee einer schülerbiografisch längerfristig angelegten Berufsorientierung aufgenommen, die sich später in vielen Landeskonzepten finden sollte, insbesondere in der Landeskonzeption Berufs- und Studienorientierung, und sie wurde gewissermaßen radikalisiert, weil es nicht um verschiedene Formen außerschulischer Praxis, sondern explizit um das Praxisfeld Betrieb ging.

Drittens rückte die pädagogische Qualität der Betriebsbegegnungen ins Zentrum der Aufmerksamkeit, weil es darum ging (und geht), Schüler*innen aus betriebs- und ausbildungsfernen Milieus zu interessieren und zu motivieren, und zwar möglichst so stark, dass dies insgesamt einen Schub für stabile Lernbereitschaft gibt.

Viertens mündeten - vor dem Hintergrund der integrationspolitischen Ziele - diese drei Prämissen zur Abkehr von einem bei vielen Expert*innen zum Übergang von der Schule in die Arbeitswelt bis dato unerschütterlichen Glaubenssatz, nämlich, dass die Schüler*innen sich ihre Praktikumsplätze selbst suchen sollen. Denn im Ergebnis führte die eigenständige Suche von Schüler*innen nach einem Praktikum oftmals zu solchen Plätzen, die von ihrer Qualität nicht den Motivationsschub brachten, der gerade für die in diesem Kontext in den Blick genommenen Schüler*innen so wichtig ist. Manchmal war der Effekt sogar gegenteilig, weil schlechte Praktika eher abschrecken als motivieren.

Wie konnten diese Überlegungen ins Werk gesetzt werden? Zunächst mussten die Kampagnen-Betriebe dafür gewonnen werden, diese Art von Betriebsbegegnungen anzubieten. In mehreren Schritten entwickelte BQN mit Ausbildungsexpert"innen aus den Betrieben gemeinsam die unterschiedlichen Typen von Begegnungen, die nacheinander folgen sollten unter Einschluss des bereits obligatorischen dreiwöchigen Praktikums in Klassenstufe 9 und basalen Qualitätskriterien. Betrieben, die bereits vielfältige und langjährige Erfahrungen mit Schülerpraktika hatten, fiel es leichter als anderen, sich Angebote vorzustellen und diese zu realisieren. Eine ganze Reihe von Kampagnen-Betrieben zeigten sich bereit, mitzuwirken und im Rahmen ihrer Angebotspalette von Praktika ein solches neues »Segment« zu platzieren und zu reservieren. 


\section{Kooperation mit Schulen: Welche Schulen?}

Wie war sicherzustellen, dass diese Betriebsbegegnungsplätze nun an jene kamen, um die es ging und geht: um junge Leute mit Migrationsgeschichte, die von allein und aus eigenem Antrieb noch nicht »auf dem Weg« sind, sondern hierfür noch einen nachhaltig wirksamen Schub benötigen. Zugleich war klar, dass die gewünschte Abfolge von Betriebsbegegnungen über die verschiedenen Klassenstufen ohne eine Einfügung in die jeweiligen schulischen Abläufe von Berufsorientierung nicht zu machen sein würde. Es mussten also Sekundarschulen als Partnerinnen gewonnen werden. Damit wurde die Kampagne zu einem Kooperationsprojekt.

Doch welche Schulen sollten angesprochen und nach ihrem Interesse an Betriebsbegegnungen dieses neuen Typs für ihre Schülerinnen und Schüler befragt werden? Die Teilnahme wurde unter den Sekundarschulen ausgeschrieben und diese konnten sich bewerben. Aus dem Büro des Integrationsbeauftragten wurde dafür plädiert, Sekundarschulen zur Teilnahme aufzufordern, die einen besonders hohen Anteil von Schüler*innen mit Migrationsgeschichte aufwiesen. Im Ergebnis waren es 26 Sekundarschulen aus den Innenstadtbezirken der Stadt. Dass diese Schulen neben hohen Quoten von Schüler*innen aus Einwanderungsfamilien auch hohe Quoten von Lehrmittelbefreiung aufwiesen, was ein Indikator für SGB-II-Bezug ist, war ursprünglich nicht intendiert. Es spiegelt aber eine Berliner Realität wider, in deren Innenstädtischen Bezirken soziale und ethnische Abgrenzungen einander überlappen.

\section{Erste Kooperationserfahrungen und die Erfindung des "matching-tool«}

Nun mussten Wege der Zusammenarbeit zwischen den beteiligten Betrieben und Schulen gefunden und erprobt werden. Die erste gemeinsame Konsortial-Tagung brachte die Erkenntnis, dass Schulen und Betriebe zwei sehr unterschiedliche Welten sind, die wenig voneinander wissen, aber einander vieles zuschreiben. Die wechselseitigen Berichte und Einblicke brachten viele »Aha«- Effekte und auf diese Weise eine wachsende Bereitschaft, jungen Leuten mit Migrationsgeschichte aus diesen Schulen in diesen Betrieben Be- 
triebsbegegnungen zu ermöglichen. Rasch ging man daher zu praktischen Fragen über.

Die grundsätzlich zu klärende Frage war, wie Schüler*innen an welche Betriebsbegegnungen kommen. Daneben kam im Austausch eine Fülle weiterer pragmatischer Aspekte auf, die beantwortet werden mussten: Kommen die Zeiten, in denen Betriebe solche Begegnungen anbieten können, und die Zeiten, die in den Schulen für außerschulische Aktivitäten möglich sind, zur Deckung? Gibt es Ausschlüsse aus bestimmten Begegnungen aufgrund des Alters der Schüler*innen? Finden die Schüler*innen überhaupt allein und pünktlich die Betriebe? Wie sieht die Betreuung durch die Lehrer/innen aus? Müssen Vereinbarungen abgeschlossen werden? Eine Koordinierungsgruppe aus Betriebs- und Schulvertreter*innen befasste sich gemeinsam mit BQN intensiv mit diesen Fragen. Es entstanden Merkblätter und Leitfäden.

Mit der wachsenden Zahl der angebotenen Betriebsbegegnungen auf den verschiedenen Klassenstufen kamen die Einzelverabredungen über die Vermittlung von Schüler*innen in die jeweiligen Betriebsbegegnungen rasch an ihre Grenze. Außerdem sollte eine Zweierpartnerschaft zwischen einem Betrieb und einer Schule vermieden werden, weil dies zu einer fachlich-beruflichen Einengung des Angebots für die beteiligte Schule und zu einer möglichen zu frühzeitigen Bindung der Schüler*innen an einen Betrieb geführt hätte. Deshalb sollte in einer sehr ambitionierten Perspektive zumindest potenziell das gesamte berufliche Angebotsspektrum aller Betriebe allen Schüler*innen zur Verfügung stehen.

Im Ergebnis wurde nach einer Art »technischen Lösung« der komplexen Herausforderung der Vermittlung gesucht: entwickelt wurde ein »matching-tool«, das mit allmählicher Perfektionierung zuließ, dass Schulen elektronisch Angebote von Betrieben zu Betriebsbegegnungen in einer vorher bestimmten Größenordnung »buchen« konnten. Die ständige Weiterentwicklung und Pflege des »tools« und die dennoch nötige aufmerksame persönliche Begleitung und Intervention wurde von $\mathrm{BQN}$ übernommen eine aufwendige Aktivität, die erhebliche Ressourcen band.

Wie so oft, ist auch hier das Konzept die eine Seite und die Umsetzung die andere. Wobei "Umsetzung" in diesem Zusammenhang im Grunde ein zu technisches Verständnis nahelegt, als ginge es »nur« darum, die richtigen Schritte zu tun und die richtigen Instrumente zur Verfügung zu haben und einzusetzen. Tatsächlich aber handelt es sich um ein »soziales Experiment«, in das die Motive und Handlungsweisen der beteiligten Akteure einfließen 
und auf das auch die gesellschaftlichen Umstände als wichtige Rahmenbedingungen einwirken. Einige dieser Faktoren, die die erste Periode der durch Berlin braucht dich! gestifteten Zusammenarbeit zwischen Schulen und Betrieben beeinflusst haben, sollen hier kurz diskutiert werden.

\section{Betriebe und Schulen »ticken « unterschiedlich}

Zunächst und ganz generell sind Betriebe und Schule Systeme oder Subsysteme, die ganz unterschiedlichen Logiken folgen, die in der Zusammenarbeit oft genug auch konflikthaft aufeinanderstoßen. Hierzu gehört auf der Seite der Betriebe, dass diese darüber entscheiden, wen sie einstellen und entsprechend auch, wen sie ausbilden. Dieser Personalauswahl-Vorbehalt spielt eine wichtige Rolle und gilt im Prinzip schon für die Betriebsbegegnungen, denn sie finden in den Lokalitäten des Betriebs statt, binden personelle Kapazitäten und berühren die betrieblichen Abläufe. Üblicherweise steht das Angebot an mehrwöchigen Praktikumsplätzen in Klassenstufe 9 schon unter der Überlegung, Auszubildenden-Nachwuchs zu gewinnen. Deswegen müssen Schüler*innen sich oft - z.T. aufwendig - in den beteiligten Betrieben für ein Praktikum bewerben. Die Bereitschaft von Betrieben, für die Betriebsbegegnungen im Kontext von Berlin braucht dich! weitgehend auf eine solche Auswahl zu verzichten, gibt diesen - zahlenmäßig beschränkten - Praktikumstyp, der im Gesamt der betrieblich angebotenen Praktika meist nur einen kleinen Teil ausmacht - innerbetrieblich oftmals eine Art "Sonderstatus« als eine besondere sozialpolitisch oder integrationspolitisch motivierte Maßnahme.

\section{Bilder und Botschaften}

Im Rückblick wird deutlicher, dass in Hinblick auf die Praktikant"innen, die über Berlin braucht dich! kamen, für viele der beteiligten Betriebe das sozialpolitische Motiv stärker im Vordergrund stand als die Erwartung, aus diesen Kreisen tatsächlich eigenen Ausbildungsnachwuchs gewinnen zu können. Dies muss vor dem Hintergrund gesehen werden, dass mit der Entscheidung für bestimmte Sekundarschulen als Partnerinnen nun konkrete Schulen und konkrete Schülerschaften ins Spiel kommen. Schulen und Schülerschaften 
wird oftmals ein bestimmtes Image zugeschrieben, das z.T. auch dort selbst »verinnerlicht« ist und jedenfalls bei jeder Begegnung »mitwandert«.

Auf der anderen Seite lösen die kooperierenden Betriebe und Verwaltungen ebenfalls Bilder aus. In unserem Kontext gehören alle Betriebe und Verwaltungen, die in Berlin braucht dich! kooperieren, zu jenen Arbeitgebern, die bislang sowohl für die Schüler*innen als auch für die Lehrer*innen unerreichbar schienen. Es konnte oft beobachtet werden, dass Lehrer*innen für diese Plätze nur solche besonders guten und vormotivierten Schüler"innen vorschlagen, von denen sie annehmen, dass sie dort bestehen können: wenigstens für diese sollte sich ihr Spektrum an Optionen erhöhen! Jene, die möglicherweise noch dringender ein gutes Praktikum benötigt hätten, blieben auf diese Weise zurück.

Die in dieser Phase mit Berlin braucht dich! verbundene doppelte Botschaft einer starken Motivation, die allein schon durch die Begegnung mit betrieblicher und beruflicher Wirklichkeit hervorgerufen werde, und der wirklichen Stärken der Jugendlichen, die sich erst in der nicht-schulischen praktischen Ernstsituation zeigen würden, bewegte viel. Aber sie war zu wenig auf die betrieblichen und schulischen Rahmenbedingungen in vielen dieser sozial stark belasteten Schulen und die sozio-biografischen Kontexte der Jugendlichen bezogen und damit - wenn man so will - zu idealistisch. Die Folge war eine Überschätzung der Praxiswirkung der Betriebsbegegnungen, die doch tatsächlich im Leben der Schüler*innen eher punktuelle Ereignisse blieben.

\section{Schwierig: Ein realistischer Blick auf die Jugendlichen}

Auf die Kraft der Betriebe und die verborgenen Stärken der Jugendlichen allein zu setzen, hatte in dieser Zeit noch einen anderen Grund, nämlich den, einer Haltung der Verteidigung der Jugendlichen mit Migrationsgeschichte und aus bestimmten Milieus einzunehmen. Denn medial wurden laute und skandalisierende Debatten um kriminelle Jugendbanden, schwer zu leitende Schulen, verzweifelte Lehrer und gewalttätige und unsoziale junge Leute geführt. ${ }^{2}$ Aus dieser Verteidigungshaltung heraus, in der es zugleich auch

2 Beispiele für Veröffentlichungen, die Material und Bezug für diese öffentliche Aufmerksamkeit lieferten, sind u.a. schon früh Pick, Brigitte: Kopfschüsse: Wer PISA nicht versteht, 
um sprachliche Vermeidung von Diskriminierung ging, gelang kaum die Entwicklung eines nicht-diskriminierenden, aber realistischen Blicks auf die Stärken und die Schwächen und vor allem auch auf die Verletzlichkeit von Motivation und Durchhaltevermögen bei diesen Jugendlichen - eine Verletzlichkeit, die auch Produkt der weitergegebenen Erfahrungen aus den sozialen Milieus, in denen sie aufwachsen, sind. Als Konsequenz der Verteidigungshaltung spielte jedenfalls die sozial-räumliche Einbettung von Berlin braucht dich!, die grundsätzlich als wichtig erachtet wurde, in diesen Jahren kaum eine Rolle.

\section{Beim Start: Fehlende oder schwache Einbettung in schulische Berufsorientierung}

$\mathrm{Zu}$ Beginn dieser Phase wurden die Betriebsbegegnungen - ideal in ihrer Viererfolge - als aufeinander aufbauendes »System « betrachtet, das aus sich heraus orientierende und Entscheidungen fördernde Wirkungen erzielen kann, vorausgesetzt, dass dieselben Schüler*innen jeweils auch die vier Stufen nutzen können.

Schon dies war eine vor allem aus zwei Gründen schwierige Prämisse. Zum einen war das Angebot an Betriebsbegegnungen in den vier Jahrgängen quantitativ unterschiedlich stark und besonders schwach für einwöchentliche Schnupper-Praktikum ausgeprägt, das für die Jahrgangsstufe 8 konzipiert war. Zum anderen - und noch wichtiger - stellte aber die Idee einer kontinuierlichen Nutzung der Betriebsbegegnungen über die aufeinanderfolgenden Jahrgangsstufen hinweg eine große Herausforderung dar. Denn dies setzt im Grunde voraus, dass die Schüler*innen bei der Entwicklung ihrer Berufsorientierung durch die Schule individuell und kontinuierlich begleitet, aber nicht gedrängt werden.

Individuelle Berufsorientierungs-Entwicklungspläne mit dem Ziel des Aufbaus von Entscheidungsfähigkeit wären von Anfang an erforderlich gewesen. Diese fehlten aber als ein systematischer schulischer Ansatz, wenn-

muss mit RÜTLI rechnen, Hamburg 2007; Rogg, Ursula 2008: Nord-Neukölln. Frontbericht aus dem Klassenzimmer, München 2008 Heisig, Kirsten : Das Ende der Geduld. Konsequent gegen jugendliche Cewalttäter, Freiburg, Basel, Wien 2010 sowie die bereits erwähnten Bücher T. Sarrazin: Deutschland schafft sich ab und H. Buschkowsky: Neukölln ist überall. 
gleich sich einzelne Lehrer*innen im Rahmen ihrer sehr eingeschränkten zeitlichen und Kraft-Ressourcen z.T. intensiv um die individuellen Entwicklungen von Schüler*innen bemühten. Genereller gesagt, wäre es von Anfang an um eine gute Einbettung der Betriebsbegegnungs-Abfolge von Berlin braucht dich! in ein ausgebautes System der schulischen Berufsorientierung gegangen. Die anfängliche Überschätzung der motivierenden Wirkungen von - punktuellen, aber in einer vierjährigen Kontinuität stehenden - Betriebsbegegnungen verdeckte zunächst zusätzlich die damaligen systematischen schulischen Mängel bei der Berufsorientierung.

In diesen Jahren entwickelte sich Berufsorientierung auch an den beteiligten Schulen durchaus weiter, wies (und weist) aber nach wie vor insbesondere unter systemischen Aspekten deutliche Defizite auf. Im Ergebnis blieben die Betriebsbegegnungen, so gut sie auch sein mochten, eine Art isolierter Erfahrung im ansonsten anders bestimmten Schulalltag sowie in der Lebenswirklichkeit und der persönlichen Entwicklung der Schülerinnen und Schüler.

Dies ist umso problematischer, als sich die Schulzeit zwischen dem 7. und 10. Jahrgang und insbesondere die frühen Jahre mit dramatischen Entwicklungen der jungen Persönlichkeiten zusammenfallen: Pubertät, der langsamen Ablösung von den Eltern und der allmählichen Herausbildung einer Perspektive auf das eigene Erwachsenenleben. Auch diese psycho-sozialen Entwicklungsprozesse, die Turbulenzen und Instabilitäten mit sich bringen, wurden anfangs beim Konzept der Vierstufigkeit wenig berücksichtigt.

\section{Qualifizierte Vierstufigkeit: gut gedacht, schwer umzusetzen}

Bei Qualifizierter Vierstufigkeit, wie sie sich als Formel später herausbildete, musste es also nicht nur um die Qualität der Betriebsbegegnungen im engeren Sinne, sondern um ihre Einbettung in ein gutes schulisches System der Berufsorientierung gehen, im dem seinerseits pädagogisch die Entwicklungsdynamik jugendlichen Lebens in dieser Phase reflektiert wird. Die mehrfache Isoliertheit des Betriebsbegegnungsansatzes begrenzte in den Anfangsjahren seine durch die Zentralität von direkter Betriebs- und Berufserfahrung mögliche positive Wirksamkeit.

Im Rückblick wird allerdings auch eine gewisse Isoliertheit des Betriebsbegegnungs-Ansatzes oder Sonderrolle in den beteiligten Betrieben selbst 
erkennbar. Zwar beteiligten sich betriebliche Expert"innen vor allem in der Einsteuerungsphase intensiv an der Entwicklung des Modells und seiner Qualität. Dies geschah aber weitgehend ohne Konsequenzen für das restliche Praktika- und Ausbildungsgeschehen und diente kaum als Anregung für den eigenen systematischen Ausbau von Praktika als Voraussetzung für das künftige Gewinnen von Auszubildenden - trotz der sich abzeichnenden Verknappung der traditionellen Bewerberschaft um Ausbildung.

Ob und wie die Qualifizierte Vierstufigkeit funktioniert, hängt also in erheblicher Weise davon ab, wie Schulen und Betriebe damit umgehen, von ihrer aktiven Rolle in der parallelen weiteren Ausgestaltung korrespondierender Teilsysteme von Berufsorientierung. Dies kann man auch als erhebliche Erwartungen an Schulen und Betriebe lesen. Deshalb sei an dieser Stelle betont, dass weder Schulen noch Betriebe für ihre Beteiligung an Berlin braucht dich! eine finanzielle Förderung erhielten.

\section{Berlin braucht dich!-Plätze: immer kontingentiert}

Eine weitere limitierende Bedingung des Vorhabens wurde intern schnell erkannt, ohne ihr beikommen zu können: Die Kontingentierung der Angebote zur Betriebserkundung. Was ist damit gemeint? Die beteiligten Betriebe speisen jeweils nur eine gewisse begrenzte Anzahl von Betriebsbegegnungen der verschiedenen Stufen ein; daraus ergibt sich das Gesamtangebot, das den beteiligten Schulen zur Verfügung steht.

Damit die Verteilung auf die Schulen einigermaßen fair ist und zugleich die verschiedenen Jahrgangstufen und mehrere Berufsfelder abdeckt, werden in einem komplizierten Kommunikationsverfahren, das "Matching" genannt wird, für jede Schule Kontingente definiert, die schulintern zur Verteilung kommen. Als Resultat ergibt sich auf der Seite der einzelnen Schulen, dass die über Berlin braucht dich! kommenden Betriebsbegegnungen nur einer kleinen Anzahl von Schüler"innen zugutekommen.

Geht man davon aus, dass alle Schüler*innen Betriebsbegegnungen brauchen würden, dann bringt Berlin braucht dich! nur einen kleinen Anteil an dieser - potenziellen - Gesamtnachfrage. Besonders augenfällig wird dies beim dreiwöchigen obligatorischen Praktikum der Klassenstufe 9: Alle müssen ein Praktikum machen; diejenigen, die es vermittelt über Berlin braucht dich! erhalten, machen davon nur eine kleine Gruppet aus. Zugleich 
ist davon auszugehen, dass die Betriebsbegegnungen aus dem Berlin braucht dich!-Kontext eine überdurchschnittlich gute Qualität aufweisen und die anbietenden Betriebe auch als potenzielle Ausbildungsbetriebe besonders attraktiv sind.

Die Frage ist also, wie die Schulen diese Plätze nutzen. Es spricht, wie oben bereits angedeutet, vieles dafür, dass vor allem Schüler"innen zum Zuge kamen, von denen die Lehrer*innen und sie selbst am ehesten erwarteten, dass sie in diesen Betrieben bestehen und dies für sich nutzen können, oder anders: die den Erwartungen, die Betriebe an Schülerpraktikant"innen haben, am ehesten entsprachen. Soweit erkennbar, fand also oftmals in diesem Sinne eine vor allem an den bei den Betrieben vermuteten Erwartungen orientierte »Bestenauslese« statt.

\section{Gute Betriebsbegegnungen für alle?}

Damit stellte sich und stellt sich die Frage, wie in den Schulen die zur Verfügung stehenden Plätze von Praktika/Betriebsbegegnungen besser "verteilt« werden. Denn im Grunde müsste eine "Gesamtabdeckung", also ein Angebot von Betriebsbegegnungen guter Qualität, für alle geben, das von Berlin braucht dich! Aber auch dann, wenn dieses erheblich expandiert, niemals allein beigebracht werden könnte.

Es ergeben sich also eine Reihe von - offenen - Fragen: Wie können die Schulen zu einer »Gesamtabdeckung « kommen, also zu Betriebsbegegnungen ausreichender Qualität auf jeder Klassenstufe für alle? Welchen Schüler*innen sollen dann die über Berlin braucht dich! kommenden Betriebsbegegnungen zugutekommen? Können die über Berlin braucht dich! kommenden Betriebsbegegnungen im »Pool « aller bei einer Schule erreichbaren Betriebsbegegnungen die Rolle eines »Qualitätstreibers« spielen und wenn ja, wie? Können - umgekehrt - die Erfahrungen, die die Schulen auch vergleichend mit den über Berlin braucht dich! gekommenen Betriebsbegegnungen machen, als Impuls in die betriebliche Gestaltung der Begegnungen/ Praktika zurückgespiegelt werden? Diese Fragen sind nicht neu; aber ihre Beantwortung steht nach wie vor aus und kann nicht exklusiv, sondern nur auf der Basis von breiten kooperativen Ansätzen erfolgen 


\section{"matching, matching ..."}

Mit der Etablierung des matching-tools als ein digital gestütztes komplexes Kommunikations- und Vermittlungsverfahren zwischen Schulen und Betrieben verschiebt sich die »Architektur« der Zusammenarbeit zwischen Betrieben, Schulen und dem beauftragten Träger BQN: Dieser wird de facto jeweils für mehrere Wochen zur »Matching-Agentur«, was in dieser Zeit andere wichtige Aufgaben zurückdrängt. Da das reibungslose Funktionieren auf zahlenmäßig hoher Stufenleiter zu einem wichtigen Erfolgskriterium für Berlin braucht dich! avanciert, wird dessen Absicherung zu einer Art von Leistungsnachweis für den Träger; wenn z.B. Schüler*innen am Tag des Beginns nicht pünktlich oder überhaupt nicht im Betrieb erscheinen, wird dies ebenso zur Sache des Trägers BQN gemacht wie Verhaltensauffälligkeiten im Betrieb selbst usw.

Auf diese Weise wird BQN immer mehr zum Dienstleister für Betriebe und Schulen. Das hat verschiedene Folgen: Die gemeinsame Arbeit an der Qualität der Begegnungen und an der besseren Einbettung in das schulische Geschehen - zeitweilig sehr lebendig - erlahmt, Verfahrensdiskussionen drängen den fachlichen Austausch zurück. Damit wird die Zusammenarbeit zwischen Betrieben und Schulen, also der konsortiale Impuls, zunehmend weniger genutzt.

\section{Schieflage und Ernüchterung}

Das Engagement auf beiden Seiten - Schulen wie Betriebe - wirkte zeitweilig ziemlich gebremst. Wie ist das zu erklären? Durch die Routine des "matching « war ein Weg gefunden worden, die für beide Seiten - Betriebe und Schulen - mit Berlin braucht dich! verbundenen begrenzten Optionen "zu verwalten«. Dies war auch Ausdruck der schon beschriebenen, in diesen Jahren aktuellen Schieflage zwischen den beteiligten Betrieben und den beteiligten Schulen. ${ }^{3}$

Die Teilnahme der Betriebe und Verwaltungen an Berlin braucht dich! war primär aus einer öffentlichen Verantwortung heraus und sozial- bzw. integrationspolitisch motiviert und konkretisierte sich in der - limitierten

3 Vgl. hierzu insbesondere Kapitel 4. 
- Bereitstellung von Betriebsbegegnungen. Obwohl sich auch bei diesen Betrieben und Verwaltungen in absehbarer Zukunft Personalengpässe und Schwierigkeiten bei der Besetzung von Ausbildungsplätzen abzeichnen, war deren aktuelle Motivation nicht so stark, um eine größere Anzahl junger Menschen aus diesen Schulen als künftige Auszubildende und Nachwuchs für qualifizierte Fachtätigkeit ins Auge zu fassen Das Bild, das viele der Lehrer*innen und Schulleiter*innen von ihrer Schülerschaft hatten, entsprach dem. Sie sahen für ihre Schüler*innen in diesen Betrieben und Verwaltungen nur in Ausnahmefällen Chancen auf einen Ausbildungsplatz.

Nach mehreren Jahren, in denen die Betriebsbegegnungen - wenngleich, wie schon erläutert, selten in der Weise einer kontinuierlichen Abfolge, wie sie die »Vierstufigkeit« konzeptionell vorsieht - "gematcht « und praktiziert wurden, ergab eine Zwischenbilanzierung 2014/2015 tatsächlich: Nur wenige der Schüler*innen, die an Betriebsbegegnungen aktiv beteiligt waren und sich bei den mitwirkenden Betrieben beworben hatten, erhielten einen Ausbildungsplatz. Obwohl dies "irgendwie« erwartet werden konnte, trat doch eine erhebliche Ernüchterung ein. Denn es wurde nun faktisch sichtbar, dass das mit Berlin braucht dich! von Beginn an verbundene Ziel der Öffnung von Berufsausbildung für Jugendliche mit Migrationsgeschichte jedenfalls zwischen den am Konsortium beteiligten Betrieben und Schulen bis zu diesem Zeitpunkt nicht erreicht worden war. Wohl aber zeigten die steigenden Anteile von Auszubildenden aus Familien mit Einwanderungsgeschichte in der Ausbildung dieser Betriebe, dass von Berlin braucht dich! sehr viele positive indirekte Effekte ausgegangen waren.

\section{Weiter mit denselben Partnern?}

Eine genauere Analyse der Gründe, die später unter der Formulierung »Benachteiligung ist hartnäckiger als erwartet « zusammengefasst wurden, ${ }^{4}$ und mögliche Konsequenzen wurden allerdings durch die eingespielte Matching-Prozedur, die eine erhebliche konservative, also auf das bisher »Setting« fixierte Bindung zur Folge hatte, verzögert.

\footnotetext{
4 Vgl. hierzu u.a. das Kapitel 6 und die Broschüre: Der Beauftragte des Berliner Senats für Integration und Migration ( $\mathrm{Hg}$.): Alle mit dabei? Potenziale der Berufsausbildung für Jugendliche aus Einwandererfamilien, Berlin 2019.
} 
Zur Korrektur der Schieflage hätten sich möglicher Weise Veränderungen in der Struktur der Partnerschaft von Schulen und Betrieben angeboten. Die Betriebsseite hätte um Betriebe aus Branchen mit manifesteren Nachwuchssorgen erweitert werden können. Diese Funktion erfüllte die schließlich erfolgte Erweiterung um die Metall- und Elektroindustrie nicht; dafür fügte sie dem Vorhaben neben guten Praktikumsplätzen ein anderes wichtiges Element, nämlich die explizit mitgestaltende Rolle der Sozialpartner, bei. Es hätten auch andere Sekundarschulen gesucht und eingeladen werden können, deren Schülerschaft vordergründig bessere Voraussetzungen für einen erfolgreichen Eintritt in eine Berufsbildung in den beteiligten Betrieben mitgebracht hätten.

$\mathrm{Zu}$ solchen Entscheidungen kam es nicht. Zwar erfolgte später eine Ergänzung um einige weitere Betriebe aus anderen Branchen, diese blieb aber zahlenmäßig moderat. Tatsächlich wurde in den folgenden Jahren ab 2015/2016 versucht, die begonnene Arbeit mit denselben schulischen und betrieblichen Partnern auf eine neue Grundlage zu stellen. ${ }^{5}$ Und auch dies hat gute Gründe, vor allem drei: Erstens: Für die jungen Leute, die die Schulen besuchen, um die es hier geht, ist Berufsausbildung nicht die einzige, aber eine wichtige Option für ihr künftiges Leben. Damit niemand zurückbleibt, müssen ihnen gute Ausbildungschancen eröffnet werden. Insofern dürfen diese Schulen nicht noch randständiger werden. Deshalb ist es gut, sie in Berlin braucht dich! zu halten. Zweitens: Die beteiligten Betriebe und Verwaltungen sind durch die Bank sehr gute Ausbildungsbetriebe. Als öffentliche Betriebe haben sie eine herausgehobene Verantwortung. Wer käme mehr als sie infrage, eine so gute pädagogische und menschliche Qualität von Ausbildung zu bieten, dass diese Jugendlichen sich entwickeln, erfolgreich ihre Ausbildung abschließen und ggf. auch als ausgebildete Fachkräfte für den Betrieb gewonnen werden können. Drittens: Die Zusammenarbeit zwischen Betrieben und Schulen, systematisch unterstützt durch BQN, hat sich über Jahre bewährt, wechselseitiges Verständnis und Vertrauen sind aufgebaut. Das Konsortium hat das Potenzial, gemeinsam die schwierige Aufgabe zu bewältigen, für und mit den Jugendlichen, die diesen Weg gehen wollen, erfolgreich Übergänge und Ausbildung zu gestalten.

5 Vgl. hierzu das Kapitel 8. 


\section{Fazit}

Dieses fünfte Kapitel ist ausführlich und differenziert der Entwicklung des Leitprojekts Berlin braucht dich! von seinem Start bis in die Jahre 2014/2015 nachgegangen. Diese Periode ist dadurch gekennzeichnet, dass aus einer von Betrieben aus dem öffentlichen Sektor getragenen Kampagne, die junge Menschen mit Migrationshintergrund animieren sollte, sich für eine Ausbildung zu bewerben, ein Kooperationsprojekt zwischen Betrieben und Integrierten Sekundarschulen wird. Wie im vorangehenden Kapitel gezeigt wurde, entsteht eine spezielle Variante von Konsortium.

Auf der integrationspolitischen Ebene blieb das Ziel, Ausbildung für junge Menschen mit Migrationsgeschichte zu öffnen, erhalten; de facto konzentrierten sich die Aktivitäten bei Berlin braucht dich! in diesen Jahren auf die Etablierung einer Abfolge von Betriebsbegegnungen ab Klasse 7, die später die Bezeichnung Qualifizierte Vierstufigkeit erhielt. Die von den beteiligten Betrieben bereitgestellten Plätze wurden über ein Matching-Verfahren, das vom Träger BQN organisiert wird, auf die beteiligten Schulen verteilt, was in jeder einzelnen Schule auf ein verhältnismäßig schmales Kontingent von über die im Konsortium mitarbeitenden Betriebe bereitgestellten Plätzen hinauslief.

Offenbar konnten beide Seiten im Konsortium, also Betriebe und Schulen, bei der Konzentration auf die Abfolge von Betriebsbegegnungen/Praktika mitgehen, weil ihnen plausibel war, dass dies die Orientierung von Schüler*innen auf und deren Interesse an Berufsausbildung verbessern würde. Auch die Sozialpartner der Berliner Metall- und Elektroindustrie sahen dies so und beschlossen, mit einer Reihe wichtiger Ausbildungsbetriebe bei Berlin braucht dich! mitzuwirken.

Rasch zeigten sich aber trotz der erheblichen und über die Jahre ansteigenden Zahl von Betriebsbegegnungen eine Reihe von Unzulänglichkeiten, die im vorliegenden Kapitel diskutiert wurden.

Als besonders problematisch zeigte sich erstens die Frage, wie und für wen die über die Betriebe im Konsortium kommenden Plätze in den Schulen genutzt werden, und zweitens, ob und in welcher Weise die Betriebsbegegnungen/Praktika in eine kontinuierliche schulische Berufsorientierung eingebettet sind. Die Schulen und deren Umgang mit Berufsorientierung rückten also verstärkt in die Aufmerksamkeit, während z.B. das gegebene 
Angebot an Betriebsbegegnungen nach Anzahl und Qualität eher als Rahmenbedingung akzeptiert wurde.

Trotz dieser Schwierigkeiten und offenen Fragen bringen diese Jahre fruchtbare und weiterführende Beiträge. Es entsteht ein integrationspolitisch motiviertes System von Berufsorientierung im Sinne einer Qualifizierten Vierstufigkeit, von dem Schülerinnen und Schüler an den mitarbeitenden Integrierten Sekundarschulen profitieren; dass dies integrationspolitisch von erheblichem Interesse ist, wird weitgehend anerkannt. Die Qualifizierte Vierstufigkeit nimmt Eingang in das in diesen Jahren entstehende Berliner Landeskonzept für Berufs- und Studienorientierung. Unterstützt und flankiert wird dies durch Leitfäden, Handlungshilfen und Filme, also einen ganzen Werkzeugkoffer, der die Erfahrungen aus diesen Jahren auch transferierbar macht.

Es ist sicherlich die gemeinsame Arbeit an der Ausgestaltung der Qualifizierten Vierstufigkeit, die das Konsortium - wie im vorangehenden Kapitel dargestellt - weiter zusammenwachsen lässt.

Nach mehreren Jahren wird aber auch deutlich, dass die Konzentration auf die Entwicklung der Berufsorientierung nicht dazu geführt hatte, dass es Schülerinnen und Schülern aus den im Konsortium mitarbeitenden Schulen in größerem Umfange gelungen wäre, in den Betrieben aus dem Konsortium einen Ausbildungsplatz zu erhalten.

Diese Diskrepanz zwischen wesentlich verbesserter Berufsorientierung und ausbleibendem Eintritt in eine Ausbildung musste zu einer gewissen Ernüchterung und zu kritischen Fragen gegenüber der bisherigen Praxis führen. Die Fortschritte, die im Feld der Berufsorientierung gemacht wurden und die weit über das Vorhaben Berlin braucht dich! hinaus ausstrahlten, verbieten es, von verlorenen Jahren zu sprechen. Dennoch ist deutlich, dass das Ziel der tatsächlichen Öffnung der Berufsausbildung - und damit auch der Beitrag, der von den Betrieben zu erwarten wäre - für die durch die mitarbeitenden Schulen ins Spiel gebrachten Jugendlichen, wenn auch nicht generell, aus dem Auge verloren wurde.

Es sind eine Reihe von (selbst-)kritischen Fragen, die nun zu klären waren: Ist unter den gegebenen Bedingungen eine Korrektur in Richtung steigender Übergänge aus den beteiligten Schulen in die Ausbildung der beteiligten Betriebe denkbar? Und wie könnte dies konzeptionell aussehen und umgesetzt werden? Werden die bisherigen gemeinsamen Erfahrungen 
hierfür hilfreich sein können? Eine Re-Orientierung stand also auf der Tagesordnung. Sie ist unter verschiedenen Aspekten Thema der folgenden Kapitel. 

Teil 2

Ausbildung tatsächlich für Vielfalt öffnen 



\section{Kapitel 6}

\section{Hartnäckige Benachteiligungen und die Bedeutung der schulischen Berufsorientierung}

In diesem Kapitel werden einige der Befunde, Argumente und Vorschläge aus einer Expertise wiedergegeben und kommentiert, die 2016 für den Berliner Integrationsbeauftragten erstellt wurde. ${ }^{1}$ Diese Expertise rückübersetzte Berlin braucht dich! in einen Kontext, der durch erhebliche fortbestehende soziale Ungleichheiten in Hinblick auf gelingende Übergange in weiterführende Bildung und Berufsausbildung gekennzeichnet ist, von denen Jugendliche mit Migrationsgeschichte überproportional häufig negativ betroffen sind.

\section{Der Übergang in Ausbildung bleibt schwierig}

In Hinblick auf die integrationspolitisch wichtige Frage danach, wie Risiken im Übergang abgebaut und für alle Jugendlichen gelingende Übergänge in ein selbständiges und selbstbestimmtes Leben gebahnt werden können, kommt - so die zentrale These - der Berufsausbildung tatsächlich eine besonders wichtige Funktion zu. Die potenzial kompensatorische Funktion, die Berufsausbildung einnehmen kann, hängt mit dem dort vorherrschenden Lernen in der betrieblichen Praxis zusammen. Dies leistet aber Berufsausbil-

1 Kruse, Wilfried: Übergänge von der Schule in die Berufsausbildung und Arbeitswelt: Integrationspolitische Gestaltungsaufgaben für die Periode 2016 folgende und die Rolle von Berlin braucht dich!, unveröffentlichtes Manuskript, Dortmund 2016. 
dung nicht schon a priori, sondern nur dann, wenn sie so gestaltet wird, dass auch Jugendliche mit ungünstigeren Startvoraussetzungen in ihr wachsen können, und zwar im Regelsystem und im Rahmen heterogener Auszubildendengruppen. Dies wird im Kapitel 7 vertieft diskutiert.

Kapitel 6 nimmt dagegen den im vorangegangenen Kapitel beschriebenen Tatbestand zum Ausgangspunkt: In den innerstädtischen Integrierten Sekundarschulen (ISS), die einen sehr hohen Anteil von Lernmittelbefreiung in Kombination mit einem sehr hohen Anteil von Schüler"innen mit Migrationsgeschichte aufweisen, sind die realen Einstiege in die duale Ausbildung nach wie vor im Durchschnitt sehr niedrig und um ein Mehrfaches niedriger als im Durchschnitt an allen anderen Berliner ISSen - und dies, obwohl auch der Anteil derjenigen, die nach Beendigung der Sekundarschule eine weiterführende Schule besuchen, ebenfalls niedriger ist. Damit ist dort der Anteil derjenigen, deren Schullaufbahnen weder direkt in Ausbildung einmünden noch in den Besuch einer weiterführenden Schule, besonders hoch. Allerdings gibt es auch Ausnahmen von der Regel, nämlich Schulen, die höhere Übergänge in Ausbildung ausweisen. ${ }^{2}$

Das Gesamtbild aber ist integrationspolitisch schon deshalb herausfordernd, weil es eben an diesen Schulen schon seit Jahren erhebliche Anstrengungen (und Ressourcenaufwendungen) gibt, diese Situation zu verbessern - nicht nur durch Berlin braucht dich!. Die vorangegangene Schulstrukturreform sollte mit ihrem neuen Abschluss »Berufsbildungsreife ${ }^{3}{ }^{3}$ die den Hauptschulabschluss ersetzt, sollte im Übrigen erreicht werden, dass die Schülerinnen und Schüler für den Übergang in berufliche Bildungsgänge qualifiziert sind. In den bisherigen Diskursen dominierte der Blick auf die

2 Dort werden oftmals Modellansätze praktiziert. Dieser Ansatz bleibt ambivalent, wenn nicht die Bedingungen für einen fairen Transfer in das Regelsystem mitbedacht werden. Vgl. Holtappels, Heinz Günter: "Schulentwicklung unter herausfordernden Bedingungen«, Auftaktveranstaltung mit den Projektschulen am 20.03.2015 in Essen, Universität Duisburg-Essen http://schulen-staerken.de/wp-content/uploads/2014/og/Auftaktveranstaltung-Holtappels.pdf

3 Zur Berliner Schulstrukturreform und als Übersicht über das Berliner Schulsystem: https:// www.berlin.de/sen/bildung/schule/bildungswege/; Die Ergebnissen der wissenschaftlichen Begleitstudie von 2017, deren Arbeiten geleitet wurden von Professor Dr. Jürgen Baumert (Max-Planck-Institut für Bildungsforschung - MPIB), Professor Dr. Kai Maaz (Deutsches Institut für Internationale Pädagogische Forschung - DIPF) und Professor Dr. Olaf Köller (IPN - Leibniz-Institut für die Pädagogik der Naturwissenschaften und Mathematik) finden sich unter www.dipf.de/de/forschung/projekte/berlin-studie 
Defizite, die die in diesen Schulen anzutreffenden Schülerinnen und Schüler in Hinblick auf die sogenannte "Ausbildungsreife aufweisen sollen. ${ }^{4}$ Einseitige Sichtweisen - hier auf die schulisch nicht behobenen Defizite, dort auf die Ausgrenzung dieser Schüler*innen durch die Auswahlpraxis der Betriebe - führen offenkundig nicht weiter.

Der Übergang in die Ausbildung stellt in gewisser Weise eine besondere »Nagelprobe« für das Bildungssystem dar, weil das duale Ausbildungssystem eben nach seiner Hauptseite hin, nämlich den vertraglichen Ausbildungsverhältnissen, privatwirtschaftlich organisiert ist. Schulische Berufsorientierung und ihre "Versprechen« erfahren hierdurch eine Realitätsprüfung, die außerhalb der schulischen Sphäre liegt, aber ihre Botschaften zurück in die Schule sendet. Es ist deswegen wichtig zu sehen, dass eine durchgreifend verbesserte schulische Berufsorientierung, die weitgehend ohne »Verwertung « im Sinne eines erfolgreichen Eintritts in Ausbildung bleibt, Gefahr läuft, in den Augen der Schüler"innen wie der Lehrer"innen an Prestige und damit auch an Motivierungskraft zu verlieren. ${ }^{5}$

4 Zum umstrittenen Begriff der »Ausbildungsreife« vgl. u.a. Dobischat, Rolf/Kühnlein, Gertrud/Schurgatz, Robert: Ausbildungsreife. Ein umstrittener Begriff beim Übergang jugendlicher in die Berufsausbildung (=Arbeitspapiere der Hans-Böckler-Stiftung 189), Düsseldorf 2012.

5 Deswegen ist auch Mecheril zuzustimmen, wenn er in seinem Kommentar zum Bremer Bericht »Bildung und Migration« schreibt: »Der Versuch, gesellschaftliche Verhältnisse durch `Pädagogik« positiv zu beeinflussen, ist nicht allzu selten ein trügerisches Inaussichtstellen von Chancen. Der Versuch hat nur dann eine Chance mehr zu sein, wenn er eingebettet ist in eine Cesamtstrategie, die etwa eine Stadtentwicklungspolitik umfasst, die darauf zielt, alle Wohngebiete zu respektablen Kontexten werden zu lassen oder Konzepte der nachhaltigen Reduktion von (Jugend-)Arbeitslosigkeit und der Schaffung von Ausbildungsplätzen umfasst.«Mecheril, Paul: Institutionen an die Schülerschaft anpassen, nicht umgekehrt - eine Einladung zur Kritik ausländerpädagogischer Förderung. Kommentar zum ersten Bildungsberichtsband für das Land Bremen »Bildung-Migration-soziale Lage. Voneinander und miteinander lernen.«https://www.transparenz.bremen.de/dokument/ bremen117.c.100929.de 


\section{"Segregierte Schulen"}

Der Blick auf die einzelne Schule ist zwar von Bedeutung, um ihre konkrete und besondere Situation zu verstehen und um weiterführende Ansätze $\mathrm{zu}$ identifizieren, aber die einzelne Schule ist keine autonome Welt für sich, sondern in Strukturen und übergreifende Entwicklungen eingebunden. Insofern gibt es immer Schulen, die eine erhebliche Schnittmenge von Gemeinsamkeiten aufweisen. Das Konzept der »Segregierten Schule« ist ein solcher Versuch, Rahmenbedingungen und Dynamiken bestimmter Schulen besser zu verstehen.

Als ssegregiert unter dem Aspekt von Herkunftsgeschichte der Schülerinnen und Schüler und sozialer Benachteiligung werden häufig Schulen bezeichnet, an denen der Anteil von Schülern mit Migrationshintergrund und sozial benachteiligten Schülern entweder überdurchschnittlich hoch oder unterdurchschnittlich niedrig ist. ${ }^{6}$ Segregation ist aber nicht gleichbedeutend mit Konzentration, sondern bezieht sich auf die Abweichung der Zusammensetzung der Schülerschaft von der sozialen Zusammensetzung des sie umgebenden Stadtteils oder der Stadt. Dies ist insofern wichtig, als damit gesamtstädtische sozial-räumliche Ungleichheiten und Prozesse von Chancenverteilung und Allokationen aufgerufen sind.

Im Bericht 2013 des Sachverständigenrat deutscher Stiftungen für Integration und Migration werden ausschließlich jene Schulen als >segregiert bezeichnet, ${ }^{7}$ die mehrheitlich (über 50 \%) Schüler*innen mit Migrationshintergrund unterrichten. Da diese im Schnitt häufiger aus Elternhäusern mit niedrigem sozioökonomischem Status kommen, sei der Anteil sozial benachteiligter Schüler*innen an segregierten Schulen aber oftmals sehr hoch.

Die Berliner Integrierten Sekundarschulen, um die es hier geht, gehören aufgrund der beiden generellen sozio-strukturellen Merkmale, die sie ha-

6 Der Begriff ,Segregation< im engeren Sinne bezieht sich auf die Entmischung von Menschen innerhalb eines Beobachtungsgebiets. Streng genommen sind also weder Schulen noch Stadtteile selbst als ssegregiert zu bezeichnen, sondern die Menschen, die darin leben. Vgl. Häußermann, Hartmut: „Wohnen und Quartier: Ursachen sozialräumlicher Segregation«, in: Ernst-Ulrich Huster, Jürgen Boeckh, Hildegard Mogge-Grothjan (Hg.): Handbuch Armut und soziale Ausgrenzung, Wiesbaden 2008, S. 335-349.

7 Sachverständigenrat deutscher Stiftungen für Integration und Migration: Segregation an deutschen Schulen: Ausmaß, Folgen und Handlungsempfehlungen für bessere Bildungschancen, Berlin 2013. 
ben, zu dem Schultyp, der im Bericht des Sachverständigenrats »Segregierte Schulen« genannt wird: Sie weisen alle sehr hohe "Segregationswerte« auf. Der Grad der Segregation differiert von Standort zu Standort. ${ }^{8}$ Integrationspolitisch muss überdies Beachtung finden, dass es in den letzten Jahren in einer Reihe dieser Schulen eine weitere Erhöhung bei beiden Merkmalen gegeben hat. Ganz generell werden für das Entstehen und die Befestigung segregierter Sekundarschulen drei Faktoren diskutiert: die wohnräumliche Situation, die Schulwahl und der Übergang in die Sekundarschule.

\section{Stadtteile und Quartiere}

Stadtsoziologische Forschungen weisen darauf hin, dass bereits stark »negativ« segregierte Quartiere eine Tendenz zur Verstärkung von Segregationsprozessen in sich tragen. Walter Siebel z.B. hebt vor allem auf die Filtereffekte des Wohnungsmarktes $a b,{ }^{9}$ wenn er die kumulativen Prozesse in "sozial belasteten « Quartieren vor dem Hintergrund einer Unterscheidung zwischen Zonen/ Räumen der Integration, der Vulnerabilität und der Ausgrenzung so beschreibt:

Zurück bleibt eine benachteiligte Bevölkerung in einem heruntergekommenen Cebiet mit mangelhafter Versorgung, einem negativen Image und negativen Nachbarschaftseffekten, die sich zu einer Kultur der Randständigkeit verdichten können. Das Gebiet ist zu einer eigenständigen Quelle von Benachteiligung geworden. In diesen Räumen intensivieren sich die Konflikte um die Integration von Zuwanderern. Fatalerweise wird nämlich gerade dort häufig darüber entschieden, ob die neu Zugewanderten an den Rand der Cesellschaft gedrängt werden oder sich integrieren können. Die Filtermechanismen auf den Wohnungsmärkten lenken die Zuwanderer in der Regel nicht

8 Die vorliegende Empirie bezieht sich vor allem auf den Grundschulbereich. Hier zeigt sich, dass die Entwicklung der wohnräumlichen Verhältnisse und das Schulwahlverhalten miteinander korrespondieren. Zum Beispiel: Eine Untersuchung des SVR-Forschungsbereichs an 108 Crundschulen in vier innerstädtischen Bezirken Berlins ergab, dass der Anteil ausländischer Schüler an jeder fünften Grundschule mehr als doppelt so hoch ist wie der Anteil ausländischer Kinder im Grundschulalter im dazugehörigen Schulbezirk (Sachverständigenrat deutscher Stiftungen für Integration und Migration: Policy Brief-Segregation an Crundschulen, Berlin, November 2012.

9 Siebel, Walter: Die Kultur der Stadt, Berlin 2015. 
in die Quartiere der integrierten einheimischen Mittelschicht, sondern in Nachbarschaften, in denen die Verlierer des ökonomischen Strukturwandels leben. Verlierer sind selten in der Lage oder auch nur willens, tolerant und offen mit Fremden umzugehen. Im Gegenteil, sie brauchen Sündenböcke, eine Rolle, für die sich Fremde immer schon besonders geeignet haben. Wenn dann in solchen erzwungenen Nachbarschaften eine heruntergekommene Umwelt ihren Bewohnern tagtäglich vor Augen führt, daß sie am Rand der Gesellschaft angekommen sind, dann ist es nicht verwunderlich, daß dies keine Orte gelingender Integration sind, sondern im Gegenteil Orte aggressiver gegenseitiger Abgrenzung. ${ }^{10}$

Es bedürfte aber einer genaueren Klärung, ob und wie dies für Quartiere der Innenstadtbezirke von Berlin zutrifft, ${ }^{11}$ die sich zugleich in einem komplizierten Prozess der Aufwertung befinden. Die immer wieder anzutreffende a-priori-Verknüpfung segregierter Quartiere mit dem Merkmal eines hohen Migrantenanteils ist kaum haltbar, wie z.B. die Entwicklung in den Großsiedlungen am Rande Berlins zeigt. Schon gar nicht ist die Gleichsetzung von hohem Migrantenanteil und belasteten Quartieren oder sozialen Brennpunkten aufrechtzuerhalten. Kaum gezweifelt werden kann an der Filterfunktion des Wohnungsmarktes. Erwartet werden muss also, dass auch viele der neu in die Stadt kommenden Geflüchteten auf ihrer Suche nach längerfristiger Bleibe und preiswertem Wohnraum in solche Quartiere einmünden. Insbesondere wäre allerdings der Frage nachzugehen, ob die sozio-ökonomischen Indikatoren, die man gemeinhin für die Beschreibung benachteiligter Bevölkerungsgruppen in Anschlag bringt, sich in der immer wieder - auch bei Siebel - angenommenen weitgehenden Deckung mit migrantischem Hintergrund bewegen.

Ob pädagogisch den innerschulischen Binneneffekten der beschriebenen Segregiertheit entgegengewirkt werden kann, hängt nicht zuletzt von den Ressourcen $a b$, die diesen Schulen zur Verfügung stehen. Von daher

10 Ebd., S. $361 f$.

11 Vgl. hierzu ausführlich Bochum, Ulrich/Butler, Jeffrey/Kohlmeyer, Klaus/Odenwald, Stephanie: Soziale Spaltungen in Berlin, Hamburg 2016, insbesondere der Abschnitt: »Soziale Spaltung durch ungleiche Bildungschancen - ungleiche Bildungschancen durch soziale Spaltung«, S. 87-114. 
kann ein Bonusprogramm, ${ }^{12}$ das die besondere Lage solcher Schulen anerkennt und sie mit zusätzlichen Mitteln versorgt, sinnvoll sein.

\section{Die Schulen stärken - aber wie?}

Das schon erwähnte SVR-Gutachten plädiert dann auch nachdrücklich dafür, die "Segregierten Schulen« in den Stand zu setzen, tatsächlich den mit diesem Typ von Schulen verbundenen Negativeffekten entgegen zu wirken und ein anregendes und förderliches, nach innen differenziertes Bildungsklima aufzubauen - und hat hierfür viele Vorschläge und Beispiele guter Praxis.

Im Berliner Kontext wäre hierzu auch die Rütli-Schule zu zählen, ${ }^{13}$ bei der zu überprüfen wäre, wie weit der Turnaround gelungen oder wie fragil er noch ist und vor allem auch, wie er erfolgte. In unserem Kontext ist dabei vor allem der Blick auf die Vorbereitung des Übergangs nach der Sekundarstufe 1 von Interesse.

Allerdings ist es fraglich, ob ein einzelschulischer Ansatz über einige gute Beispiele hinaus wirksam genug ist. Dies gilt insbesondere, wenn berücksichtigt wird, wie stark die Binnenverhältnisse in den ISSen mit ihrer sozialräumlichen engeren und weiteren Umwelt in Beziehung stehen - was sich auch an der räumlichen Konzentration der besonders "belasteten" ISSen zeigt. Zwischen Landespolitik und Einzelschule muss deshalb den Bezirken als Gestaltungs- und Koordinierungsebene Aufmerksamkeit gewidmet werden. ${ }^{14}$

Ein Vorschlag zur Zielgruppendifferenzierung aufgrund der bisherigen Erfahrungen von Berlin braucht dich!:

Gruppe 1: ist bereits auf dem Weg, hat hohe Lernbereitschaft und gelangt ohne zusätzliche Unterstützung in Ausbildung. Hier reicht ein Impuls durch die Botschaft »Berlin braucht dich!«.

12 Bonusprogramm: https://www.berlin.de/sen/bildung/schulqualitaet/bonus-programm

13 Aus den zahlreichen Publikationen zur Rütli-Schule z.B.: https://deutsches-schulportal. de/schulkultur/was-macht-die-ruetli-schule-in-berlin-heute/vom 9.7.2018.

14 Zur Grundinformation über die Rolle der Bezirke in Berlin vgl. Friedrich-Ebert-Stiftung: Kommunalpolitik verstehen im Land Berlin. Für ein junges Politikverständnis, Berlin 2010. 
Gruppe 2: ist gut in der Schule, interessiert an Ausbildung, hat keinen Zugang zu qualifizierten Ausbildungsbetrieben, kommt durch Betriebsbegegnungen mit Hilfe von Berlin braucht dich! in Ausbildung an.

Gruppe 3: weicht in ihren Leistungen und in ihrem Sozialverhalten von den Erwartungen an Auszubildende im klassischen Sinne ab und benötigt aufeinander aufbauende Betriebsbegegnungen, um schrittweise die erforderlichen Kompetenzen und Erfahrungen zu gewinnen, ist nicht so leistungsstark, verfügt aber über genügend Potenzial zur Bewältigung der Ausbildungsanforderungen, schaffen oft den Weg über Einstellungsverfahren nicht.

Gruppe 4: benötigt weiterführende Ausbildungsvorbereitung und gezielte Förderung, um in Ausbildung anzukommen, ein großes ungenutztes Potenzial, das aus der Ausbildung zurückgehalten wird dadurch, dass sie sich (noch) nicht bemüht und nicht genommen wird.

Gruppe 5: Niemand darf verlorengehen, auch Personen mit multiplen Schwierigkeiten.

Auch bei lupenreiner Vierstufigkeit wird es immer Personen geben, die nicht eine Ausbildung beginnen können, sondern einer gesonderten Vorbereitung auf Ausbildung bedürfen (Gruppen 4 und 5).

\section{Übergangsverläufe: Differenzierungen innerhalb der Schüler*innenschaft}

Es ist eine wichtige »realistische Wende«, nun davon auszugehen, dass es in den in Rede stehenden ISS (wie insgesamt an den Sekundarschulen) in Hinblick auf den (möglichen) Übergang in eine Berufsbildung innerhalb der Schüler*innenschaften erhebliche Differenzierung gibt. ${ }^{15}$ Demzufolge wird nicht mehr davon ausgegangen, dass es darum gehe, für alle Schülerinnen

15 Ein Vorschlag zur Zielgruppendifferenzierung aufgrund der bisherigen Erfahrungen von Berlin braucht dich!:

Cruppe 1: ist bereits auf dem Weg, hat hohe Lernbereitschaft und gelangt ohne zusätzliche Unterstützung in Ausbildung. Hier reicht ein Impuls durch die Botschaft »Berlin braucht dich!«.

Cruppe 2: ist gut in der Schule, interessiert an Ausbildung, hat keinen Zugang zu qualifizierten Ausbildungsbetrieben, kommt durch Betriebsbegegnungen mit Hilfe von Berlin braucht dich! in Ausbildung an. 
und Schüler der Abschlussklassen den unmittelbaren Eintritt in eine ungeförderte Berufsausbildung zu bahnen. Es ist aber die Frage, ob zu diesem Zeitpunkt schon die auf den Schultyp bezogene Zuschreibung, nach der die Berufsausbildung gewissermaßen der »natürliche« Bildungsanschluss für Absolvent*innen der Sekundarschule sei, überwunden wurde. Eine solche Zuschreibung verkennt, dass es auch in der Integrierten Sekundarschule Schülerinnen und Schüler gibt, die den Weg einer weiterführenden allgemeinen Schulbildung einschlagen (wollen) und für die der weitere Schulbesuch, den viele mit dem Wechsel in die OSZs vollziehen, keine »Verlegenheitslösung« darstellt, sondern der Versuch, einen höheren allgemeinen Bildungsabschluss zu erreichen. ${ }^{16}$

Insofern kann man vielleicht in Hinblick auf den direkten Übergang in eine Berufsausbildung drei Gruppen unterscheiden, nämlich (1) jene, die eine weiterführende allgemeine Schulbildung anstreben ${ }^{17}$ und für die Berufsaus-

Gruppe 3: weicht in ihren Leistungen und in ihrem Sozialverhalten von den Erwartungen an Auszubildende im klassischen Sinne ab und benötigt aufeinander aufbauende Betriebsbegegnungen, um schrittweise die erforderlichen Kompetenzen und Erfahrungen zu gewinnen, ist nicht so leistungsstark, verfügt aber über genügend Potenzial zur Bewältigung der Ausbildungsanforderungen, schaffen oft den Weg über Einstellungsverfahren nicht.

Gruppe 4: benötigt weiterführende Ausbildungsvorbereitung und gezielte Förderung, um in Ausbildung anzukommen, ein großes ungenutztes Potenzial, das aus der Ausbildung zurückgehalten wird dadurch, dass sie sich (noch) nicht bemüht und nicht genommen wird. Cruppe 5: Niemand darf verlorengehen, auch Personen mit multiplen Schwierigkeiten.

Auch bei lupenreiner Vierstufigkeit wird es immer Personen geben, die nicht eine Ausbildung beginnen können, sondern einer gesonderten Vorbereitung auf Ausbildung bedürfen (Gruppen 4 und 5).

16 Im Hintergrund geht es dabei auch um Prestige und Wertigkeit, die der Berufsausbildung zugemessen wird, gerade auch in den Herkunftsmilieus der Jugendlichen. Abgehandelt wird hierbei immer auch die Attraktivität von Berufsausbildung auch in dem Sinne, welche weiteren Perspektiven sie eröffnet. Vgl. hierzu u.a. Kruse, Wilfried/Strauß, Jürgen/ Braun, Frank/Müller, Matthias: Rahmenbedingungen der Weiterentwicklung des Dualen Systems beruflicher Bildung (=Arbeitspapiere Hans-Böckler-Stiftung 167), Düsseldorf 2009.

17 Wie Bildungssenatorin Scheeres mitteilte, haben sich 2015 aus den Sekundarschulen ohne gymnasiale Oberstufe im Schnitt nur 5 Schüler*innen mit Gymnasialempfehlung an einem Oberstufenzentrum angemeldet. Das Potenzial wird aber sehr viel größer eingeschätzt. Deswegen ist angestrebt, dass mehrere ISSen zusammen ein Oberstufenzentrum erhalten, um die direkten Wege zu stärken. Vieth-Entus, Susanne: Die Mischung stimmt nicht, in: Tagesspiegel vom 3.6.2015. 
bildung deswegen im Moment keine Option darstellt, ${ }^{18}(2)$ jene, die für einen direkten Übergang in eine Berufsausbildung infrage kommen, eine Gruppe, die in sich noch einmal zu differenzieren ist, und (3) jene, denen aus verschiedenen Gründen weder die erste noch die zweite Option zur Verfügung steht, deren Perspektive aber auf Berufsausbildung oder auch auf unmittelbare Erwerbstätigkeit geht. ${ }^{19}$

Wichtig ist festzuhalten, dass es sich hier um (Erfahrungs-)Werte handelt, die sich auf die Schlussphase der Sekundarschulzeit beziehen. Es wird hier also auf ein Ergebnis der Sekundarschulzeit geblickt, das sicherlich auch durch die Entscheidung zwischen Sekundarschule und Gymnasium an der Schwelle nach Klasse 6 mit beeinflusst wurde - also durch die zahlenmäßig hohe Präsenz von Schülerinnen und Schülern, die sich selbst oder denen der unmittelbare Eintritt in ein Gymnasium nicht zugetraut wurde oder denen er (sozial) nicht als angemessen erschien.

Das bedeutet aber auch, dass die skizzierte »Gruppierung « kein zwangsläufiges Resultat, sondern hergestellt ist u.a. auch vermittels der faktischen Qualität des schulischen Geschehens angesichts sehr schwieriger Umfeldbedingungen, in denen insbesondere die "Segregierten Schulen« agieren und angesichts der besonders schwierigen biografischen Phase, in der sich diese Jugendlichen befinden (Stichwort: Pubertät).

Insbesondere geht es dabei um die Umsetzung der mit der Einführung der Sekundarschule postulierten stärkeren Individualisierung im schulischen Lehr-Lern-Kontext, die auch in Hinblick auf Berufsorientierung als besonders wichtig angenommen werden kann.

\section{Über die Entstehung prekärer Übergangsverläufe}

Von daher ist es durchaus von Interesse, von »prekären Übergangsverläufen« aus auf deren Entstehung zurückzublicken. Es geht dabei um risikobehaftete Übergangsverläufe von der Schule in die Arbeitswelt. Eine vom Deutschen

18 Möglicherweise aber wird der Übergang in Berufsausbildung schon nach einem Jahr OSZ, also z.B. nach dem Erwerb des MSA, zu einer realen Option. Dieser Kreis junger Leute muss künftig stärker in den Blick genommen werden.

19 Die Jugendlichen gehören zur großen Gruppe jener, die in diversen Maßnahmen münden, oder nach Absolvieren der Schulpflicht und nach dem Erreichen des 18. Lebensjahres aus dem offiziellen Blick geraten. 
Jugendinstitut (DJI) im Auftrag der Landeshauptstadt München durchgeführte Studie, die junge Leute vier Jahr nach dem Ende ihres Schulbesuchs befragte, gibt hierfür einigen Aufschluss. ${ }^{20}$

Die Münchner Forscher*innen identifizieren »Bewältigungstypen im Übergangsprozess « und heben damit auf die Voraussetzungen und Bedingungen ab, einen biografisch riskanten Prozess zu meistern. Sie unterscheiden vier Typen:

a. Typ Normalisierung: Entschlossenes Handeln verbunden mit einer starken Orientierung auf Ausbildung und Erwerbsarbeit

b. Typ Resilienz: ${ }^{21}$ Starke Orientierung auf Ausbildung und Erwerbsarbeit trotz ausgeprägter Belastungserfahrungen

c. Typ Subsistenzorientierung: Finanzielle Unabhängigkeit als Lebensmittelpunkt mit einer fehlenden Orientierung an Ausbildung

d. Typ Perspektivlosigkeit: Zielloses und fremdstrukturiertes Übergangshandeln verbunden mit ausgeprägten Belastungserfahrungen

Betont wird, dass bei vielen der Jugendlichen, deren Bewältigungsformen in diesen Typen zusammengefasst wurden, am Übergang erhebliche zusätzliche Belastungen bestehen, die sich vielfach außerhalb des Gesichtsfelds der am Übergang beteiligten Institutionen bewegenten. Alle einbezogenen Übergangsverläufe hätten in den zurückliegenden Jahren einen »teils fragmentierten Weg in die Arbeitswelt" genommen. Es ist also wohl anzunehmen, dass auch bei den Jugendlichen, die den eher stabileren, zukunftsbezogenen Typen $\mathrm{a}$ und $\mathrm{b}$ zugeordnet worden, die Bewältigungsweise fragil bleibt.

Vor diesem Hintergrund formulieren die Autor*innen eine Reihe wichtiger Schlussfolgerungen und Handlungsempfehlungen:

- In Schule und Ausbildung müsse eine Lernumgebung geschaffen werden, in denen Jugendliche auch mit ihren lebensweltlichen Kontexten

20 Landeshauptstadt München: Prekäre Übergangsverläufe. Entstehungsbedingungen risikobehafteter Übergänge, März 2015. AutorInnen des DJl: Heike Großkurth, Tilly Lex, Nina Lichtwardt, Sylvia Müller, Frank Tillmann.

21 Resilienz (von lateinisch resilire zurückspringen, abprallen) oder psychische Widerstandsfähigkeit ist die Fähigkeit, Krisen zu bewältigen und sie durch Rückgriff auf persönliche und sozial vermittelte Ressourcen als Anlass für Entwicklungen zu nutzen. 
wahrgenommen werden und eine Sensibilität für ihre persönlichen Belastungen besteht. ${ }^{22}$

- Irritationen in den Übergangsverläufen und Ausbildungsabbrüchen wird vorgebeugt, wenn stärker als bisher auf die individuellen beruflichen Präferenzen der Jugendlichen eingegangen wird, ${ }^{23}$ weil dies eine motivationale Voraussetzung darstellt, auch schwierige Phasen durchzustehen.

- Beim Übergang von der Schule in die Arbeitswelt wird in der Regel auf direkten Anschluss gesetzt, während schulisch-akademischen Bildungswegen oft mehr Zeit und mehr Freiraum eingeräumt wird. Viele Jugendliche benötigen aber Zeit, um Entscheidungsfähigkeit auszubilden und befriedigende Entscheidungen treffen zu können. Moratorien sinnstiftender Tätigkeiten können dabei durchaus von Vorteil sein.

- Eine Übergangsbegleitung, die bereits in der Schulzeit einsetzt, und in der Ausbildung fortgesetzt wird, kann wichtige stabilisierende Effekte haben.

- Bei Berichten über Unterstützung in krisenhaften Situationen kommen institutionelle Unterstützungsakteure kaum vor. Hier müsste also mehr Transparenz, Nähe und niedrigschwellige Zugänglichkeit geschaffen werden, ohne die jungen Erwachsenen mit Betreuung zu bedrängen. Freiwilligkeit bleibt ein Grundprinzip. Peer-Ansätze haben sich oftmals als gut erwiesen.

- Neuorientierungen bei eingetretenen besonderen Belastungssituationen und Brüchen benötigen - meist sozialpädagogische - Unterstützung. Dies gilt auch für einen motivationalen Neuaufbau, der beim Typ »Perspektivlosigkeit« erforderlich ist.

22 Stichwort Lebensweltorientierte Schule, vgl. hierzu Künzel, Manfred: »Ein Netz von Entwicklungsräumen. Wege zu einer lebensweltorientierten Schule«, in: Thomas, Peter Martin/Calmbach, Marc (Hg.): Jugendliche Lebenswelten. Perspektiven für Politik, Pädagogik und Gesellschaft, Heidelberg 2013, S. $137 \mathrm{ff}$.

23 Hier schließt das Konzept der berufsfeldbezogenen Neigungsgruppen an, das von Berlin braucht dich! immer wieder ins Gespräch gebracht wurde. 


\section{Weichenstellungen für prekäre Übergangsverläufe}

Es ist anzunehmen, dass die Dispositionen für prekäre Übergangsverläufe schon frühzeitig gelegt werden, insbesondere in den letzten Jahren der Sekundarschulzeit. Wie könnten sie beschrieben werden? Ursula Beicht und Mona Granato beschreiben den Kontext, in dem sich vor allen jene Jugendlichen mit Migrationsgeschichte bewegen, die zugleich unter belastenden Verhältnissen von Armut aufwachsen, so:

Das Auseinanderdriften der sozialen Lebenslagen von Familien in Deutschland, wo bereits jedes sechste Kind am Rande der Armutsgrenze lebt, ist längst gesellschaftliche Realität. Kinder und Jugendliche mit Migrationshintergrund wachsen häufiger als diejenigen ohne Migrationshintergrund in $\mathrm{Fa}$ milien auf, die durch sozioökonomisch schwierige Lebenslagen geprägt sind. Migrantenfamilien leben doppelt so oft an der Armutsgrenze wie Familien ohne Migrationshintergrund. ${ }^{24}$

Allerdings weisen sie ausdrücklich darauf hin, dass Einkommensarmut nur ein Aspekt ist, weil Armut sich in allen Lebensbereichen niederschlägt, und damit die Chancen auf eine gleichberechtigte gesellschaftliche Teilhabe einschränkt. Aber: Auch in der migrantischen Bevölkerung differenzieren sich die Lebenslagen aus, und Lebenslagen beeinflussen, aber determinieren nicht, wie Jugendliche sich entwickeln und sich selbst und die Gesellschaft, in der sie leben, sehen.

Insgesamt ist eine "Pluralisierung der Lebenswelten und Wertorientierungen junger Menschen mit und ohne Migrationshintergrund « $\mathrm{zu}$ beobachten. »Die Differenzierungslinien verlaufen dabei unterschiedlich; zum Teil zwischen Jugendlichen mit Migrationshintergrund oder zwischen Mädchen und Jungen, seltener hingegen zwischen den Gesamtgruppen der Jugendlichen mit bzw. ohne Migrationshintergrund. [...] Junge Frauen bzw. Männer mit einem Migrationshintergrund haben von ihren Orientierungen und ihrem jugendkulturellen Stil und Gemeinschaftsbildung her genauso

24 Beicht, Ursula/Granato, Mona: Prekäre Übergänge vermeiden - Potenziale nutzen. Junge Frauen und Männer mit Migrationshintergrund an der Schwelle von der Schule zur Ausbildung (=WISO Diskurs), Bonn 2011, S. 11. 
wenig gemeinsame Präferenzen und homogene Orientierungen wie weibliche und männliche Jugendliche ohne Migrationshintergrund «. ${ }^{25}$

Aber, und das ist für unseren Zusammenhang sehr entscheidend, "schließt dies nicht aus, dass sie in Bildung und Beruf unabhängig von ihren Orientierungen und Präferenzen soziale Grenzziehungen erfahren und auf diese Weise auf eine als sethnisch definierte Zugehörigkeit verwiesen werden - auch wenn sie sich selbst anders definieren. ${ }^{26}$

Zugleich ist der Übergang von der Schule in Berufsausbildung bzw. Arbeitswelt oder weiterführende Bildung insgesamt unübersichtlicher und schwieriger geworden. Das liegt zum einen daran, weil »Normalbiografien«, an denen man sich orientieren könnte oder möchte, kaum noch zur Verfügung stehen, zum anderen aber daran, weil z.B. in vielen Ausbildungsberufen die Anforderungen erheblich gestiegen und Einstiege in Ausbildung vielfach schwierig bleiben.

Beicht und Granato formulieren vor diesem Hintergrund eine für unseren Zusammenhang wichtige Schlussfolgerung: »Diese Veränderungen sind mit höheren Anforderungen an die Jugendlichen verbunden. Sie benötigen eine höhere Eigenverantwortung, ein größeres Engagement, mehr Durchhaltevermögen und Frustrationstoleranz, um diese Statuspassage zu gestalten und erfolgreich zu bewältigen. ${ }^{27}$

Je belasteter die Gesamtsituation ist und je schwieriger es erscheint oder eingeschätzt wird, die Vorstellungen über das eigene künftige Leben zu realisieren, desto mehr muss an Eigenverantwortung, Engagement, Durchhaltevermögen und Frustrationstoleranz aufgebracht werden. Die Jugendlichen müssen also eine erhebliche Kraft aufbringen, ${ }^{28}$ um nicht zu resig-

25 Ebd.

26 Ebd., S. $11 f$.

27 Ebd., S. 12.

28 Und dies in einer biografischen Periode, in der die Identitätsfindung von zentraler Bedeutung ist. An den »segregierten «Schulen, auf die hier besondere Aufmerksamkeit gerichtet wird, finden sich viele Jugendliche aus muslimischen Milieus. Für sie ist die Frage, wie sie sich dem Islam zuordnen, eine sehr wichtige Identitätsproblematik der Jugendphase und kann durchaus andere Fragen und Aufgaben überlagern. Eine an biografischen Entwicklungsprozessen orientierte Typologie junger Muslime liefert eine Studie von Wensierski, Hans-Jürgen/Lübcke, Claudia: »Als Moslem fühlt man sich hier auch zu Hause«. Biographien und Alltagskulturen junger Muslime in Deutschland, Opladen/Berlin/Toronto 2012. 
nieren und z.B. die Schule als eine Übung zu empfinden, die ihnen nichts nützt. Unter solchen Umständen sind Motivationen sehr fragil und können schon bei kleineren Enttäuschungen zusammen brechen. ${ }^{29} \mathrm{Wie}$ also können Jugendliche in einer solchen Lage »Kraft tanken«?

\section{Praktisch-betriebliche Berufsorientierung: ein Fenster zum Leben außerhalb der Schule}

Nach Lage der Dinge kann die Schule diese Aufgabe wohl nicht allein - auch dann nicht, wenn sie pädagogisch innovativ eingestellt ist - erfüllen, sondern braucht Partner. Wie Schulen also in die Welt ihrer Schülerinnen und Schüler eingebettet sind, ist dort, wo sich eine Schülerschaft mit Migrationshintergründen konzentriert, integrationspolitisch von erheblicher Bedeutung.

Insofern ordnet sich eine Berufsorientierung, die über eine Abfolge von Betriebsbegegnungen erfolgt - wie dies z.B. bei Berlin braucht dich! geschieht -, in jene Konzepte ein, die den traditionellen schulischen Lerntyp durch die praktische Öffnung der Schule zum Leben außerhalb der Schule zu ergänzen, im besten Fall zu reformieren suchen. Natürlich macht den besonderen Stellenwert einer solchen, auf Betriebsbegegnungen aufbauenden Berufsorientierung auch ihr evidenter Zukunftsbezug aus.

29 In der schulpsychologischen Forschung wird davon ausgegangen, dass sich an sozial besonders belasteten Schulen viele sogenannte »schwierigen Schüler«befinden. In Hinblick auf das Unterrichtsgeschehen und das Verhalten in der Schule werden z.B. sogenannte »schwierige Schüler« so unterschieden: Verhaltensauffällige Schüler fallen auf durch Unhöflichkeiten, Regelverletzungen und Nachlässigkeiten; Schüler mit Lernstörungen - dazu gehören Lernhemmungen und -behinderungen in unterschiedlichen Bereichen, Schüler mit ADHS - sind unaufmerksam, impulsiv und hyperaktiv, emotional schwierige Schüler oftmals traumatisierte Kinder und Jugendliche, die unter Ängsten und Minderwertigkeitsproblemen leiden und bei denen aggressive oder antisoziale Impulse zu beobachten sind; Schüler mit sozialen Störungen - fallen auf durch Misstrauen und fehlende Empathie, zeigen sich bindungslos und unfähig, sich einzuordnen. Vgl.: Czerwenka, Kurt: Umgang mit schwierigen Schülern. Erkenntnisse aus der Verhaltenspsychologie Vortrag vor der Konferenz Starke Schule am 4. Mai 2009 in Berlin, zusammengefasst in: Bildungsportal NRW des Ministeriums für Schule und Weiterbildung des Landes Nordrhein-Westfalen unter der Überschrift »]eder siebte Schüler gilt als schwierig« www.schulministerium. nrw.de, aufgerufen am 17.1.2016). 
Gute Berufsorientierung könnte deshalb für die Sekundarschulen vor allem in den abschließenden Schuljahren eine erhebliche fokussierende pädagogische Kraft erzeugen. ${ }^{30}$ Allerdings gilt dies - je näher des Ende der Schule rückt verstärkt - nur dann, wenn es realistische Aussichten auf einen Einstieg in Ausbildung gibt und die Berufswünsche der Jugendlichen, die ja im Zuge des mehrjährigen Berufsorientierungs-Prozesses schon einem erheblichen Realitätscheck ausgesetzt waren, nicht völlig frustriert werden. An den meisten Integrierten Sekundarschulen - auch an jenen, die zum Konsortium von Berlin braucht dich! gehören - ist der pädagogische Stellenwert der Berufsorientierung im Sinne der Qualifizierten Vierstufigkeit aber noch nicht (ausreichend) geklärt.

Bislang wird der schulische Umgang mit den Betriebsbegegnungen vor allem in ihrer Vor- und Nachbereitung gesehen. Genauer betrachtet, erweist sich demnach die Rede von der »Vor- und Nachbereitung« bereits als eine zu starke konventionelle Einschränkung. ${ }^{31}$ Viel genereller stellt sich die Frage, welchen pädagogischen Stellenwert Betriebsbegegnungen/Betriebspraktika im Rahmen einer über die verschiedenen Schuljahre fortschreitenden Berufsorientierung haben. Trotz der Einführung von dualem Lernen erscheint dies noch zu wenig wirklich geklärt, insbesondere, was die Einbettung von Betriebsbegegnungen in eine integrative pädagogische Praxis der Berufsorientierung betrifft.

\section{Zur "Kunst" der Einbettung von Praktika in schulische Berufsorientierung}

Praxis/Praktikum wird häufig noch als eine Art Ausflug in eine andere Welt erlebt und als gewissermaßen "schulpädagogisch freier Raum« verstanden. Demgegenüber betreibt Berlin braucht dich! eine massive Aufwertung

30 Das ist der Sinn von der Rede der »Berufsorientierung als Aufgabe der ganzen Schule«. Vgl. hierzu u.a. sehr früh: Famulla, Gerd-Ewald/Butz, Bert/Deeken, Sven/Michaelis, Ute/ Möhle, Volker/Schäfer, Birgit: Berufsorientierung als Prozess. Persönlichkeit fördern, Schule entwickeln, Übergang sichern. Ergebnisse aus dem Programm sSchule-Wirtschaft/Arbeitsleben<, Hohengehren 2008.

31 Die folgenden Überlegungen wurden in einer Expertise für BQN im Jahr 2013 entwickelt: Kruse, Wilfried: Das »System« der Betriebsbegegnungen von Berlin braucht dich! und die Rolle der Schulen, unveröffentlichtes Manuskript Berlin/Dortmund April 2013. 
der Betriebsbegegnungen, die ihren Sinn natürlich nur dann ergibt, wenn diese nicht auf vier Schuljahre verteilte »Erfahrungsinseln« bleiben, sondern lebendige Elemente eines fortschreitenden individuellen Prozesses der Auseinandersetzung mit der eigenen nachschulischen Zukunft sind.

Es ist also der mit Vierstufigkeit noch zu mechanisch ausgedrückte Aufbau individueller Prozesse zunehmender Entscheidungsfähigkeit in Hinblick auf die eigene berufliche Zukunft, um den es bei der schulischen Berufsorientierung vor allem geht oder gehen müsste. Attraktive Betriebsbegegnungen, die "aufgabenbezogen" aufeinander aufbauen, sind dabei ein unverzichtbarer Bestandteil.

In den Blick rücken so vor allem die individuellen berufsorientierenden Bildungsprozesse. Die »Kunst« der Schule bestünde darin, für alle Schülerinnen und Schüler ab Klasse 7 solche kontinuierlichen Bildungsprozesse zu sichern, in denen Jahr um Jahr Betriebsbegegnungen organisatorisch und pädagogisch gut eingebettet sind. Dies ist im Übrigen auch eine zentrale Voraussetzung dafür, dass die Berufsausbildung Nachteile ausgleichend wirken kann: "Wenn es gelingt, eine die gesamte Schulzeit begleitende berufsorientierende und berufsvorbereitende Bildung in den allgemeinbildenden Schulen zu etablieren, dann ist zu erwarten, dass sich die kompensatorische Funktion der beruflichen Bildung entfalten kann. ${ }^{32}$

Vor diesem Hintergrund wird klar, dass es auch aus integrationspolitischen Gründen darum geht, für alle Schülerinnen und Schüler im Rahmen der schulischen Berufsorientierung eine Abfolge von Betriebsbegegnungen/ Betriebspraktika sicher erreichbar zu machen oder vorzuhalten, die in einem pragmatischen Sinne Qualitätsmindeststandards aufweisen. Insofern kann man aus dem Postulat gleichwertiger Zugänge zu Bildung geradezu einen Anspruch aller Schülerinnen und Schüler auf gute Betriebsbegegnungen ableiten.

Ins Zentrum des schulischen Umgangs mit Betriebsbegegnungen/Praktika stehen - so kann resümiert werden - vier wichtige Aufgaben:

Erstens: Die Prüfung der Gesamtheit der Praktikumsplätze/Betriebsbegegnungsplätze, auf die die Schule bisher zurückgreift. Dies meint alle Plätze, die z.B. im vorangegangenen Jahr genutzt wurden, unabhängig davon, wer sie eingebracht hat, also auch jener, die sich Schüler*innen selbst ge-

32 Rauner, Felix/Piening, Dorothea: Umgang mit Heterogenität in der beruflichen Bildung. Eine Handreichung des Projekts KOMET, Bremen 2010. 
sucht haben. Entscheidend ist hierbei, welche Berufsfelder, welche Betriebstypen und welche Qualitäten vertreten sind, und die Frage, ob das Gesamtangebot in Hinblick auf Berufsfelder und Qualität ergänzt bzw. erweitert werden muss, ggf. auch aus dem Potenzial der "vorhandenen« Betriebe heraus (z.B. Ausweitung der angebotenen Formate von Betriebsbegegnungen). Dies muss natürlich immer in Beziehung zu dem differenzierten Profil der Schüler"innenschaft betrachtet werden.

Wenn die Notwendigkeit von Ergänzungen und Erweiterungen gesehen wird, folgt daraus, dass gezielt weitere Betriebe eingeworben werden müssen. Insgesamt muss stets darauf geachtet werden, dass sich in der Gesamtheit der Praktika mehrheitlich Berufsfelder und Betriebe befinden, in denen aus dem Kreis dieser Schüler*innen heraus eine Ausbildung möglich ist und realistisch ist. Zumindest die Praktikumsplätze in der 9. Jahrgangsstufe sollten in beruflichen Feldern stattfinden, zu deren Ausbildung der Zugang für diese Schülerinnen und Schüler nicht vollständig oder doch mehrheitlich irreal ist. Damit ist nicht der Erwartungen an »Klebeeffekte« das Wort geredet, sondern dem Erfordernis, die fragilen Motivationen weiter aufzubauen und nicht zu gefährden.

Zweitens: Wo noch nicht vorhanden, geht es um den Aufbau eines stabilen Netzwerks mit jenen Betrieben, auf die von Seiten der Schule bei Prakti$\mathrm{ka} /$ Betriebsbegegnungen zurückgegriffen wird. Bisher sind diese Kontakte meist nur punktuell; sie müssen aber verstetigt werden, um mit den Betrieben gemeinsam eine Qualitätsentwicklung in Hinblick auf Praktika/Betriebsbegegnungen einzuleiten, ohne die Betriebe dabei zu überfordern.

Drittens: Im Sinne einer guten Berufsorientierungs-Pädagogik muss die Aufmerksamkeit der Schule, respektive der zuständigen Lehrer"innen sich in besonderer Weise auf jene Schüler*innen richten, deren persönliche Dispositionen fragil sind und die deshalb in besonderer Weise Erfolgserlebnisse und Bestätigung benötigen, und kritisch auf jene Betriebsbegegnungen/ Praktika, die in ihrer (aktuellen) Qualität gerade noch tolerabel, aber problematisch sind. Besonders ungünstig - aber bisher eher die Regel als die Ausnahme - ist es, wenn in Hinblick auf die Beruforientierung besonders fragile Schüler*innen auf diese Betriebe treffen. Von daher gehört zur schulischen Berufsorientierungs-Gestaltung auch, eine pädagogisch sinnvolle Verteilung der Praktikums/Betriebsbegegnungsplätze anzustreben.

Viertens: Gerade vor dem Hintergrund der Differenziertheit der Profile der Schülerinnen und Schüler in den letzten Klassen der Sekundarschu- 
le insbesondere auch in Hinblick auf ihre Chancen auf einen betrieblichen Ausbildungsplatz, ist es besonders wichtig, alle an der Schule vorhandenen Praxisansätze beruflicher Orientierung, wie z.B. "Produktives Lernen", "Produktionsschulen", "Servicelernen" mit den Betriebsbegegnungen im Rahmen der Qualifizierten Vierstufigkeit wirksam zu kombinieren. Eine solche pädagogisch durchdachte Kombination der verschiedenen Formen des Praxislernens im Rahmen einer weit gefassten schulischen Berufsorientierung ist auch integrationspolitisch bedeutsam. Sie vermeidet Diskriminierungen, führt zu kumulativen Anregungseffekten und bereitet zugleich die unterschiedlichen Optionen nach Beendigung der Sekundarschule vor.

\section{Fazit}

Im Zentrum des vorangehenden Kapitels 5 steht die für das integrationspolitische Leitprojekt beunruhigende empirische Erkenntnis, dass es trotz stark verbesserter Berufsorientierung und bei hoher Motivation der Schüler"innen aus den im Konsortium mitarbeitenden Schulen diesen immer noch sehr selten gelingt, in den im Konsortium mitarbeitenden Betrieben einen Ausbildungsvertrag zu erhalten. Offenbar gibt es Benachteiligungen, die hartnäckiger sind, als dies erwartet wurde. Für die weitere Arbeit in diesem Feld ist es wichtig, besser zu verstehen, woran dies liegen könnte. Deshalb wurde in diesem Kapitel nun ein Schritt zurück gemacht zu Ansätzen und Ergebnissen aus der Forschung, die Aufklärung über die Gründe versprechen, warum der Eintritt in eine Ausbildung für eine beträchtliche Gruppe von jungen Menschen eine starke Barriere darstellt.

Forschungen zu Prozessen sozialer und sozial-räumlicher Trennung (Segregation) bieten an, Schulen auf die Abweichung der sozialen Zusammensetzung des Schulbesuchs von der gesamtstädtischen Bevölkerungsstruktur hin zu befragen. Im Falle der am Konsortium beteiligten Integrierten Sekundarschulen sind die Anteile von Jugendlichen aus Familien mit Migrationsgeschichte und zugleich im SGB-II-Bezug überproportional hoch und z.T. stark mehrheitlich, oftmals zudem mit einer Tendenz zur weiteren Verstärkung dieses Anteils. Solche kumulativen Effekte sind auch für sozial belastete Quartiere zu beobachten, so dass auch die Beziehung zwischen Schulen und den Herkunftsquartieren ihrer Schülerinnen und Schüler stärker in den Blick genommen werden muss. 
Bedeutsam ist nun die Frage, welches die Binneneffekte von Segregiertheit im pädagogischen Raum der Schule sind - und wie Schulen damit pädagogisch produktiv umgehen können. Hier setzen z.B. Turnaround- oder »Brennpunktschul«-Konzepte an. Um hier mehr Aufschluss zu erhalten, lohnt ein Blick auf Studien zur Binnendifferenzierung von Schüler*innenschaften, z.B. hinsichtlich ihrer Übergangsverläufe von der Schule in Berufsausbildung und Arbeitswelt. Denn weder das Verhalten und die Lernhaltung noch die Verläufe sind an diesen Schulen uniform.

Die konsultierten jugendsoziologischen Studien kommen zu einem ähnlichen Ergebnis: Jugendliche in prekären Lebensverhältnissen und in segregierten Milieus mit starken schulischen und beruflichen Misserfolgserfahrungen brauchen eine erhebliche Kraft, um schon bei kleineren Rückschlägen und Misserfolgen nicht zu resignieren, Frustrationen aushalten zu können und Durchhaltevermögen zu entwickeln. In diesem Sinne kann man von einer erhöhten persönlichen Verletzlichkeit und dem Risiko einer größeren subjektiven Brüchigkeit eingeschlagener Wege ausgehen.

Schulen haben in dieser Situation also die überaus schwierige Aufgabe, Schülerinnen und Schüler darin zu unterstützen, im positiven Sinne »ihren Weg« zu gehen. Schulen können dies nicht allein, sondern brauchen hierzu außerschulische Partner. Wie Schulen in die Welt ihrer Schülerinnen und Schüler eingebettet sind, so eine der Schlussfolgerungen, ist dort, wo sich Migrationshintergründe konzentrieren, integrationspolitisch von erheblicher Bedeutung - und müsste als Aspekt ebenfalls in die querschnittspolitische Vernetzung einbegriffen sein.

Vor diesem Hintergrund wurde diskutiert, ob und wie Berufsorientierung, wie sie im integrationspolitischen Leitprojekt Berlin braucht dich! entwickelt wurde, hierfür einen wichtigen Beitrag leisten kann. Die Abfolge von Betriebsbegegnungen macht - das hat für sich genommen schon einen Stellenwert - ein Fenster zu einer wichtigen gesellschaftlichen Wirklichkeit, nämlich dem Arbeitsleben, auf. Es hängt von der Qualität der Betriebsbegegnungen $a b$, ob hieraus eine Motivation entstehen kann, sich bewusst und intensiv mit der Option einer Berufsausbildung auseinanderzusetzen. Aber Betriebsbegegnungen bleiben punktuelle Ereignisse, wenn sie nicht in eine systematische und individualisierte schulische Berufsorientierung eingebettet sind und lebensweltlich gestützt oder jedenfalls nicht unterlaufen werden (Stichwort: Quartiers- und Elternarbeit). 
Berufsorientierung in diesem Sinne und auf einem definierten Mindestniveau von Qualität aber müsste allen Schülerinnen und Schülern zur Verfügung stehen. Dafür reichen aber die Kontingente an Betriebsbegegnungen, die über Berlin braucht dich! in die mitarbeitenden Schulen kommen, nicht aus. Für das Gesamtangebot muss auf weitere Betriebe zurückgegriffen werden. Geklärt werden muss also, welche Hebelwirkung Schule für Schule und darüber hinaus mit dem Berlin braucht dich!-Kontingent erzielt werden kann. Dieser Faden wird im Kapitel 8 wiederaufgenommen.

Die Schulen sind also erneut im Fokus, aber keineswegs allein. Im nächsten Kapitel wird deshalb erneut der Blick auf die Betriebe gerichtet. 



\section{Kapitel 7}

\section{Kann beruflich-betriebliche Bildung die Benachteiligung ausgleichen?}

In Hinblick auf die integrationspolitisch wichtige Frage danach, wie Risiken im Übergang abgebaut und für alle Jugendlichen gelingende Übergänge in ein selbständiges und selbstbestimmtes Leben gebahnt werden können, kommt - so die zentrale These - der Berufsausbildung tatsächlich eine besonders wichtige Funktion zu. ${ }^{1}$ Die potenziell kompensatorische Funktion, die Berufsausbildung einnehmen kann, hängt mit dem dort vorherrschenden Lernen in der betrieblichen Praxis zusammen. Dies leistet aber Berufsausbildung nicht schon a priori, sondern nur dann, wenn sie so gestaltet wird, dass auch Jugendliche mit ungünstigeren Startvoraussetzungen in ihr wachsen können, und zwar im Regelsystem und im Rahmen heterogener Auszubildendengruppen.

In den bisherigen Diskursen dominierte aber der Blick auf die Defizite, die bestimmte Gruppen von Jugendlichen in Hinblick auf die sogenannte "Ausbildungsreife « aufweisen sollen. ${ }^{2}$ Seit einigen Jahren kommt außerdem die Auswahlpraxis der Betriebe als Hinderung stärker in den Blick. ${ }^{3}$

1 Das Kapitel 7 folgt-ebenso wie das Kapitel 6-in der Argumentation Kruse, Wilfried: Übergänge von der Schule in die Arbeitswelt in Berlin. Integrationspolitische Cestaltungsaufgaben für die Periode 2016 folgende und die Rolle von Berlin braucht dich!. Expertise für den Beauftragten des Berliner Senats für Integration und Migration, unveröffentlichtes Manuskript, Dortmund 2016.

2 Vgl. u.a. Dobischat, Rolf/Kühnlein, Gertrud/Schurgatz, Robert: Ausbildungsreife. Arbeitspapier 189 der Hans-Böckler-Stiftung, Düsseldorf 2012.

3 Zur Präsenz junger Menschen mit Migrationsgeschichte in betrieblicher Ausbildung vgl. auch: Enggruber, Ruth/Rützel, Josef: Berufsausbildung junger Menschen mit Migrationshintergrund. Eine repräsentative Befragung von Betrieben, Cütersloh 2014. 


\section{Zugangsbarrieren und Scheiternsrisiken}

Erst vor Kurzem sind Stand der Forschung, Kontroversen und Forschungsbedarf zu »Diskriminierung migrantischer Jugendlicher in der beruflichen Bildung « in einem Sammelband zusammengefasst worden. ${ }^{4}$ Empirisch zeigen sich vier Felder von Benachteiligung im Vergleich zu Jugendlichen ohne Migrationsgeschichte. Diese sind ein niedrigerer Anteil an der Gesamtzahl der Auszubildenden, eine geringere Erfolgsquote bei Bewerbungen, ein wesentlich niedrigerer Grad, den Wunschberuf zu erreichen, bei einem gleichzeitig höheren Grad der Einmündung in Ausbildungen für Berufe, die aufgrund ihrer Arbeitsbedingungen und ihrer Bezahlung als weniger attraktiv gelten. Das alles ist nicht neu. Im Zentrum des Sammelbands steht deshalb die Frage, warum dies so ist. Die These ist, dass es sich nicht um Nebenfolgen schulischer Benachteiligungen handele, sondern um eine eigenständige Form von Diskriminierung.

Die Befunde dieser Studie zur Diskriminierung beim Zugang zu Ausbildung können in sechs Punkten zusammengefasst werden: ${ }^{5}$

1. Die Annahme einer strikt leistungsgerechten Auswahl von Bewerber*innen trifft nicht zu. »In Auswahlverfahren gehen aufgrund der Unvollständigkeit relevanter Informationen vielmehr gruppenbezogene Zuschreibungen als Kalküle bezüglich der typisch zu erwartenden Leistungsfähigkeit ein, welche die Unsicherheit der Entscheidungen reduzieren.«

2. Betriebliche Rationalität, Markt und auch erwartete Personalengpässe wirken einer diskriminierenden Auswahl nicht a priori entgegen.

3. Auswahlentscheidungen werden auch unter dem Aspekt der Rechtfertigbarkeit im sozialen Umfeld getroffen, also vor allem hinsichtlich der Kunden und der Mitarbeiter*innen, aber - in kleineren Firmen - auch der Verwandten und Bekannten.

4 Scherr, Albert (Hg.): Diskriminierung migrantischer Jugendlicher in der beruflichen Bildung. Stand der Forschung, Kontroversen, Forschungsbedarf, Weinheim 2015.

5 Scherr, Albert/Janz, Caroline/Müller, Stefan: »Einleitung: Ausmaß, Formen und Ursachen der Diskriminierung migrantischer Bewerber/innen um Ausbildungsplätze«, in: Albert Scherr (Hg.): Diskriminierung migrantischer Jugendlicher in der beruflichen Bildung. Stand der Forschung, Kontroversen, Forschungsbedarf, Weinheim 2015. S. 9-32. 
4. Soziale Netzwerke spielen bei der Vergabe von Ausbildungsplätzen eine erhebliche Rolle, was bei einer mehrheitsgesellschaftlichen Prägung eher zur Privilegierung von Kandidat*innen mit deutscher Herkunft führe.

5. Gehe es vor allem um "soziale Passung«, für die ein deutliches Indiz sei, »dass zahlreiche Betriebe ihre Entscheidung für eine/n Bewerber/ eine Bewerberin letztlich an den Eindruck knüpfen, den diese/dieser bei einem Probearbeiten hinterlässt«.

6. Demgegenüber und mit Hilfe von »eigenen Kompetenzmessungen und Wahrnehmungen auf Dimensionen, die durch die schulischen Qualifikationen offensichtlich nur sehr begrenzt abgebildet werden« verliert der Prognosewert schulischer Leistungen an Bedeutung.

Tatsächlich bleiben diese Befunde hinsichtlich ihrer Erklärungskraft für die fortbestehende Benachteiligung von Jugendlichen mit Migrationsgeschichte beim Zugang zur Berufsausbildung allerdings unbefriedigend, weil ohne genaue Klärung weiter mit der Annahme gearbeitet wird, die ausgrenzende Einstellungspraxis der Betriebe gründe vor allem im Migrationshintergrund der Bewerber*innen. Auch Scherr u.a. haben hier Zweifel:

In der gesellschaftlichen Realität und auch in der Wahrnehmung der Betriebe ist die Unterscheidung von Migrant*innen und Nicht-Migrant*innen mit den Unterscheidungen nach schulischem Qualifikationsniveau verschränkt. Damit sind migrantische Bewerber*innen überproportional auch von Vorbehalten bezüglich der Eignung von Hauptschüler*innen für die betriebliche Bildung betroffen. Eine Forschung, die aufzeigt, ob und gegebenenfalls wie ethno-nationale, religionsbezogene und rassistische Diskriminierung durch eigenständige Vorurteile gegenüber den sogenannten Bildungsfernen überlagert und verstärkt werden, ist aktuell ebenso wenig verfügbar wie eine Forschung dazu, ob migrationsbezogene Zuschreibungen auch bei höher Cebildeten in gleicher Weise greifen oder gegebenenfalls durch den Bildungsstatus relativiert werden.

Besonders wichtig ist im hier aufgerufenen Zusammenhang, die potenziell kompensatorische Funktion der beruflichen Bildung ins Auge zu fassen, die aber daran geknüpft ist, dass die Jugendlichen überhaupt in Ausbildung kommen. Als eine starke Vermutung kann deshalb auch unter Berücksichtigung der gerade skizzierten Befunde formuliert werden, dass Ausbildungs- 
betriebe bei ihren Auswahlentscheidungen die Einstellung jener junger Erwachsener zu vermeiden suchen, denen hohe Scheiternsrisiken bei der Ausbildung zugeschrieben werden.

\section{Ausbildungsqualität ausschlaggebend}

Was kann unter der »kompensatorischen Funktion« der beruflichen Bildung verstanden werden? Gemeint ist damit, dass Startunterschiede zwischen verschiedenen Auszubildenden während der Berufsausbildung abgebaut werden und »vor allem Schüler, die die allgemeinbildende Schule mit schwachen Leistungen abschließen, während ihrer Berufsausbildung oft unerwartet gute Ausbildungsergebnisse erreichen.«Denn:»Der Wechsel von schulischen Lernformen zum `Lernen im Arbeitsprozess`, dem Herzstück der beruflichen Entwicklung, wirkt sich bei sschulmüden Jugendlichen oft wie eine >Befreiung« aus«. ${ }^{6}$

Das Wirksamwerden der kompensatorischen Funktion der beruflichen Bildung - und damit ihre mögliche Rolle beim Abbau von Bildungsbenachteiligung - ist jedoch kein Selbstläufer. ${ }^{7}$ Es ist von der Gestaltung der beruflichen Lernprozesse abhängig und hat umso mehr Chancen, je mehr diese an in der Schulzeit geschaffenen Voraussetzungen anknüpfen kann.

Für das Bemühen um die Sicherung und Stärkung des pädagogischen Potenzials betrieblicher Ausbildung gibt es vielfältige Anknüpfungspunkte. So sind z.B. die Gestaltungsgesichtspunkte und Konzepte, wie sie für die Praktika in Berlin braucht dich! entwickelt worden sind, ${ }^{8}$ auch für die Ausbildung nutzbar ebenso wie diverse innovative Ansätze, die in Ausbildungsbetrieben und in überbetrieblichen Ausbildungsstätten anzutreffen sind. Nicht zuletzt bietet sich auch eine Verarbeitung der Ergebnisse aus diversen

6 Piening, Dorothea/Rauner, Felix: Umgang mit Heterogenität, S. 15.

7 Vgl. hierzu auch die Broschüre: Der Beauftragte des Berliner Senats für Integration und Migration (Hg.): Alle mit dabei? Potenziale der Berufsausbildung für Jugendliche aus Einwandererfamilien, Berlin 2019.

8 Siehe das 2015 vollständig überarbeitete Handbuch Betriebsbegegnungen von Berlin braucht dich! https://www.berlin-braucht-dich.de/fileadmin/user_upload/PDFs/Handbuch_Betriebsbegegnungen.pdf 
Modellversuchen und Praxisevaluierungen an, wie sie z.B. vom Bundesinstitut für Berufsbildung gefördert oder durchgeführt wurden. ${ }^{9}$

Besonders interessant sind in diesem Zusammenhang neuere fachliche Ansätze, die die Förderung jener Jugendlicher, die bislang keinen Zugang zur betrieblichen Ausbildung hatten, nicht mehr in außerbetrieblicher Ausbildung verlagern, sondern unter dem Stichwort »Inklusion« zum Regelbestandteil der dualen Berufsausbildung machen wollen. ${ }^{10}$ Hierfür werden auch neue Kooperationsansätze zwischen Betrieben und Trägern der Jugendsozialarbeit erprobt. ${ }^{11}$

Entscheidend sei, so Petra Lippegans-Grünau, ${ }^{12}$ die Wendung auf eine Subjektorientierung in der beruflichen Bildung. Damit wird Individualisierung zu einer »regulativen Idee« der Berufsausbildung, und zwar auf Grund der Erkenntnis, »dass Lern- und Entwicklungsprozesse unterschiedlich verlaufen und pädagogische Prozesse deshalb differenziert angelegt werden müssen.« Vor diesem Hintergrund werden vier grundlegende Gestaltungsprinzipien für die betriebliche Berufsausbildung herausgestellt, nämlich (1) eine ganzheitliche Sicht auf die Auszubildenden, (2) ein Kompetenzansatz, (3) Individualisierung (im sozialen Kontext) und (4) Partizipation.

Diese Hinweise unterstreichen, dass es zu eng ist, den Blick nur auf die ausbildenden Betriebe $\mathrm{zu}$ richten, wenngleich diese aufgrund der wirtschaftlich-rechtlichen Verfasstheit des »dualen Systems« dominieren. Insbesondere darf es nicht zu einer Form der Betriebsfixiertheit kommen, die den zweiten zentralen Lernort, die Teilzeitberufsschule, nahezu ausblendet.

9 Vgl. hierzu die Datenbanken des BIBB, das CoodPracticeCenter (CBC) und vielfältige andere Dokumente, die insbesondere im Kontext der Initiativen zur beruflichen Benachteiligtenförderung in den Jahren 2002 bis2006 entstanden sind: https://www.bibb.de/

10 Vgl. hierzu: Severing, Eckart/Weiß, Reinhold (Hg.): Individuelle Förderung in heterogenen Cruppen in der Berufsausbildung. Befunde-Konzepte-Forschungsbedarf, Bielefeld 2014.

11 Auf diese Weise würden die Kompetenzen, die die Träger der Jugendsozialarbeit in der Vergangenheit bei der Ausbildung von sogenannten benachteiligten Jugendlichen erworben haben, eingebracht, ohne eine (weitere) Stigmatisierung zu riskieren, die mit der Ausbildung in Sondermaßnahmen immer auch verbunden sein konnte.

12 Lippegaus-Crünau, Petra: »Individualisierung in der betrieblichen Berufsausbildung. Entwicklungslinien und neue Ausbildungsmodelle«, in: Severing, Eckart/Weiß, Reinhold (Hg.): Individuelle Förderung in heterogenen Cruppen in der Berufsausbildung. Befunde - Konzepte - Forschungsbedarf, Bielefeld 2014, S. 21-38. 


\section{Den Blick auch auf den zweiten zentralen Lernort richten: Die Teilzeitberufsschule}

Tatsächlich bleibt die Teilzeitberufsschule, also der mit einem bestimmten Stundenanteil versehene zweite Lernort neben dem Ausbildungsbetrieb, unter dem Gesichtspunkt Migration/Integration weitgehend eine »black box«, während das Berufsschulsystem insgesamt als zentrale Institution im Übergang Schule - Arbeitswelt hohe Anteile von Schüler*innen mit Migrationsgeschichte aufweist.

Auch im eingangs referierten Sammelband zur Frage der Diskriminierung von Jugendlichen mit Migrationshintergrund in der Berufsausbildung wird die Berufsschule kaum thematisiert. Sie ist unter diesem Aspekt bisher kaum Gegenstand von Studien geworden. Dies ist umso verwunderlicher, als von betrieblicher Seite oftmals das Argument angeführt wird, dass die Scheiternsrisiken für Jugendliche mit Leistungsdefiziten eher in der Berufsschule und im »theoretischen« Schulstoff als im betrieblichen Teil der Ausbildung anzutreffen seien.

Einer grundsätzlichen Kritik wurde die Berufsschule zuletzt 2010 von Wolf-Dietrich Greinert und Stefan Wolfunterzogen. ${ }^{13}$ In historischer, pädagogischer und soziologischer Analyse zeigen sie, dass der eigenständige Bildungsauftrag der Teilzeitberufsschule durch ihre faktische Nachordnung im Verhältnis zur betrieblichen Ausbildung unterhöhlt wird. Trotz im Laufe der Jahrzehnte immer wieder vorgenommener Reformbemühungen, zu denen auch die Einführung der Oberstufenzentren in Berlin gehört, bleibt die Teilzeitberufsschule zum einen ein bildungspolitisches »Stief kind«, zum anderen, was wichtige Erkenntnisse über die dortigen Bildungs- und Lernprozesse angeht, wenig transparent.

Dies gilt insbesondere auch in Hinblick auf Auszubildende mit erheblichem Scheiternsrisiko und Jugendliche mit Migrationsgeschichte, ohne dies in irgendeiner Weise pauschal gleichsetzen zu wollen. Wo diese Aspekte überhaupt in den Blick geraten, geht es unter der Überschrift »Berufsschule« oder »Berufliches Bildungszentrum« oder »Oberstufenzentrum« meist um andere Bildungsgänge als jenen des »Dualen Systems«. Seit Kurzem erst äußern Kammern und Organisationen der Arbeitgeber Kritik am Zustand der

13 Greinert, Wolf-Dietrich/Wolf, Stefan: Die Berufsschule. Radikale Neuorientierung oder Abstieg zur Restschule? Frankfurt a.M. 2010. 
Berufsschulen. Im Januar 2012 meldet sich zunächst die Bundesvereinigung der Deutschen Arbeitgeberverbände (BDA) mit einer Art Memorandum zur Berufsschule der Zukunft zu Wort. ${ }^{14}$ Dieses Leitbild schlägt insofern neue Töne an, als es den eigenständigen und kooperativen Bildungsauftrag der Beruflichen Schulen betont. 2015 wird die Kritik explizit: Es fehle an moderne Ausstattung und an Qualitätssicherungen, die bundesweit gelten müssten, schließlich dürfe den Berufsschulen kein Geld entzogen, sondern es müsse im Gegenteil mehr investiert werden u.a. auch für »mehr individuelle Förderung ${ }^{15}{ }^{15}$ Auf der Basis einer IHK-Umfrage Ende 2015 wird für Berlin vor allem ein hoher Unterrichtsausfall hervorgehoben. ${ }^{16}$

\section{Der pädagogische Beitrag der Berufsschulen}

In diesen Kritiken wird auf strukturelle Probleme der Berufsschule abgehoben. Pädagogische Fragen im engeren Sinne sind wenig angesprochen. Sicherlich gibt es Zusammenhänge zwischen personeller und sachlicher Ausstattung und pädagogischer Qualität. Letztere ist jedoch auch ein eigenständiges Gestaltungsfeld, das in den Blick genommen werden muss.

In Hinblick auf die curriculare Orientierung an den Berufsschulen, die im gegebenen föderalen System Ländersache ist, hat es in den vergangenen Jahren wichtige Neuerungen gegeben, die sich stets an der Schnittstelle zwischen beruflich-theoretischer und allgemeiner Bildung bewegten. Insgesamt ist der Bildungsauftrag der Teilzeitberufsschule auf Handlungskompetenz ausgerichtet, die drei zentrale Dimensionen hat, nämlich Fachkompetenz, Personalkompetenz und Sozialkompetenz. Die pädagogische Annahme geht dahin, dass die erforderlichen Methoden- und Lernkompetenzen aus einer ausgewogenen Entwicklung dieser drei Dimensionen erwachsen.

\footnotetext{
14 BDA: Berufsschule der Zukunft, Berlin 2012.

15 Michler, Inga: Arbeitgeber bemängeln Qualität der Berufsschulen, in: Die Welt vom 22.2.2015

16 In demselben Artikel ist außerdem von einer von Bildungssenatorin Scheeres eingerichteten Arbeitsgruppe die Rede, die prüfen soll, wie die beruflichen Schulen gestärkt werden können. Köhler, Regina: In Berliner Berufsschulen fällt der Unterricht zu oft aus«, in: Berliner Morgenpost vom 16.11.2015.
} 
Folglich wären für die Berufsschule pädagogische Konzepte angemessen, die ganzheitlich und exemplarisch ausgerichtet sind und die auf einer reflexiven Beziehung auf die berufliche Praxis hin basieren. Hier setzt die Kritik an der oftmals vorfindlichen pädagogischen Praxis der Teilzeitberufsschulen an - ohne zu leugnen, dass es auch hier Schulen gibt, die pädagogisch besonders reflexiv und innovativ sind. So kommt z.B. Karin Büchter zu dem Schluss: Trotz landesrechtlicher und kultusministerieller Vorgaben und der Abhängigkeit vom regionalen Ausbildungsmarktgeschehen nutzen Berufsschulen schulbürokratische Freiräume und die Politikhaltigkeit der dualen Ausbildung dazu, um mit eigenen Profilen und besonderen Kompetenzen die duale Ausbildung in der Region mitzugestalten. ${ }^{17}$

Aber: Viele Berufsschulen folgen nach wie vor dem Schema der Abarbeitung von Stundentafeln, die nach Fachrichtungen organisiert und in Schulstundeneinheiten zerlegt sind. Die Bezüge zur beruflichen Praxis bleiben eher punktuell. Auch dort, wo in der Berufsschule selbst Werkstattunterricht stattfindet, ist dessen Verbindung zu den allgemeinbildenden Unterrichtsfächern nicht systematisch entfaltet. Die starke Dominanz des Fachberufs im Dualen System und die mangelnde didaktische Verknüpfung zwischen den fachlichen und allgemeinbildenden Unterrichtsinhalten führt oftmals zu einer Einschätzung geringerer Wertigkeit des allgemeinbildenden Unterrichts auch bei den Schülerinnen und Schülern.

Befragungen von Berufsschülern zeigen bei der Mehrheit eine stark positive Bewertung eines fachlich orientierten leistungsförderlichen Klimas. ${ }^{18}$ Aber immerhin ein Drittel der Befragten hat auch im dritten Ausbildungsjahr noch eine weniger strikt beruflich-betrieblich ausgerichtete Orientierung. Es liegt die Annahme nahe, dass es besonders die fachlich leistungsorientierten und gut betrieblich verankerten Berufsschülerinnen und Berufsschüler sind, die in der Teilzeitberufsschule, so, wie sie ist, ausreichend gute Lernbedingungen finden und von ihr profitieren. Aber was ist mit den anderen? In einem Berliner Workshop - organisiert von der WTK GmbH in Kooperation mit der Senatsverwaltung für Bildung, Wissenschaft und For-

17 Büchter, Karin: Berufsschulen in der dualen Ausbildung und regionalen Wirtschaft. Gleichberechtigte Partnerschaft durch Reformen (=Working Paper Forschungsförderung 059), Düsseldorf 2018.

18 Holl, Hans-Peter: »Gute< Lehrer an berufsbildenden Schulen aus der Schülerperspektive«, in: Die berufsbildende Schule 60/2008, S. $182 \mathrm{ff}$. 
schung - ging es um »Modelle und gute Beispiele für gelungene Integration aus beruflichen Schulen in Berlin«. Dort wurden als Bedingungen für eine gelingende Integration u.a. genannt: Individuelle Förderung, Transparenz des schuleigenen Unterstützungssystems, Bereitschaft des pädagogischen Personals, auch bei unterschiedlichen Qualifikationen im Team zu arbeiten, kollegiale Fallberatung, Sozialpädagog*innen als mögliche Schnittstelle für Kooperation, eine Kultur des Zuhörens mit Vertrauen in die Lösbarkeit von Konflikten und ein Miteinander statt Nebeneinander der Lernorte.

Diese wenigen Schlaglichter sollen darauf hinweisen, dass in integrationspolitischer Perspektive der Blick auch auf die Teilzeitberufsschule zu richten ist, und zwar insbesondere unter der Frage, mit welchen pädagogischen Konzepten sie die Ausbildung im Betrieb begleitet und reflektiert. ${ }^{19}$ Es kann nämlich sein, dass sich dort in besonderer Weise benachteiligende Verhältnisse kumulieren.

\section{Duales Lernprinzip: Betrieb und Berufsschule}

In einer pädagogischen Perspektive kann man über das »Duale Lernen« und die Lernorte Schule und Betrieb (und möglicherweise weitere außerschulische »Praxisorte«) so reden, als wären sie gleichgewichtige Teile. Im »Dualen System « der Berufsausbildung nimmt aber der Lernort Betrieb den Hauptanteil ein. Er dominiert das "System" nicht nur, was die zeitlichen Anteile betrifft, sondern vor allem auch aufgrund der Tatsache, dass das »Duale System « zwar rechtlich-öffentlich gefasst ist, aber zugleich privatwirtschaftlich funktioniert. Es sind die Betriebe, die darüber entscheiden, ob sie überhaupt, in welchen Berufen und in welchem Umfang ausbilden, und es sind auch die Betriebe, die darüber entscheiden, mit wem sie einen Ausbildungsvertrag abschließen. Ein Ausbildungsvertrag ist in diesem Sinne eine Sonderform eines Arbeitsvertrags.

19 Ob also z.B. die betriebliche Praxis, in der die Auszubildenden sich befinden, in exemplarischer Weise aufbereitet wird. Vergl. hierzu u.a. Kruse, Wilfried/Strauß, Jürgen/Braun, Frank/Müller, Matthias: Rahmenbedingungen der Weiterentwicklung des Dualen Systems beruflicher Bildung, Arbeitspapier 167 der Hans-Böckler-Stiftung, Düsseldorf 2009, S. 72-75. 
Die »Lernortfrage« ist in diese grundlegende Aufstellung der Berufsausbildung folgenreich eingeschlossen. Denn damit wird der Übergang von der Schule in die Berufsausbildung über einen Ausbildungsplatz-Markt vermittelt, mit Folgen in Hinblick auf die ungleiche Verteilung von Chancen auf einen Ausbildungsplatz. Da sich Ausbildungsplätze auch hinsichtlich ihrer späteren Verwertungschancen unterscheiden, gilt dies auch hinsichtlich der Zukunftsoptionen, die der jeweilige Ausbildungsplatz bietet. Diese systemische Problematik wird seit geraumer Zeit unter dem Aspekt der »Einzelbetriebsgebundenheit « der dualen Berufsausbildung diskutiert. ${ }^{20}$

Wenn der »Lernort Betrieb« also für den Aufbau einer stabilen beruflichen Perspektive jenseits der akademischen Welt (aber mit der Option auf späteren Zugang zu ihr) unverzichtbar ist, ${ }^{21}$ dann müssten sich gerade diejenigen Ausbildungsbetriebe, die eine besonders gute Ausbildung bieten, für Vielfalt öffnen. Betriebe sind also besonders gefordert.

\section{Pädagogische Lernortkooperation ist integrationspolitisch besonders wichtig}

Die beiden zentralen Lernorte Betrieb und Berufsschule müssen also für die Auszubildenden pädagogisch produktiv miteinander in Beziehung gebracht werden. Die Qualität dieser pädagogischen Lernortkooperation erweist sich als einer der Schlüsselfaktoren für eine erfolgreiche Öffnung von ungeförderter Berufsausbildung für Jugendliche und junge Erwachsene mit Migrationsgeschichte.

Eine Stärkung der Teilzeitberufsschule würde [...] die Unverzichtbarkeit des Betriebs im Kontext integrierter und übergreifender Lernprozesse demonstrieren. Auf diese Weise wird aus der Addition von Lernorten ein inhaltlich bestimmtes integriertes Lernkonzept: Weil der Betrieb als Lernort in der Beruflichen Bildung unverzichtbar ist, müssen Lernortstruktur und Cewichtung

20 Z.B. Kruse, Wilfried 2012: »Lernort Betrieb im >Dualen System«: Spannungsreiche Verhältnisse«, in: Kuda, Eva/Strauß, Jürgen/Spöttl, Georg/Kaßebaum, Bernd (Hg.): Akademisierung der Arbeitswelt? Zur Zukunft der beruflichen Bildung, Hamburg 2012, S. 158-169.

21 W. Kruse/J. Strauß/F. Braun/M. Müller: Rahmenbedingungen der Weiterentwicklung des Dualen Systems beruflicher Bildung, S. 72-75. 
unter bedeutsamer Aufwertung der Berufsschule - vor allem in qualitativer Hinsicht-neu geordnet werden. ${ }^{22}$

Eine Neubestimmung der Rolle der Teilzeitberufsschule im Berufsbildungssystem ist also überfällig und sie ist auch integrationspolitisch von erheblicher Bedeutung. Der Mehrwert "Dualen Lernens« bestünde dann gerade darin, dass sich das Lernen an den beiden Lernorten $\mathrm{zu}$ einem erneuerten gesellschaftlich gleichwertigen berufspraktisch basierten Bildungstyp zusammenfügt, ${ }^{23}$ in der die betriebliche Praxis einen erheblichen Schub an Neugier und Motivation auch für jenen Lernstoff bringt, um den es in der Berufsschule geht, und umgekehrt.

Das Wirksamwerden der kompensatorischen Funktion der beruflichen Bildung - und damit ihre mögliche Rolle beim Abbau von Bildungsbenachteiligung - ist kein Selbstläufer. Es ist von der Gestaltung der beruflichen Lernprozesse abhängig und hat umso mehr Chancen, je mehr er an in der Schulzeit geschaffenen Voraussetzungen anknüpfen kann. Vieles ist in den letzten Jahren im Feld der Berufsorientierung geschehen; hierfür gibt es gute Beispiele. Gerade vor diesem Hintergrund wird deutlich: Gute Berufsorientierung ist eine unverzichtbare Voraussetzung. Der Einstieg in Berufsausbildung und ihr erfolgreicher Abschluss für jene, die bisher »außen vor« geblieben sind, gelingen nur, wenn Betriebe und Berufsschulen sich (noch) weiter für Vielfalt oder Heterogenität öffnen. Zugleich müssen Betriebe und Berufsschulen hierbei das pädagogische Potenzial, das berufspraktisches Lernen und die duale Lernortskombination haben, (noch) wirksamer werden $\mathrm{zu}$ lassen. In diesem Sinne ist Ausbildungsqualität auch integrationspolitisch eine wichtige »Stellschraube .

22 Ebd., S. 74.

23 Ebd., S. 78. 


\section{Das Teilhabe-Potenzial der Berufsausbildung ausbauen und nutzen}

Ende 2016 führen der Berliner Beauftragte für Integration und Migration und die Arbeitsgemeinschaft Weinheimer Initiative ${ }^{24}$ gemeinsam in Berlin ein »JahresforumExtra« unter der Überschrift »Vielfalt in der Ausbildung» durch. In der Weinheimer Initiative arbeiten bundesweit Städte und Kreise zusammen, die sich für gute Übergänge von der Schule in die Arbeitswelt und gelingende Bildungsbiografien engagieren. Mit dem Ansatz des Berliner Integrationsbeauftragten gibt es also erhebliche Schnittmengen. In der »Gemeinsamen Erklärung« aus Anlass des Jahresforums heißt es u.a.:

»Auch Jugendliche, die motiviert und gut »beruflich orientiert" sind, scheitern an Auswahlentscheidungen der Betriebe. Die Ablehnungen haben vielfach ihren Grund in der Erwartung, dass diese Jugendlichen, die in der Ausbildung und in den Abschlussprüfungen geforderten Leistungen nicht bewältigen (könnten). Tatsächlich ist die Zahl der Ausbildungsabbrüche nach Ausbildungsbereichen und Gründen sehr unterschiedlich - hoch.

Das ist ein erstaunlicher Tatbestand: Das enorme Bildungs- und Sozialisations-Potenzial berufspraktischen Lernens, also das Lernen nicht unter schulischen, sondern unter sogenannten Ernstbedingungen, gehört zu den festen Überzeugungssätzen vor allem auch der Vertreter des >Dualen Systems $<$. De facto scheint es aber erhebliche und für die Jugendlichen folgenreiche Zweifel an der pädagogischen Leistungsfähigkeit dualer Berufsausbildung zu geben. Die Ausgrenzungspraxis kritisiert heftig manche Postulate aus Sonntagsreden. ${ }^{25}$

Es ist eben nicht selbstverständlich, dass Jugendliche, die ohne einen positiven Bezug zur Arbeitswelt aufwachsen, Interesse an einem Beruf gewinnen, sich kümmern und sich bewerben. Dann aber, wenn sich dieses Interesse entwickelt hat, schließlich doch keinen Ausbildungsplatz zu erhalten, untergräbt die Motivation. Das Gefühl, nicht dazu zu gehören, bestätigt sich erneut. Erneutes Scheitern trotz Motivation und Anstrengung breitet sich als Negativbotschaft aus: in den Familien, in den Schulklassen und den Freundeskreisen. Das sind verheerende Effekte.

\footnotetext{
24 www.kommunale-koordinierung.de

25 www.kommunale-koordinierung.de/uploads/tx_news/JFX_2016_Berlin_Positionierung_01112endg.pdf vom 14.12.2016.
} 
Öffnung der Ausbildung für Vielfalt im umfassenden Sinne ist ein Prüfstein für die Teilhabe-Chancen in der Einwanderungsgesellschaft. Die tatsächliche Öffnung der Berufsausbildung für jene, die bisher abseits gestanden haben, gehört gegenwärtig zu den zentralen Herausforderungen. Erfolgreiche Berufsausbildung und gelingender Einstieg in eine Fachbeschäftigung können die Basis für eine selbständige Lebensführung ohne dauerhafte staatliche Transferleistungen sein. Ausbildungslosigkeit droht eine große soziale Minderheit zu verstärken, die in vielfacher Hinsicht von gesellschaftlicher Teilhabe ausgeschlossen sind. Damit verstärken sich Tendenzen zur sozialen Spaltung. ${ }^{26}$ Soziale Spaltung aber bedroht den Zusammenhalt und die demokratische Kultur unserer Gesellschaft.

\section{Vielfalt in der Ausbildung}

Aus den Beiträgen, Debatten und der vorgelegten Erklärung des gemeinsamen Jahresforum $\mathrm{s}^{27}$ wurden die folgenden Handlungsbedarfe deutlich:

1. Das pädagogische Potenzial praktischen Lernens in Betrieben und Berufsschulen muss ausgebaut und verstärkt genutzt werden. Dies gilt insbesondere auch für die dringende Vertiefung der pädagogischen Lernortkooperation, mit dem Ziel, auch Jugendliche mit schlechteren Startbedingungen einen erfolgreichen Ausbildungsabschluss zu ermöglichen. Begleitende Maßnahmen zur Ausbildungssicherung müssen hinsichtlich ihrer Wirksamkeit justiert und vor allem in einen tragfähigen kooperativen Zusammenhang gebracht werden.

2. Der Zugang zur Berufsausbildung muss für Jugendliche, die bislang im Zuge der überkommenen betrieblichen Auswahlverfahren gescheitert wären, erleichtert werden. Dies kann insbesondere durch eine stärkere positive Bewertung erfolgreich absolvierter Betriebspraktika, die in gute

$26 \mathrm{Vgl}$. hierzu U. Bochum/J. Butler/K. Kohlmeyer/S. Odenwald: Soziale Spaltungen in Berlin.

27 Eine Dokumentation der Beiträge findet sich unter: www.kommunale-koordinierung.de/ aktivitaeten/jahresforen/jahresforum-extra-2016-berlin/; Vgl. auch Der Beauftragte des Berliner Senats für Integration und Migration ( $\mathrm{Hg}$.): Alle mit dabei? Potenziale der Berufsausbildung für Jugendliche aus Einwandererfamilien, Berlin, Januar 2019. 
schulische Berufsorientierungsprozesse eingebettet sind, erreicht werden (Stichwort: Qualifizierte Vierstufigkeit).

3. Eine transparente, auf die Entwicklung der Jugendlichen orientierte einheitliche Dokumentation der Betriebspraktika ist hierfür Voraussetzung.

4. Berufsorientierung ist als ein kooperativer Prozess (Stichwort: Lernallianzen) zu verstehen: Kooperationsmodelle zwischen allgemeinbildender Schule und Berufsschule und zwischen allgemeinbildender Schule und Betrieben müssen ausgebaut, gesichert und mit förderlichen Rahmenbedingungen versehen werden.

5. Insgesamt muss den berufsbildenden Schulen als zentrale Institution im System des Übergangs und der Berufsausbildung verstärkt bildungspolitische Aufmerksamkeit gewidmet werden. Sie müssen - auch in enger Kooperation untereinander - zu lokalen berufsbildenden Kompetenzzentren weiterentwickelt werden.

6. Die Berufsorientierung an sogenannten $» B r e n n p u n k t s c h u l e n «$ muss verstärkt werden. Hierzu sind nicht nur Kooperationsmodelle auszubauen und zu sichern, sondern die Verankerung der Berufsorientierung als "Aufgabe der ganzen Schule« muss in der Lehrerschaft stärker gefestigt und personell gestärkt werden.

7. Wirkungsvolle Verstärkung von Berufsorientierung und Öffnung von Ausbildung für Jugendliche, die bisher »abseits gestanden« haben, braucht auch begleitendes Empowerment für diese Jugendlichen selbst: Stadtteilarbeit und »Clubs« gehören zu den Ansätzen, die Erfolg versprechen. Sie müssen stärker als bisher kooperativ verknüpft werden.

8. Die institutionellen Ansätze zur Förderung des Übergangs von der Schule in die Arbeitswelt, wie z.B. kommunale Koordinierung, bzw. die entsprechenden Landesprogramme, müssen um "Erfolgreiche Ausbildung « erweitert werden. Die Transferagenturen sind aufgefordert, dies zu einem ihrer Orientierungspunkte bei der Beratung vor Ort zu machen.

9. In den lokal-regionalen "Übergangssystemen" müssen Arbeitsteilung und Kooperation um die Akteure der Berufsausbildung bzw. der Arbeitswelt erweitert werden, also um die beruflichen Schulen, die Berufsbildungsausschüsse der Kammern, wichtige "Leitbetriebe«, um Jugendberufsagenturen und die Agenturen für Arbeit. Für alle diese Handlungsfelder gibt es bereits erprobte und aussichtsreiche Praxis, die als Modell genutzt werden kann. 
10. Von daher ist ein wichtiges Erfordernis, unter der Prämisse von »Vielfalt in der Ausbildung " gute Praxis zu dokumentieren, erfahrbar und »begehbar« zu machen und Schritte einzuleiten, erprobte und belastbare "gute Praxis« in Regelbausteine zu überführen.

\section{Fazit}

Berufsausbildung kann eine gute Option für junge Menschen sein, die mit der tradierten Form des schulischen Lernens nicht gut zurechtgekommen sind oder auch sonst auf ihrem Bildungsweg mit verschiedenen Schwierigkeiten zu tun haben, weil Lernen in der betrieblichen Praxis unter bestimmten Bedingungen einen guten Raum für Motivation und den raschen Aufbau von Fähigkeiten bereitstellt. Ein solches Potenzial hat Lernen in der betrieblichen Praxis allerdings nur dann, wenn es pädagogisch-didaktisch von hoher und den Lernenden angemessener Qualität ist. Dies ist eine Prämisse, die - neben dem Ziel der gleichberechtigten Teilhabe - den Ansatz des integrationspolitischen Leitprojekts Berlin braucht dich! mitbegründete. In diesem Kapitel wurde - gewissermaßen spiegelbildlich zu Kapitel 6 - der Begründung für diese Prämisse nachgegangen, wie sie sich aus Studien und Erfahrungsberichten ergibt.

Diese Prämisse müsste also auch für die über Betriebsbegegnungen gut motivierten Bewerber*innen aus den im Konsortium mitarbeitenden Schulen gelten und sich für sie also ein Einstieg in Ausbildung öffnen. In Kapitel 5 wurde gezeigt, dass dies selten der Fall ist, in Kapitel 6 wurden die Umstände, unter denen viele dieser jungen Menschen, die aus Familien mit Einwanderungsgeschichte kommen, ihren Weg zu finden versuchen, beschrieben. Neben den Erfahrungen aus Berlin braucht dich! zeigen auch verschiedene Studien, dass ein Scheitern in den betrieblichen Auswahlverfahren häufig ist.

Die Studien belegen, dass bei Bewerbungen und Auswahl angewendete Instrumente, Verfahren und Kriterien in vielen Fällen jene benachteiligen, die nicht in das vorgefasste betriebliche Bild eines oder einer Auszubildenden passen. Während eine migrantische Herkunft für sich genommen immer weniger zu einem Einstellungsvorbehalt führt, ist es die Befürchtung, dass die Auszubildende oder der Auszubildende in der Ausbildung scheitern oder sie nur mit einem erheblichen zusätzlichen betrieblichen Aufwand an 
Unterstützung geschafft wird, die zu Ablehnung führt. Es verbinden sich also Einschätzungen von Risiken, die aus vermeintlicher ethnischer Differenz entstehen könnten, mit solchen, die aus der Erwartung von Mängeln in Lern- und Leistungsfähigkeit resultieren könnten.

Es spricht also einiges dafür, dass Betriebe dem kompensatorischen Potenzial, das Lernen im Medium von Beruf und Betrieb darstellt, nicht wirklich trauen, also an ihrer Fähigkeit als Ausbildungsbetrieb zweifeln, ohne erhebliche Zusatzinvestitionen Auszubildende mit einer erwarteten Leistungsschwäche erfolgreich zum Abschluss zu bringen, obwohl es viele Beispiele von jungen Erwachsenen gibt, die in der Ausbildung geradezu einen Leistungssprung machen.

Beim Durchgang durch die möglichen Gründe kommen insbesondere zwei Aspekte in den Blick, nämlich die Frage, ob die pädagogisch-didaktische Gestaltung der Ausbildung auf Vielfalt, also auf notwendige Binnendifferenzierung und Individualisierung von Lehren und Lernen, angelegt ist, und wie es mit der pädagogischen Kooperation zwischen Betrieb und dem zweiten Lernort Berufsschule bestellt ist. In beiderlei Hinsicht scheint es Mängel zu geben, wobei wohl insbesondere der pädagogischen Lernortkooperation eine bisher im Leitprojekt vernachlässigte integrationspolitische Bedeutung zukommt.

Ausbildungsqualität wird so zu einer Schlüsselfrage, und dies wiederum in zweierlei Hinsicht: zum einen, um auch bei startschwächeren Auszubildenden eine begründete Erwartung hinsichtlich ihres Ausbildungserfolgs zu haben, zum anderen - eng damit verbunden -, um sich als Ausbildungsbetrieb darauf einlassen zu können, die bisherigen Auswahlverfahren so zu modifizieren, dass junge Menschen, die sich entschieden haben und für eine Ausbildung motiviert sind, überhaupt in sie eintreten können.

Welches Potenzial das Lernen im Medium von Beruf und Betrieb hat, und wie eine den heuten Verhältnissen angemessene Ausbildungsqualität aussehen müsste, war Thema einer Konferenz zu »Vielfalt in der Ausbildung«, auf die in diesem Kapitel ebenfalls Bezug genommen wurde. Sie zeigte, dass in niemand der Beteiligten »bei Null anfangen « muss.

Dass junge Menschen, die bisher kaum eine Chance auf einen Ausbildungsplatz hatten, ihre Interessen und Motivationen aber im Rahmen auf Betriebsbegegnungen aufbauenden Berufsorientierung geklärt haben und motiviert sind, tatsächlich einen Ausbildungsvertrag erhalten und Aussicht auf eine erfolgreiche Berufsausbildung haben, ist integrationspolitisch - 
und damit eng verbunden: für den sozialen Zusammenhalt der Stadt - fundamental. Denn ein Scheitern auf breiter Front kann - um an die Einsichten aus dem vorherigen Kapitel zu erinnern - zu einem Motivationsverlust weit über den Kreis der unmittelbar Betroffenen hinausführen und das weit verbreitete Gefühl, zu den »Verlierern« zu gehören, noch verstärken.

Hieraus folgen eine Reihe von Handlungserfordernissen, mit denen das Kapitel abschließt. Die Kapitel 6 und 7 liefern - miteinander korrespondierende - Einsichten und Argumente, die einer Re-Orientierung des integrationspolitischen Ansatzes zur Öffnung von Berufsausbildung helfen können; eine Re-Orientierung, die in gewisser Weise auch eine erfahrungsbasierte Rückkehr zu den Startzielen des Jahres 2007 ist. Das folgende Kapitel diskutiert dies. 



\section{Kapitel 8}

\section{Ausbildung für Vielfalt öffnen: Ein neuer Anlauf}

Mitte 2015 beginnt aktiv die Re-Orientierung von Berlin braucht dich!, die in gewisser Weise ein »back to the roots« bedeutet. Denn es wird wieder über die Öffnung der Ausbildung gesprochen und die Betriebe werden - nach Jahren, in denen es vor allem um Berufsorientierung ging - zum primären Adressaten.

Im Unterschied zum Start kurz vor der Jahrzehntwende geht es nun aber nicht mehr ganz allgemein um die Öffnung für Jugendliche mit Migrationsgeschichte - dieses integrationspolitische Anliegen war mittlerweile bei den Betrieben gut angekommen -, sondern um die Öffnung für jene Jugendlichen mit Migrationsgeschichte aus den im Konsortium mitarbeitenden Schulen, die sich für eine Ausbildung interessieren, in Praktika ihre Motivation und ihre Aufnahmefähigkeit gezeigt haben, und dennoch bislang bei der Ausbildungsplatzvergabe leer ausgingen.

Nach mehreren Jahren der konsortialen Zusammenarbeit von Schulen und Betrieben in Berlin braucht dich! mussten die geringen Übergänge in Ausbildung aus den beteiligten Schulen als ein problematisches Signal verstanden werden, sowohl bei den Schüler*innen und in deren Lebensumfeld, als auch bei den Lehrer*innen und Schulleiter*innen, die sich engagierten. Über solche naheliegenden negativen Effekte war auch schon in verschiedenen Arbeitskreisen von der Schulseite aus berichtet worden. 


\section{Konsortialtagung 2015: Den Betrieb als Lernort mit Potenzial wiederentdecken}

Auf der Konsortialtagung am 12. Juni 2015 wird im Hauptreferat mit dem Titel »Wiederentdeckt: Der Betrieb als Lernort mit Potenzial« Ausbildung und ihre Öffnung deshalb direkt zum Thema. ${ }^{1}$ Erinnert wird daran, warum Betriebsbegegnungen Motivationsschübe für Ausbildung auslösen können: Die praktischen Anforderungen im Betrieb wecken die praktischen Talente, Betriebe sind Orte für Erwachsene, die »Ernstsituation« im Betrieb verlangt von den Jugendlichen Verbindlichkeit. Und: Es kommt in der Zusammenarbeit mit anderen präzise darauf an, Aufgaben gut und in der hierfür vorgesehenen Zeit zu erledigen. Schüler*innen vollziehen so den Schritt in eine andere Welt, in der Anforderungen und Normen in erster Linie vermittelbare und nachvollziehbare sachliche Gründe haben und keine abstrakten Lernstoffe sind.

In der langjährigen Praxis von Berlin braucht dich! sei diese erhoffte Wirkung zwar eingetreten und das erwachte Interesse an einer Berufsausbildung sei auch in Bewerbungen gemündet. Aber, resümiert das Referat im Gleichklang zur in den vorherigen Kapiteln entwickelten Bewertung, zu wenige dieser Jugendlichen erhielten dann tatsächlich einen Ausbildungsvertrag, wahrscheinlich deswegen, weil Betriebe offenbar befürchteten, dass sie diese Jugendlichen nicht - oder nur mit großer Mühe - zu einem erfolgreichen Ausbildungsabschluss bringen können. Dies aber unterschätze das enorme Bildungs- und Anregungspotenzial des Lernorts Betrieb für fachliche und persönliche Bildung, dessen Nutzung dazu führe, dass auch jene, denen man dies zunächst nicht zutrauen würde, ohne Absenkung der fachlichen Anforderungen zu einem erfolgreichen Abschluss kommen können.

Damit wurde ein Impuls gesetzt, die bisherigen Einstellungsverfahren und deren Annahmen auf den Prüfstand zu stellen. In ihrem Beitrag gab dann Kerstin Oster, Personalvorstand der Berliner Wasserbetriebe, schon auf dieser Tagung eine Richtung vor, der Berlin braucht dich! in den folgenden Monaten folgen sollte: »Wir müssen uns fragen: Sind die Auswahlkriterien überhaupt noch die richtigen? Wir müssen die Möglichkeit schaffen,

1 Kruse, Wilfried: »Wiederentdeckt. Der Betrieb als Lernort mit Potenzial«, https://www.berlin-braucht dich.de/fileadmin/user_upload/PDFs/Wiederentdeckt_Lernort_Betrieb.pdf 
uns auch von den Noten lösen zu können und uns daran orientieren, wer das größte Interesse und Potenzial hat. $\aleph^{2}$

\section{Die Auswahlkriterien für Ausbildungsplätze werden hinterfragt}

Die Konsortialtagung 2015 konnte also durchaus als eine Aufforderung verstanden werden, einen Ansatz zu entwickeln, der Ausbildung für Jugendliche mit Migrationsgeschichte weiter öffnet. Es sollte noch über ein Jahr dauern - nämlich bis zum Beginn des Ausbildungsjahres 2016 -, bis daraus erste Praxisansätze entstanden. Zunächst musste der Übergang in Ausbildung als Gestaltungsfeld etabliert werden, ohne die bisherige Arbeit auszusetzen. Dabei konzentrierten sich Überlegungen auf eine Kritik an den bisherigen und nach wie vor praktizierten Auswahl-und Einstellungsverfahren.

Es wurde davon ausgegangen, dass diese Verfahren - so unterschiedlich im Einzelnen ihre Elemente, wie Bewerbungsschreiben, Tests, praktische Übungen, Auswahlgespräche gewichtet wurden - in der Regel zu einem Ausschluss von Jugendlichen führen, die nicht dem »Modell« der schulisch durchschnittlich guten und verhaltenssicheren jungen Männer und Frauen entsprechen, wie sie vorzugsweise in der Vergangenheit in Ausbildung genommen wurden.

Insbesondere wurde davon ausgegangen - und in späteren Analysen auch bestätigt -, dass diese Einstellungsprozeduren insofern auch diskriminierende Effekte gegenüber bestimmten Gruppen von Jugendlichen mit Migrationsgeschichte zeigen, als sie die Beherrschung der deutschen Sprache sehr hoch bewerten, und zwar unabhängig davon, ob dies im Ausbildungsberuf in dieser Weise angezeigt ist. In Auswahlgesprächen werden, so die Annahme, bestimmte Codes, also Sprachformeln, erwartet, die die jungen Leute aufgrund der Betriebsferne ihrer Milieus nicht gelernt haben. Zugleich wird auf Verhaltensebene wenig Toleranz gegenüber Verunsicherungen und dem empfindlichen Selbstbewusstsein aufgebracht, die aber für Jugendliche aus eher sozial belasteten Milieus durchaus normal sind.

Diese Kritik war nicht neu, wurde aber vom beauftragten Träger BQN noch einmal aufgearbeitet und präzisiert und auf konkretes Anschauungs-

2 Dokumentation Konsortialtagung BQN 2015, S. 9, www.bqn-berlin.de > site > doku_konsortialtreffen_2015. 
material aus den beteiligten Betrieben bezogen. ${ }^{3}$ Nun sind aus Sicht der Betriebe Einstellungsprozeduren, so problematisch sie im Einzelnen auch sein mögen, eine sinnvolle Operation, denn sie versprechen, junge Leute als Auszubildende in den Betrieb zu nehmen, die mit hoher Wahrscheinlichkeit in der Lage sind, die Anforderungen der Ausbildung in Berufsschule und Betrieb jedenfalls ohne große zusätzliche Unterstützungsleistungen durch die Betriebe zu bewältigen. »Einstellungsprozeduren« sollen also aus betrieblicher Sicht im Rahmen des Möglichen Ausbildungsabbrüche, hohe Zusatzkosten zu den ohnehin kostspieligen Ausbildungen und Fehlinvestitionen vermeiden helfen. Diese »Ratio« wurde zu Beginn der Neu-Orientierung wenig ins Kalkül gezogen u.a. deshalb, weil zunächst eine Diskriminierungskritik im Zentrum der Neu-Orientierung stand.

\section{Differenzierte Sicht auf die Schüler*innen, um die es geht}

Die erneute Aufmerksamkeit auf die tatsächlichen Eintritte aus der Schule in die Ausbildung unter Berücksichtigung des Einstellungsvorhalts der Ausbildungsbetriebe setzte (erneut) die Frage auf die Tagesordnung, welche Schüler*innen aus der Gesamtheit eines Jahrgangs das hauptsächliche Potenzial für den Weg in eine Berufsausbildung ausmachen. Vor dem Hintergrund der Erfahrungen, die in Berlin braucht dich! über die Jahre gesammelt wurden, lag folgende Differenzierung innerhalb der Schülerschaften der höheren Klassen der Sekundarschulen nahe, die Klaus Kohlmeyer, Projektleiter von BQN, vorschlug:: eine kleinere Gruppe, die Ambitionen und Voraussetzungen für einen weiteren Schulweg in Richtung Abitur hat, eine weitere) Gruppe, die von ihren Voraussetzungen her zunächst nicht für einen direkten Einstieg in eine Ausbildung infrage zu kommen scheint und die größte, mittlere Gruppe mit einer potenziell hohen Bereitschaft und Fähigkeit für eine Ausbildung.

Um die Notwendigkeit einer gezielten Aufmerksamkeit dieser größten Gruppe gegenüber zu unterstreichen, wurde argumentiert, dies sei ein Potenzial für qualifizierte Fachleute, das zu ignorieren sich die Stadt, denen der Nachwuchs für diese Bereiche ausgehe, und die beteiligten Betriebe

3 Neuhof, Ursula/Yazar, Serdar/Gębala, Marta: Werkseinstellungen auf Vielfalt setzen (=Impulse zu Vielfalt 2018/2), Berlin 2018. 
nicht leisten könnten. Diese Figur eines gravierenden künftigen Fachkräftemangels spielte nun im Vergleich zur Startphase von Berlin braucht dich! eine viel größere Rolle.

Vor dem Hintergrund ein solchen vorgeschlagenen Differenzierung wurde ein sehr positives Bild der starken Voraussetzungen gezeichnet, die diese Mittelgruppe von Schüler*innen für eine Ausbildung mitbringt (»Sie können Ausbildung «) und gewissermaßen auch verteidigt. Belegt wurde dies insbesondere durch die Erfahrungen mit verschiedenen dieser jungen Leute im Zuge von Betriebsbegegnungen und - vereinzelt - als Auszubildende.

\section{Grundzüge eines "Pilotvorhabens" zu neuen Wegen in die Ausbildung entstehen}

Auf diese Weise avancierte das "Erfolgreiche Absolvieren der Vierstufigkeit" zum Angebot eines Äquivalents für die üblichen Einstellungsprozeduren. Die Praktika sollten als Erprobungsfeld betrachtet und als Basis für eine Entscheidung über den Eintritt in die Ausbildung genommen werden.

Damit wurde eine sinnvolle Rückbindung an die gemeinsamen Anstrengungen der letzten Jahre im Konsortium zur Etablierung der Vierstufigkeit vorgenommen. Zugleich aber schleppt die unterstellte "Schlüsselfunktion" der - immer noch häufig punktuellen - Betriebsbegegnungen ihre aus der Anfangsphase von Berlin braucht dich! stammende Überbewertung für den Aufbau stabiler Berufsorientierungen und offener und mutiger Lernhaltungen mit. Allerdings geschah dies skizziert nicht ungebrochen: Zum einen wurde eine Einbettung der Betriebsbegegnungen in eine systematische schulische Berufsorientierung (als Querschnittsaufgabe der "ganzen Schule«) in der praktischen Arbeit mit den Schulen zum zentralen Thema gemacht, zum anderen entstand die Idee der Berlin-braucht-dich!-Clubs zur Stärkung derjenigen Jugendlichen, die sich wirklich und willentlich auf den Weg machen.

Erwartet war von vornherein, dass nicht alle in Berlin braucht dich! mitwirkenden Betriebe aus dem Stand bereit sein würden, eine gewisse Zahl von Ausbildungsplätzen für junge Leute mit diesem Hintergrund zu reservieren. So entstand die Rede vom »Piloten«, die rasch zu einer Art Label für die neue Phase wurde.

Es wurden Gespräche mit der Leitungsebene der mitarbeitenden Betriebe mit Landesbeteiligung aufgenommen, die z.T. auch unter Beteiligung des Integ- 
rationsbeauftragten, immer aber in seinem Auftrag vom Geschäftsführer von BQN geführt wurden. Ziel war, zu Vereinbarungen mit den Betrieben über ihre Beteiligung am »Piloten« zu gelangen. Die Konzentration auf die Betriebe mit Landesbeteiligung erfolgte, weil sie langjährige Partner in Berlin braucht dich! und als sehr gute Ausbildungsbetriebe bekannt sind und als Betriebe des öffentlichen Sektors eine herausgehobene soziale Verantwortung haben.

Tatsächlich konnten eine Reihe von Vereinbarungen abgeschlossen werden, in denen die Betriebe jeweils ein Kontingent an Ausbildungsplätzen bereitstellten, ohne dass die Bewerber*innen die übliche Einstellungsprozedur - mit ihrem Kern: den Tests - absolvieren mussten, bzw. ohne dass die Ergebnisse solcher Einstellungsprozeduren, falls sie dennoch durchgeführt würden, als Ablehnungsgründe gebraucht werden konnten. Neben formalen (Alter) und gesundheitlichen Aspekten kamen insofern bei der Besetzung dieser "reservierten" Ausbildungsplätze zwei weitere Kriterien zum Zuge: die »Bewährung« im Praktikum und die weitere Betreuung im Rahmen von »Clubs«. De facto spielten auf diese Weise die Empfehlungen durch das BQN-Team eine erhebliche Rolle.

\section{Schulen und Betriebe wechselseitig aufeinander angewiesen}

Bei der Besetzung der bereitgestellten Ausbildungsplätze trat allerdings eine weitere Ernüchterung ein: Es fanden sich in den beteiligten Schulen nicht genug junge Leute, die sich für eine Ausbildung in diesen Betrieben und für die angebotenen Berufe interessierten und einen erfolgreichen Durchgang durch die »Vierstufigkeit« aufweisen konnten. Schließlich und endlich wurden neben den persönlichen Ambitionen der Jugendlichen die Erfahrungen aus den dreiwöchigen Betriebspraktika (in Klassenstufe 9) - teilweise auch außerhalb von Berlin braucht dich! - und aus ergänzenden Praktika in Jahrgangsstufe $10 \mathrm{zu}$ Rate gezogen.

Es zeigte sich hier also sehr deutlich, dass es in den beteiligten Schulen bisher nicht oder nur schwach gelungen war, im Feld von Berufsorientierung die individuelle Entwicklung der einzelnen Schüler*innen im Blick zu behalten oder in den Blick nehmen. Dies zwang dazu, die Schulen und ihren Beitrag zum Aufbau von beruflichen Perspektiven erneut zu befragen; ohne dies in eine einseitige Schulkritikmünden zu lassen. 
Allmählich wuchs bei allen Beteiligten ein Verständnis davon, dass der Erfolg des »Piloten « nicht nur davon abhängig ist, ob die Aktivitäten von Betrieben und Schulen miteinander korrespondieren, sondern auch, ob sie in einem wechselseitigen Verhältnis der Schaffung günstiger Voraussetzungen aufeinander bezogen sind.

Was ist damit gemeint? Es geht um das Aufrechthalten einer positiven Dynamik, die dadurch ausgelöst wurde, dass eine Gruppe von Betrieben zunächst mit dem Verzicht auf die bisher üblichen Einstellungsverfahren und -Tests ein sehr wichtiges Signal gesetzt hatte. Dies musste von Schulen aufgegriffen und "weiterverarbeitet« werden, um immer bessere Voraussetzungen dafür zu schaffen, dass (1) die in Ausbildung einmündenden Jugendlichen hierfür besser vorbereitet sind und (2) die Zahl derjenigen, die nach der Schule in eine Ausbildung einmünden, von Jahr zu Jahr steigt. Die Betriebe, die ihre Beteiligung am »Piloten« als ein Experiment ansehen, müssen (1) schon aus Eigennutz Vorkehr treffen, dass die Jugendlichen nicht in der Ausbildung scheitern, was vor allem eine pädagogische Herausforderung der Gestaltung des Ausbildungsgeschehens und der Zusammenarbeit mit den Teilzeitberufsschulen bedeutet, und (2) ebenfalls Schritt für Schritt die Zahlen erhöhen, auch dadurch, dass mehr Betriebe gewonnen werden, sich am »Piloten« $z u$ beteiligen. Dies kann als Impuls auf die Schulen und die Schülerschaft zurückwirken, die sehen, dass es die Anstrengungen lohnt, was erneut die Betriebe stimuliert usw.

Senatorin Breitenbach zielte in einer Rede auf dem Konsortialtreffen im Juni 2017 auf eine solche, aus einer engen Zusammenarbeit resultierenden Vorwärtsbewegung, wenn sie von dem notwendigen Durchbruch sprach, der nun erreicht werden müsse, und dessen Erfolgskriterium der erfolgreiche Ausbildungsabschluss jener Jugendlichen sei, denen man dies bisher nicht zugetraut habe. ${ }^{4}$

\section{Das »Setting " von Berlin braucht dich! muss angepasst werden}

Nach der Vereinbarung mit den Betrieben über die Teilnahme am »Piloten« musste dieser aber zunächst einmal in die komplexe »Architektur « von Berlin braucht dich! mit seiner umfangreichen Partnerschaft von über 60 Betrieben

4 www.bqn-berlin.de > files > bqn_dokumentation_160718 
und Verwaltungen, 25 Schulen und weiteren Kooperationspartner*innen, mit dem breiten Spektrum von Tätigkeiten, die durch das Team von BQN durchgeführt werden, und mit seinen Beratungs- und Steuerungsstrukturen eingearbeitet werden. ${ }^{5}$

Das Pilotvorhaben erzwingt aufgrund seiner Bedingungen, wie sie kurz skizziert wurden, eine Schwerpunktsetzung innerhalb des Aktivitätsspektrums und des - immer begrenzten - Ressourceneinsatzes, z.B. eine erhöhte Aufmerksamkeit gegenüber den Betrieben, die sich am Pilotvorhaben beteiligen, eine gezielte Begleitung der jungen Leute, die für die reservierten Ausbildungsplätze infrage kommen, einschließlich der Entwicklung und Erprobung neuer Formate, wie der Berlin braucht dich!-Clubs. Aber was ist mit den anderen Betrieben, die sich nicht oder noch nicht am Pilotvorhaben beteiligen? Werden sie gewissermaßen zur 2. Liga innerhalb des Konsortiums? Und was ist mit den Schulen? Zwischen den Schulen gibt es Unterschiede in der Art und Weise, wie sie mit Berufsorientierung umgehen und wie stark die sie Impulse von Berlin braucht dich! Aufnehmen. Wäre es nun ein richtiger Schritt, parallel zu den »Pilotbetrieben« auch eine Gruppe von »Pilotschulen« zu bilden? Und wäre dies dann so zu verstehen, dass die hauptsächliche »Zufuhr« von jungen Leuten zu den »Pilotbetrieben« auch von dort käme? Man braucht die Fragen nur zu formulieren, um ihre Brisanz zu erkennen. Denn das, was das Konsortium insgesamt leistet, nämlich sich an der Qualifizierten Vierstufigkeit gemeinsam abzuarbeiten, ist wegen der Teilnahme gerade dieser Schulen und dieser Betriebe integrationspolitisch sehr wertvoll und darf nicht gefährdet, sondern muss möglichst pragmatisch auf Kontinuität gestellt werden.

Versteht man das Pilotvorhaben nicht als vom Konsortium sich lösender eigener "Verein«, sondern als einen Motor der Weiterentwicklung auch innerhalb und für das Konsortium selbst, dann wird der interne Transfer (neben dem Transfer nach »außen«, also zu Betrieben, Schulen und der fachlichen Öffentlichkeit außerhalb des Konsortiums) $z u$ einer wichtigen und anspruchsvollen Aufgabe. Hier nimmt der klassische Öffentliche Dienst, insbesondere was die Senatsverwaltungen und die Bezirksämter betrifft, eine Sonderstellung ein, weil dieser sich trotz formaler Zugehörigkeit zu Berlin

5 Die 2016 erschienene Broschüre mit dem Titel »Zukunft ist keine Frage der Herkunft« gibt hierzu einen guten Überblick. https://www.berlin-braucht-dich.de/fileadmin/user_upload/PDFs/BQN_BR_Image_A5_7.VL.pdf 
braucht dich! an der Vierstufigkeit bisher nur unterdurchschnittlich bis nahezu überhaupt nicht beteiligt hat. Gleichzeitig klafft aber im Öffentlichen Dienst aufgrund des demographischen Wandels und der wachsenden öffentlichen Aufgaben in den nächsten Jahren eine eklatant große Nachwuchslücke.

\section{Ausbildung: eine Frage der Einstellung? Erstmal erproben ...}

In der Folge der Vereinbarungen, die mit einer Anzahl von Betrieben zum Pilotvorhaben »Erprobung neuer Zugänge in die Ausbildung « abgeschlossen wurden, und parallel bzw. nach der Einarbeitung dieses Vorhabens in die "Architektur« von Berlin braucht dich! stehen nach der Konsortialtagung 2017 tatsächlich Betriebe bzw. Arbeitgeber erneut im Zentrum der Aufmerksamkeit. Es handelt sich also tatsächlich um eine Art. »back to the roots", wenn auch vor dem Hintergrund veränderter Rahmenbedingungen und vor allem der in den vergangenen Jahren gesammelten gemeinsamen Erfahrungen.

Zur Unterstützung des neuen Anlaufs, Ausbildung für Vielfalt zu öffnen, entwickelte der Träger BQN im Auftrag des Integrationsbeauftragten und in enger Abstimmung mit Betrieben erneut eine bemerkenswerte PlakatKampagne. Fotos und Firmenlogos signalisieren die aktive Rolle der Betriebe und schließen insofern an die Startkampagne von Berlin braucht dich! von vor zehn Jahren an. Es gibt aber hierzu auch integrationspolitisch sehr wichtige Unterschiede: Adressat sind nicht Jugendliche, sondern Betriebe bzw. die fachlich-politisch interessierte Öffentlichkeit; der Slogan »Wir zeigen: es geht« unterstreicht selbstbewusst die Fähigkeit der Betriebe, mit Vielfalt in der Ausbildung pädagogisch erfolgreich umzugehen, und der Slogan »Ausbildung: eine Frage der Einstellung «, der bewusst mehrdeutlich formuliert ist, bezieht sich mit einer seiner möglichen Bedeutungen auf das »Pilotvorhaben«.

»Einstellung« bleibt auch der Schwerpunkt der ersten Pilotvereinbarungen mit den Betrieben. Um die ersten Erfahrungen mit der Umsetzung der Vereinbarungen auszuwerten und um die weiteren Schritte zu klären, kamen auf Einladung der Senatorin für Integration, Arbeit und Soziales, Elke Breitenbach, am 7. März 2018 im Berliner Rathaus die Geschäftsführer*innen der Unternehmen mit Landesbeteiligung und Vertreter*innen der am 
Piloten beteiligten Schulen, des Kommunale Arbeitgeberverbands und Ver. di zusammen. Bemerkenswert war, dass Vertreter"innen vieler Betriebe dabei waren, was ein stabiles und sogar wachsendes Interesse an der Zusammenarbeit in Berlin braucht dich! signalisiert.

Die Geschäftsführerinnen von Ver.di und vom Kommunalen Arbeitgeberverband waren ebenfalls vertreten. Dies unterstrich das wachsende Interesse der Sozialpartner des Öffentlichen Dienstes am Erfolg von Berlin braucht dich! und insbesondere des Pilotvorhabens. Arbeitgeber wie Gewerkschaften begleiten in ihren Publikationen immer wieder durch Veröffentlichungen positiv das gesamte Vorhaben. Bildungsstaatssekretär Rackles machte durch seine aktive Teilnahme deutlich, dass Schulen wie Berufsschulen auch künftig Partnerinnen von Berlin braucht dich! sein werden.

Eine konkrete Folge dieses Treffens hätte sein können, die Vereinbarungen vor dem Hintergrund der bisherigen Erfahrungen zu präzisieren und zu erweitern. Eine zu diesem Zweck gebildete Redaktionsgruppe kam aber schließlich zu dem Ergebnis, ohne eine Erneuerung der Vereinbarungen das Pilotvorhaben weiter zu erproben. Dies wird man als Ausdruck einer abhaltenden Skepsis verstehen müssen, ob die jungen Leute, die auf diese von der bisherigen Praxis abweichende Weise in die Ausbildung gelang sind, im Ausbildungsalltag auch bestehen werden.

\section{Auf dem Weg zu neuen Schulvereinbarungen}

Im Sinne der wechselseitigen Verwiesenheit von Betrieben und Sekundarschulen in der Periode von Berufsorientierung und Vorbereitung des Übergangs von der Schule in die Ausbildung, was auch eine Verschiebung von der exklusiven Orientierung auf den Abschluss hin zu einer gleichzeitigen Orientierung auf den Anschluss bedeutet, steht 2018 auch auf der Tagesordnung, die Vereinbarungen mit den Schulen, die noch aus den Anfangsjahren von Berlin braucht dich! stammen, zu erneuern.

Es wird vorgeschlagen und soll vereinbart werden, dass die Schulen Strategien entwickeln, die dazu führen, dass Jahr für Jahr eine größere Zahl von Schulabgänger*innen in eine Ausbildung einmündet. Dass es sich hierbei um ein schwieriges Ziel handelt, kann auch daran gesehen werden, dass das durchschnittliche Alter, das junge Leute beim Eintritt in eine Berufsausbildung haben, stetig gestiegen ist und heute bei über 21 Jahren liegt. Ist ein 
direkter Übergang von der Sekundarschule in die Ausbildung ein für viele unrealistisches Ziel? Wenn sich tatsächlich in den beteiligten Schulen die Mittelgruppe derjenigen, »die Ausbildung können«, im Sinne einer ansteigenden Zahl von Übergängen stabilisieren lässt und dies das zentrale Handlungsfeld von Berlin braucht dich! ist, welche Perspektiven haben unter der Prämisse "Niemand darf zurückbleiben" die anderen Schüler*innen und welche Unterstützungsarrangements sind für diese schon vorhanden oder können gefunden werden? Das sind viele Fragen, denn zwischenzeitlich ist - auch durch zunehmend engere Kooperationsbeziehungen des BQN-Teams mit einzelnen Schulen - das Verständnis für die überaus schwierigen Verhältnisse und Rahmenbedingungen der meisten der beteiligten Sekundarschulen gewachsen und damit auch die Einsicht darin, welche Herausforderung pädagogischer, personeller und organisatorischer Art des für die Schulen ist, konstruktiv mit Berlin braucht dich! umzugehen.

Diese Erfahrungen und Einsichten hatten schon vor zwei Jahren den DGB-Landesbezirk veranlasst, gegenüber der Politik initiativ zu werden und für Sekundarschulen mit hohem Anteil von Schüler*innen ausländischer Herkunft und Lernmittelbefreiung einen erhöhten Bedarf an Berufsorientierung (»BO-Bedarfsschulen«) zu reklamieren und ein gezieltes Förderprogramm zu fordern. Tatsächlich wurde dies nun in der neuen Legislaturperiode beschlossen, aber ohne es ausdrücklich mit Berlin braucht dich! zu verknüpfen. Auch die Fixierung auf eine Verstärkung der Berufsorientierung folgt noch der mittlerweile überholten, verengten Idee eines »Allheilmittels«, während tatsächlich die sozialen Verhältnisse und Denk- und Orientierungsmuster, die die Schüler*innen vom Weg in Richtung auf eine Ausbildung abdrängen, komplexer sind und eine integrierte »Intervention" erforderlich machen. ${ }^{6}$

\section{Benachteiligung abbauen: Nur mit einem breiten Netz von Arbeitsteilung und Kooperation}

Schließlich und endlich ginge es darum, die sehr kritische Phase des z.T. länger gestreckten Übergangs von der Schule in die schließlich Einmündung in eine Ausbildung zu gestalten und die dann Auszubildenden im

6 Vgl. Kapitel 6. 
Ausbildungsprozess selbst bei Bedarf zu stabilisieren. Was das subjektive »Empowerment « betrifft, wird gegenwärtig mit »Berlin braucht dich!-Clubs experimentiert: ${ }^{7}$ Die »Clubs « richten sich an Schülerinnen und Schüler des 10. Jahrgangs in den im Konsortium mitarbeitenden Sekundarschulen. In regelmäßigen Treffen, die vom Träger BQN begleitet werden, geht es um Empowerment-Trainings, Praktika zur Unterstützung der Entscheidungsfindung, Klärung des Berufswunschs und um die Begleitung bei der Einmündung in die Ausbildung. Schüler*innen, für die ein Club »das Richtige« ist, sollen eine duale Ausbildung als Ziel haben, Neugier, Aufgewecktheit und Offenheit für Neues mitbringen und verbindlich an den Treffen teilnehmen. Bisher gibt es drei Clubs: den Club Schutz und Sicherheit, den Metallund Elektro-Club und den Gesundheitsclub.

Bei Club-Ansatz und Praxis sind noch viele Fragen offen. Der »Club« kann jedenfalls nicht die einzige Antwort auf die Problemlagen sein, die für bestimmte Gruppen von benachteiligten Jugendlichen - und unter ihnen insbesondere auch jenen aus Einwanderungsfamilien - mit dem Übergang von der Schule in Ausbildung und - später - eine stabile Beschäftigung verbunden sind. Der Aufbau eines vielgestaltigen, arbeitsteiligen, aber zugleich kooperativen und transparenten »Übergangssystems« bleibt eine noch zu lösende Aufgabe. Von daher macht es Mut, dass es erstmals in einem Bezirk, nämlich in Mitte, in den einschlägigen Bezirksausschüssen eine Debatte darüber gibt, wie auf lokaler Ebene der Ansatz von Berlin braucht dich! unterstützt werden kann.

Gegenüber dem Start von Berlin braucht dich! ist vor allem das erfahrungshaltige Problembewusstsein bei vielen Beteiligten gewachsen. Das gilt auch für den Integrationsbeauftragten. In diesem Sinne war und ist Berlin braucht dich! mit seinem Praxisprogramm eine sehr wertvolle Sonde in die Wirklich-

7 Die offizielle Beschreibung dieses Ansatzes geht so: »Der Berlin braucht dich!-Club ist eine gemeinsame Initiative von Schulen und Betrieben im Auftrag des Berliner Integrationsbeauftragten. Schüler*innen sollen auf ihrem Weg in die Ausbildung gestärkt und die Chancen auf eine erfolgreiche Berufseinmündung erhöht werden. BQN Berlin koordiniert die modellhafte Erprobung des Ansatzes mit dem Ziel, das Konzept als nachhaltiges Instrument am Übergang Schule Beruf zu etablieren. In enger Abstimmung mit den Lehrerinnen und Lehrern, den kooperierenden Betrieben und Trägern der Berufsorientierung bietet der Club Angebote zur Stärkung der individuellen Berufswahlentscheidung und Öffnung von Zugängen in betriebliche Ausbildung.«Siehe https://www.berlin-braucht-dich.de/aktivitaeten/bbd-club/ 
keit des Übergangsgeschehens in Berlin und dies insbesondere auch unter Aspekten von Integration und Teilhabe. Mittlerweile sind die komplexen $\mathrm{Zu}-$ sammenhänge von Benachteiligung und die ebenfalls komplexen Voraussetzungen und Rahmenbedingungen für ihre Aufhebung - jedenfalls im Feld der Berufsausbildung - deutlicher geworden.

Damit wird noch offenkundiger als dies schon in den vergangenen Jahren war: Ein Vorhaben wie Berlin braucht dich!, dessen Ausstattung mit Personal und ununterbrochene Laufzeit hier und da auch Neid ausgelöst haben, kann diese Herausforderung nicht bewältigen. Das gilt sowohl für die betriebliche Seite (und ihre Kooperation mit der Berufsschule) als auch für die Zusammenarbeit mit den Schulen und erst recht was die differenzierten Verfahren und Prozesse der Gestaltung der Übergänge von der Schule in die Berufsausbildung betrifft. Es ist offenkundig, dass die Hebelwirkung, die Berlin braucht dich! bereits jetzt erzeugt hat, nur dann noch wirksamer werden kann, wenn auf ausgearbeitete und funktionierende Netzwerke von Arbeitsteilung und Kooperation, insbesondere auch zur Senatsverwaltung für Bildung und zur Abteilung Arbeit aufgebaut werden kann.

Dies gilt für die Schulen im Kontext ihrer Umwelt und ihrer Kooperationspartner ebenso wie für die Betriebe, es gilt aber auch zwischen den verschiedenen zuständigen Senatsverwaltungen und innerhalb der eigenen Senatsverwaltung auch für die Zusammenarbeit mit den anderen einschlägigen Fachbereichen. Dies ist bei allen Anstrengungen und Fortschritten im Einzelnen in den vergangenen Jahren nicht mit den gesellschaftlichen Herausforderungen mitgewachsen, woran immer dies auch gelegen hat. In Hinblick auf ein breites und solides Netz von Arbeitsteilung und Kooperation gibt es erheblichen Nachholbedarf.

Mit der allmählichen Verschiebung des Maßstabs für Berlin braucht dich! auf erfolgreiche Einstiege in Ausbildung und eine erfolgreiche Bewältigung der Anforderungen in der Ausbildung kommen die Teilzeitberufsschule und die pädagogische Kooperation zwischen ausbildenden Betrieben und den ihnen korrespondierenden Berufsschulen massiv in den Blick. Die Berufsschulen müssen nun dringend als weiterer wichtiger Akteur in Berlin braucht dich! einbezogen werden.

Insgesamt wirkt das "Pilotvorhaben « wie eine Sonde, aber auch wie eine Art »Frischekur« in Hinblick auf die gesamte eingespielte Routine von Berlin braucht dich!. Alles steht erneut auf dem Prüfstand, und diesmal nicht einseitig auf der Schulseite, sondern auf der Schul- und Betriebsseite gleicherma- 
ßen, und auch beim Integrationsbeauftragten und bei $\mathrm{BQN}$ als beauftragter Agentur für Berlin braucht dich!.

\section{Fazit}

In den Kapiteln 6 und 7 wurde vertieft diskutiert, ob und wie das Entwicklungspotenzial, das betriebliche Berufsausbildung für die in sie eintretenden Auszubildenden grundsätzlich hat, auch für Jugendliche, die bisher abseits gestanden haben, genutzt werden kann. Dies waren auch die Fragen, die man sich im Konsortium, beim Träger und beim Integrationsbeauftragten stellen musste, nachdem sich herausstellte- wie in Kapitel 5 dargestellt -, dass es trotz stark verbesserter Berufsorientierung kaum Schüler*innen aus den im Konsortium mitarbeitenden Sekundarschulen gelang, bei den im Konsortium mitarbeitenden Betrieben einen Ausbildungsvertrag zu erhalten. Wichtig ist es, noch einmal festzuhalten: Die mangelnde Öffnung von Ausbildung gilt allerdings nicht für Jugendliche aus Familien mit Einwanderungsgeschichte generell; ihre Anteile wachsen in den Ausbildungen. Das Kapitel 8 schließt unter Berücksichtigung dieser Reflexion nun an das Kapitel 5 an. In der Konsortialtagung 2015 wird die geringe Zahl der Übergänge in Ausbildung zum Thema; die Betriebe kommen wieder stärker in den Blick, mit ihren Einstellungsvorbehalten und mit der Frage, ob sie bereit und in der Lage sind, das »kompensatorische Potenzial« - siehe Kapitel 7 -, das der Lernort Betrieb bietet, gezielt für Absolventen auch dieser Schulen einzusetzen. In einem ersten Schritt konzentriert sich die Aufmerksamkeit auf die betrieblichen Bewerbungs- und Auswahlverfahren, die einer kritischen Diskussion unterzogen werden.

In ersten Umrissen entsteht die Idee eines Pilotprojekts, das darauf abzielt, Bewerber*innen aus den beteiligten Schulen, die sich in den Praktika engagiert haben und eine hohe Motivation zeigen, weitgehend oder gänzlich unabhängig oder ohne die bisherigen Verfahren in die Ausbildung hineinzunehmen. Diese Idee trifft zunächst auf erhebliche Vorbehalte bei den meisten Betrieben, weil sie ihren bisherigen Verfahren zutrauen, erkennen zu können, wer voraussichtlich erfolgreich die Ausbildung absolvieren kann und wer hohe Scheiternsrisiken hat.

Dennoch erklärt sich eine Gruppe von Betrieben bereit, Ausbildungsplätze für ein solches Pilotprojekt zur Verfügung zu stellen. Diese Bereitschaft 
folgt sicherlich unterschiedlichen Motiven, ist aber mit Sicherheit auch in ihrer schon jahrelangen Mitarbeit im Berlin braucht dich!-Konsortium begründet, die eine Basis wechselseitigen Verständnisses und von Vertrauen und eine Art »Bindung« erzeugt hat, die nun erneut für einen schwierigen weiteren Schritt wirksam wird. Sich abzeichnender Fachkräftemangel, das wachsende Interesse der Sozialpartner am Vorhaben, ein bestärkter politischer Gestaltungswille und eine gute öffentliche Resonanz (Stichwort: Dem sozialen Auseinanderdriften in der Stadt entgegenwirken) sind wirksame Verstärker.

Mit dem Start des Pilotprojekts erleichterter Zugänge ohne Absenken der Anforderungen in der Ausbildung selbst wird immer wichtiger, was getan werden kann, um die erwarteten bzw. tatsächlichen Scheiternsrisiken während der Ausbildung zu verringern.

Hierbei geht es dann um drei Ansätze, deren positive Wirkung aber ihr enges Zusammenspiel erforderlich macht. Erstens geht es um eine weitere Verbesserung der in die schulische Berufsorientierung eingebetteten Betriebsbegegnungen und vor allem der Langzeitpraktika als »Erprobungsräume«, zweitens um eine auf Empowerment angelegte Begleitung nicht nur beim Übergang, sondern auch danach; die Idee der »Clubs« entsteht in diesem Kontext, und drittens um die Weiterentwicklung einer vielfaltorientierten Ausbildungsqualität. Deutlich wird, dass für die Verringerung von Scheiternsrisiken eine enge pädagogische Zusammenarbeit zwischen den Lernorten Betrieb und Berufsschule unverzichtbar ist. Die Berufsschulen bisher kaum im Blick - werden zu unverzichtbaren Partnern und müssen künftig Zugang zum Konsortium finden, was auf der übergeordneten politischen Ebene erneut die Zusammenarbeit zwischen Integrations-/Teilhabepolitik und Bildungspolitik aufruft.

Mit dem Verweis auf Qualitätsfragen der Berufsorientierung und Ausbildungsvorbereitung, der Ausbildung selbst und einer unterstützenden Begleitung wird die anfängliche Fixierung auf die Einstellungsverfahren selbst relativiert. Ihre Modifikation oder der gänzliche Verzicht auf sie wird dann vertretbar, wenn es gelingt, die erwarteten oder tatsächlichen Scheiternsrisiken in der Ausbildung wirksam zu verringern. Damit ist die aktuelle Herausforderung im Leitprojekt Berlin braucht dich! umrissen. 



\section{Kapitel 9}

\section{Nach dem "Langen Sommer des Willkommens"}

Welche Art und Weise des Umgangs Berlin mit Flüchtlingen findet, wird nicht erst im »langen Sommer des Willkommens « 2015 zum Thema. Anders als andere Bundesländer hatte der Berliner Senat Flüchtlinge als eine Zielgruppe seiner Integrationskonzepte von 2005 und 2007 benannt.

Schon seit Oktober 2012 hatten Asylbewerber*innen, von denen ein großer Teil aus afrikanischen Ländern kommt, mit verschiedenen Aktionen auf sich aufmerksam gemacht: Mit einem Camp auf dem Oranienplatz im Herbst 2012, einem Hungerstreik am Brandenburger Tor und der Besetzung der leerstehenden Gerhart-Hauptmann-Schule in Kreuzberg und einer demonstrativen Besetzung der Zentrale des DGB-Landesbezirks. ${ }^{1}$ Diese Aktivitäten sind kontinuierlich von öffentlichen Auseinandersetzungen und von Konflikten innerhalb der regierenden SPD-CDU-Koalition begleitet. Im Sommer 2014 wird durch Anweisung des Innensenators die Besetzung des Oranienplatzes beendet; in der Folge kommt es zu weiteren spektakulären Besetzungsaktionen, wie z.B. der Aussichtsplattform des Fernsehturms. ${ }^{2}$

In der Einleitung haben wir die Befürchtung formuliert, dass die Ankunft einer großen Zahl von Geflüchteten in den Jahren 2014 bis Anfang 2016 die Trends zur interkulturellen Öffnung der Berufsausbildung ein-

1 Vgl. hierzu: Hasselmann, Jörn/Frenzel, Veronica/Kneist, Sigrid/Van Bebber, Werner: »Räumung am Oranienplatz. Kein Friede den Hütten«, in: Der Tagesspiegel vom 8.4.2014.

2 Chronologie der Flüchtlingsproteste in Berlin: https://www.rbb24.de/politik/thema/fluechtlinge/hintergrund/Chronologie-Fluechtlingsproteste-Oranienplatz-BrandenburgerTor.html 
schränken könnte. Es gilt eher das Umgekehrte, dass der starke Zugang von Geflüchteten Fragen der Integrationspolitik ins Zentrum von Politik und Öffentlichkeit rückt. Im Zentrum von Bildungsaspekten stand der Zugang von Geflüchteten zu Kitas und Grundschulen; gerade für jugendliche Geflüchtete wurden Willkommensklassen an OSZ eingerichtet und der möglichst zügige Übergang in eine Ausbildung thematisiert.

Vor allem gewannen die interkulturelle Öffnung und Diversity-Kompetenz von Bezirksverwaltungen an Bedeutung. Wenngleich diese nicht unbedingt explizit angesprochen wurde, entstand recht bald auch in den Ämtern ein Konsens darüber, dass eine Metropole wie Berlin mit Zuwanderern umgehen können muss. Zum Beispiel gewann das integrationspolitische Programm der Berliner Integrationslotsinnen und -lotsen bei Kolleg*innen in den Bezirksämtern hohe Wertschätzung.

\section{Auf die vielen Geflüchteten wenig vorbereitet}

Es sollte sich zeigen, dass trotz der sich verdichtenden Vorzeichen in den unmittelbaren Jahren vor 2015 Berlin auf die Aufnahme einer großen Zahl Geflüchteter wenig vorbereitet war, und dies, obwohl im integrationspolitischen Kontext der Stadt das Thema »Flucht und Asyl« immer präsent war. Das wird bundesweit erkennbar durch die mangelnde Ausstattung des LAGeSo, des Landesamts für Gesundheit und Soziales, ${ }^{3}$ geprägt, das mit der Registrierung der Geflüchteten völlig überfordert war.

Dass dies Auswirkungen haben könnte und vermutlich würde, zeichnet sich etwa im folgenden Interview-Ausschnitt ab: »Nun sind die Flüchtlinge aber da«, sagt die Frankfurter Allgemeine Sonntagszeitung vom 30. August $2015^{4} \mathrm{zu}$ Eric Schweitzer, dem Präsidenten des Deutschen Industrie- und Handelskammertages (DIHK). Dieser antwortet: »Richtig. Und ein Viertel der Asylbewerber ist im ausbildungsfähigen Alter, zwischen 16 und 25 Jahre. [...] Es gibt ein großes Potenzial. Allerdings ist nicht jeder junge Asylbewerber [...] unmittelbar für die Ausbildung geeignet. Da gibt es unterschiedliche

3 Beikler, Sabine/Fiedler, Maria: ]etzt arbeitet die neue Behörde für Flüchtlinge, in: Tagesspiegel vom 1.8.2016.

4 https://csr-news.net/news/2015/08/30/dihk-chef-beklagt-schwierige-suche-nach-auszubildenden 
Barrieren. [...] Wir wollen von der Bundesregierung die Zusage für Asylbewerber mit Bleibeperspektive: Wer in einer Ausbildung ist, darf nicht abgeschoben werden - inklusive einer Anschlussphase von mindestens zwei Jahren im Beruf.« Darauf fragt die FAZ weiter: »Früher hieß es schnell: Bestimmte Jugendliche sind gar nicht ausbildungsfähig. Gibt es das noch oder sagen Sie: Inzwischen brauchen wir jeden?« Und Schweitzer antwortet: »Wir bemühen uns um jeden. Aber es bleibt leider immer noch ein Teil, den wir nicht vermitteln können. [...] Dazu kommt, dass die Anforderungen in vielen Ausbildungsberufen stark gestiegen sind.«

Das Bild ändert sich aber im Laufe der nächsten Monate mit positiven Berichten von Ausbildungserfolgen. Insgesamt zeigte sich in Hinblick auf Erfahrungen mit Schule und Bildung und Bildungsverhalten unter den jungen Geflüchteten eine ähnliche »Dreiteilung«, wie wir sie auch bei uns kennen. Es gibt gut Vorgebildete, Leistungs- und Anpassungsfähige, die ihren Weg gehen - aber eher in ein Studium als in eine Ausbildung streben. Eine mittlere Gruppe hat gute Chancen auf lebbare berufliche Perspektiven, wenn ihre besondere Ausgangslage und die besondere Verletzlichkeit ihrer Motivationen im Bildungssystem Beachtung finden. Schließlich wird es eine nicht kleine dritte Gruppe von Jugendlichen geben, die seit Monaten oder Jahren keine Schule besucht oder überhaupt nur eine unzureichende formale Bildung hat, aber auch jene, die durch die Situation im Herkunftsland, durch die Flucht und ihr Ankommen hier physisch, psychisch oder moralisch besonders angegriffen sind.

Das Stichwort heißt hier: Prekäre Lebensverhältnisse. Die jungen Geflüchteten der dritten Gruppe werden die Zahl derjenigen jungen Leute verstärken, die hier aufgewachsen sind - aus Familien mit oder ohne Migrationsgeschichte - und sich in erheblichen Risikolagen befinden, was ihre berufliche Zukunft und eine eigenständige Lebensführung betrifft. Einer solchen Entwicklung ist dringlich entgegenzutreten - mit allen Instrumenten der Bildungs- und Integrationspolitik sowie den zur Partizipation und Integration erheblich verstärkten Mitteln der Bezirke. 


\section{Schritte und Bausteine für Integration und Teilhabe}

Insbesondere in Hinblick auf junge Geflüchtete werden sofort viele Maßnahmen initiiert. Im Verband der Berliner Metall- und Elektroindustrie - Partner von Berlin braucht dich! - z.B. werden Überlegungen zu einem Coaching von geflüchteten Kindern entwickelt. Der Träger Arbeit und Bildung, seit 2006 mit berufsbezogener Beratung des »Kumulus- Projekts« am Oberstufenzentrum Körperpflege präsent, erweitert die Schulsozialarbeit ab September 2015 durch Sofortmaßnahme für Geflüchtete in den Willkommensklassen der OSZ-Versorgungstechnik-Max-Taut-Schule, Logistik-Touristik-Steuern-LOTIS, OSZ-Wirtschaft und Sozialversicherung-WISO-Hermann-Scheer-Schule, OSZ-Technische-Informatik-Energietechnik-TIEM. Auch im Rahmen der Jugendverbandsarbeit werden umgehend Unterstützungsvorhaben aufgelegt. ${ }^{5}$

Schließlich einigen sich die wichtigsten landesweiten Akteure 2016 auf ein Berliner 10-Punkte-Programm und bilden hierzu auch eine koordinierende Struktur:

In Berlin wirken alle wichtigen Arbeitsmarktakteure in der Lenkungsgruppe »Arbeitsmarktintegration Geflüchteter« zusammen. Dazu gehören die Regionaldirektion Berlin-Brandenburg der Bundesagentur für Arbeit (RD BB), die Industrie- und Handelskammer (IHK), die Handwerkskammer Berlin (HWK), der Deutsche Cewerkschaftsbund Berlin-Brandenburg (DCB), die Unternehmensverbände Berlin-Brandenburg e.V. (UVB), die Spitzenverbände der freien Wohlfahrtspflege (LICA) sowie verschiedene Senatsverwaltungen. Hier werden sämtliche Aktivitäten koordiniert und zusammengeführt. Eine Koordinierungsstelle beim Integrationsbeauftragten wird die Arbeit der Steuerungsgruppe begleiten. Es geht um die Bündelung von spezifischen Maßnahmen für geflüchtete Menschen, Öffnung von vorhandenen Förderinstrumenten und Verknüpfung der verschiedenen Aktivitäten. ${ }^{6}$

Das Amt des Integrationsbeauftragten kommt hier also in einer koordinierenden Funktion ins Spiel, Seit Oktober 2015 ist Andreas Germershausen -

\footnotetext{
5 www.aub-berlin.de/fuer-jugendliche/schulsozialarbeit-an-den-berliner-oberstufenzentren/

6 https://www.berlin.de/sen/arbeit/berlinarbeit-ziel-4/arbeitsmarktintegration/
} 
seit 2001 dort beschäftigt - Beauftragter des Berliner Senats für Integration und Migration und damit auch Leiter der Abteilung Integration in der Senatsverwaltung für Integration, Arbeit und Soziales. Seine Arbeit in diesen Monaten wird vor allem durch zwei große Projekte bestimmt, die der dem Feld»Geflüchtete« Struktur und Kontinuität geben sollen: dem »Masterplan Integration und Sicherheit«, der am 24.5.2016 verabschiedet wird, ${ }^{7}$ und der Eröffnung des Willkommenzentrums in der Potsdamer Straße im August 2016. ${ }^{8}$ Zugleich und mit dem "push« des Flüchtlingsgeschehens verbessert sich auch moderat die Personalsituation beim Integrationsbeauftragten.

\section{Das gesellschaftliche Klima wird rauher}

Zugleich wird das gesellschaftliche Klima rauher. Die verspätete politische Einsicht, dass Deutschland ein Einwanderungsland ist und bleibt, wirft nun - durch die Aktualisierung, die diese Frage durch den »Flüchtlingssommer" erhalten hat - lange Schatten. Schon 1988 hatten Andreas Germershausen und Wolf-Dieter Narr auf die negativen Folgen einer solchen Verleugnung hingewiesen: »Nichts kennzeichnet die Stärke schlimmer deutscher Vergangenheit so sehr, nichts bezeichnet so nachhaltig die innere, die substantielle Schwäche der Bundesrepublik, wie das menschenabstoßende Dauermotto 'Die Bundesrepublik ist kein Einwanderungsland . Die Politik der Schließung, widersprüchlich zur internationalen Abhängigkeit der Bundesrepublik, wirkt nicht nur negativ nach außen, sie wirkt gleicherweise repressiv, Freiheit und Pluralität raubend, nach innen. ${ }^{9}$ Wüsste man nicht, dass dieses Statement aus dem Jahr 1988 stammt, könnte man es fast als einen Kommentar zu den politischen Debatten des Jahres 2018 lesen.

Pegida (Akronym der selbstgewählten Bezeichnung »Patriotische Europäer gegen die Islamisierung des Abendlandes«) wird schon im Dezember 2014 gegründet und gelingt eine erste große Demonstration in Dresden im Januar 2015. Ein weiterer Höhepunkt der Mobilisierung ist der Oktober 2015. Aber

\footnotetext{
7 https://www.berlin.de/fluechtlinge/infos-zu-fluechtlingen/masterplan/

8 Benalia, Emina: Neues Willkommenszentrum in Berlin nimmt seine Arbeit auf, in: Berliner Morgenpost vom 18.08.2016.

9 Germershausen, Andreas/Narr, Wolf-Dieter: Flucht und Asyl in der Politik der Bundesrepublik, Berlin 1988, S. 23-34.
} 
dies ist kein ausschließlich Dresdener oder ostdeutsches Phänomen, wie sich schnell zeigen sollte. In Berlin z.B. finden nach dem Sommer 2015 wöchentlich rechte Protestaktionen statt. Die rechte Gewalt ging nach Angaben von ReachOut, Beratungsstelle für Opfer rechter, rassistischer und antisemitischer Gewalt in Berlin, in 2016 in Berlin gegenüber 2015 zwar etwas zurück, lag aber deutlich über dem Niveau von 2014. ${ }^{10}$ Immer wieder gab es und gibt es Angriffe auf Moscheen und antisemitische Übergriffe. Die Vorgänge der Kölner Silvesternacht $2015^{11}$ sind ein weiterer Treibsatz für die Formierung einer pointiert antimuslimischen, aber insgesamt gegen Einwanderung gerichteten, völkisch grundierten Politik. Damit ist auch die Aufklärungsaufgabe des Integrationsbeauftragten erneut aufgerufen.

\section{Das Schulsystem bleibt eine "große Baustelle»}

Angesichts dieser Gesamtsituation steht auch das strukturell reformierte Berliner Schulsystem vor einer erneuten Bewährungsprobe. Die Antwort auf die große Zahl geflüchteter Kinder und Jugendlicher war zunächst die Einrichtung von "Willkommensklassen« und eine neue Leitlinie für die berufliche Integration der über 16-jährigen Jugendlichen. ${ }^{12}$

Nach der Wahl zum Abgeordnetenhaus im September 2016 wird ein neuer Senat aus SPD, Linken und Grünen gebildet. Diese neue Koalition geht im folgenden Jahr auf Distanz zur bisherigen zentralen Beschulung von jungen Geflüchteten. Rund vier Millionen Euro sollen investiert werden, um die schulische Situation junger Geflüchteter wie auch abschlussgefährdeter Schüler*innen zu verbessern u.a. durch weiteren Ausbau der Schul-

10 https://www.reachoutberlin.de/de/chronikReachout

11 In der Silvesternacht 2015 hatten offenkundig mehrere Migranten sexuelle Gewalt gegenüber deutschen Frauen ausgeübt. Dieser Vorfall wurde auf die Aufnahme von Geflüchteten bezogen, obgleich die Vorfälle wohl von nordafrikanischen Migranten ausgingen, die schon lange in der Bundesrepublik lebten. Seit der Silvesternacht wurde verstärkt gefordert, Geflüchteten die Werte des Grundgesetzes zu vermitteln. Der Vorfall hat mit dazu geführt, dass der Berliner Senat seinen 2016 verabschiedeten Masterplan zur Integration Geflüchteter um den Begriff der Sicherheit ergänzte.

12 Vgl. Beiträge von Ralf Wiechert-Beyerhaus auf dem JahresforumExtra im Dezember 2016, www.kommunale-koordinierung.de/files/Jahresforen/Jahresforum_Extra_2016_Berlin/ Dokumentation/Forum2_Ralf_Wiechert-Beyerhaus_Folien.pdf 
sozialarbeit. Außerdem erhalten einige sozial besonders schwierige Schulen Mittel aus einem »Brennpunktprogramm« des Senats und aus dem »Turnaround«-Förderprogramm. Tatsächlich hat die Strukturreform mit dem Kernstück der Integrierten Sekundarschule die Bildungschancen insbesondere der Kinder und Jugendlichen mit erheblichen Scheiternsrisiken nicht verbessern können. Der Tagesspiegel resümiert:

Nur 62 Prozent aller Zehntklässler der Sekundarschulen haben im Schuljahr 2016/17 den Mittleren Schulabschluss geschafft-drei Prozent weniger als im Jahr zuvor. Jeder Zehnte verlässt die Sekundarschule ohne Abschluss. Dafür steigt die Quote der Schulabbrecher: Jeder Zehnte verlässt die Sekundarschule ohne Abschluss - doppelt so viele Schüler wie im Bundesdurchschnitt. Die Ergebnisse von Jugendlichen aus Migrantenfamilien haben sich sogar noch weiterverschlechtert: Von ihnen erreichen 16 Prozent keinen Schulabschluss - vier Prozentpunkte mehr als noch 2012. ${ }^{13}$

Die Risiken einer verstärkten sozialen Spaltung der Stadt wachsen also gewissermaßen weiter nach. ${ }^{14}$ Wie aktuell dieses Thema ist, zeigt auch eine neue Studie des Wissenschaftszentrums für Sozialforschung. ${ }^{15}$

\section{Die Herausforderung "Geflüchtete«: ein Schub für die Integrationspolitik?}

Es wäre also sehr problematisch gewesen, wenn die »Flüchtlingsfrage« die grundständigen Probleme sozialer Ungleichheit und die Frage nach ihrer Bekämpfung verdrängt hätten. Das war aber - mit der Ausnahme einer starken öffentlichen und politischen Dominanz in der Zeit direkt nach dem »Flücht-

13 Nowakowski, Gerd: Berliner Schulpolitik. Das Ergebnis der Bildungspolitik ist verheerend, in: Tagespiegel vom 25.6.2018. Dieser Bericht bezieht sich auf die Ergebnisse der »Berlin-Studie«, die von Jürgen Baumert vom Max-Planck-Institut für Bildungsforschung geleitet und vom Land Berlin und der Jacobs-Foundation gefördert wurde.

14 Vgl. u.a.: Butler, Jeffrey/Odenwald, Stephanie/Bochum, Ulrich/Kohlmeyer, Klaus: Soziale Spaltungen in Berlin, Hamburg 2016.

15 Helbig, Marcel/]ähnen, Stefanie: Wie brüchig ist die soziale Architektur unserer Städte? Trends und Analysen der Segregation in 74 deutschen Städten (=Discussion Paper P 2018o01), Berlin 2018. 
lingssommer « - nicht der Fall. Fast schon umgekehrt kann gesagt werden, dass sich mit dem »Schub«, der durch die Bewältigung der Zuwanderung einer Vielzahl von Migrant"innen - Geflüchteten, aber auch Zuwanderung aus den südosteuropäischen Ländern, auch Polen und aus den südeuropäischen Ländern, die besonders stark von der Finanzkrise 2008 betroffen waren -, entstand, die Voraussetzungen für Teilhabepolitik eher verbessert haben.

Dies lässt sich nicht nur daran festmachen, dass die Frage, was das Schulsystem leistet, nicht mehr von der Tagesordnung genommen wurde, sondern auch daran, dass die Aufnahme der Geflüchteten eine Art »Lernstück« dafür war und ist, wie Institutionen und Kooperationen besser aufgestellt werden können. Das gilt zum einen für das Amt des Integrationsbeauftragten selbst, zum anderen aber vor allem auch in der dialogischen Art und Weise, wie das Nachfolgekonzept zum »Plan für Integration und Sicherheit« erarbeitet wurde. Dazu wurden gemeinsam mit den Bezirken sowie der Zivilgesellschaft Lebensrealität und -bedingungen Geflüchteter analysiert und auf dieser Basis konkrete Ziele zur Verbesserung der Situation Geflüchteter in den Folgejahren definiert. Am Prozess sind auch Geflüchtete beteiligt. Zielgruppe der Lösungsstrategien sind alle in Berlin lebenden Geflüchteten unabhängig von ihrem Herkunftsland. Das Gesamtkonzept wird in neun Handlungsfeldern erarbeitet. Diese orientieren sich an den Lebensbereichen der Geflüchteten.

Charakteristisch für die dialogische Vorgehensweise ist "Integration im Dialog«, eine Gesprächsreihe in allen Bezirken mit dem Berliner Integrationsbeauftragten zusammen mit Bezirksbürgermeister"innen, Vertreter*innen der Bezirksämter, Vereinen, Initiativen, Geflüchteten und den Bürgerinnen und Bürgern der Berliner Bezirke.

\section{Bezirke}

Ein wichtiger Effekt dieser Vorgehensweise ist, dass damit die Rolle der Bezirke in der Integrations- und Teilhabepolitik weiter gestärkt wird. Eine Reihe von ihnen mit ihren jeweiligen Integrationsbeauftragten befand sich ohnehin schon seit Jahren auf dem Weg zu einer größeren Initiative - und Verantwortungsübernahme im Gesamtfeld von Einwanderung, trotz geringen rechtlichen Kompetenzen und der schwachen Ausstattung. Als ein Beispiel soll hier der Bezirk Pankow genannt werden, der sich mit einer Studie schon früh vergewisserte, wie sich dort Einwanderung sozial und sozial- 
räumlich darstellt, ${ }^{16}$ und ein enges Kooperationsnetzwerk entwickelt hat Als ein weiteres Beispiel soll der Bezirk Mitte genannt werden, der zum einen in der Geflüchtetenarbeit aktiv ist, zum anderen aber in der Bezirksverordnetenversammlung vom Mai 2018 beschlossen hat, alle Sekundarschulen des Bezirks aufzufordern, in Berlin braucht dich! mitzuarbeiten und hierfür auch eine bezirkliche Koordinierung angedacht hat.

Die integrations- und teilhabepolitische Verantwortung der Bezirke zu stärken, wäre auch eine Konsequenz aus dem "Schlussfolgerungen und Empfehlungen« des Berichts zur Jugendgewalt des Berliner Forums für Gewaltprävention. ${ }^{17}$ Auf der bezirklichen Ebene - mehr noch als auf der Gesamtberlins - entwickelte sich ein erhebliches Engagement von Willkommensbündnissen und Migrantenorganisationen in der Flüchtlingsarbeit. Der SPD-CDU Senat hat durch seinen Masterplan (Mai 2016) Nichtregierungsorganisationen in den Bezirken finanziell erheblich gefördert. Der rotrot-grüne Senat hat das mit seinem Gesamtkonzept zur Partizipation und Integration Geflüchteter (Dezember 2018) fortgesetzt.

\section{Das Prinzip "sowohl - als auch"}

Ein wichtiges Indiz dafür, dass seitens der Integrationspolitik darauf geachtet wird, dass die Rechte und Interessen derjenigen jungen Leute aus Familien mit Migrationsgeschichte, die in Berlin geboren und aufgewachsen sind, nicht vergessen werden, ist die kontinuierliche Fortsetzung des Leitprojekts Berlin braucht dich!. Zwar gab es 2015 kurzfristig die Überlegung, Berlin braucht dich! auch in Hinblick auf junge Geflüchtete in besonderer Weise in Stellung zu bringen. Das wurde aber nicht weiterverfolgt, um weder Kapazitäten noch Aufmerksamkeit von der Kernaufgabe von Berlin braucht dich! abzuziehen.

Anfang 2016, also in einer Zeit, in der die »Flüchtlingsfrage« alles zu dominieren schien, äußert sich der Integrationsbeauftragte pointiert - in einem internen Positionspapier von AL III vom 25.2.2016 - zur Notwendigkeit, nun tatsächlich auch die Ausbildung für jene zu öffnen, die bisher kaum eine Chance hatten:

16 Aumüller, Jutta : Vielfalt in Pankow. Die Beteiligung von MigrantInnen auf lokaler Ebene, Berlin 2014.

17 https://www.berlin.de/lb/lkbgg/publikationen/berliner-forum-gewaltpraevention 
Es geht um Jugendliche, für die Berufsausbildung die weit und breit einzige halbwegs realistische Perspektive für einen Weg in stabile Erwerbstätigkeit ist. Scheitern viele an dieser Stelle, dann wächst die Gruppe jener Jugendlichen in Risikolagen, die sich von der Gesellschaft abgehängt fühlen - mit unkalkulierbaren Auswirkungen auf die Stadtgesellschaft. Die Öffnung beruflicher Perspektiven für junge Geflüchtete ist eine sehr aktuelle und große Herausforderung. Darüber dürfen aber nicht jene weniger Beachtung finden, die hier geboren und aufgewachsen sind. Es geht also nicht um ein sentweder-oder«, sondern um ein integrationspolitisch gebotenes ssowohl-als-auch .

\section{Gewerkschaften}

Auch die Gewerkschaften beharren darauf, dass die Jugendlichen mit erheblichen Scheiternsrisiken beim Weg von der Schule in den Beruf nicht nur nicht vergessen werden, sondern in besonderer Weise Unterstützung finden. So wendet sich - wie bereits erwähnt - die damalige Vorsitzende des DGB-Landesbezirks Berlin-Brandenburg, Doro Zinke, im Februar 2016 mit einem Brief an die Bildungssenatorin und an die Bildungspolitiker*innen der Fraktionen, in denen gezielte Unterstützung der Berufsorientierung in jenen Schulen gefordert wird, die zwei in den offiziellen Statistiken benutzte Merkmale haben, nämlich einen sehr hohen Anteil von Schüler*innen ausländischer Herkunft und zugleich einen sehr hohen Anteil von Lernmittelbefreiung, was ein Hinweis auf familiären SGB-II-Bezug ist.

Zwar betont der Bildungsstaatssekretär in seinem Antwortschreiben vom März 2016, dass Berlin mit Berufsorientierung gut dastehe; aber der geforderte Ansatz findet sich im Sonderfinanzierungspaket der Bildungsverwaltung vom Jahr 2017 wieder. Dort ist dann der Träger Teach First vorgesehen, der junge Hochschulabsolventen als >fellows`zur Unterstützung in sogenannten >Brennpunktschulen einsetzt, allerdings ohne gleichzeitig eine Zusammenarbeit mit dem Integrationsbeauftragten und mit Berlin braucht dich! verbindlich zu machen.

Der DGB-Landesbezirk bekräftigt auf seiner Konferenz im Januar 2018, auf der Christian Hoßbach als neuer Vorsitzender gewählt wird, mit der Annahme einer aktualisierten Version des IGM-Antrags von 2014 seine Forderung nach einem landesweiten integrativen Übergangssystem, unter Ein- 
schluss der Jugendberufsagenturen, die die bezirkliche Komponente eines solchen Systems verstärken könnten.

Zum »Stand des Aufbaus und der Arbeit der Jugendberufsagenturen« findet im Mai 2017 eine Anhörung im Abgeordnetenhausstatt - mit einer durchmischten Bilanz. ${ }^{18}$ Die Jugendberufsagentur Berlin ist ein durch das Land Berlin (mit den für Bildung, Jugend und Arbeit zuständigen Senatsverwaltungen sowie den Jugendämtern/Bezirksämtern), die Jobcenter und die Agenturen für Arbeit getragenes Arbeitsbündnis mit jeweils einem gemeinsamen Standort in jedem Bezirk.

Das übergreifende Ziel der Jugendberufsagenturen ist es, jeden Berliner Jugendlichen oder jungen Erwachsenen, der in der Regel das 25. Lebensjahr noch nicht vollendet hat, zu einem Berufsabschluss zu führen. Dies soll so geschehen, dass er/sie umfassend und ggf. aufsuchend beraten wird, seine/ ihre Zielperspektiven geklärt werden, ihm/ihr ein realistisches Qualifizierungsangebot unterbreitet wird, flankierende Maßnahmen gebündelt werden, er/sie bis zum erfolgreichen Ausbildungsabschluss oder im Einzelfall einer nachhaltigen Beschäftigungsaufnahme unter Einbeziehung aller zur Verfügung stehenden Instrumente begleitet wird, wenn es erforderlich ist.

Damit agieren die Jugendberufsagenturen - wie auch Berlin braucht dich! - im Feld des Übergangs von der Schule in die Arbeitswelt. Was Berlin braucht dich! vor allem von den Jugendberufsagenturen unterscheidet, ist sein konsortialer Ansatz, also die enge und kontinuierliche Zusammenarbeit einer Gruppe von Sekundarschulen mit einer Gruppe von Ausbildungsbetrieben.

Diesem modellhaften und sich die langfristige Förderung beruflicher Perspektiven von Jugendlichen in innerstädtischen Sekundarschulen beziehende Ansatz steht eine auf die Gesamtheit aller Unterstützung bedürftigen jungen Menschen orientierter Ansatz der Jugendberufsagenturen gegenüber. Während der Ansatz von Berlin braucht dich! explizit integrations- und teilhabepolitisch ausgerichtet ist, bleibt die Verankerung des »Fokus Migration« bei den Jugendberufsagenturen noch unscharf. ${ }^{19}$ Eine Verknüpfung der beiden (und anderer im Übergang Schule - Arbeitswelt agierender) Ansätze erfolgt bisher nicht, ist aber sinnvoll und müsste im Rahmen der weiteren Gestaltung eines Berliner Übergangssystemsaufgegriffen werden. ${ }^{20}$

\footnotetext{
18 https://www.parlament-berlin.de/ados/18/IntArbSoz/protokoll/ias18-012-wp.pdf 19 Vgl. Kapitel 2. 20 Vgl. Kapitel 10.
} 


\section{Die Nachwuchslücke wird immer krasser}

Schließlich wächst dem Leitprojekt Berlin braucht dich! auch von der immer deutlicher werdenden Nachwuchslücke weiter potenzielle Wichtigkeit zu. "Durch den jahrelangen Sparkurs ist die Berliner Verwaltung inzwischen hoffnungslos überaltert. Mehr als ein Viertel der Landesbeschäftigten wird bis zum Jahr 2019 in den Ruhestand gehen. In den kommenden zwei Jahren müssen deshalb knapp 25.000 Stellen neu besetzt werden. Insgesamt gibt es aktuell rund 113.000 Beschäftigte in der Verwaltung - darunter allein 16.500 Polizistinnen und Polizisten, 5.300 Feuerwehrleute, 20.500 Beschäftigte im allgemeinen Verwaltungsdienst, 28.400 Lehrer, 8.100 Mitarbeiter im Justizvollzugsdienst und 6.200 Fachkräfte in der Steuerverwaltung. Und nahezu in allen Bereichen fehlt es an geeigneten Bewerbern«, resümiert die Berliner Morgenpost in einem Beitrag über den Plan des Berliner Senats, in einer großangelegten, professionellen Kampagne gezielt um Nachwuchs- und Führungskräfte zu werben. ${ }^{21}$ Der Nachwuchsmangel ist so groß, dass z.B. der Innensenat prüft, die Bestimmung über die Mindestgröße von Polizisten, bisher $160 \mathrm{~cm}$, außer Kraft zu setzen. ${ }^{22}$

Vor dem Hintergrund der Gesamtentwicklungen der vergangenen Jahre sind nun erneut Gespräche mit der Arbeits- und Bildungsverwaltung und mit der Innensenatorischen Verwaltung im Gang, um durch die Verbesserungen der Kooperationsbeziehungen auch die integrations- und teilhabepolitischen Wirkungen weiter zu verstärken. Denn der Zugewinn an Vielfalt mit heute 27,2 Prozent jungen Leuten aus Familien mit Migrationsgeschichte im Durchschnitt bei neu eingestellten Auszubildenden im Öffentlichen Dienst und in den Betrieben mit Landesbeteiligung ${ }^{23}$ und das beachtliche Gesamtpanorama der Investition in Integrationspolitik - 42 Millionen Euro $-{ }^{24} \mathrm{kann}$ nicht über die fortbestehenden Defizite und ihre teilweise auch drohende Verschärfung hinwegtäuschen.

21 Berliner Morgenpost vom 15.7.2017.

22 Berliner Morgenpost vom 17.5.2018 und: Zawatka-Gerlach, Ulrich: 15 Prozent offene Stellen. In der Berliner Verwaltung fehlt massiv Personal, in: Tagespiegel vom 4.1.2018.

23 Pressemitteilung des Integrationsbeauftragten vom 30.5.2018.

24 Der Beauftragte des Berliner Senats für Integration und Migration: Berliner Senat investiert 42 Millionen in die Integrationspolitik, Pressemitteilung vom 21.6.2018. 
Abbildung 8: Entwicklung der Anteile neu eingestellter Auszubildender mit Migrationshintergrund

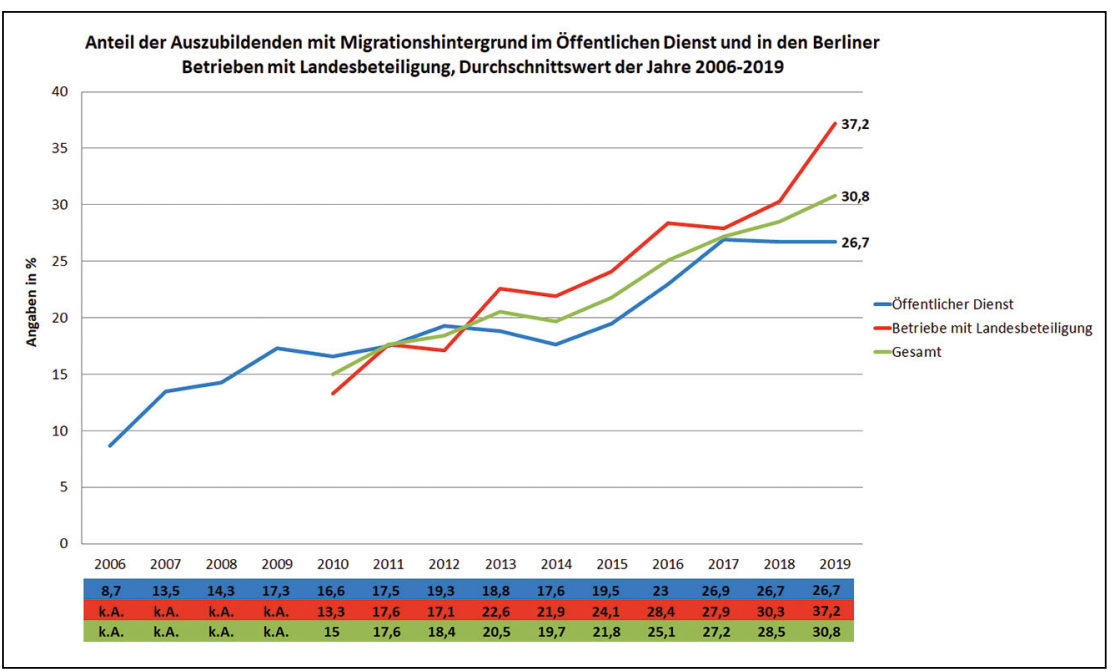

Copyright: BQN Berlin

\section{"Wir sind in der Realität schon weiter»}

Zugleich wird angesichts der öffentlichen Debatten auch im Sommer 2018, in denen unter anderem durch den Innenminister der Bundesrepublik Deutschland geäußert wird, die »Migration sei die Mutter aller Probleme«, Information und Aufklärung erneut zu einer wichtigen Aufgabe, vor allem auch dann, wenn mit einigem Recht gesagt werden kann: »Wir sind in der Realität schon viel weiter als diese Debatten, die den Nährboden für Rechtspopulisten bilden können. $\ll^{25}$

25 Beikler, Sabine: „Wir sind in der Realität schon viel weiter«. Berlins Integrationsbeauftragter Andreas Cermershausen im Interview über Rassismus, Heimatgefühle und Debatten, die Populisten helfen. In: Tagesspiegel vom 30.7.2018. 


\section{Fazit}

Das Kapitel 9 zeichnet eine Zwischenbilanz des integrationspolitischen Leitprojekts Berlin braucht dich! nach, die das Erfordernis einer Re-Orientierung auf die Tagesordnung setzte. Damit verbunden war eine integrations- und teilhabepolitische Schärfung der Problemlage; pointiert formuliert: gerade diejenigen jungen Menschen aus Familien mit Einwanderungsgeschichte, für die eine Berufsausbildung besonders wichtig wäre, bleiben von ihr ausgeschlossen. Die Diskussion der Gründe und Zusammenhänge, die zu einer solchen Lage führen, wie sie vor allem in den Kapiteln 6 und 7 skizziert wurde, ließ einen erheblichen Handlungsbedarf deutlich werden: eine große integrations-, aber auch bildungs- und arbeitsmarktpolitische Baustelle.

Welches Gewicht oder welche Bedeutung dieser Baustelle zugewiesen wird, hängt auch von der integrationspolitischen Gesamtlandschaft $a b$, wie sie sich in den Jahren 2014 folgende darstellt. Dies ist das Thema des neunten Kapitels; es nimmt dabei gewissermaßen den »roten Faden« aus dem dritten Kapitel wieder auf, das mit dem Hinweis auf das wachsende Gewicht der »Flüchtlingsfrage« endete.

Tatsächlich wird die »Flüchtlingsfrage« und hierin insbesondere auch die Situation junger Geflüchteter zu einer großen Herausforderung und $\mathrm{zu}$ einem der zentralen Arbeitsschwerpunkte des Integrationsbeauftragten, wie z.B. eine Koordinierungsgruppe für das Zehn-Punkte-Programm zur Arbeitsmarktintegration Geflüchteter und die Federführung für den Masterplan »Sicherheit und Integration« aus dem Jahr 2016.

Es zeigt sich rasch, dass der insbesondere von der Wirtschaft erhoffte Kompetenz-Schub durch gut vorgebildete und hoch motivierte junge Geflüchtete nur zu einem begrenzten Teil der Realität entsprach; viele andere aber aufgrund der Lage in ihren Herkunftsländern und der langen und belastenden Flucht eher zu jenen gezählt werden müssen, die mit erheblichen Schwierigkeiten zu tun haben und in Hinblick auf die Integration in Ausbildung und Arbeit eher zu den Risikogruppen zählen.

Diese Risikogruppen differenzieren sich nun aus: $\mathrm{Zu}$ den Jugendlichen aus Familien mit Einwanderungsgeschichte, die in Berlin geboren oder aufgewachsen sind - eine zentrale Zielgruppe von Berlin braucht dich! - treten nun auch zahlreiche Menschen aus der Gruppe der neu angekommenen jungen Geflüchteten. Es werden vielfältige Anstrengungen unternommen, den jungen Geflüchteten einen Weg zu schulischer und beruflicher Bildung 
bahnen zu helfen; es entstehen bezirkliche Netzwerke und Projekte zu ihrer lebensweltlichen Unterstützung. Zugleich setzt die Berliner Integrationspolitik ihre strategische Option zur Öffnung der Berufsausbildung und damit auch das Leitprojekt in diesem Feld konsequent fort; »sowohl - als auch" ist hierbei die Orientierung.

Das Ankommen einer großen Zahl von jungen Geflüchteten stellt das Berliner Schul- und Ausbildungssystem und die Integrationspolitik vor erhebliche neue Herausforderungen; zugleich wird das gesellschaftliche Klima mit dem Aufkommen rechtspopulistischer politischer Kräfte rauher. Dies führte aber nicht zu einem pragmatischen »muddling through", sondern war offenbar Anstoß für eine kritische Befragung der bisherigen Verhältnisse, z.B. im Feld der Schule, und für die Suche nach Ansätzen, die auch in Zukunft tragfähig sind.

So entstehen auf der bezirklichen Ebene neue Arbeitszusammenhänge, an die der Integrationsbeauftragte bei der partizipativen Entwicklung des Nachfolgekonzepts zum Plan für Sicherheit und Integration anknüpfen konnte. Migrantenorganisationen engagieren sich sichtbar im Feld der Arbeit mit Geflüchteten; der DGB Landesbezirk weist auf die schwierige Lage an den innerstädtischen Integrierten Sekundarschulen hin, die zuständige Senatorin unterstützt nach Kräften den - im vorherigen Kapitel beschrieben - aus der Re-Orientierung von Berlin braucht dich! entstandenen Pilotansatz.

Die 2010er Jahre brachten - so wurde im Kapitel 3 beschrieben - eine Aufbruch-Stimmung, die immerhin in eine erhebliche Stärkung von Berufsorientierung mündete. Die wesentlich schwierige, vielgestaltige und drängende Problemlage der Jahre ab 2016 zwingt dazu, nach "systemischeren" Antworten auf die Herausforderungen sozialer Ungleichheit zu suchen. Dies könnte auch für den Übergang Schule - Beruf gelten. Das nächste abschließende Kapitel greift diese Frage noch einmal auf. Im integrationspolitischen Feld der Öffnung der Berufsausbildung bieten sich Chancen zur Einstellung von Migrant*innen, da der Öffentliche Dienst in naher Zukunft auf eine erhebliche Fachkräftelücke zusteuert. Berlin braucht dich! hat nun nicht nur politischen, sondern auch erheblichen personalwirtschaftlichen Gehalt. 



\section{Kapitel 10}

\section{Ausblick: Übergänge insgesamt in den Blick nehmen}

In den bisherigen Kapiteln ging es vor allem um die Frage nach der (stärkeren) Öffnung von Berufsausbildung für junge Menschen aus Familien mit Migrationsgeschichte. Die schon Jahrzehnte in immer neuen Auflagen geführte Diskussion unter dem Schlagwort »Übergangssystem《 war stets besonders stark mit der Frage verbunden, wie die Einmündungen in eine Berufsausbildung erfolgen. ${ }^{1}$ Sie war vor allem im Zuge der »Berufsnot« von Jugendlichen, also dem hinter der Nachfrage stark zurückbleibenden Angebot an Ausbildungsplätzen entstanden, ${ }^{2}$ um sich später unter dem Aspekt der Benachteiligung vor allem auch Jugendlichen mit Migrationsgeschichte zuzuwenden.

Im Zentrum stand dabei stets die duale Berufsausbildung, in der ausbildende Betriebe eine dominante Rolle spielen. Zwar erscheint zuweilen in Formulierungen wie »Übergang Schule - Arbeitswelt« der Horizont weiter

1 Aufgrund der traditionellen Fixierung auf die Berufsausbildung als einem der »Königswege« wird das in Zeiten mangelnder Ausbildungsplätze entstandene Bündel von Maßnahmen lange Zeit als »Übergangssystem « definiert. Aber schon früh gibt es auch Vorschläge, in einem erweiterten Verständnis als Übergangssystem alle jene Ausbildungsgänge und Maßnahmen zu verstehen, die zwischen der Schule und dem Eintritt in ein qualifiziertes Arbeitsverhältnis angesiedelt sind: Brock, Ditmar/Hantsche, Brigitte/Kühnlein, Gertrud/ Meulemann, Heiner/Schober, Karen (Hg.): Übergänge in den Beruf. Zwischenbilanz zum Forschungsstand, München 1991; vgl. hierzu auch Kruse, Wilfried/Expertengruppe: Jugend. Von der Schule in die Arbeitswelt, Stuttgart 2010.

2 Ein Begriff, der in der unmittelbaren Nachkriegszeit geprägt wurde, und auch später immer dann Verwendung fand, wenn einer hohen Nachfrage nach Ausbildungsplätzen ein deutlich geringeres Angebot gegenüberstand. 
gefasst; de facto aber ist die Rede zumeist von einem bestimmten Ausschnitt der Übergänge in das Arbeitsleben, nämlich eben jenen, die sich auf das betriebsbasierte »Duale System « beziehen.

Die Rede vom »Übergangssystem « bekam zudem mit der Veränderung der Vorzeichen auf dem Ausbildungsmarkt, also dem wachsenden Ruf der Betriebe nach Bewerber*innen, einen abwertenden Beigeschmack, weil darunter vor allem die große Anzahl von berufsvorbereitenden Maßnahmen verstanden wurden, die nun als eine Art Hinderung für den »direkten Weg in die Ausbildung « betrachtet werden. ${ }^{3}$ Die Folge ist, dass sie stärker aus dem Blickfeld geraten sind, und damit auch ihr oftmals erhebliches pädagogisches Potenzial.

\section{Blick auf duale Ausbildung integrationspolitisch wichtig, aber zu eng}

Außerhalb der Betrachtung bleiben weitgehend schulische Ausbildungen mit ihrem sehr hohen Anteil von jungen Frauen, während die »duale Ausbildung« vor allem in ihrem gewerblich-technischen Segment nach wie vor als eine männlich bestimmte Domäne angesehen werden kann. ${ }^{4}$ Unter Chancengesichtspunkten weniger beachtet werden zudem auch die Wege ins Studium. Vor diesem Hintergrund setzt sich eine integrationspolitische Konzentration auf die Öffnung von »dualer Berufsausbildung « möglicherweise dem Verdacht aus, auf ihre Weise dem Modell einer schicht- und herkunftsabhängigen, aber auch genderbezogenen "Passung" von Bildungsgängen zu folgen oder sie sogar noch zu verstärken. Was ist damit gemeint? Durch die Geschichte der deutschen Berufsausbildung hindurch ist gut zu erkennen, dass ihre Funktion vor allem darin bestand, die Kinder - und hier vor allem die Söhne - aus der Arbeiterschaft als Nachwuchs in fachlich versierte ausführende Tätigkeiten einzupassen. Diese »soziale Reproduktion« hat mit der Bildungsexpansion der 6oer und 70er Jahre ihre quasi natürliche

3 Vgl. hierzu u.a. Schultheis, Kathrin/Sell, Stefan: „Die drei Sektoren der beruflichen Bildung - Übergangssystem«, https://www.bpb.de/politik/innenpolitik/arbeitsmarktpolitik/187852/uebergangssystem vom 11.8.2014.

4 Paul-Kohlhoff, Angela: »Berufliche Bildung in Deutschland - ein männliches Projekt?«, in: Kuda, Eva/Strauß, Jürgen/Spöttl, Georg/Kaßebaum, Bernd (Hg.): Akademisierung der Arbeitswelt? Zur Zukunft der beruflichen Bildung, Hamburg 2012, S. 259-266. 
Selbstverständlichkeit verloren, wirkt aber - vor allem vermittelt über schulische Leistungsdifferenzen - stark nach. Trotz aller Reformanstrengungen innerhalb der Berufsausbildung selbst - und auch trotz so fantasiereicher und aufbauender Werbung wie der des Handwerks - ist es nicht gelungen, das Prestige von Berufsausbildung so weit zu erhöhen, dass sie nicht als $» 2$. Wahl« erscheint.

\section{Wie attraktiv ist Berufsausbildung für Jugendliche?}

Verbunden wird mit Berufsausbildung nach wie vor die Erwartung/Befürchtung, beruflich im unteren Teil der sozialen Hierarchie zu landen und wenige Chancen zu haben, daran im Laufe des Arbeitslebens viel ändern zu können. Dem widerspricht auch nicht, dass große und bekannte Betriebe nach wie vor für ihre Ausbildungsplätze eine sehr hohe Anzahl von Bewerber*innen haben. Hier verspricht der Betrieb und nicht der Ausbildungsweg an sich Prestige, gute Bezahlung und Aufstiegschancen.

Man begegnet bei vielen Jugendlichen eine starke Skepsis gegenüber den "Versprechen«, die von der Berufsausbildung ausgehen. Bei Jugendlichen aus Familien mit Migrationsgeschichte sind diese z.T. noch einmal verstärkt durch mangelnde Kenntnis und mangelnden Umgang der Familien mit Berufsausbildung, sowohl im Herkunftsland als auch hier. Oftmals kommt aber unter heutigen Bedingungen auch gymnasiale Bildung und Studium nicht infrage, obwohl der Anteil der Abiturient"innen an allen Schulabgänger"innen stark gestiegen ist. Diese Haltung der Berufsausbildung gegenüber ist auch bei vielen Jugendlichen in den Schulen anzutreffen, die mit Berlin braucht dich! zusammenarbeiten. Schon 2010 wurde erwartet, dass ein wachsender Teil von Jugendlichen mit Migrationsgeschichte den Versuch unternehmen werde, Berufsausbildung zu vermeiden: »Wenig spricht aber für die Annahme, dass sich diese wachsende Gruppe von gut vorgebildeten Jugendlichen mit Migrationshintergrund dann, wenn ihnen der Weg ins Abitur offen steht, anders entscheiden würde/werde als herkunftsdeutsche Jugendliche. $\aleph^{5}$

5 Kruse, Wilfried: Berlin braucht dich! - Über den Öffentlichen Dienst hinaus? Dortmund 2010, S. 5. 


\section{Abitur als die 1. Wahl}

Die damit auch aufgerufene Problematik des »selektiven Bildungssystems« hat Klaus Jürgen Tillmann zusammenfassend so formuliert:

Die enge Verknüpfung von Übergang und Leistungsauslese führt dazu, dass biografische Erfahrungen in einem hohen Maß mit den Kategorien von >Erfolg< und `Versagen` verknüpft sind. Individualisierung bedeutet dann auch, in Laufbahnen unterschiedlichen Prestiges eingewiesen zu werden. Dies alles führt dazu, dass bei solchen Übergängen in besonders starkem Maße ein leistungsorientiertes Selbstbild (sei es positiv oder negativ) geprägt wird. ${ }^{6}$

Berufsausbildung auf Fachkräfteniveau wird - so die damalige Vermutung bei diesen jungen Leuten nur dann wirklich in den Blick kommen, »wenn die Attraktivität von betrieblicher Berufsausbildung als gleichwertiger Option gegenüber Abitur (und Studium) wächst und wenn dies während der mehrjährigen Berufsorientierungsphase auch erfahrbar wird « ${ }^{7}$ Aber wie ist das mit denjenigen, für die das Abitur als erreichbare Option aktuell nicht offensteht? Kann man ihnen raten, sie ermuntern, ihnen nahelegen den Weg in die Berufsausbildung zu gehen - so, wie Berlin braucht dich! es konsortial versucht? Und was ist mit jenen, die dennoch kaum eine Chance auf einen Ausbildungsplatz haben?

Die integrationspolitischen Dilemmata einer Konzentration auf diesen Ansatz sind greifbar, zumal sich die Betriebe, die im Konsortium mitarbeiten, nach wie vor schwertun, ihre Ausbildung vorbehaltslos für genau diese jungen Menschen zu öffnen.

6 Tillmann, Klaus-Jürgen 2013: »Die Bewältigung von Übergängen im Lebenslauf - eine biografische Perspektive«, in: Bellenberg, Gabriele/Forell, Matthias (Hg.): Bildungsübergänge gestalten. Ein Dialog zwischen Wissenschaft und Praxis, Münster 2013, S. 15-32, hier 28.

7 Kruse, Wilfried: Berlin braucht dich! - Über den Öffentlichen Dienst hinaus? Dortmund 2010, S. 5. 


\section{Ein doppelter Ansatz: Betriebe müssen sich für Vielfalt öffnen}

Eine Antwort darauf war und ist ein doppelter, eng miteinander korrespondierender Ansatz, der von Anfang an auch verfolgt wurde, nämlich nicht nur die jungen Leute mit einer Ausbildungsperspektive vertraut $\mathrm{zu}$ machen, sondern die Betriebe - und ihren Lernort-Partner Berufsschule - dazu anzuregen, sich durch eine angemessene Ausbildungsgestaltung auf Vielfalt einzustellen, und zwar einschließlich einer Vielfalt bei den Einstiegsprofilen der Auszubildenden, was schulische Voraussetzungen, bisherige Lernerfahrungen und die Herkunft aus unterschiedlich weit der modernen Arbeitswelt entfernten Milieus betrifft.

Diese Einsicht in die Notwendigkeit eines solchen doppelten, miteinander korrespondierenden Ansatzes war schon früh da, sah sich aber diversen Schwierigkeiten ausgesetzt, die immer wieder die Gefahr einer Vereinseitigung eines nahezu ausschließlichen Blicks auf die Jugendlichen mit sich brachten.

Wie die Wiederaufnahme des doppelten Ansatzes im Rahmen von Berlin braucht dich! und darüber hinaus in Berlin erfolgte, wurde in den vorangegangenen Kapiteln beschrieben. In diesem Zusammenhang markierte das JahresforumExtra »Vielfalt in der Ausbildung«, das gemeinsam von der Arbeitsgemeinschaft Weinheimer Initiative und dem Berliner Integrationsbeauftragten im Dezember 2016 veranstaltet wurde, einen wichtigen Schritt, auch im Hinblick auf die Wiederaufnahme dieses Themas in der Berliner fachlich-politischen Öffentlichkeit. ${ }^{8}$ Auch die 2017 aufgelegte Kampagne Ausbildung-eine Frage der Einstellung mit ihrer Botschaft, dass Betriebe Vielfalt »können«, zielt in dieselbe Richtung.

Dennoch: Die Zugänglichkeit zum »Dualen System der Berufsausbildung« bleibt unter den gegebenen Bedingungen letztlich beschränkt: auf seine »Exklusivität« hat Günter Kutscha auf dem JahresforumExtra 2016 noch einmal nachdrücklich hingewiesen:

Zur Vielfalt gehört auch jener Teil der Jugendlichen, die sich der Eingliederung in das Ausbildungs- und Beschäftigungssystem mangels realistischer Perspektiven oder in Folge von »Selbstausgrenzung« aufgrund subjektiver

8 www.kommunale-koordinierung.de/uploads/tx_news/JFX_2016_Kutscha_Vielfalt_Thesen_Ver18-12-2016.pdf 
Erfahrungen mit der fehlendenden bzw. defizitären Integrationskraft des Übergangssystems entziehen ... Mehrfach haben die Spitzenverbände der Jugendhilfe an einer einseitigen, auf Arbeitsförderung und Eingliederung in das Ausbildungssystem zentrierten Politik der »Bestenauslese« im Rahmen berufsvorbereitender Maßnahmen öffentlich Kritik geäußert und eine stärkere Berücksichtigung der Problemlagen jener Jugendlichen gefordert, für die das Ausbildungs- und Beschäftigungssystem keine Integrationschancen bereit hält. Schutzsuchende und Asylanten sind derzeit davon besonders betroffen ... Bildung im Medium des Berufs? - Ja, wo immer die Lebenssituationen dafür eine sinnvolle Voraussetzung bieten. Allerdings wird sich unsere Cesellschaft dem Problem stellen müssen, allen Jugendlichen in aller Vielfalt auch außerhalb formalisierter Berufslaufbahnen Aufgaben anzubieten und Angebote zu machen, bei denen sie sich bewähren können und ihren Eigensinn unter Beachtung der Regeln gesellschaftlichen Zusammenlebens entfalten können. ${ }^{9}$

\section{Ein erweitertes Verständnis vom Übergang Schule - Arbeitswelt}

Integrationspolitisch kann dies nur bedeuten, von einem erweiterten Verständnis von Übergängen, hier: von Übergängen in die Arbeitswelt, auszugehen und die verschiedenen Übergangswege oder auch Übergangsschicksale insgesamt in den Blick zu nehmen, also danach zu fragen, wie diese sich zu einem Übergangssystem verknüpfen und welche Qualität dieses System hat. Damit ist schon gesagt, dass wir die enge und problematische Einengung der Definition von Übergangssystem als eines "Systems zusätzlicher Maßnahmen« zugunsten eines weiten Verständnisses überschreiten, in dem der Maßnahmen-Komplex als ein Teil dem gesamten bestehenden Übergangssystem zugerechnet wird.

Zum erweiterten Verständnis von Übergängen in die Arbeitswelt wird auf eine Argumentation zurückgegriffen, die anlässlich der Abschlussveranstaltung des Berliner Projekts »Regionales Übergangsmanagement« (RÜM) 2012 entwickelt wurde: ${ }^{10}$

\footnotetext{
9 www.kommunale-koordinierung.de/uploads/tx_news/JFX_2016_Kutscha_Vielfalt_The sen_Ver18-12-2016.pdf

10 Vgl. Kapitel 3.
} 
Was also ist Sache? Wir blicken auf mehr als ein Jahrzehnt einer Ausbildungsmarktkrise zurück, mit tiefgreifenden Folgen für mehrere Jugendlichen Generationen, von denen ein nicht unerheblicher Teil große Schwierigkeiten hatte, erfolgreich in Ausbildung und Arbeitsleben einzumünden oder sogar scheiterte. Das hat tiefe Spuren im Bewusstsein hinterlassen: das Vertrauen in die »Königswege« in die Arbeitswelt, das es einst gab, ist dahin. Und zwar wohl mit Recht: denn an Stelle der einstmaligen Verlässlichkeit von Königswegen ist der Zwang getreten, sich seinen Weg zu suchen und zu finden und diesen Weg zu optimieren. Jene fest umrissenen Wege, von denen in der Vergangenheit ausgegangen wurde, nämlich der direkte Durchstieg für Kinder aus den bürgerlichen Familien über Abitur in eine akademische oder Dienstleistungs- oder Verwaltungs-Karriere oder für die Kinder aus der Arbeiterschaft mit einer Ausbildung auf einen Arbeitsplatz, wo man ein Leben lang bleibt, sind nicht mehr die Regel, sondern die Ausnahme. Es geht demzufolge darum, darauf zu achten, dass alle Wege in die Arbeitswelt hinein für die Heranwachsenden ein Optimum an Chancen für die Lebensgestaltung beinhalten.

Schwierigkeiten, einen guten Weg von der Schule in die Arbeitswelt zu finden, haben heute nicht nur diejenigen, die wir gemeinhin als die Benachteiligte bezeichnen. Sondern: generelle Orientierungsschwierigkeiten im Übergang von der der Schule in die Arbeitswelt und auch die Suchbewegungen, die damit verbunden sind, haben sich im Grunde genommen nahezu auf alle Heranwachsenden ausgedehnt. Der Übergang Schule - Arbeitswelt ist für Jugendlichen insgesamt zu einer erheblichen Herausforderung geworden, wenngleich nicht für alle gleichermaßen risikoreich. Wenn man über dann über Handlungsprioritäten redet, muss man auf diejenigen achten, bei denen diese Orientierungsprobleme zu einem besonderen Risiko werden. ${ }^{11}$

11 Kruse, Wilfried: »Dimensionen landesweiter Steuerung am Übergang Schule - Beruf«. Vortrag anlässlich der Abschlussveranstaltung des BMBF-Projektes RÜM Berlin am 15.03.2012 im Palais Kulturbrauerei, Berlin. www.perspektive-berufsabschluss.de > downloads und: vimeo.com > XENOS Panorama Berlin > Videos 


\section{Übergangsgestaltung als Daueraufgabe}

Warum also ist davon auszugehen, dass es im Übergang von der Schule in die Arbeitswelt dauerhaft Herausforderungen und Probleme geben wird, und nicht nur konjunkturell oder zwischenzeitlich? Das hat im Wesentlichen fünf Gründe.

Erstens: Schule und Arbeitswelt sind zwei ganz unterschiedliche gesellschaftliche Teilsysteme, die eigene Logiken und Entwicklungsdynamiken haben. Dass z.B. die Arbeitswelt Dynamiken von Anforderungsveränderungen und Turbulenzen kennt, haben wir in den letzten Jahren verstärkt erfahren können, während das Schulsystem bei allen Veränderungen in den letzten Jahren insgesamt gesetzter, verregelter und schwerfälliger geworden ist. Von daher gibt es hier immer eine Art Grundspannung und es ist nicht davon auszugehen, dass man die beiden Teilsysteme passgenau aufeinander beziehen kann. Wer glaubt, das zu können, der folgt einer planwirtschaftlichen Illusion, die für unsere komplexen Gesellschaften nicht mehr tragfähig ist. Von daher wird es am Übergang Schule - Arbeitswelt immer zu Schwierigkeiten kommen. Die Frage ist: Wie groß werden die Schwierigkeiten? Wie stark betreffen sie die einzelnen?

Zweitens: Wenn man den direkten Weg der dualen Berufsausbildung für einen wichtigen, möglicherweise für den wichtigsten Weg neben dem $\mathrm{Ab}$ itur/Studium hält, dann muss man immer berücksichtigen, dass die duale Berufsausbildung von ihren wesentlichen Charakteristika her vor allem Teil des Beschäftigungssystems ist und an einzelbetrieblichen Entscheidungen hängt, und dies vor allem in der Frage, wie viele betriebliche Ausbildungsplätze angeboten werden und in welchen Berufen ausgebildet wird. Aber vor allem auch: Wer wird als Auszubildende*r eingestellt? Dieser Einstellungsvorbehalt ist ein wesentliches Charakteristikum unseres dualen Ausbildungssystems. Von daher ist nicht zu erwarten, dass es einen reibungslosen Ausgleich zwischen denen gibt, die einen Ausbildungsplatz haben wollen, und denen, die Ausbildungsplätze bieten, weil der Markt dazwischen ist.

Drittens: Die »Königswege« haben sich aufgelöst. Die Jugendlichen und jungen Erwachsenen von heute haben es offenbar subjektiv viel schwerer, für sich den richtigen Weg zu finden, als in der Vergangenheit. Zugleich stellt sich für sie auch die Sinnfrage stärker als früher: Was mache ich mit meinem Leben? 
Viertens: Die Phase von Bildung und Ausbildung hat sich biographisch verlängert, wenn man als ihren Endpunkt eine Situation annimmt, in dem eine Person durch Arbeit ein eigenständiges Leben führen kann. Der alte Spruch »Erst die Ausbildung, dann das Leben« greift nicht mehr. Die wichtigen Lebensaufgaben reihen sich nicht mehr aneinander, sondern stellen sich gleichzeitig, was die persönlichen Lagen insgesamt wesentlich krisenanfälliger macht.

Fünftes: Die veränderten Vorzeichen auf dem Arbeitsmarkt und der im Hintergrund drohende Fachkräftemangel sorgen zwar für Entspannung auf der Angebotsseite des Ausbildungsgeschehens, aber sie erledigen für bestimmte Gruppen von Jugendlichen die Schwierigkeit, einen Ausbildungsplatz zu finden, nicht. Es müssen Unterstützungssysteme etabliert werden, die es Jugendlichen mit schwierigeren Einstiegsvoraussetzungen erleichtern, die betrieblichen Anforderungen sowie die Anforderungen der Berufsschule zu bestehen. Ausbildungssicherung, die sich sowohl an die Auszubildenden als auch an die ausbildenden Betriebe richtet, wird ein zentrales Element sein wird, wenn man das Ankommen in der Arbeitswelt stärken und stützen will.

In diesem fünffachen Sinne also bleibt Übergangsgestaltung Daueraufgabe. Das heißt, die systematische Einflussnahme darauf, dass den jungen Erwachsenen erfolgreiche Einstiege in die Arbeitswelt gelingen, bleibt eine zentrale dauerhafte und damit auch öffentliche Aufgabe. Dies kann die öffentliche Hand allerdings nicht allein, sondern nur in Kooperation bewältigen. Oder noch einmal zusammenfassend formuliert: Übergangsgestaltung darf nicht an oder vor der ersten Schwelle stehen bleiben. Gemeint ist damit der Übergang von der Schule in eine Ausbildung oder ein Studium. Sie muss die lang gestreckten übergangsbiografischen Verläufe ins Auge fassen. (Stichworte u.a.: Sicherung von Ausbildungserfolg und Einstieg in Arbeit nach Ausbildungsende). Das »Lehrstück Übergang« zwingt dazu, nicht nur Wirtschaft und Arbeitsmarkt in Betracht zu ziehen, sondern auch die Sicht der Jugendlichen und jungen Erwachsenen selbst. Attraktivität und Qualität von Ausbildung sowie Fragen von Zugang, Gleichwertigkeit und Durchlässigkeit werden so - aus beiden Perspektiven - zu zentralen Zielgrößen. 


\section{Prämissen für ein förderliches Übergangssystem}

Aus diesen Zusammenhängen lassen sich wichtige »Bauprinzipien« für ein Übergangssystem entwickeln:

Gelingender Übergang ist eine wichtige biografische Weichenstellung: Alle jungen Menschen, die in Berlin aufwachsen, benötigen Bildung und Ausbildung, die ihnen persönliche Entwicklungsoptionen bieten, Grundlage für eine selbständige Lebensführung sind und die gesellschaftliche Teilhabe fördern. Beachtet werden muss dabei, dass Berlin eine Metropole ist, die durch Einwanderung wesentlich mitgeprägt ist. Gelingende Übergänge von der Schule in die Arbeitswelt sind für alle als biografische Weichenstellung von großer Bedeutung.

Gelingende Übergänge sind wichtig für die soziale, wirtschaftliche und demokratische Zukunft Berlins: Berlin braucht diese jungen Menschen für eine soziale, demokratische, wirtschaftlich starke und lebenswerte Zukunft in der Metropole. Gute Bildung und Ausbildung für alle sind der Motor der künftigen Entwicklung Berlins und sichert Kontinuität und Innovation. Dies gilt insbesondere auch für Berlin als modernem Industriestandort und in Hinblick auf eine soziale Infrastruktur, wie sie alle Generationen für ein gutes und sicheres Leben benötigen. Gelingende Übergänge sind deshalb als $\mathrm{Zu}$ kunftssicherung für Berlin von großer Bedeutung.

Neues konsistentes und optionsreiches Übergangssystem: Das Land Berlin benötigt deshalb ein Übergangssystem »aus einem Wurf, dessen Aufgabe es ist, gelingende Übergänge von der Schule in die Arbeitswelt für alle Jugendlichen zu fördern und zu sichern. Dabei geht es keineswegs darum, »das Rad neu zu erfinden«, sondern an Bestehendem und Bewährtem anzuknüpfen, aber auch neue Bausteine hinzuzufügen, wo dies erforderlich ist. Insbesondere sollen aber Verknüpfungen in einer Weise hergestellt werden, dass im Ergebnis das neue Übergangssystem in sich konsistent und transparent ist, seine Wege für die Jugendlichen optionsreich sind und seine laufende Überprüfung und Weiterentwicklung erfolgt.

Erweitertes Verständnis von Übergang: Es wird ein in zweifacher Weise erweitertes Verständnis von Übergang und Übergangsgestaltung zugrunde gelegt. Zum einen wird ein längerer Zeitraum ins Auge gefasst, der nicht schon bei der »1. Schwelle«, sondern erst dann endet, wenn der junge Erwachsene in der Arbeitswelt gut angekommen ist. Dieser Zeitraum umschließt also ausdrücklich auch die Phase der Berufsausbildung. Zum anderen richtet 
sich das »Neue Übergangssystem «nicht nur an Benachteiligte, sondern an alle, weil für alle die Übergänge im Sinne des Wortes problematisch geworden sind. Vermeidung, Verringerung und Korrektur von Benachteiligungen bleiben aber zentrale Gestaltungsaufgaben.

Verantwortung und Verantwortungsgemeinschaft: Es ist öffentliche Verantwortung, für eine wirkungsvolle Gestaltung des »neuen Übergangssystems « Rechnung zu tragen. Dies kann die öffentliche Hand aber ohne eine enge Zusammenarbeit mit allen Akteuren, die im Übergang verantwortlich tätig sind, nicht leisten. Erfolgreiche Übergangsgestaltung setzt eine breite, verbindliche und handlungsfähige Verantwortungsgemeinschaft voraus.

Bildungstyp Berufsausbildung muss aufgewertet werden: Dem erweiterten Verständnis von Übergang folgend, macht es Sinn, vom Zeitpunkt her zu blicken, an dem das Ankommen in der Arbeitswelt gut gelungen ist. Aus dieser Sicht bedarf derjenige Bildungsweg, der neben Abitur und Studium in die Arbeitswelt führt, nämlich die Berufsausbildung, einer Aufwertung. Hier stehen zwei miteinander korrespondierende Ansätze im Vordergrund: erstens die Mobilisierung und Sicherung weiterer qualifizierter betrieblicher Ausbildungsplätze, und zweitens die Verbesserung der Attraktivität der Ausbildung für Jugendliche, insbesondere auch für jene, die bislang zur betrieblichen Berufsausbildung keinen Zugang gefunden haben oder die Option nicht interessant fanden. Eine aktive betriebliche Haltung der »Öffnung« und der »Willkommenskultur« ist hierfür unverzichtbar.

Knappes Gut Lernort Betrieb mobilisieren: Bei der Ausweitung des Angebots an betrieblichen Ausbildungsplätzen steht stets ihre Qualität im Vordergrund, und zwar sowohl in fachlicher als auch in pädagogischer Hinsicht. Hierzu werden Zielgrößen entwickelt. Der Ausbau überbetrieblicher Ausbildungsverbünde, die Antwort auf die Berliner Betriebsstruktur sind, ist eine wichtige Option. Auch die Tarifvertragsparteien können sich die Mobilisierung weiterer Ausbildungsplätze guter Qualität zur Aufgabe machen. Die dynamische Entwicklung, die das »duale Lernen« in seinen verschiedenen Formen findet, demonstriert, dass der Lernort Betrieb im Zuge des Übergangs von der Schule in die Arbeitswelt unverzichtbar ist. Die Sicherung des Lernorts Betrieb in ausreichender Menge und guter Qualität ist deshalb eine zentrale Aufgabe der »Berliner Verantwortungsgemeinschaft Übergang«.

Das berufsqualifizierende System neu justieren: Stabilisierung, Ausbau und Weiterentwicklung der betrieblichen Berufsausbildung (sogenanntes »duales System«) sind zentral; die betriebliche Berufsausbildung ist und 
bleibt aber nur ein - wenn auch sehr wichtiger - Teil des Berliner Systems der Berufsbildung. Die vollschulischen Ausbildungsgänge haben ebenfalls ein erhebliches Gewicht. Ein weitere »Säule« umfasst diverse ausbildungsvorbereitende oder teilqualifizierende Maßnahmen, die eine "Brücke« in Ausbildung bilden sollen. Dieses in sich differenzierte Berliner berufsqualifizierende System wird auf Konsistenz, Qualität und Optionshaltigkeit jedes Ausbildungsgangs hin erneut überprüft und sortiert. Als Prinzipien hierbei müssen gelten: Anschlussfähigkeit, Gleichwertigkeit und Förderung von Diversität. Die Oberstufenzentren bilden berufsschulisch die »Klammer« zwischen allen Teilen des berufsqualifizierenden Bildungssystems. Sie müssen bei der Neujustierung als Berufliche Kompetenzzentren in den Blick genommen werden.

Entwicklungspotenziale der Jugendlichen ins Zentrum rücken: Weder die Vorstellung, dass manchen Jugendlichen lediglich noch die Disziplin betrieblicher Arbeit helfen könne, noch die Idee von der "Passgenauigkeit» sind vor diesem Hintergrund wirklich tragfähig. Auch die die Formel, dass »eder Ausbildungsplatz besser als keiner« sei oder der unhinterfragte Vorrang »dualer« vor jeder anderen Form der Berufsbildung ist in dieser Allgemeinheit zweifelhaft. Zentraler Bezugspunkt müssen die Interessen und Fähigkeiten und die eigenen Lebensentwürfe der Jugendlichen und jungen Erwachsenen bleiben. Heute sind wir von der Gleichwertigkeit der verschiedenen Wege ins Arbeitsleben noch weit entfernt. Sie wäre besser gewährleistet, wenn die Zugangschancen zu den verschiedenen Wegen in die Arbeitsund Erwachsenenwelt sozial weniger selektiv wären, ihre jeweilige Qualität die Optionsmöglichkeiten der Lernenden erweitern würden und wenn sich einmal getroffene Wegeentscheidungen mit vertretbarem Aufwand korrigieren ließen.

Koordinierungssystem etablieren; "Fokus Migration « systematisch berücksichtigen: Allerdings braucht das »Neue Übergangssystem« auch dann, wenn es etabliert wird, eine dauerhafte Koordinierung. Dies ist in dem stetigen Optimierungsbedarf, der durch eine zyklische Arbeitsweise von regelmäBigen Bilanzierungen und der Verabredung zu zeitlich gefristeten Handlungsplänen hinterlegt wird, begründet. Das System muss korrektur- und innovationsfähig bleiben und auch die Mobilisierung des Lernorts Betrieb ist eine kontinuierliche Herausforderung. Koordinierung ist sinnvoller Weise als »Zwei-Ebenen-Ansatz« zu praktizieren: nämlich als landesweite Koordinierung und als »Koordinierung vor Ort« auf Bezirksebene, weil sie dort 
lebensweltnah angesiedelt ist. Mechanismen, die diese beiden Ebenen in Korrespondenz zueinander setzen, müssen entwickelt werden.

\section{DGB: Gelingende Übergänge von der Schule in die Arbeitswelt}

Die Frage, wie Übergänge von der Schule in die Arbeitswelt für alle besser gelingen können als in der Vergangenheit, ist immer wieder Thema des öffentlich-politischen Diskurses. In diesen Diskurs hat sich der DGB BerlinBrandenburg mehrfach pointiert eingeschaltet.

Der DGB Landesbezirk Berlin-Brandenburg hat auf zwei aufeinander folgenden Konferenzen, nämlich 2014 und 2018, grundlegende Beschlüsse zur Notwendigkeit eines Berliner Übergangssystems gefasst. ${ }^{12}$

Unter der Überschrift »Gelingende Übergänge von der Schule in die Arbeitswelt: Zukunftssicherung für die Metropole Berlin« heißt es u.a.: "Deswegen muss besonderes Augenmerk auf die Qualität der Berufsausbildung selbst gerichtet werden, insbesondere auch in Hinblick auf die gewachsenen pädagogischen Herausforderungen. Dabei sind beide Lernorte der dualen Berufsausbildung von Bedeutung, also nicht nur die Betriebe, sondern auch die Berufsschulen und insbesondere ihre pädagogische Kooperation. Die Mobilisierung des Lernorts Betrieb ist eine zentrale $\mathrm{Zu}$ kunftsaufgabe ${ }^{13}$ Dies wird als eine gemeinschaftliche Aufgabe aller Akteure verstanden und in dieser Hinsicht positiv auf den Konsortialansatz von Berlin braucht dich! bezuggenommen. Berlin braucht dich!, die Einführung des WAT-Unterrichts und des »dualen Lernens", die Vorlage des Landeskonzepts der Berliner Berufs- und Studienorientierung und das Modell der Berliner Jugendberufsagenturen mit ihren bezirklichen Standorten werden als neue Strukturelemente im Übergangssystem genannt; aus Sicht des DGB stehen sie aber additiv nebeneinander und nicht »kohärent« aufeinander bezogen. Erforderlich sei: »Die unterschiedlichen Modelle und Koordinierungsansät-

12 IC Metall Berlin: Beschluss Bo02: Gelingende Übergänge von der Schule in die Arbeitswelt: Zukunftssicherung für die Metropole Berlin. In: DGB Berlin-Brandenburg: Beschlüsse der 7. DGB Bezirkskonferenz Berlin-Brandenburg 2018.

13 Ebd. 
ze sind weiterzuentwickeln $\mathrm{zu}$ einem landesweit abgestimmten Landesprogramm >Neues Übergangssystem Berlin«.«

\section{Übergänge: ein Lehrstück}

Schließlich ist die Frage einer guten Übergangsgestaltung auch eng damit verknüpft, wie sich Zusammenleben und Demokratie künftig entwickeln werden; Übergang ist also auch ein Lehrstück darüber, welche Teilhabechancen die Gesellschaft bietet. Dieser Zusammenhang wurde schon bei einer der ersten Jahresforen der Arbeitsgemeinschaft Weinheimer Initiative 2011 aufgerufen:

Die Erfahrungen, die die jungen Erwachsenen im Übergang machen, sind von daher zugleich und ganz wesentlich auch als Erfahrungen mit unserer Cesellschaft und ihren Umgang mit zentralen Werten zu verstehen. Der Umgang mit den jungen Menschen im Übergang muss deshalb von Anbeginn an und durchgehend durch Respekt vor ihrer eigenständigen Persönlichkeit und durch Solidarität geprägt sein. Respekt und Wertschätzung übersetzen sich im Übergangsgeschehen vor allem darin, ein realistisches Bild des Arbeitslebens und der in ihm vorhandenen Perspektiven erfahrbar zu machen und zu vermitteln und den jungen Leuten deren eigene Startbedingungen und Voraussetzungen und deren Optimierungsmöglichkeiten kritisch zu spiegeln, ohne sie zu bedrängen, zu nötigen oder in Panik zu versetzen. Sich Ausprobieren schließt ein, dass Entscheidungen auch ohne unangemessene persönliche Kosten revidierbar sein müssen. ${ }^{14}$

\section{Fazit}

Im Kapitel 10 wurde ein Bogen zur Ausgangssituation um das Jahr 2007 geschlagen, die vor allem Thema des ersten Kapitels war. Nachgezeichnet wurde dort für das Feld des Übergangs von der Schule in die Arbeitswelt

14 Arbeitsgemeinschaft Weinheimer Initiative: Hoyerswerdaer Erklärung - Lehrstück Übergang. Jahresforum 2011, in: Arbeitsgemeinschaft Weinheimer Initiative (Hg.): Lokale Bildungsverantwortung, Stuttgart 2013, S. 398-401. 
eine integrationspolitisch grundierte doppelte Konzentration: auf die duale Berufsausbildung und zunächst auf den Öffentlichen Dienst, mit späteren Ausweitungen auf die Betriebe mit Landesbeteiligung und die Metall- und Elektro-Branche.

Die integrationspolitische Konzentration auf die duale Berufsausbildung ist auf der einen Seite durch das Entwicklungspotenzial, das Bildung im Medium von Betrieb und Beruf vorhält, wie in Kapitel 7 ausgeführt, gut begründet. Auf der anderen Seite ist die duale Berufsausbildung nur ein Segment oder ein Weg im Übergang von der Schule in die Arbeitswelt, der zudem gegenüber weiterführender Schule und Abitur weniger attraktiv erscheint, zugleich aber aufgrund der entscheidenden Rolle, die die ausbildenden Betriebe spielen, auch mit beträchtlichen Zugangsbarrieren verbunden ist, wie im Kapitel 8 am Beispiel der Schüler"innen aus den bei Berlin braucht dich! mitarbeitenden Schulen beispielhaft gezeigt wurde. Im doppelten Ansatz weiter verbesserter, den Lernort Betrieb nutzender, aber schulisch gut gestalteter Berufsorientierung und einer vielfaltorientierten hohen Ausbildungsqualität in Betrieb und Berufsschule in Kooperation zwischen ihnen wurde eine Option gesehen, erhöhte Attraktivität mit niedrigschwelliger $\mathrm{Zu}$ gänglichkeit zu dualer Ausbildung zu verbinden.

Es ist aber - so wird im vorliegenden Kapitel 10 argumentiert - auch integrations- und teilhabepolitisch geboten, gegenüber dieser Engführung auf »duale Ausbildung« ein erweitertes Verständnis des Übergangs von der Schule in die Arbeitswelt zugrunde zu legen. Dieses erweiterte Verständnis bezieht sich nicht nur auf die verschiedenen Wege, die für den Übergang bereitstehen, sondern auch darauf, den Übergang als einen biografisch langgestreckten Prozess zu verstehen, der im Grunde erst endet, wenn die jungen Erwachsenen gut in einer Arbeitstätigkeit angekommen sind.

Die integrations- und teilhabepolitische Fokusfunktion - wie sie in Kapitel 3 skizziert wurde - müsste sich demnach mitgestaltend auf alle Übergangsverläufe beziehen und dazu beitragen, aus ihnen ein transparentes Übergangssystem zu formen, in dem ein Übergang zwischen verschiedenen Wegen und deren Kombination niedrigschwellig möglich wird. Hierzu werden einige Prämissen formuliert, die dazu dienen könnten, die Öffnung von Berufsausbildung chancenreich einzubetten.

Schon in den 2o10er Jahren wurde, wie in Kapitel 3 skizziert, die Formung eines Berliner Übergangssystems hin und wieder zum Thema, zumal dies in anderen Bundesländern, wie z.B. in Hamburg und in NRW, tatsäch- 
lich in Angriff genommen wurde. Im Zeitraum seit 2007, als das Integrationskonzept vorgelegt wurde, bis heute sind in vielen Teilfeldern wichtige Ansätze und Modelle entwickelt, Verfahren geklärt und eine Fülle von Erfahrungen und Erkenntnissen gesammelt worden. Der Herausforderungen, die mit dem Ankommen vieler junger Geflüchteter verbunden waren, haben Impulse für weiterführende und vor allem auch strukturbildende Aktivitäten ausgelöst, wie im Kapitel 9 skizziert wurde. Das Erfordernis, Übergänge stets auch unter einer integrations- und teilhabeorientierten Perspektive zu betrachten und zu gestalten, ist in dieser Zeit nicht nur deutlicher geworden, sondern findet auch im Vergleich zu 2007 ungleich mehr Akzeptanz und Zustimmung. Es scheint also ein guter Zeitpunkt zu sein, die Arbeit an einem kohärenten, chancengerechten, vielfaltorientiertem und förderlichen Berliner Übergangssystem auf die Tagesordnung zu setzen.

Dies ist zugleich mit der Frage verknüpft, wie sich die Verhältnisse in unserem demokratischen Gemeinwesen weiter entwickeln werden. Denn für die jungen Menschen, die hier aufwachsen, ist der Übergang Schule Arbeitswelt nicht nur eine wichtige Weichenstellung für ihr Leben, sondern zugleich auch ein Lehrstück: Die Erfahrungen, die im Übergang gemacht werden, sind zugleich auch Erfahrungen mit unserer Gesellschaft, ihren Versprechen und ihrem tatsächlichen Umgang mit zentralen Werten. 


\section{Nachbetrachtung}

\section{Andreas Germershausen im Gespräch mit Wilfried Kruse}

Wilfried Kruse: Im ersten Kapitel wird das Integrationskonzept von 2007 als Beginn einer neuen Periode des Umgangs mit Einwanderung in den Blick gerückt. Kann man diese Einschätzung auch in der Rückschau noch aufrechterhalten?

Andreas Germershausen: Sicherlich bietet das Konzept von 2007 einen grundsätzlich neuen Ansatz, und zwar in zweierlei Hinsicht: Erstens schafft es die Grundlage für eine auf Partizipation zielende Integrationspolitik der Landesregierung insgesamt. Partizipation und Integration sind als Querschnittsansatz in allen Handlungsfeldern der Senatspolitik umzusetzen.

Zweitens werden für die Handlungsfelder, die federführend von den einzelnen Senatsverwaltungen umzusetzen sind, Indikatoren definiert, anhand derer eine Erfolgsmessung erfolgen kann. Diese Indikatoren bieten die Grundlage für ein Monitoring, das in der Folgezeit alle zwei Jahre durchgeführt wurde; zunächst als Berliner Bericht, sodann auf Initiative von Berlin mit NRW als Integrationsmonitoring der Bundesländer. Dieses Berichtswesen ist kein Selbstzweck. Es zeigt an, in welchen Handlungsfeldern sich Verbesserungen oder auch besorgniserregende Entwicklungen abzeichnen - und auch, welche Besonderheiten die Lage in Berlin und auch in anderen Bundesländern charakterisieren.

Mit dem Monitoring ist der Senat weg von einer reinen Inputorientierung zu einer Ergebnisorientierung gegangen. Die Politik soll sich an ihrem Output messen lassen, und auf Basis des Ergebnisses wird die Politik ggf. 
umsteuern. Dieser Ansatz schuf eine solide Strukturierung für das nächste Jahrzehnt.

Das dritte Element, das 2007 sehr wichtig war, nämlich so genannte Leitprojekte der einzelnen Verwaltungen vorzustellen, hat sich langfristig als weniger stark erwiesen. Sicherlich gibt es immer wieder besonders wichtige Vorhaben. Diese werden aber mittlerweile weniger stark mit einer strukturierenden Integrationspolitik verbunden.

W.K.: Wie erklärt sich, dass beim damaligen Integrationskonzept die Verknüpfungen zwischen den strategischen Feldern, wie Integration ins Erwerbsleben und Integration in Bildung, schwach blieben?

A.G.: Das hat zwei Gründe: Erstens ist der Integrationsbeauftragte nicht in dem Maße als Koordinierungsstelle gestärkt worden, dass er den Querschnittsansatz gegenüber anderen Fachverwaltung nachhaltig durchsetzen konnte. Zweitens beobachte ich in der Integrationspolitik in unterschiedlichen Phasen die Betonung unterschiedlicher Themen. So stand Anfang der $2000 e r$ Jahre die Bildungspolitik unter Druck, da mit dem PISA-Schock deutlich wurde, wie eng der Bildungserfolg an den Bildungserfolg der Eltern und unterschiedliche Milieus gebunden war - mit im Durchschnitt negativem Ergebnis für Heranwachsende aus Einwandererfamilien. Mit dem $\mathrm{Zu}$ wanderungsgesetz von 2005 rückte der Fokus auf den Spracherwerb. Erst später, etwa ab 2008, gewinnt die Frage der Qualifizierung und des Fachkräftebedarfs an Bedeutung. Diese bleibt - aufgrund der für einen breiteren Kreis bedeutsam werdenden demographischen Entwicklung - wichtig. Gleichwohl habe ich - nach der bis März 2016 starken Flüchtlings-Zuwanderung - mitunter den Eindruck, als hänge der integrationspolitische Erfolg vom Wohnungsbau ab.

Natürlich setzen Partizipation und Integration Erfolg in allen Handlungsfeldern voraus: in Bildung, Sprache und beruflicher Kompetenz ebenso wie beim eigenständigen Zugang zu Wohnraum. Zudem übersehe ich nicht, dass in den kurz skizzierten Phasen in den einzelnen Handlungsfeldern Erfolge erreicht wurden. Die Fixierung auf einzelne Themen hat jedoch die Beachtung von in der Frage genannten Querverbindungen und die gleichzeitige Umsetzung des Querschnittsansatzes in allen Handlungsfeldern eingeschränkt. 
W.K.: Im Feld »Integration ins Erwerbsleben« wurde die Öffnung der Ausbildung im Öffentlichen Dienst priorisiert. Hätte es hierzu Alternativen gegeben? Wie sind die Folgen dieser Entscheidung aus heutiger Sicht zu beurteilen?

A.G.: Mit der Orientierung auf den Öffentlichen Dienst hat der Senat als Arbeitgeber Verantwortung zeigen wollen und gezeigt. Es gab bei der damaligen Integrationssenatorin Dr. Knake-Werner und dem Innensenator Dr. Körting den gemeinsamen Wunsch, den Öffentlichen Dienst bunter zu gestalten. Angesichts eines mehrere Jahre lang recht rigide durchgesetzten Einstellungstopps wich man auf die Ausbildung aus. Zwar ergab sich daraus sofort die kritische Frage, warum man für ein Segment ausbilde, das nicht einstellt. Mittelfristig zeitigte jedoch der Schwerpunkt auf die Ausbildung im Öffentlichen Dienst herausragende Ergebnisse. Dass der Senat als Arbeitgeber signalisierte »Berlin braucht dich!«, war eine großartige Botschaft in die Einwanderungs-Communities.

In der Folge gelang die Ausweitung auf andere Branchen, namentlich auf die Metall- und Elektroindustrie und auf die Unternehmen, an denen das Land beteiligt ist (Öffentliche Unternehmen), die in vielen Gewerken ausbilden. Sofern Du meinst, dass die Berufsausbildung nicht insgesamt interkulturell geöffnet werden konnte, liegt das m.E. nicht an der Fokussierung zunächst auf den Öffentlichen Dienst.

W.K.: Das Partizipations- und Integrationsgesetz von 2010 (PartIntG) rückt die Figur des Integrationsbeauftragten ins Zentrum. De facto aber wird seine strukturelle Handlungsfähigkeit durch die Einordnung in eine Senatsverwaltung und durch Mängel in der Ausstattung eingeschränkt. Wie erklären sich aus heutiger Sicht diese damaligen Schieflagen? Hätte es Alternativen gegeben?

A.G.: Während der Entwicklung des Gesetzes wurden Optionen erörtert, wie man eine stärkere Unabhängigkeit des/der Beauftragten erreichen könnte. Vorgeschlagen wurden die Zuordnung zur Senatskanzlei und damit die Anbindung an den Regierenden Bürgermeister oder die Verortung im Landesparlament; beides wurde aus nachvollziehbaren Gründen verworfen.

Die Einordnung in eine Senatsverwaltung wurde nicht erst durch das PartIntG geschaffen. Auch zuvor schon war der Integrationsbeauftragte 
einer Senatorin und administrativ einer Staatssekretärin zugeordnet. Die Festlegung der Kompetenzen des oder der Integrationsbeauftragten im Gesetz bewirkte jedoch Folgerungen, die zum Teil nicht intendiert waren. Eindeutig positiv ist, dass das eigene Presserecht und die die Ombudsfunktion des Beauftragten festgeschrieben wurden. Letztere bedeutet, einzelnen Migrant*innen oder Gruppen eine Stimme zu geben und ggf. ihre Interessen mit zu vertreten.

Einschränkend hat sich die Betonung des Ressortprinzips ausgewirkt. Dabei ist der Bezug auf dieses Prinzip nicht zu kritisieren, setzt sich der Senat doch aus Senatorinnen und Senatoren zusammen, die jeweils ihre Ressorts leiten. In der Folge dieses Prinzips werden die Handlungsmöglichkeiten des/der Integrationsbeauftragten im PartIntG (\$ 5) durchgängig als »im Auftrag der für Integration zuständigen Senatsverwaltung« oder »des für Integration zuständigen Senatsmitglieds« definiert. Das wirkt so, als würde die Zuständigkeit der/des Beauftragten, die/der Beauftragte/r des Senats ist, auf das Aufgabenfeld der Senatsverwaltung für Integration eingeschränkt, obgleich das tatsächlich nicht der Fall ist.

Eine objektive Schwäche des Gesetzes liegt darin, dass die/der Integrationsbeauftragte keine wirksamen Instrumente zur Durchsetzung des Gesetzes erhalten hat. Das geht auf die Lage während der Erarbeitung des Gesetzes zurück. Der Senat hätte das Gesetz 2010 nicht beschlossen, wenn damit finanzielle Auswirkungen verbunden gewesen wären. Nachfolgende Senate haben das nicht korrigiert.

W.K.: Bedarf das Partizipations- und Integrationsgesetz nach den seit 2010 gemachten Erfahrungen einer Novellierung?

A.G.: Eine Novellierung halte ich für dringlich. Sie sollte die erwähnten Schwächen korrigieren. Ich habe 2018 eine Evaluation in Auftrag gegeben, die Empfehlungen zur Weiterentwicklung des Gesetzes erarbeitet hat. Welche Vorschläge man umsetzen kann, wird im Novellierungsverfahren entschieden, das nach derzeitigem Plan noch in der laufenden Legislaturperiode (bis September 2021) abgeschlossen werden soll.

Durch die Gesetzesnovelle sollte die Kompetenz des/der Beauftragten im Hinblick auf ressortübergreifendes Handeln gestärkt werden. Man sollte sich dabei am Modell des Gender Mainstreaming orientieren und ein Interkultur- oder Migrations-Mainstreaming entwickeln. Zu dessen Umsetzung 
sollte die/der Beauftragte das Recht erhalten, direkt mit den Senatorinnen und Senatoren der relevanten Fachverwaltungen in Verhandlung treten zu dürfen. So kann das »im Auftrag Handeln« eingeschränkt und die Querschnittskompetenz für Partizipation und Integration gestärkt werden.

Die anderen erwähnten Organisationsvorschläge würde ich hingegen nicht erneut aufgreifen. Gegen eine Anbindung beim Regierenden Bürgermeister spricht, dass Differenzen um Integrations- und Migrationspolitik dann stets den Senat insgesamt belasten könnten; bei einer Anbindung ans Parlament wäre die Abteilung Integration zu stark von den tatsächlichen Senatsprozessen abgekoppelt.

W.K.: Die beginnenden 2010er Jahre zeigen insbesondere hinsichtlich der Vorbereitung auf die Arbeitswelt unter dem Stichpunkt »Berufsorientierung" einen bildungspolitischen Aufbruch. Hieran hat das aus integrationspolitischer Perspektive betriebene Leitprojekt Berlin braucht dich! einen deutlichen Anteil. Wie kam es zu dieser Impulswirkung?

A.G.: Ich begrüße sehr, dass die Berufsorientierung insgesamt und speziell die Berufsorientierung in der Migrationsgesellschaft in Berlin an Bedeutung gewonnen hat. In die so genannte vertiefte Berufsorientierung sind erhebliche Ressourcen geflossen, und die Landeskonzeption "Berufs- und Studienorientierung« setzt einen deutlichen Akzent auf Schülerinnen und Schüler aus Einwandererfamilien. Das von Berlin braucht dich! entwickelte Konzept der Vierstufigkeit - eine sinnvolle Folge von vier Betriebsbegegnungen in den Schuljahren 7-10 - wurde in die Landeskonzeption »Berufs- und Studienorientierung « aufgenommen.

Dass Berlin braucht dich! eine starke Rolle spielen konnte, lag an dem schon zu Beginn im Jahr 2006 erkennbaren Erfolg des Ansatzes. Man hatte eine so lange Geschichte von Berichten über Benachteiligung hinter sich, über den hohen Anteil von Heranwachsenden mit Migrationshintergrund in Warteschleifen der Berufsvorbereitung, dass die positive Reaktion auf Berlin braucht dich! regelrecht befreiend wirkte. Der kluge Claim »Berlin braucht dich!« wurde gut aufgenommen, sodass mehr Schülerinnen und Schüler aus Einwandererfamilien den Öffentlichen Dienst als eine Option für eine Ausbildung in den Blick nahmen. Der gleich zu Beginn eintretende Erfolg war entscheidend. »Eine Ausbildung im Öffentlichen Dienst kann sich lohnen« war die Botschaft an die Einwanderungsbevölkerung - und auch die Be- 
triebe verstanden, dass sie über Berlin braucht dich! interessante Nachwuchskräfte gewinnen könnten.

W.K.: Rückblickend betrachtet: Hätte integrationspolitisch - also für die Jugendlichen mit Migrationshintergrund - in dieser Aufbruchsperiode »mehr herausgeholt« werden können?

A.G.: Ich habe stets den Modellcharakter der Senatsinitiative Berlin braucht dich! unterstrichen. Ich meine damit, dass man Erfahrungen aus dem interkulturellen Ansatz von Berlin braucht dich! stärker in anderen Ausbildungsprogrammen nutzen sollte. Dabei ist das Erfahrungsspektrum von Berlin braucht dich! breit und schließt interkulturelle Missverständnisse, Diskriminierungen und auch die Überprüfung von Einstellungsvoraussetzungen und Curricula von Ausbildungsgängen ein.

Die Übertragung auf andere Ausbildungsprogramme gelang nur zum Teil. Es gelang in Fällen, in denen die jeweilige politische Leitung eine abteilungs- und ressortübergreifende Zusammenarbeit einforderte. Nach meiner Erfahrung ist ein starker Impuls der Leitung entscheidend, damit eine $\mathrm{Ab}$ stimmung der unterschiedlichen Zielsetzungen gelingen kann: Hier die erfolgreiche Durchführung von Ausbildungen und die interkulturelle Öffnung der Prozesse.

W.K.: Die enge Zusammenarbeit zwischen Schulen und Betrieben in Berlin braucht dich! - Konsortium genannt - wird als eine der Besonderheiten und Erfolgsbedingungen herausgehoben. Zugleich wird aber erkennbar, dass das Konsortium selbst nicht Treiber der Weiterentwicklung ist, sondern eher der Integrationsbeauftragte und der beauftragte Träger BQN. Hätte man das Konsortium stärker machen können? Hat der Konsortialansatz über diesen Fall hinaus Potenzial?

A.G.: Die Zusammenarbeit im Konsortium hat $\mathrm{zu}$ einem erkennbaren Diskurswechsel geführt, der das Potenzial hat, das jahrzehntelange Blaming-ofthe-other zu beenden. Vielfach heißt es aus der Wirtschaft, viele Schülerinnen und Schüler seien nicht ausbildungsreif und daran sei das Bildungssystem schuld; andererseits wird den Betrieben mangelnde Bereitschaft vorgehalten, den Interessen und Anforderungen einer geänderten Schülerschaft zu entsprechen. Das hohe Potenzial im Konsortium sehe ich darin, dass hier die 
Fachleute aus abgebenden Schulen und aufnehmenden Betrieben Übergänge vorbereiten, auf einander abstimmen und damit die wechselseitigen Vorbehalte mindern. Hingegen hatte ich nicht die Erwartung gehabt, dass das Konsortium die Sache stärker in die Hand nimmt und den Prozess antreibt.

W.K.: Hätte eine andere Zusammensetzung des Konsortiums in dieser Hinsicht bessere Ergebnisse erwarten lassen?

A.G.: Dass das Konsortium nicht stärker antreibt, liegt an der Dominanz der Arbeitsebene, die mit ihren jahrelangen Erfahrungen in die Zusammenarbeit geht. Das führt dazu, dass Veränderungen nur in kleinen Schritten erfolgen können. Hingegen zeigen sich Geschäftsleitungen häufig eher bereit, sich für übergreifende Ziele zu öffnen. Es werden durchaus Spannungen zwischen der jeweiligen Arbeits- und Leitungsebene deutlich. Die Leitungen durchgängig einzubeziehen, hat sich generell als unrealistisch erwiesen. Folglich war das gewählte Verfahren pragmatisch richtig: Neben den Abstimmungen auf Arbeitsebene im Konsortium werden einmal im Jahr Gespräche mit den Leitungen geführt.

Das ist allgemein gesagt. Die engere Einbindung der Leitungsebene kann sehr produktiv sein. Eine sehr gute Erfahrung war der Prozess mit der Metall- und Elektroindustrie, den die Leitungen auf Gewerkschaftsseite und des Unternehmensverbandes sehr produktiv antrieben.

W.K.: Die Zusammensetzung des Konsortiums erwies sich dann hinsichtlich der Betriebsbegegnungen als fruchtbar, aber hinsichtlich der tatsächlichen Öffnung der Ausbildung in den beteiligten Betrieben für Schüler*innen der beteiligten Schulen als schwierig?

A.G.: Ich glaube nicht, dass dies an der Zusammensetzung des Konsortiums liegt. Berlin braucht dich! hat sich sehr stark auf den Zugang in eine Ausbildung konzentriert. Damit lag der Schwerpunkt auf Berufsorientierung, und dabei sind die Betriebsbegegnungen ein entscheidendes Instrument.

Änderungen der Prozesse in der Ausbildung wurden auch bearbeitet, vor allem hinsichtlich der Kurrikula. Das wäre zu intensivieren, wobei man sehen wird, wie weitreichend sich die Lehrerinnen und Lehrer daran beteiligen. 
Der erfolgreiche Abschluss von Ausbildungen wird mittlerweile auch in den Blick genommen und zum Beispiel durch Clubs unterstützt, in denen Auszubildende mit jungen Kolleginnen und Kollegen zusammenkommen, die ihre Ausbildung schon abgeschlossen haben (Peer-Ansatz).

W.K.: Die trotz stark verbesserter BO nach wie vor schwachen Übergänge in Ausbildung führen $\mathrm{ab} 2014 \mathrm{zu}$ einer Ernüchterung und Überprüfung der bisherigen Praxis. Eine Erkenntnis war: Benachteiligung ist hartnäckiger als erwartet. Hätte dies nicht früher zum Thema werden können und müssen? Und kann man die Umsetzung gewonnener Einsichten beschleunigen?

A.G.: Es ist sehr gut, dass die Berufsorientierung verbessert werden konnte und dass Berlin braucht dich! zur interkulturellen Öffnung der Berufsorientierung beigetragen hat. Dabei hat der Erfolg von Berlin braucht dich! unterschiedliche Gründe: Wirksam war wohl vor allem die klare Botschaft des Senats, dass man Heranwachsende aus Einwanderer-Communities als zukünftige Kolleg*innen sucht, und die direkte Ansprache in den Communities. Intern haben wir frühzeitig dabei einen so genannten Creaming-Effekt reflektiert; gemeint ist damit, dass wir in dieser Phase vorrangig Schülerinnen und Schüler erreichten, die bereits gut vorbereitet waren, die einen guten mittleren Schulabschluss oder sogar ein Abitur schaffen könnten und also ihren Weg auch ohne besondere Unterstützung finden würden. Ich habe es damals als durchaus positiv bewertet, dass gut vorbereitete Jugendliche den Öffentlichen Dienst als eine Option in den Blick nahmen. Das hat vielen Zukunftschancen eröffnet und den Öffentlichen Dienst bunter gemacht.

Hinzu kommt, dass Berlin braucht dich! jährlich die Einstellungsdaten vorlegte und dokumentierte, wie viele Jugendliche aus Einwandererfamilien ihre Ausbildung im Öffentlichen Dienst und bei Öffentlichen Unternehmen begannen. Diese Zahlen wiesen in der Regel nach oben. Wir hatten also gute Zwischenergebnisse, was kritische Selbstreflexion nicht unbedingt fördert.

Offenkundig geht die stärkere Ausbildungsbeteiligung von Migranten*innen auch auf die Aktivitäten von Berlin braucht dich! zurück: auf die Werbung und die erwähnten Botschaften, die zum Beispiel Ausbildungsverantwortliche im Öffentlichen Dienst und in den Öffentlichen Unternehmen erreichten. Die Erfolge ließen sich jedoch nicht auf einzelne Maßnahmen von Berlin braucht dich! zurückführen. Zwar konnte eine Reihe von Schulen, die mit BQN im Konsortium arbeitete, den Übergang in die duale Ausbil- 
dung markant steigern. Zum Beispiel gab es Integrierte Sekundarschulen in benachteiligten Quartieren, aus denen früher null Prozent der Schulabgänger direkt in die Ausbildung gehen. Wenn solche Schulen im Folgejahr fünf Prozent schaffen, ist das ein Unterschied ums Ganze. Statistisch wird ein solcher Erfolg aber kaum wahrgenommen.

Damit komme ich zu einer Schwierigkeit im Grundansatz von Berlin braucht dich!, die ich - wenngleich aus guten Gründen - in hohem Maß selbst verantworte. Denn ich hatte den Träger gedrängt, in Schulen mit einem besonders hohen Anteil von Heranwachsenden aus Einwandererfamilien tätig zu werden. Das sind in Berlin allerdings Schulen in benachteiligten Quartieren, die von Arbeitslosigkeit und SGB II Bezug geprägt sind. Und das bedeutet, dass viele Schüler*innen aus ihrem familiären Leben wenig Erfahrung aus der Arbeitswelt mitbringen.

Aus diesem Befund heraus wurde die im Buch dargestellte Vierstufigkeit von Berlin braucht dich! als Grundstruktur der Berufsorientierung entwickelt, die sodann in die Landeskonzeption Berufs- und Studienorientierung übernommen wurde. Die Vierstufigkeit ist ein kluges, zielführendes Instrument. Den Aufbau von vier auf einander folgenden Betriebserfahrungen bewerte ich als sehr positiv. Im Rückblick meine ich allerdings, dass wir das Instrument überfordert haben. Die Distanz zur Arbeitswelt ist bei der beschriebenen Zielgruppe wohl zu manifest, als dass sie mit vier befristeten Betriebsbegegnungen (Praktika und Schnupperkursen und einem Bewerbertag) ausgeglichen werden könnte.

Verschiedentlich habe ich diesen Befund als »hartnäckigere Benachteiligung als erwartet« bezeichnet. Das ist wohl zutreffend und darauf ist zu reagieren. Man wird bei Berlin braucht dich! von dem Ziel abgehen müssen, dass alle Schulabgänger"innen den direkten Übergang aus der Schule in die duale Ausbildung schaffen können, und längere Übergangsphasen in den Blick nehmen müssen. Das bedeutet, dass Berlin braucht dich! die anschließende Phase der Berufsvorbereitung mit berücksichtigen sollte, was auf eine Verlängerung der Übergangsphase hinauslaufen würde. Angebote in der Übergangsphase gibt es als Maßnahmen der Berufsvorbereitung bereits seit langem, die allerdings vielfach als Maßnahmen in der Warteschleife diskreditiert werden. Vor dem Hintergrund dieser nicht unbegründeten Kritik sind die angebotenen Maßnahmen auf ihre Effizienz hin und ihre interkulturelle Ausgestaltung hin zu prüfen. 
W.K.: Im Aufmerksamkeitshorizont des Konsortiums war - so scheint es die Orientierung auf die Öffnung der Ausbildung zugunsten der Förderung von Berufsorientierung in den Hintergrund getreten. Hatte sich die ursprüngliche Ausrichtung von Berlin braucht dich! auf die Öffnung der Ausbildung verloren? Oder müssen hier Primär- und Sekundäreffekte von Berlin braucht dich! Unterschieden werden?

A.G.: Es war nicht falsch, so stark auf Berufsorientierung zu setzen, weil damit Nachteile beim Zugang in die Ausbildungen ausgeglichen werden können. Sicherlich sind weitere Hilfen während der Ausbildung nötig. Ansätze hierzu bestehen mit den sogenannten BQN-Klubs, die auf eine Unterstützung durch Peers setzen. Auch die Bearbeitung der Kurrikula von Ausbildungsgängen mit ihrer Prüfung interkultureller Anforderungen kann Ausbildungserfolge unterstützen.

Das Konsortium bietet hierfür herausragende Chancen. Da große Unternehmen - und nicht nur der Öffentliche Dienst - beteiligt sind, kann man ein Modell entwickeln, das beispielgebend für andere Berufszweige wirken wird. Das ist für die Unternehmen - wie für die Jugendlichen - Herausforderung und Chance zugleich.

Die Herausforderung sehe ich darin, dass man - anders als in der Berufsorientierung, bei der die Schulverwaltung als bestimmende Akteurin fungiert - hinsichtlich der Ausbildung eine viel größere Zahl von Verantwortlichen hat. neben Kammern und Innungen auch die Agentur für Arbeit und andere Einrichtungen, die Hilfen anbieten. Die Senatsverwaltung ist hier nur eine beteiligte Akteurin, die m.E. jedoch eine koordinierende Initiative übernehmen sollte mit dem Ziel, einen verbindlichen Rahmen für interkulturell adäquate Ausbildungsgänge zu setzen. Das Konsortium sollte mit den beteiligten Öffentlichen Unternehmen das role model werden.

W.K.: Ab 2015 rückt im engeren Feld des Leitvorhabens Berlin braucht dich! die Erhöhung der Zugänge in Ausbildung aus den beteiligten Schulen bei den beteiligten Betrieben immer mehr ins Zentrum. Ein Pilotprojekt, das für eine Anzahl von motivierten Schüler*innen den Zugang ohne die bisherigen Auswahlverfahren oder weitgehend unabhängig von ihnen ermöglichen soll, entsteht. Eine Reihe von Betrieben macht mit, Vorbehalte aber bleiben. Sind solche Vorbehalte verständlich und nachvollziehbar und wie kann mit ihnen umgegangen werden? 
A.G.: Der Verzicht auf Einstellungstests - oder zumindest ihre geringere Bewertung - in Einstellungsverfahren ist ein spannendes Experiment. Führt der Verzicht auf Tests zu besseren Ergebnissen? Erweisen sich die Tests gar nicht als so zielführende Indikatoren für einen späteren Erfolg in der Ausbildung? Die Evaluation des Pilotvorhabens wird hierzu Erkenntnisse liefern.

Der Pilot wird öffentlich eher positiv wahrgenommen. Die von Dir angesprochenen Vorbehalte haben einen Bezug zu den Hierarchien in Unternehmen. Die Arbeitsebene verteidigt vielfach Verfahren, die weithin als funktionstüchtig angesehen werden, und stellt sich damit gegen die Vorstände, die einer Beteiligung am Piloten zugestimmt haben. Natürlich sind solche Vorbehalte möglichst offen zu bearbeiten. Dabei sollten alle Beteiligten bereit sein, ihre eigenen Annahmen zu revidieren. Natürlich starten die durch den Piloten bevorzugten Jugendlichen mit schwierigen Startchancen. Sie verfügen in der Regel über schlechtere Schulerfahrungen und man wird prüfen müssen, ob solche Nachteile tatsächlich in der Ausbildung ausgeglichen werden können. Dabei darf kein zusätzlicher Erfolgsdruck auf die Auszubildenden ausgeübt werden. Es ist ermutigend, dass einige Ausbildungsleitungen sich mit Verve an dem Experiment beteiligen. Wie gesagt, halte ich eine ergebnisoffene Herangehendweise für entscheidend.

W.K.: Wenn auch aus integrationspolitischer Perspektive auf das Potenzial, das Lernen im Ernstraum von Betrieb und Beruf bietet, nicht verzichtet werden kann: Wie kann erreicht werden, dass mehr Schüler*innen, die bisher abseits gestanden haben, nicht nur in die Ausbildung gelangen, sondern diese auch mit Erfolg durchlaufen? Was kann der Integrationsbeauftragte hierzu beitragen?

A.G.: Berlin braucht dich! erreicht in den vier Stufen der Berufsorientierung eine durchaus relevante Zahl von Schülerinnen und Schülern der Sekundarstufe I. Aber längst nicht alle Heranwachsenden aus Einwandererfamilien, die Unterstützung benötigen, werden erreicht. Das kann man von dem Projekt auch nicht erwarten. Nötig ist eine intensivere Zusammenarbeit mit allen in der Ausbildung verantwortlichen Institutionen. Die Ausweitung von Berlin braucht dich! auf die Öffentlichen Unternehmen, die in den 2010er Jahren erfolgte, war ein ermutigender Erfolg. Die stärkere Zusammenarbeit mit der Berliner Handwerkskammer, die wir 2018 vorbereitet haben, ist ein weiterer aussichtsreicher Schritt. Eine zukunftsträchtige Option für BQN sehe 
ich darin, die interkulturelle Beratung zu den Ausbildungskurrikula, die mit einigen Öffentlichen Unternehmen begonnen wurde, auf andere Branchen auszuweiten.

W.K.: Es sieht so aus, als habe der Eintritt einer großen Zahl junger Geflüchteter wie ein Impuls zur Verbesserung struktureller Bedingungen für Integration und Teilhabe auch im Feld von Schule und Beruf geführt. Kann man nach den 2o10er Jahren jetzt erneut von einem Aufbruch sprechen? Und welche Rolle spielt dabei die Integrationspolitik?

A.G.: Auf einen hohen Handlungsdruck wurde positiv reagiert; das ist das Entscheidende. Der Merkel'sche Satz »wir schaffen das!«, der mittlerweile ja häufig ironisiert wird, wurde vor Ort ernst genommen. Eine positive Grundstimmung, die durch zivilgesellschaftliche Gruppen maßgeblich gestärkt wurde, haben auch die Verwaltungen aufgegriffen. Hilfen für Geflüchtete wurden als zusätzliche Herausforderung akzeptiert, und dafür hat der Senat den Bezirken zusätzliche Stellen zugewiesen, so dass sich die Zahl der Mitarbeiter*innen für Integrationsmaßnahmen erfreulich stark erhöhte. Zudem hat der Senat die Mittel für Beratungsangebote verstärkt, zum Beispiel durch Integrationslotsinnen und -lotsen, die die Verständigung zwischen Geflüchteten und Mitarbeiter*innen in den Ämtern unterstützen.

Konkrete Beispiele in Bezug auf die Ausbildung von Geflüchteten sind Praktika in unterschiedlichen Handwerksberufen sowie Berufsorientierung in so genannten Willkommensklassen an Berufsschulen. In diesen Klassen werden Geflüchtete unterrichtet, die schon älter sind und daher nicht in die Regelschule kommen.

Die Integrationspolitik hat dabei eine begleitende Funktion, die der Senat insofern unterstrichen hat, als er mir als Integrationsbeauftragtem die Koordination der Maßnahmen zur Integration und Partizipation von Geflüchteten zugeteilt hat. Meine Nachfolgerin setzt das fort.

W.K.: Integrations-, besser Teilhabepolitik in der Einwanderungsstadt ist eine Querschnittsaufgabe, die sich impulsgebend und kooperativ auf alle Handlungsfelder beziehen müsste - eine Mammutaufgabe. Vor diesem Hintergrund und rückblickend: War und ist eine herausgehobene Schwerpunktsetzung auf die Öffnung der Ausbildung vertretbar? Oder ist diese Schwerpunktsetzung eher exemplarisch zu verstehen? Dann aber: Wie können die 
dortigen Erfahrungen und Einsichten für andere Felder nutzbar gemacht werden?

A.G.: Grundsätzlich bearbeitet die/der Integrationsbeauftragte natürlich alle Handlungsfelder des Senats. Ein jüngeres Beispiel ist das schon erwähnte Gesamtkonzept zur Integration und Partizipation Geflüchteter von Dezember 2018, an dem die Verwaltungen für Soziales, Jugend, Bildung, Arbeit, Stadtentwicklung und Gesundheit, aber auch Inneres besonders engagiert mitgearbeitet haben. Beim Aktionsplan zur Einbeziehung ausländischer Roma - um einen weiteren Querschnittsansatz zu nennen, den in Berlin der Integrationsbeauftragte koordiniert - ist ebenfalls ein breites Spektrum von Senats- und Bezirksverwaltungen beteiligt.

In der Regel führen die Verwaltungen ihre Programme selbst federführend durch und beziehen den Integrationsbeauftragten jeweils ein. Die Fachverwaltungen sind aufgrund des Partizipations- und Integrationsgesetzes dazu verpflichtet, zum Teil beziehen sie aus eigenem Interesse den Beauftragten ein. Denn klar ist ja, dass sich in Berlin alle Programme für benachteiligte Quartiere oder Zielgruppen in hohem Maß an die Einwanderungsbevölkerung richten.

Ich habe ein besonders starkes Augenmerk auf den Übergang in die berufliche Ausbildung gelegt, da damit je individuell die Weichen für einen späteren beruflichen Erfolg gestellt werden. Aus diesem Grund habe ich den Übergang ins Arbeitsleben auch als integrationspolitisches Zukunftsthema besonders wichtig wahrgenommen und mit Berlin braucht dich! ein eigenes Programm durchgeführt. Das ist natürlich exemplarisch aufzufassen - angesichts der Funktion des Integrationsbeauftragten, der Bedeutung des Handlungsfeldes und der großen Zahl darin aktiver Akteure.

Insgesamt ist der exemplarische Ansatz aufgegangen. Die Integrationsabteilung hat aufgrund eigener Erfahrungen im Handlungsfeld fachliche Kompetenz zum Übergangsmanagement erworben; viele Partner haben die interkulturellen Ansätze in der Berufsorientierung und zum Übergangsmanagement insgesamt positiv aufgenommen, namentlich die Bundesagentur für Arbeit und die Bildungsverwaltung. Allerdings entstanden auch Konkurrenzen, die im Spannungsfeld zwischen Fachpolitik und dem integrationspolitischen Querschnittsansatz angesiedelt sind. Nicht in allen Fällen ließen sich diese Konkurrenzen konstruktiv auflösen, obgleich die interkulturelle 
Öffnung der Fachprogramme in Berlin gesetzlich gefordert (PartIntG) und auch Ziel der Regierungspolitik des jetzigen und der vorherigen Senate ist.

W.K.: Es spricht einiges dafür, dass die Frage nach der Ausgestaltung eines Berliner Übergangssystems Schule-Arbeitswelt nun noch einmal aussichtsreicher auf die Tagesordnung kommen könnte. Wie wird dies integrationspolitisch gesehen? Wie könnte diese Querschnittsaufgabe platziert werden?

A.G.: Die Erfahrungen, die wir in diesem Buch reflektiert haben, drängen auf ein kohärentes Übergangssystem, in dem durchgängig ein Fokus auf Migration und Teilhabe als Querschnittsaufgabe zu etablieren ist. Ich kann mir vorstellen, dass Deine Annahme zutrifft. Dafür sprechen vor allem drei Gründe: Erstens der weiterhin bestehende Erfolgsdruck, heranwachsende Geflüchtete zum Ausbildungserfolg zu führen; zweitens das gewachsene Verständnis dafür, wie wichtig es ist, allen Heranwachsenden aus Einwandererfamilien Zukunftschancen zu öffnen und dass das eine wichtige $\mathrm{Zu}$ kunftsfrage für unsere Gesellschaft ist; drittens und vor allem aber, dass viele Verantwortliche in den Unternehmen mittlerweile die Erfahrung machen, dass Erfolge hierbei möglich sind - auch mit Auszubildenden, die nach üblichen Kriterien nicht als bestens vorbereitet wirken.

Den vierten Punkt, nämlich die demografische Entwicklung und die daraus resultierende Nachfrage nach Auszubildenden, der häufig zuerst genannt wird, bewerte ich eher als einen unterstützenden Kofaktor. Am wichtigsten erscheinen mir positive Erfahrungen zu sein, die Zugangswege geöffnet haben, und die auf andere Branchen übertragen werden können.

W.K.: Abschließend: Den Zeitraum ab 2007 bis heute und darin noch einmal insbesondere die Öffnung der Berufsausbildung betrachtend: Kann man integrations- und teilhabepolitisch zufrieden sein? Was ist gelungen? Was bleibt zu tun? Worin liegen die zentralen Herausforderungen der nahen $\mathrm{Zu}$ kunft?

A.G.: Zur interkulturellen Öffnung der Übergänge in den Beruf konnten wir seit 2006 in Berlin gute Erfolge erzielen. Am wichtigsten ist wohl das generelle Signal, dass Erfolge möglich sind, die sich für die Stadt insgesamt positiv auswirken und vielen jungen Menschen aus Einwandererfamilien Zukunftschancen eröffnen. 
Allerdings hat sich gezeigt, dass die interkulturelle Öffnung von Ausbildungsgängen kein Selbstläufer ist. Hier sehe ich zwei Hauptprobleme, die ich zu Beginn des Vorhabens unterschätzt hatte: erstens die in meiner vorletzten Antwort angesprochene Konkurrenz. Zweitens hatte ich erwartet, dass für die Prozesse in einzelnen Branchen und Handlungsfeldern ein vorübergehender interkultureller Input ausreichen würde, der dann durch die jeweils verantwortlichen Akteure fortgeführt würde. Ich hatte angenommen, dass man im engeren Sinn exemplarisch vorgehen könnte und die interkulturelle Öffnung - sozusagen als zeitgemäße Reform und Modernisierung - Branche für Branche, Handlungsfeld für Handlungsfeld durcharbeiten könnte. Das hätte vorausgesetzt, dass der Integrationsbeauftragte sich jeweils nach einer initiierenden Phase aus den Prozessen herausziehen könnte, um sich anderen Feldern zuzuwenden. Das gelang in den durch Berlin braucht dich! entwickelten Konsortien nicht, da die beteiligten Akteure die koordinierende Funktion des Trägers BQN für unverzichtbar halten. Diese Einschätzung wird man zukünftig erneut bewerten müssen.

Auf einer allgemeineren Ebene ist der Erfolg unstrittig. Berlin braucht dich! hat zur interkulturellen Öffnung von Ausbildungsgängen beigetragen, in den Öffentlichen Unternehmen die Einstellung von Auszubildenden aus Einwandererfamilien verstärkt und dabei die Bereitschaft unterstützt, auch jungen Menschen mit schwierigeren Startchancen Zukunftschancen zu eröffnen. Hierüber besteht weitgehend Einvernehmen in der Stadt, und daher wurde der Ansatz auch von den drei Senaten, die seit Beginn von Berlin braucht dich! im Amt waren, durchgängig unterstützt. Sehr viele Verantwortliche, die sich zuvor nicht mit der interkulturellen Öffnung befasst hatten, haben sich mittlerweile auf den Weg gemacht und kooperieren mit dem/der Integrationsbeauftragten. 



\section{Abkürzungsverzeichnis}

Bbd!: $\quad$ Berlin braucht dich!

BO: $\quad$ Berufsorientierung

BQN: $\quad$ Berufliches Qualifizierungsnetzwerk für Migrantinnen und Migranten

BSO-Teams: Berufs- und Studienorientierung-Teams

BVBO: $\quad$ Berliner Vertiefte Berufsorientierung

DGB: $\quad$ Deutscher Gewerkschaftsbund

IGM: Industriegewerkschaft Metall

ISS: Integrierte Sekundarschule

M\&E: $\quad$ Metall- und Elektroindustrie

MSA: $\quad$ Mittlerer Schulabschluss

ÖD: $\quad$ Öffentlicher Dienst

OSZ: Oberstufenzentrum

PartIntG: Gesetz zur Regelung von Partizipation und Integration in Berlin (28.12.2010)

SGB II: Sozialgesetzbuch II

SVR: $\quad$ Sachverständigenrat deutscher Stiftungen für Integration und Migration

WAT: Wirtschaft Arbeit Technik (Unterrichtsfach) 



\section{Literaturverzeichnis}

Arbeitsgemeinschaft Weinheimer Initiative: Hoyerswerdaer Erklärung Lehrstück Übergang. Jahresforum 2011, in: Arbeitsgemeinschaft Weinheimer Initiative (Hg.): Lokale Bildungsverantwortung, Stuttgart 2013. Aumüller, Jutta: Vielfalt in Pankow. Die Beteiligung von MigrantInnen auf lokaler Ebene, Berlin 2014.

BDA: Berufsschule der Zukunft, Berlin 2012.

Beicht, Ursula/Granato, Mona 2011: Prekäre Übergänge vermeiden - Potenziale nutzen. Junge Frauen und Männer mit Migrationshintergrund an der Schwelle von der Schule zur Ausbildung (=WISO Diskurs), Bonn 2011, S. 11ff.

Beikler, Sabine/Fiedler, Maria: Jetzt arbeitet die neue Behörde für Flüchtlinge, in: Tagesspiegel vom 1.8.2016

Beikler, Sabine: „Wir sind in der Realität schon viel weiter«. Berlins Integrationsbeauftragter Andreas Germershausen im Interview über Rassismus, Heimatgefühle und Debatten, die Populisten helfen. In: Tagesspiegel vom 30.7.2018.

Benalia, Emina: Neues Willkommenszentrum in Berlin nimmt seine Arbeit auf, in: Berliner Morgenpost vom 18.08.2016.

Berufsbildung aktuell: »Rekrutierungs-Pipeline in die Schulen verlegen. BBaktuell im Interview mit Arno Hager«, in: Berufsbildung aktuell 2/2014, S. 3.

Bochum, Ulrich/Butler, Jeffrey/Kohlmeyer, Klaus/Odenwald, Stephanie: Soziale Spaltungen in Berlin, Hamburg 2016, insbesondere der Abschnitt: »Soziale Spaltung durch ungleiche Bildungschancen - ungleiche Bildungschancen durch soziale Spaltung«, S. 87-114.

BQN Berlin (Hg.): Dokumentation Konsortialtagung 2015. https://www.bqnberlin.de/site/assets/files/1144/doku_konsortialtreffen_2015.pdf 
Brock, Ditmar/Hantsche, Brigitte/Kühnlein, Gertrud/Meulemann, Heiner/ Schober, Karen (Hg.): Übergänge in den Beruf. Zwischenbilanz zum Forschungsstand, München 1991; vgl. hierzu auch Kruse, Wilfried/Expertengruppe: Jugend. Von der Schule in die Arbeitswelt, Stuttgart 2010. Büchter, Karin: Berufsschulen in der dualen Ausbildung und regionalen Wirtschaft. Gleichberechtigte Partnerschaft durch Reformen (=Working Paper Forschungsförderung 059), Düsseldorf 2018.

Bundesministerium für Bildung und Forschung: Modelle und Strategien zur Verbesserung der Bildungsbeteiligung von Jugendlichen mit Migrationshintergrund. Ergebnisse der Initiativstelle Berufliche Qualifizierung von Migrantinnen und Migranten (IBQM) beim Bundesinstitut für Berufsbildung (BIBB), Berlin 2006.

Buschkowsky, Heinz: Neukölln ist überall, Berlin 2012.

Çinar, Safter: »Das Partizipations- und Integrationsgesetz für Berlin«.

Czerwenka, Kurt: Umgang mit schwierigen Schülern. Erkenntnisse aus der Verhaltenspsychologie Vortrag vor der Konferenz Starke Schule am 4. Mai 2009 in Berlin, zusammengefasst in: Bildungsportal NRW des Ministeriums für Schule und Weiterbildung des Landes Nordrhein-Westfalen unter der Überschrift »eder siebte Schüler gilt als schwierig«www. schulministerium.nrw.de, aufgerufen am 17.1.2016

Dahms, Nathaly: Ausbildung und Weiterbildung als Teil der interkulturellen Personalentwicklung (=Berliner Hefte zur interkulturellen Verwaltungspraxis), Berlin 2012; oder auch: Pampel, Jana/Raschke, Christian: Die Interkulturelle Öffnung eines arbeitsmarktpolitischen Programms - am Beispiel des Landesprogramms Mentoring, Berlin 2016.

Der Beauftragte des Berliner Senats für Integration und Migration (Hg.): Alle mit dabei? Potenziale der Berufsausbildung für Jugendliche aus Einwandererfamilien, Berlin 2019.

Der Beauftragte des Berliner Senats für Integration und Migration: Berliner Senat investiert 42 Millionen in die Integrationspolitik, Pressemitteilung vom 21.6.2018.

Der Beauftragte des Senats von Berlin für Integration und Migration (Hg): Vielfalt fördern - Zusammenhalt stärken. Das Berliner Integrationskonzept, Berlin 2007. Der Senat hat das Integrationskonzept am 3.7.2007 beschlossen, Drucksache 16/0715. 
Der Beauftragte des Senats von Berlin für Integration und Migration: Indikatoren zur Messung von Integrationserfolgen (=Berliner Beiträge zur Integration und Migration), Berlin 2007.

Der Beauftragte des Senats von Berlin für Integration und Migration: Expertise »Kompetenzerfassung - FrauenComputerZentrumBerlin (=Berliner Beiträge zur Integration und Migration), Berlin 2006.

Der Beauftragte des Senats von Berlin für Integration und Migration: Integration und Migration - Ein Wegweiser für Berlin. Ausgabe 2014, Berlin 2014.

Der Regierende Bürgermeister. Senatskanzlei: Pressemitteilung vom 21.6.2006.

Dobischat, Rolf/Kühnlein, Gertrud/Schurgatz, Robert: Ausbildungsreife. Ein umstrittener Begriff beim Übergang jugendlicher in die Berufsausbildung (=Arbeitspapiere der Hans-Böckler-Stiftung 189), Düsseldorf 2012.

Enggruber, Ruth/Rützel, Josef: Berufsausbildung junger Menschen mit Migrationshintergrund. Eine repräsentative Befragung von Betrieben, Gütersloh 2014.

Famulla, Gerd-Ewald/Butz, Bert/Deeken, Sven/Michaelis, Ute/Möhle, Volker/Schäfer, Birgit: Berufsorientierung als Prozess. Persönlichkeit fördern, Schule entwickeln, Übergang sichern. Ergebnisse aus dem Programm >Schule-Wirtschaft/Arbeitsleben<, Hohengehren 2008.

Foroutan, Naika: »Die postmigrantische Gesellschaft«: www.bpb.de/gesellschaft/migration/kurzdossiers/205295 am 21.4.2015.

Friedrich-Ebert-Stiftung: Kommunalpolitik verstehen im Land Berlin. Für ein junges Politikverständnis, Berlin 2010.

Geißler, Rainer: »Migration und Integration«, www.bpb.de/izpb/198020/migration-und-integration vom 16.12.2014.

Germershausen, Andreas/Narr, Wolf-Dieter: Flucht und Asyl in der Politik der Bundesrepublik, Berlin 1988, S. 23-34.

Gesemann, Frank: »Berlin: »Einwanderungsstadt >under construction«? Von der Beauftragtenpolitik zur strategischen Steuerung«, in: Frank Gesemann/Roland Roth (Hg.): Lokale Integrationspolitik in der Einwanderungsgesellschaft, Wiesbaden 2009.

Goethe, Katharina: Erfolg in der Ausbildung. Projektergebnisse und Empfehlungen für eine bessere Ausbildungssicherung bei Auszubildenden mit Migrationshintergrund, Berlin 2012. 
Greinert, Wolf-Dietrich/Wolf, Stefan: Die Berufsschule. Radikale Neuorientierung oder Abstieg zur Restschule? Frankfurt a.M. 2010.

Großkurth, Heike/Lex, Tilly/Lichtwardt, Nina/Müller, Sylvia/Tillmann, Frank (2015): Prekäre Übergangsverläufe. Entstehungsbedingungen risikobehafteter Übergänge, Landeshauptstadt München, März 2015.

Gyapay, Borbála: »Die Veränderung des ethnischen Bildes Berlins«, in: Zeitschrift für amtliche Statistik Berlin Brandenburg 3/2012.

Hasselmann, Jörn/Frenzel, Veronica/Kneist, Sigrid/Van Bebber, Werner: »Räumung am Oranienplatz. Kein Friede den Hütten«, in: Der Tagesspiegel vom 8.4.2014.

Häußermann, Hartmut/Kapphan, Andreas: Berlin - von der geteilten zur gespaltenen Stadt? Sozialräumlicher Wandel seit 1990, Wiesbaden 2002.

Häußermann, Hartmut: »Wohnen und Quartier: Ursachen sozialräumlicher Segregation«, in: Ernst-Ulrich Huster, Jürgen Boeckh, Hildegard MoggeGrothjan (Hg.): Handbuch Armut und soziale Ausgrenzung, Wiesbaden 2008.

Helbig, Marcel/Jähnen, Stefanie: Wie brüchig ist die soziale Architektur unserer Städte? Trends und Analysen der Segregation in 74 deutschen Städten (=Discussion Paper P 2018-001), Berlin 2018.

Hoesch, Kirsten: Migration und Integration. Eine Einführung, Wiesbaden 2018.

Holl, Hans-Peter: >Gute< Lehrer an berufsbildenden Schulen aus der Schülerperspektive«, in: Die berufsbildende Schule 60/2008.

Holtappels, Heinz Günter: »Schulentwicklung unter herausfordernden Bedingungen«, Auftaktveranstaltung mit den Projektschulen am 20.03.2015 in Essen, Universität Duisburg-Essen http://schulen-staerken. de/wp-content/uploads/2014/o9/Auftaktveranstaltung-Holtappels.pdf

Hyams ,Judith: „Öffentlicher Dienst. Endlich angekommen. Busfahrer, Polizist, Krankenpfleger: Berlin braucht Azubis - und wirbt gezielt um Jugendliche mit Migrationshintergrund«, in: Der Tagesspiegel vom 18.4.2016.

IG Metall Berlin: Beschluss Boo2: Gelingende Übergänge von der Schule in die Arbeitswelt: Zukunftssicherung für die Metropole Berlin. In: DGB Berlin-Brandenburg: Beschlüsse der 7. DGB Bezirkskonferenz BerlinBrandenburg 2018.

JugendtheaterBüro Berlin der Initiative Grenzenlos e.V.: Berufsorientierung durch Jugendkulturarbeit. Einfluss der Arbeit im JugendtheaterBüro 
Berlin auf die Lebensperspektiven teilnehmender Jugendlicher 20092012, Berlin 2012, www.grenzen-los.eu/wp-content/uploads/2012/05/ Brosch \%C3\%BCre_JTB_Web.pdf

Kalman, Michael/Metje, Ute M./Rofles, Manfred/Kohlmeyer, Klaus: »Evaluation von Integrationspolitik. Bericht über die Frühjahrstagung von DeGEval mit einem Beitrag des damaligen Integrationsbeauftragten Piening «, https://www.researchgate.net/publication/297832061_Evaluation_von_Integrationspolitik_Fruhjahrstagung_de

Köhler, Regina: »In Berliner Berufsschulen fällt der Unterricht zu oft aus«, in: Berliner Morgenpost vom 16.11.2015.

Kohlmeyer, Klaus/Kruse, Wilfried/Zschiesche, Tilman: Betriebspraktika als Baustein der Berliner Berufsorientierung und Berufsvorbereitung, unveröffentlichte Manuskripte, Berlin 2013.

Kohlmeyer, Klaus/Pavkovic, Gari: »Fokus Migration und die kommunale Steuerung am Übergang Schule und Beruf «, in: Arbeitsgemeinschaft Weinheimer Initiative (Hg.): Lokale Bildungsverantwortung, Stuttgart 2013.

Kohlmeyer, Klaus: Gutachten. Berlin: Europäische Modellmetropole für Integration und Vielfalt. Die schulische und berufliche Bildung junger Migrant/innen, Berlin 2006.

Kruse, Wilfried/Mütherich, Birgit: Arbeitsmarktintegration von Migrantinnen und Migranten in Dortmund - Eine Handlungshilfe (=sfs Beiträge aus der Forschung), Dortmund 2000.

Kruse, Wilfried/Strauß, Jürgen/Braun, Frank/Müller, Matthias: Rahmenbedingungen der Weiterentwicklung des Dualen Systems beruflicher Bildung (=Arbeitspapiere Hans-Böckler-Stiftung 167), Düsseldorf 2009.

Kruse, Wilfried: Nachhaltigkeitsreport. Lokale Netzwerke zur Berufsintegration von jungen Migrantinnen und Migranten am Ende der Projektförderung(=sfs-Beiträge aus der Forschung Band 158), Dortmund 2007.

Kruse, Wilfried: Berlin braucht dich! - über den Öffentlichen Dienst hinaus? Eine Recherche zu Integration und dualer Berufsausbildung. Expertise für BQN Berlin/Dortmund 2010.

Kruse, Wilfried: "Lernort Betrieb im >Dualen Systemః: Spannungsreiche Verhältnisse«, in: Kuda, Eva/Strauß, Jürgen/Spöttl, Georg/Kaßebaum, Bernd (Hg.): Akademisierung der Arbeitswelt? Zur Zukunft der beruflichen Bildung, Hamburg 2012. 
Kruse, Wilfried: »Dimensionen landesweiter Steuerung am Übergang Schule - Beruf«. Vortrag anlässlich der Abschlussveranstaltung des BMBFProjektes RÜM Berlin am 15.03.2012 im Palais Kulturbrauerei, Berlin www.perspektive-berufsabschluss.de > downloads und: vimeo.com > XENOS Panorama Berlin > Videos.

Kruse, Wilfried: »Wiederentdeckt. Der Betrieb als Lernort mit Potential«, https://www.berlin-braucht dich.de/fileadmin/user_upload/PDFs/Wiederentdeckt_Lernort_Betrieb.pdf, 2015.

Künzel, Manfred »Ein Netz von Entwicklungsräumen. Wege zu einer lebensweltorientierten Schule«, in: Thomas, Peter Martin/Calmbach, Marc (Hg.): Jugendliche Lebenswelten. Perspektiven für Politik, Pädagogik und Gesellschaft, Heidelberg 2013.

Lehmkuhl, Kirsten/Eckelt, Marcus/Schöler Cornelia: Ausbildung - eine attraktive Zukunftsoption? Schülerinnen und Schüler mit Migrationshintergrund erleben die Arbeitswelt. Expertise für BQN Berlin, Berlin 2011.

Lindemann, Hans-Jürgen (Hg): Lern- und Arbeitsaufgaben sowie Projektund Arbeitsaufgaben in der Lernfeldarbeit, Berlin 2013.

Lippegaus-Grünau, Petra: »Individualisierung in der betrieblichen Berufsausbildung. Entwicklungslinien und neue Ausbildungsmodelle«, in : Severing, Eckart/Weiß, Reinhold (Hg.): Individuelle Förderung in heterogenen Gruppen in der Berufsausbildung. Befunde - Konzepte - Forschungsbedarf, Bielefeld 2014.

Michler, Inga: Arbeitgeber bemängeln Qualität der Berufsschulen, in: Die Welt vom 22.2.2015.

Neuhof, Ursula/Yazar, Serdar/Gębala, Marta: Werkseinstellungen auf Vielfalt setzen (=Impulse zu Vielfalt 2018/2), Berlin 2018.

Nowakowski, Gerd: Berliner Schulpolitik. Das Ergebnis der Bildungspolitik ist verheerend, in: Tagespiegel vom 25.6.2018.

Paul-Kohlhoff, Angela: »Berufliche Bildung in Deutschland - ein männliches Projekt?«, in: Kuda, Eva/Strauß, Jürgen/Spöttl, Georg/Kaßebaum, Bernd (Hg.): Akademisierung der Arbeitswelt? Zur Zukunft der beruflichen Bildung, Hamburg 2012.

Piening, Dorothea/Rauner, Felix: Umgang mit Heterogenität. Eine Handreichung des Projekts KOMET. Universität Bremen 2010.

Rauner, Felix/Piening, Dorothea: Umgang mit Heterogenität in der beruflichen Bildung. Eine Handreichung des Projekts KOMET, Bremen 2010. 
Regionaldirektion Berlin-Brandenburg der Bundeagentur für Arbeit: Strategische Neuorientierung der Berufsorientierung, Dokument vom 2. Dezember 2008.

Sachverständigenrat deutscher Stiftungen für Integration und Migration: Papiertiger oder Meilensteine? Die Integrationsgesetze der Bundesländer im Vergleich (=Policy Brief Oktober 2017), Berlin 2017.

Sachverständigenrat deutscher Stiftungen für Integration und Migration: Segregation an deutschen Schulen: Ausmaß, Folgen und Handlungsempfehlungen für bessere Bildungschancen, Berlin 2013.

Sachverständigenrat deutscher Stiftungen für Integration und Migration: Einwanderungsgesellschaft 2010. Jahresgutachten 2010 mit Integrationsbarometer, Berlin 2010.

Sarrazin, Thilo: Deutschland schafft sich ab. Wie wir unser Land aufs Spiel setzen, München 2010.

Scherr, Albert (Hg.): Diskriminierung migrantischer Jugendlicher in der beruflichen Bildung. Stand der Forschung, Kontroversen, Forschungsbedarf, Weinheim 2015.

Scherr, Albert/Janz, Caroline/Müller, Stefan: „Einleitung: Ausmaß, Formen und Ursachen der Diskriminierung migrantischer Bewerber/innen um Ausbildungsplätze«, in: Albert Scherr (Hg.): Diskriminierung migrantischer Jugendlicher in der beruflichen Bildung. Stand der Forschung, Kontroversen, Forschungsbedarf, Weinheim 2015.

Schulte, Axel: »Integrationspolitik in der Bundesrepublik Deutschland «, in: Der moderne Staat - Zeitschrift für Public Policy 1/2011, S. 29-60, hier S. 38. Im Folgenden wird weiter auf diesen Aufsatz Bezug genommen.

Schultheis, Kathrin/Sell, Stefan: »Die drei Sektoren der beruflichen Bildung

- Übergangssystem«, https://www.bpb.de/politik/innenpolitik/arbeitsmarktpolitik/187852/uebergangssystem vom 11.8.2014.

Senatorin für Arbeit, Integration und Frauen 2012: BerlinArbeit. Strategische Neuausrichtung der Arbeitsmarkt- und Berufsbildungspolitik des Landes Berlin in der Legislaturperiode 2011 bis 2016, Eckpunkte, Berlin/BerlinArbeit. Gemeinsames Rahmen-Arbeitsmarktprogramm des Landes Berlin und der Regionaldirektion Berlin-Brandenburg der Bundesagentur für Arbeit, veröffentlicht am 30.7.2013. https://www.berlintransfer.net/de/wissenspool/politischer-rahmen/534-berlin-arbeit 
Severing, Eckart/Weiß, Reinhold (Hg.): Individuelle Förderung in heterogenen Gruppen in der Berufsausbildung. Befunde - Konzepte - Forschungsbedarf, Bielefeld 2014.

Siebel, Walter: Die Kultur der Stadt, Berlin 2015.

Tillmann, Klaus-Jürgen 2013: »Die Bewältigung von Übergängen im Lebenslauf - eine biografische Perspektive«, in: Bellenberg, Gabriele/Forell, Matthias (Hg.): Bildungsübergänge gestalten. Ein Dialog zwischen Wissenschaft und Praxis, Münster 2013.

Vieth-Entus, Susanne: Die Mischung stimmt nicht, in: Tagesspiegel vom 3.6.2015.

Weiss, Karin/Alan, Birsan: »Integrationsgesetze in den Bundesländern«, in: Karl-Heinz Meier-Braun/Reinhold Weber (Hg.), Deutschland Einwanderungsland. Begriffe - Fakten - Kontroversen, Stuttgart 2017.

Wensierski, Hans-Jürgen/Lübcke, Claudia: »Als Moslem fühlt man sich hier auch zu Hause«. Biographien und Alltagskulturen junger Muslime in Deutschland, Opladen/Berlin/Toronto 2012.

Wiechert-Beyerhaus, Ralf auf dem JahresforumExtra im Dezember 2016: www.kommunalekoordinierung.de/files/Jahresforen/Jahresforum_Extra_2016_Berlin/Dokumentation/Forum2_Ralf_Wiechert-Beyerhaus_ Folien.pdf

Zawatka-Gerlach, Ulrich: 15 Prozent offene Stellen. In der Berliner Verwaltung fehlt massiv+ Personal, in: Tagespiegel vom 4.1.2018. 


\section{Pädagogik}

Kay Biesel,

Felix Brandhorst,

Regina Rätz,

Hans-Ullrich Krause

Deutschland

schützt

seine Kinder!

Eine Streitschrift zum Kinderschutz

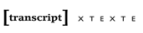
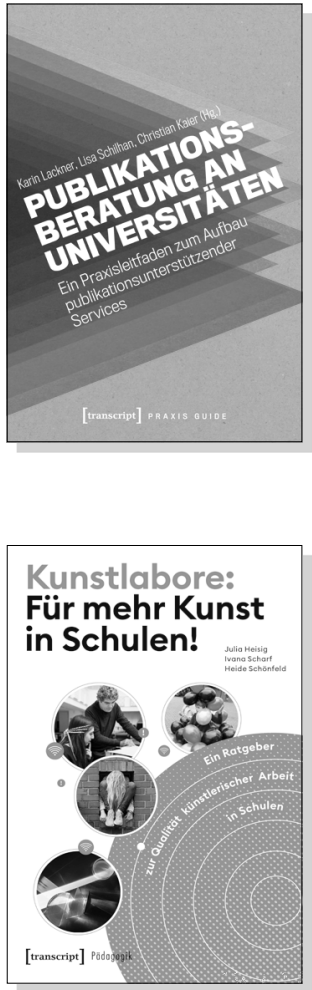

Kay Biesel, Felix Brandhorst, Regina Rätz, Hans-Ullrich Krause Deutschland schützt seine Kinder!

Eine Streitschrift zum Kinderschutz

2019, 242 S., kart., 1 SW-Abbildung

22,99€ (DE), 978-3-8376-4248-3

E-Book: 20,99 € (DE), ISBN 978-3-8394-4248-7

EPUB: $20,99 €$ (DE), ISBN 978-3-7328-4248-3
Karin Lackner, Lisa Schilhan, Christian Kaier (Hg.)

\section{Publikationsberatung an Universitäten}

Ein Praxisleitfaden zum Aufbau

publikationsunterstützender Services

2020, 396 S., kart., 14 SW-Abbildungen

39,00€ (DE), 978-3-8376-5072-3

E-Book: kostenlos erhältlich als Open-Access-Publikation PDF: ISBN 978-3-8394-5072-7

ISBN 978-3-7328-5072-3

Julia Heisig, Ivana Scharf, Heide Schönfeld

\section{Kunstlabore: Für mehr Kunst in Schulen!}

Ein Ratgeber zur Qualität künstlerischer Arbeit in Schulen

2020, 216 S., Klappbroschur, durchgängig vierfarbig 27,99€ (DE), 978-3-8376-4985-7

E-Book: kostenlos erhältlich als Open-Access-Publikation

PDF: ISBN 978-3-8394-4985-1 


\section{Pädagogik}

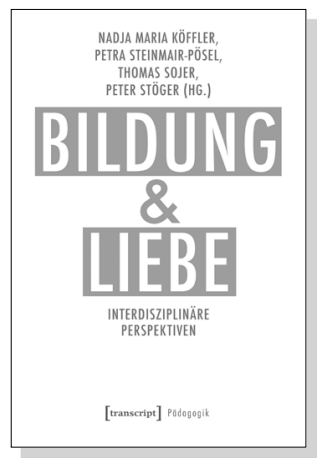

Nadja Köffler, Petra Steinmair-Pösel,

Thomas Sojer, Peter Stöger (Hg.)

Bildung und Liebe

Interdisziplinäre Perspektiven

2018, 412 S., kart., 11 SW-Abbildungen

39,99€ (DE), 978-3-8376-4359-6

E-Book:

PDF: 39,99 € (DE), ISBN 978-3-8394-4359-0

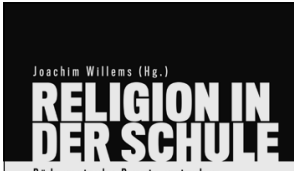

Joachim Willems (Hg.)

\section{Religion in der Schule}

Pädagogische Praxis

zwischen Diskriminierung und Anerkennung

2020, 432 S., kart.

39,00€ (DE), 978-3-8376-5355-7

E-Book: kostenlos erhältlich als Open-Access-Publikation

PDF: ISBN 978-3-8394-5355-1

[transcript] pitıbik

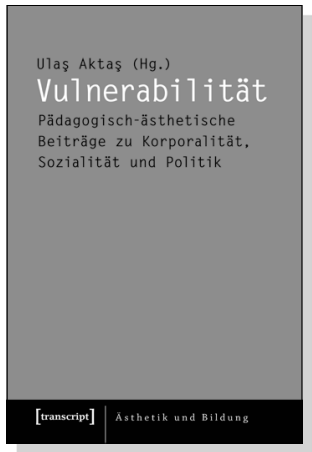

Ulaș Aktaș (Hg.)

\section{Vulnerabilität}

Pädagogisch-ästhetische Beiträge

zu Korporalität, Sozialität und Politik

2020, 194 S., kart., 26 SW-Abbildungen, 26 Farbabbildungen 39,00€ (DE), 978-3-8376-5444-8

E-Book:

PDF: $38,99 €(D E)$, ISBN 978-3-8394-5444-2 

